University of Louisville

ThinkIR: The University of Louisville's Institutional Repository

$5-2020$

\title{
Experimental and numerical study of process parameters effects towards part quality and porosity during powder bed additive manufacturing processes.
}

Subin Shrestha

University of Louisville

Follow this and additional works at: https://ir.library.louisville.edu/etd

Part of the Manufacturing Commons

\section{Recommended Citation}

Shrestha, Subin, "Experimental and numerical study of process parameters effects towards part quality and porosity during powder bed additive manufacturing processes." (2020). Electronic Theses and Dissertations. Paper 3580.

https://doi.org/10.18297/etd/3580

This Doctoral Dissertation is brought to you for free and open access by ThinkIR: The University of Louisville's Institutional Repository. It has been accepted for inclusion in Electronic Theses and Dissertations by an authorized administrator of ThinkIR: The University of Louisville's Institutional Repository. This title appears here courtesy of the author, who has retained all other copyrights. For more information, please contact thinkir@louisville.edu. 


\title{
EXPERIMENTAL AND NUMERICAL STUDY OF PROCESS PARAMETERS EFFECTS TOWARDS PART QUALITY AND POROSITY DURING POWDER BED ADDITIVE MANUFACTURING PROCESSES
}

\author{
By
}

Subin Shrestha

\author{
A Dissertation \\ Submitted to the Faculty of the \\ J.B. Speed School of Engineering of the University of Louisville \\ in Partial Fulfillment of the Requirements \\ for the Degree of \\ Doctor of Philosophy \\ in Mechanical Engineering \\ Department of Mechanical Engineering \\ University of Louisville \\ Louisville, Kentucky, United States
}

August 2020 
Copyright 2020 by Subin Shrestha

All rights reserved 

EXPERIMENTAL AND NUMERICAL STUDY OF PROCESS PARAMETERS EFFECTS TOWARDS PART QUALITY AND POROSITY DURING POWDER BED ADDITIVE MANUFACTURING PROCESSES

By

Subin Shrestha

A Dissertation Approved on

August 03, 2020

By the following Dissertation Committee:

Dissertation Direction: Dr. Kevin Chou

Dr. Thomas L. Starr

Dr. Thomas Berfield

Dr. Hui Wang 


\section{DEDICATION}

This dissertation is dedicated to my parents

Mr. Ishwor Man Shrestha

And

Mrs. Chandra Keshari Shrestha

For their love, sacrifice, and guidance throughout my life 


\section{ACKNOWLEDGEMENTS}

First and foremost, I would like to express my sincerest gratitude to my adviser, Dr. Kevin Chou, for his continuous support throughout my Ph.D. His guidance and motivation helped me in all time of the research. I feel honored to have him as my Ph.D. adviser and mentor.

Besides my adviser, I would like to thank other committee members, Dr. Thomas Starr, Dr. Thomas Berfield, and Dr. Hui Wang for their advice and insightful comments.

I am sincerely grateful to NASA (No. NNX11AM11A), NIST (70NANB16H029), and NSF (1662662 and 1921263) for their financial support during my Ph.D. study.

I also want to acknowledge my fellow lab mates: Dr. Bo Cheng, Dr. Xiaoqing Wand, Tahmina Keya, Dr. Shanshan Zhang, Santosh Rauniyar, and Aref Vali, for the stimulating discussions and all the fun we have had in the last five years. I like to extend my gratitude to Dr. Bo Cheng, who helped me during my first year of research.

I would like to thank parents, Ishwor Man Shrestha and Chandra Keshari Shrestha for their love and support. I am grateful to my late sister, Sabina Shrestha, to my brother, Sumit Shrestha, and to my dear wife, Sudha Rana Magar for supporting me throughout difficult times and encouraging me to achieve my goals. 


\begin{abstract}
EXPERIMENTAL AND NUMERICAL STUDY OF PROCESS PARAMETERS EFFECTS TOWARDS PART QUALITY AND POROSITY DURING POWDER BED ADDITIVE MANUFACTURING PROCESS
\end{abstract}

Subin Shrestha

August 03, 2020

Powder bed additive manufacturing (PB-AM) process utilizes an electron beam or a laser as a heat source to melt the metallic powder particles. These processes have the capability of freeform fabrication, however certain defects such as porosity, high surface roughness, etc. would hinder its application. It is important to understand the effect of the process parameters and the underlying physical phenomena, which lead to the formation of such defects. In this regard, a three-dimensional (3D) thermo-fluid model is developed to study the effect of beam speed on the surface morphology during powder bed electron beam additive fabrication (PB-EBAF). Besides, the surfaces of PB-EBAF fabricated Ti6Al-4V parts are analyzed using a white-light interferometer. The results show that in general, the build surface roughness along the beam moving direction slightly increases with the scanning speed. On the other hand, the hatch spacing noticeably affects the 
surface roughness in the transverse direction. In addition, the numerical model was modified to incorporate powder particles and study the effect of powder distribution towards the single-track formation during the laser powder bed fusion (LPBF) process. The numerical results show that the single-track morphology and density depend on the process parameters: scanning speed and laser power. Besides, micro-computed tomography (micro-CT) is utilized to characterize the pores formed during the LPBF process. Single tracks were fabricated with linear energy density (LED) ranging from 0.1 $\mathrm{J} / \mathrm{mm}$ to $0.98 \mathrm{~J} / \mathrm{mm}$, and the samples were then scanned using micro-CT to measure keyhole porosity. The results show that the severity of the keyhole porosity increases with the increase of the LED. By keeping the LED constant in another single-track scanning experiment, different combinations of the power and the speed were tested to investigate the individual effect. The results show that for the same LED, the pore number and volume increased with increasing the power to a certain critical level, beyond which, the further increase and power resulted in fewer pore number and lower pore volume.

The experimental results suggested that the dynamic phenomenon of a melt pool during the LPBF process is complex and sensitive to process parameters. Hence, a discrete element method (DEM) is utilized to obtain a powder distribution, which is then used to perform a thermo-fluid simulation using FLOW-3D software. The numerical results indicated that for a constant LED, the keyhole size increases with the increase in the laser power. The keyhole becomes stable at a higher power, which may reduce the occurrence of pores during laser scanning. In addition, a back and forth raster scanning is performed to form three tracks to investigate the effect of scan length on the melt pool 
size at different locations along the laser travel direction. Three scan speeds $(375 \mathrm{~mm} / \mathrm{s}$, $750 \mathrm{~mm} / \mathrm{s}$, and $1500 \mathrm{~mm} / \mathrm{s}$ ) are used with laser power of $195 \mathrm{~W}$, and three tracks are fabricated with $0.5 \mathrm{~mm}, 1 \mathrm{~mm}$, and $1.5 \mathrm{~mm}$ scan lengths. Besides, hatch spacings of 80 $\mu \mathrm{m}$ and $120 \mu \mathrm{m}$ are used. The fabricated samples are analyzed using white light interferometer and metallography. Moreover, a powder scale numerical model is developed to understand the residual heat effect on the melt pool. The results show that the region where the laser changes the direction is the most affected zone where a significant increase in the melt pool size is observed. The depth of the melt pool increased with increasing track number. The delay in the successive laser scan needed to minimize the residual heat effect is calculated for different process parameters. 


\section{TABLE OF CONTENTS}

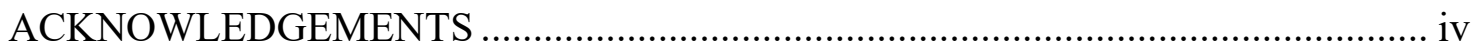

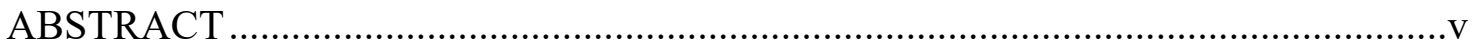

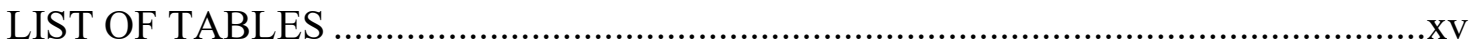

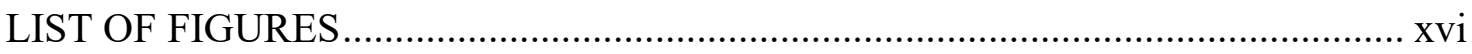

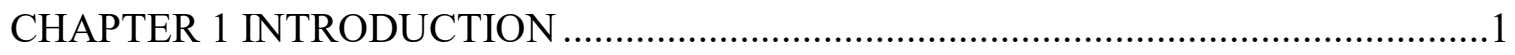

$1.1 \quad$ Background and Motivation .........................................................................

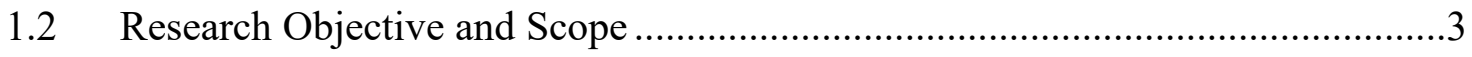

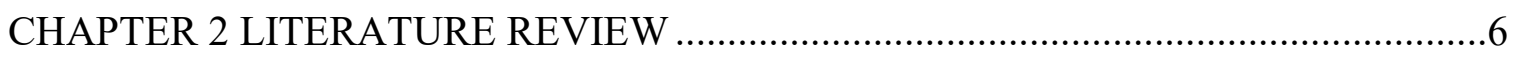

2.1 Thermo-fluid modeling of PB-EBAM process. .....................................................6

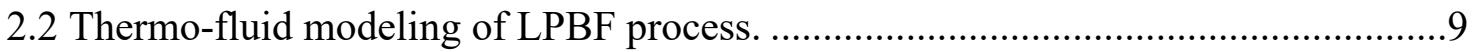

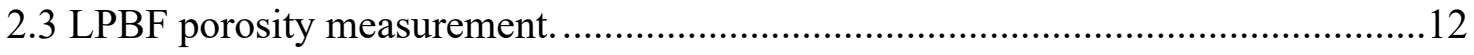

CHAPTER 3 A BUILD SURFACE STUDY OF POWDER-BED ELECTRON BEAM ADDITIVE MANUFACTURING BY 3D THERMO-FLUID SIMULATION AND WHITE-LIGHT INTERFEROMETRY ……………….......................................15

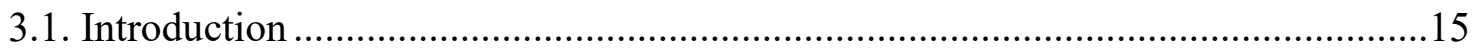


3.2.1 3D model configurations ............................................................................20

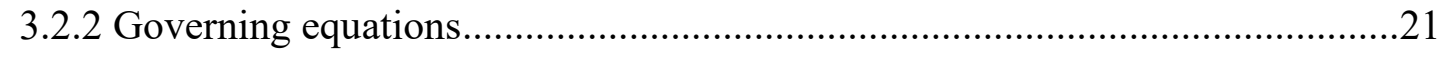

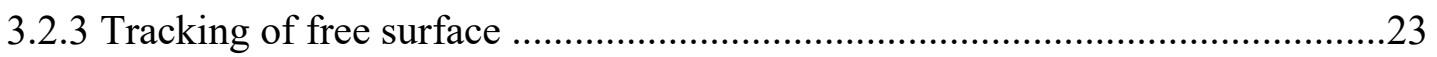

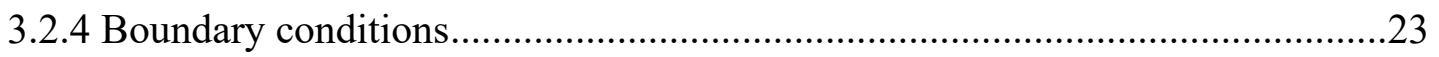

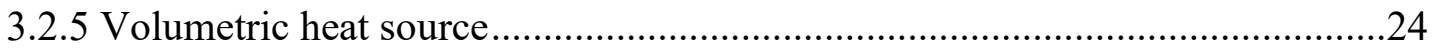

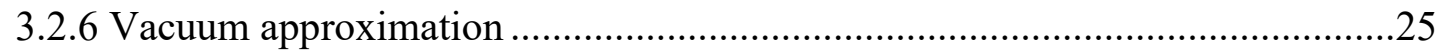

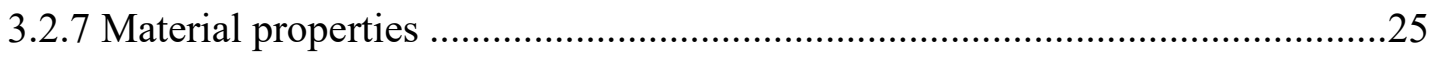

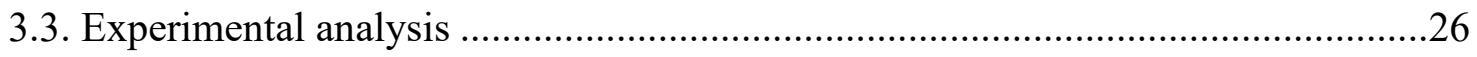

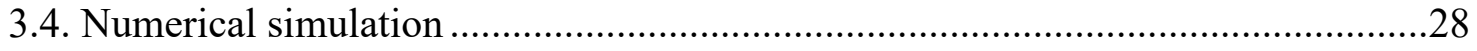

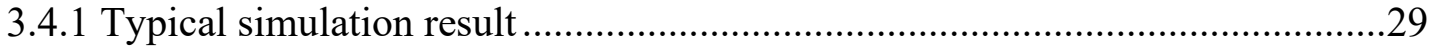

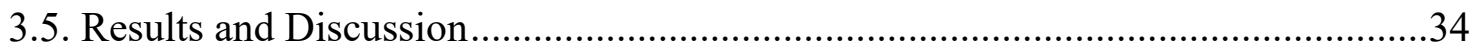

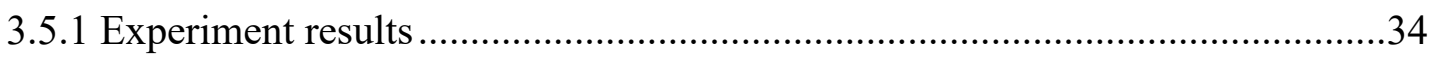

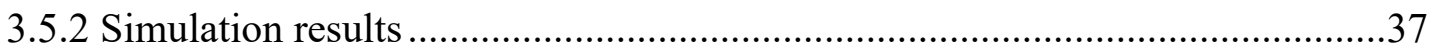

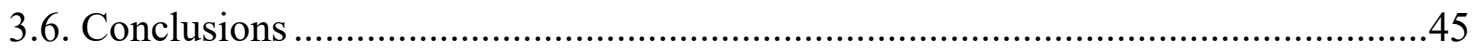

CHAPTER 4 THERMO-FLUID MODELING OF SELECTIVE LASER MELTING: SINGLE-TRACK FORMATION INCORPORATING METALLIC POWDER ..............47

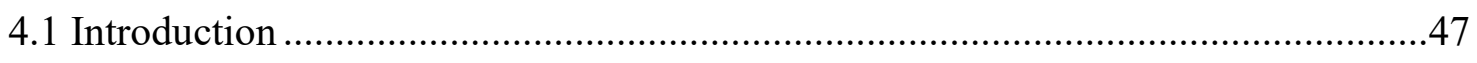

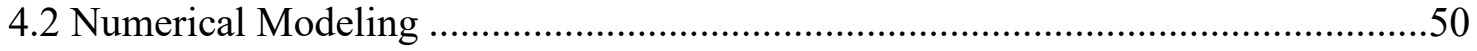

4.2.1 Powder scale model..................................................................................50 
4.2.2 Governing equations

4.2.3 Tracking of free surface

4.2.4 Boundary conditions.

4.2.5 Application of volumetric heat source

4.2.6 Material Properties

4.3 Results and Discussion

4.3.1 Single layer simulation

4.3.2 Two-layer simulation

4.4 Conclusions

CHAPTER 5 A STUDY OF KEYHOLE POROSITY IN SELECTIVE LASER MELTING: SINGLE TRACK SCANNING WITH MICRO-CT ANALYSIS .66

5.1 Introduction .66

5.2 Experimental Approach. .69

5.2.1 SLM specimen design and fabrications .69

5.2.2 Process parameter design .71

5.2.3 Pore measurement and analysis .73

5.3 Results and discussion .75

5.3.1 Typical scanning images .75

5.3.2 Pore characteristics .76

5.3.3 Process effects .79 
5.4 Conclusions

CHAPTER 6 A NUMERICAL STUDY ON THE KEYHOLE FORMATION DURING

LASER POWDER BED FUSION PROCESS .......................................................95

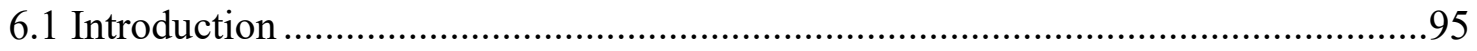

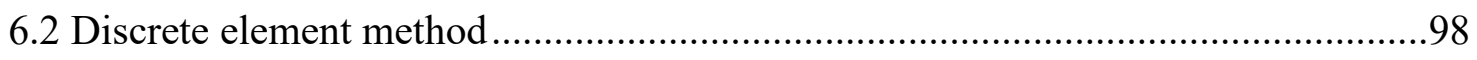

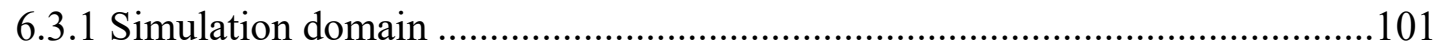

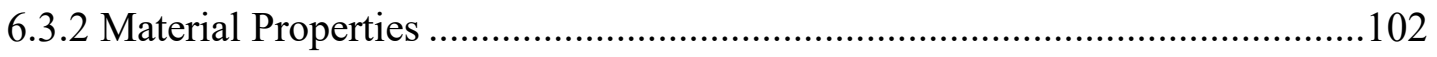

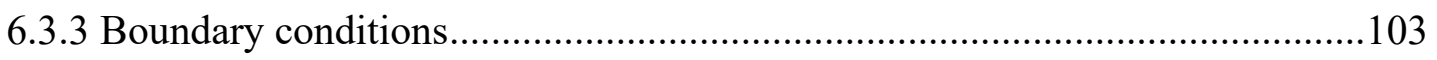

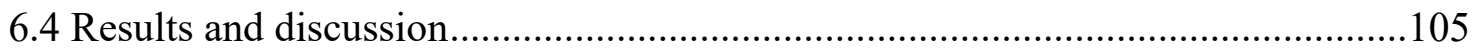

6.4.1 Typical simulation result ...........................................................................105

6.4.2 Effect of power and speed on keyhole formation........................................114

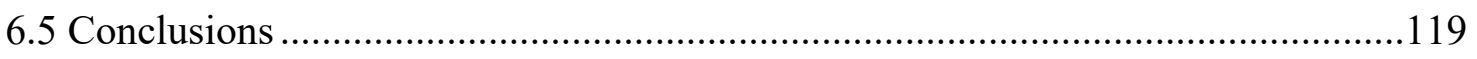

CHAPTER 7 A STUDY OF PORE FORMATION DURING SINGLE LAYER AND MULTIPLE LAYER BUILD BY SELECTIVE LASER MELTING..............................121

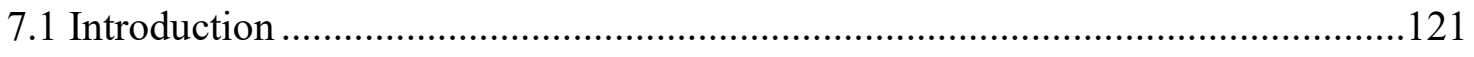

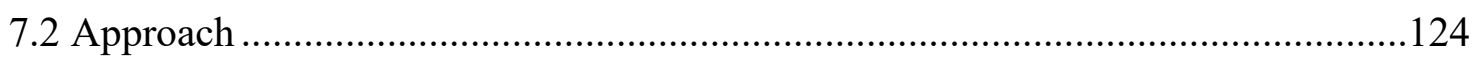

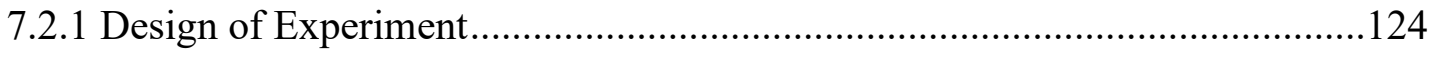

7.2.2 Sample Design and Fabrication......................................................................125

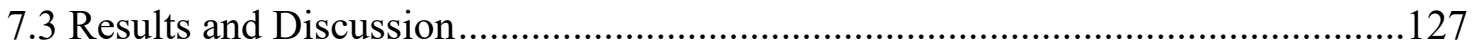

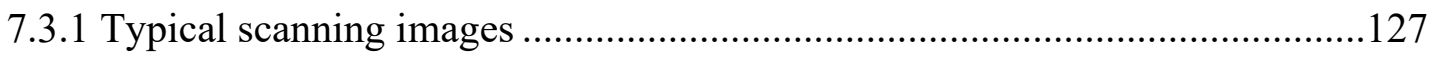


7.3.2 Single layer experiment results

7.3.3 Multiple layer experiment results 130

7.4 Conclusions .134

CHAPTER 8 AN INVESTIGATION INTO MELTING MODES IN SELECTIVE LASER MELTING OF INCONEL 625 POWDER: SINGLE TRACK GEOMETRY

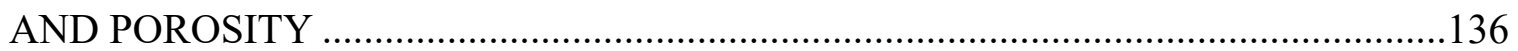

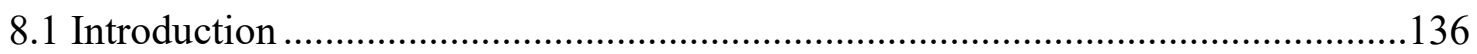

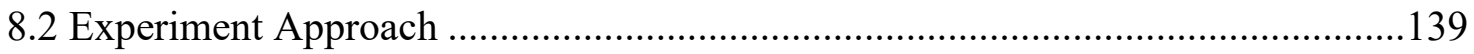

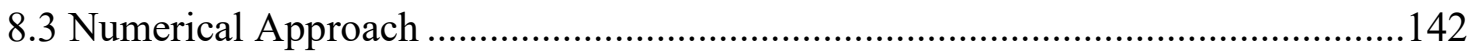

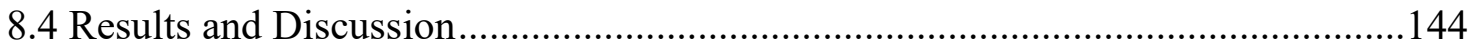

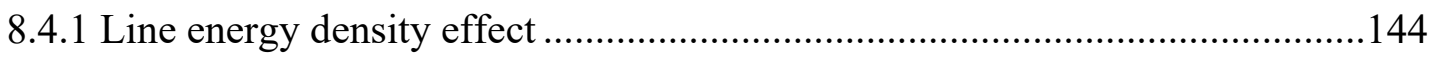

8.4.2 Effect of power and speed at the same energy density .....................................151

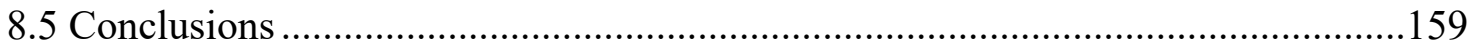

CHAPTER 9 A STUDY OF TRANSIENT AND STEADY-STATE REGIONS FROM SINGLE-TRACK DEPOSITION IN LASER POWDER BED FUSION …………..........161

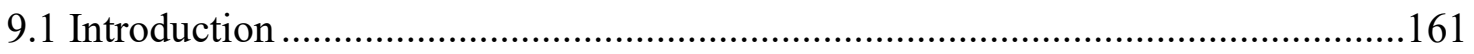

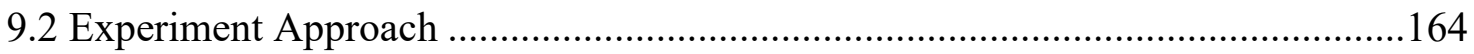

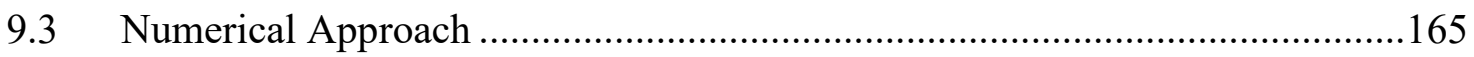

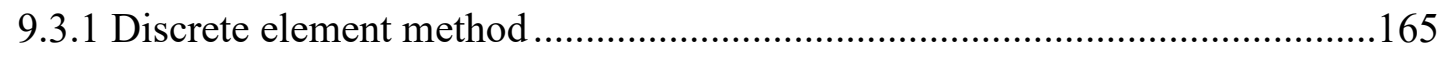

9.3.2 Powder scale thermo-fluid model................................................................168 
9.4 Results and Discussion

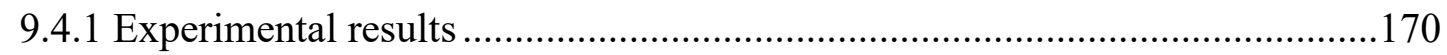

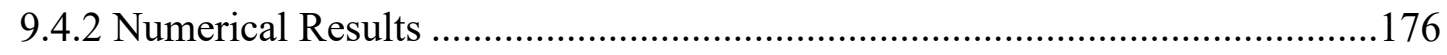

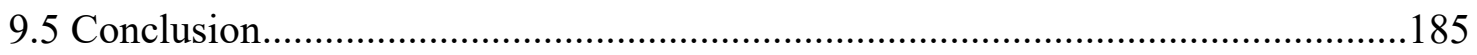

CHAPTER 10 RESIDUAL HEAT EFFECT ON THE MELT POOL GEOMETRY DURING THE LASER POWDER BED FUSION PROCESS ………….......................187

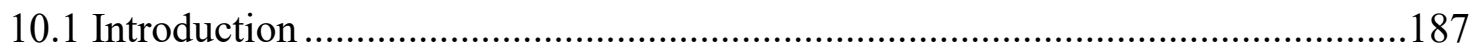

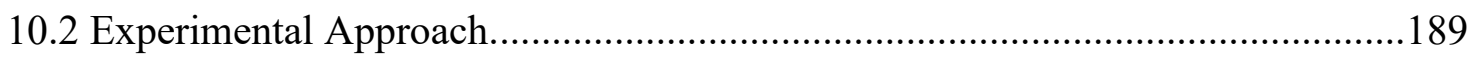

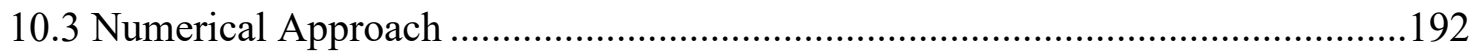

10.3.1 Discrete Element Method ...........................................................................192

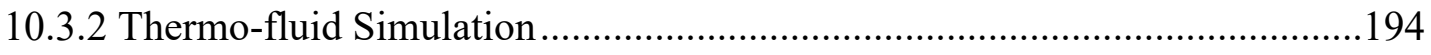

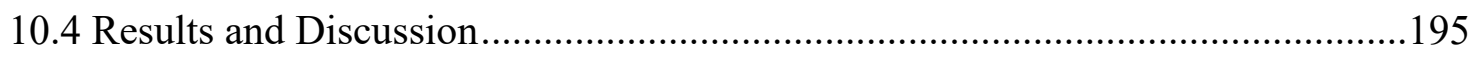

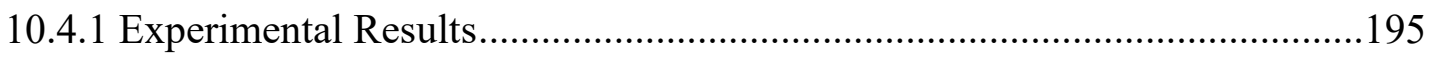

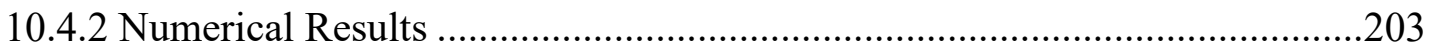

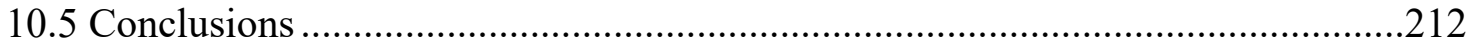

CHAPTER 11 BUILD LOCATION EFFECT ON THE SINGLE-TRACK PORE CHARACTERISTICS FORMED WITH SELECTIVE LASER MELTING PROCESS214

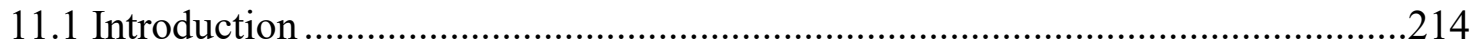

11.2 Experiment Design and Sample Fabrication ..................................................215

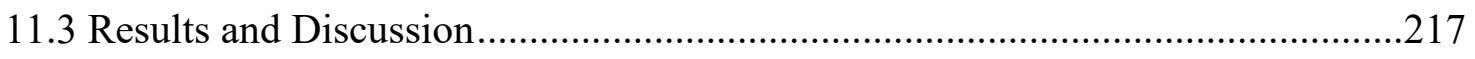


11.4 Conclusions

CHAPTER 12 CONCLUSIONS AND FUTURE WORK.

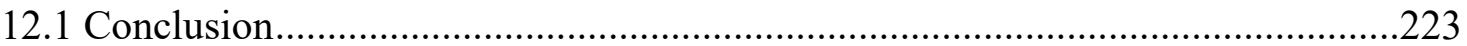

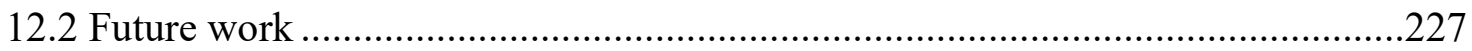

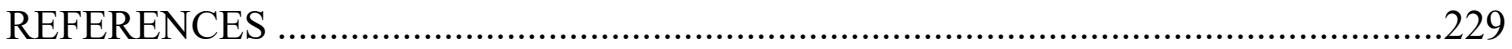

APPENDIX A BUILD LOCATION EFFECT ON THE SINGLE-TRACK PORE CHARACTERISTICS FORMED WITH SELECTIVE LAESR MELTING PROCESS239

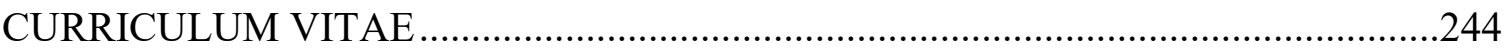




\section{LIST OF TABLES}

Table 3.1 Properties of Ti-6Al-4V and process parameters used in model ...................... 26

Table 4.1 Material properties of Ti-6Al-4V ………………...................................... 56

Table 5.1 Process parameters used to investigate energy density $(\mathrm{J} / \mathrm{mm})$ effect............. 72

Table 5.2 Process parameters used to investigate power and speed effect...................... 73

Table 5.3 Location and properties of 9 pores from $195 \mathrm{~W}$ and $400 \mathrm{~mm} / \mathrm{s}$ parameters..... 78

Table 6.1 Ti-6Al-4V properties for DEM simulation................................................... 99

Table 6.2 Properties of Ti-6Al-4V in a thermo-fluid simulation.................................... 103

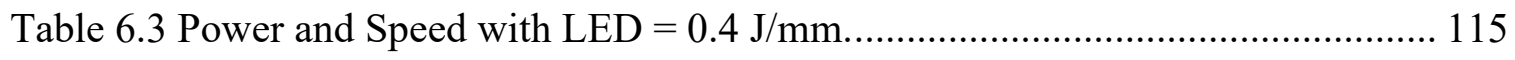

Table 7.1 Process parameters used in single layer and multilayer design..................... 125

Table 8.1 Process parameters used to investigate energy LED (J/mm) effect................ 140

Table 8.2 Process parameters selected from the constant energy density of $0.48 \mathrm{~J} / \mathrm{mm} .141$

Table 8.3 Process parameters selected from the constant energy density of $0.75 \mathrm{~J} / \mathrm{mm} .141$

Table 8.4 Properties of In625 in a thermo-fluid simulation........................................... 144

Table 9.1 . Inconel 625 properties for DEM simulation............................................. 167

Table 9.2 Properties of In625 used in a thermo-fluid simulation. ................................. 170

Table 10.1 Experimental design used to fabricate multi-track samples......................... 190

Table 10.2 Properties of In625 in a thermo-fluid simulation........................................ 195 


\section{LIST OF FIGURES}

Figure 1.1 Schematic drawing of selective laser melting and electron beam melting

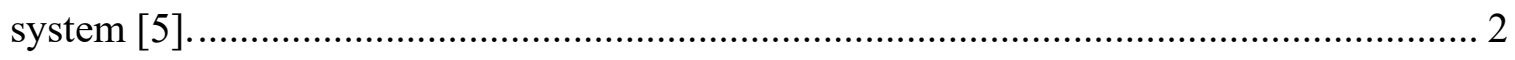

Figure 2.1 Gaussian laser heat source used for LPBF simulations.............................. 10

Figure 3.1 (a) Melting of powder immediately after beam strikes powder layer and (b)

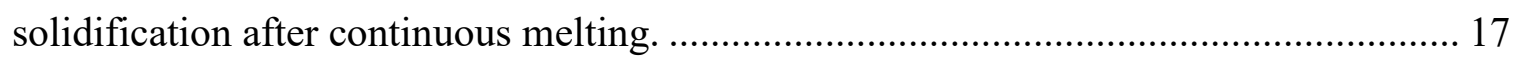

Figure 3.2(a) 3D model used for build surface analysis and detailed view for volume fraction information and (b) raster scanning applied at the top of powder layer............. 21

Figure 3.3 Temperature dependent density, specific heat and thermal conductivity of Ti-

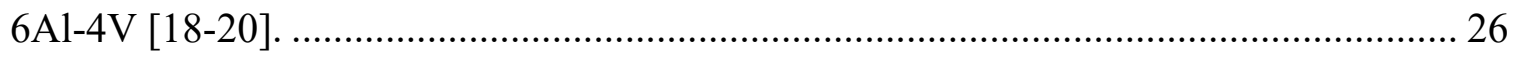

Figure 3.4 Contour plot as displayed by white light interferometer ............................ 28

Figure 3.5 Typical 2D profile output from white light interferometer along (a) scanning

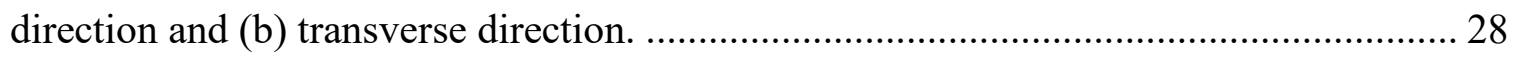

Figure 3.6 (a) Temperature distribution at the end of first scan track, (b) hump formation after solidification in transverse direction and (c) surface profile along scanning direction.

Figure 3.7 Temperature profile along (a) scanning direction and (b) transverse direction

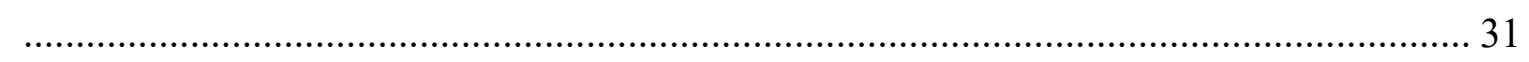

Figure 3.8 Velocity profile within melt pool ......................................................... 31

Figure 3.9 Surface morphology predicted for SF36 case. ...................................... 32 
Figure 3.10 (a) Mixing of powder and solid particles after melting and (b) melt separation. 33

Figure 3.11 Surface height profile along (a) scanning direction and (b) transverse direction. 34

Figure 3.12 Stereoscopic image and isometric view of surface scanned taken from white light interferometer for (a) SF20, (b) SF36, (c) SF50 and (d) SF65 cases. 35

Figure 3.13 Average surface roughness for different SF cases. 36

Figure 3.14 Melt pool shapes from free surface for different SF cases .......................... 38

Figure 3.15 Velocity profile in the transverse direction for different SF cases............... 39

Figure 3.16 Maximum velocity in vertical direction for different SF cases ................... 40

Figure 3.17 Surface morphologies of solidified top surface by simulation for (a) SF20, (b)SF50, (c) SF65, (d)SF65 with $\mathrm{H}=0.3 \mathrm{~mm}$ and (e) SF65 with $\mathrm{H}=0.1 \mathrm{~mm}$. 43

Figure 3.18 Surface roughness from simulation results along the transverse direction for different cases: (a) SF20, (b) SF50, (c) SF65, (d) SF65 with H=0.1 mm and (e) SF65 with $\mathrm{H}=0.3 \mathrm{~mm}$ 44

Figure 4.1 Incoming sphere rolling down over the previously deposited sphere. 51 Figure 4.2 Computational domain with powder particles distribution used for simulation 52

Figure 4.3 2D representation of topmost free surface identification to apply a volumetric heat source 54

Figure 4.4 Temperature dependent material properties of Inconel Ti-6Al-4V [13]. 55

Figure 4.5 Top view of the powder bed showing (a) the melt pool at $t=400 \mu$ s and (b) solidified track at $\mathrm{t}=1600 \mu \mathrm{s}$ along with the temperature distribution $\left({ }^{\circ} \mathrm{C}\right)$ 57 
Figure 4.6 Melt pool dynamics during melting and solidification................................ 58

Figure 4.7 Melt pool comparison between different process parameters ....................... 59

Figure 4.8 Surface morphology for four velocity values at a constant power of 195 W.. 60

Figure 4.9 Melt pool width comparison between experiment and simulation................. 61

Figure 4.10 Solidified single tracks at different scanning speeds............................. 61

Figure 4.11 Image slice of the 3D domain at the shallow region for four scanning speed62

Figure 4.12 Second layer powder addition demonstration.

Figure 4.13 (a) Second layer of powder being defined over first layer and (b) second layer of powder after fully defined.

Figure 4.14 (a) Solidified single track after second layer scanning, (b) Transverse and (c) longitudinal cross section of solidified two layer-single track $(\mathrm{v}=1000 \mathrm{~mm} / \mathrm{s})$. 64

Figure 5.1 (a) CAD model of the specimen (unit in $\mathrm{mm}$ ) and (b) Representation of single track designed on top of previously deposited semi-cylinder. 70 Figure 5.2 (a) Samples after removal from the machine and (b) after support removal... 70 Figure 5.3 (a) Schematic of $\mu \mathrm{CT}$ components and (b) Specimen setup in the CT system 74 Figure 5.4 Selection of region of interest for porosity measurement. 75 Figure 5.5 (a) Coronal (X-Z), (b) Transaxial (X-Y), (C) Sagittal (Z-Y) cross-sectional views and (d) 3D partial cut-off view. 76 Figure 5.6 A 3D pore on right is rendered from the $2 \mathrm{D}$ images from the left. 77 Figure 5.7 (a) Pore location definition and (b) detail view of pores formed at the end of scan track from $195 \mathrm{~W} 400 \mathrm{~mm} / \mathrm{s}$ parameters. 78

Figure 5.8 Single track morphology from different energy densities. 81

Figure 5.9 3D pore view observed at different process parameters. 83 
Figure 5.10 (a) Pore diameter and (b) pore depth summary from different LEDs. 84

Figure 5.11 Total pore volume corresponding to different energy densities used........... 85

Figure 5.12 Average pore volume at different power and speed................................ 86

Figure 5.13 Single track morphology at different process parameters for $\mathrm{LED}=0.48 \mathrm{~J} / \mathrm{mm}$. 88

Figure 5.14 Longitudinal sections from the single tracks with $\mathrm{ED}=0.48 \mathrm{~J} / \mathrm{mm}$. 89

Figure 5.15 Pore diameter and pore depth summary from process parameters of $\mathrm{LED}=0.48 \mathrm{~J} / \mathrm{mm}$. 90

Figure 5.16 Pore count and pore volume obtained at different process parameters from different energy densities (a) $0.32 \mathrm{~J} / \mathrm{mm}$, (b) $0.4 \mathrm{~J} / \mathrm{mm}$ and (c) $0.48 \mathrm{~J} / \mathrm{mm}$. 91

Figure 5.17 Stability of keyhole formation affected the evaporation pressure and the pressure due to at surface tension at different energy densities [91]. 93

Figure 6.1 (a) Powder added to the dispenser platform and (b) powder particles settled over build plate after recoating process. 100

Figure 6.2 3D computational domain used for single track simulation....................... 102

Figure 6.3 Temperature dependent material properties of ti-6al-4v [18] .................... 102

Figure 6.4 Powder and substrate melting during laser application............................ 106

Figure 6.5 Melt region formed after complete melting and solidification..................... 107

Figure 6.6 Melt pool boundary compared with the experiment [103] ........................ 107

Figure 6.7 Equilibrium points during the formation of vapor column [91] ................. 109

Figure 6.8 Multiple reflection vectors from the keyhole wall. 110

Figure 6.9 (a) Velocity field, keyhole profile and breakage of keyhole to form bubble, and (b) 2D temperature and velocity field along longitudinal section. 
Figure 6.10 Fluid flow in transverse direction during keyhole melting. 112

Figure 6.11 Melt pool boundary compared with the experiment for 195w laser power and $400 \mathrm{~mm} / \mathrm{s}$ scan speed [88]. 112

Figure 6.12 Melt region formed after complete melting and solidification. 113

Figure $6.132 \mathrm{D}$ images of the pores formed at the beginning of the single track and their $3 \mathrm{D}$ rendered morphology. 113

Figure 6.14 Pore number and volume from a different level of power with LED $=0.4$ $\mathrm{J} / \mathrm{mm}[97]$. 114

Figure 6.15 Keyhole shape at different time steps from different parameters, (a) $\mathrm{P}=100$ $\mathrm{W}, \mathrm{v}=250 \mathrm{~mm} / \mathrm{s}$, (b) $\mathrm{P}=200 \mathrm{~W}, \mathrm{v}=500 \mathrm{~mm} / \mathrm{s}$, (c) $\mathrm{P}=300 \mathrm{~W}, \mathrm{v}=750 \mathrm{~mm} / \mathrm{s}$ and (d) $\mathrm{P}$ $=400 \mathrm{~W}, \mathrm{v}=1000 \mathrm{~mm} / \mathrm{s}$. 116

Figure 6.16 Intensity dependence in the relationship between vapor column and recoil pressure [20]. 117 Figure 6.17 Temperature distribution when laser has moved $0.7 \mathrm{~mm}$ with $\mathrm{p}=100 \mathrm{w}, \mathrm{v}=$ $250 \mathrm{~mm} / \mathrm{s}$ and $\mathrm{p}=200 \mathrm{w}$ and $\mathrm{v}=500 \mathrm{~mm} / \mathrm{s}$. 118 Figure 6.18 Longitudinal melt region obtained with different power and speed at same energy density. 119

Figure 7.1 Single track geometry and hatch spacing definition in single layer scanning. 125

Figure 7.2 (a) Sample design dimensions and (b) Single layer and multi-layer samples fabricated by EOS M270. 126

Figure 7.3 (a) single layer and (b) multi-layer design along with the scan strategies. .. 126 
Figure 7.4 (a) Coronal (X-Z), (b) Transaxial (X-Y), (c) Sagittal (Z-Y) cross-sectional views and (d) partial cut-off view of a single layer and multi-layer

Figure 7.5 Surface morphologies due to different hatch spacing at $125 \mathrm{~W}$ power and 1000 $\mathrm{mm} / \mathrm{s}$ scan speed. 128

Figure 7.6 Pore formed due to different hatch spacing at $125 \mathrm{~W}$ power and $1000 \mathrm{~mm} / \mathrm{s}$ scan speed. 128

Figure 7.7 Pore number formed within single layer with different laser parameters ..... 130 Figure 7.8 Surface morphologies due to different hatch spacing at $125 \mathrm{~W}$ power and 1000 $\mathrm{mm} / \mathrm{s}$ scan speed.

Figure 7.9 Pores formed due to hatch spacing of $122 \mu \mathrm{m}$ and $152 \mu \mathrm{m}$ at $125 \mathrm{~W}$ and 1000 $\mathrm{mm} / \mathrm{s}$. 131

Figure 7.10 Number of pores formed at different levels of hatch spacing. 133

Figure 7.11 Average pore diameter formed with $150 \mathrm{~W}$ at different scan speed and hatch spacings. 134

Figure 8.1 (a) CAD model of the specimen (unit in $\mathrm{mm}$ ) and (b) Arrangement of the single tracks on top of the semi-cylinder base.

Figure 8.2 (a) Cylinder samples fabricated on the build plate, and (b) Bruker 1173 microCT scanner setup. 142 Figure 8.3 Simulation domain used to perform a $250 \mu \mathrm{m}$ long single-track simulation. 143 Figure 8.4 Temperature dependent material properties of Inconel 625[25]. 144 Figure 8.5 (a) CT scanned cylinder after reconstruction, (b) Transverse cross section, and (c) Modification of the transfer function to remove the powder particles and observe the single tracks 145 


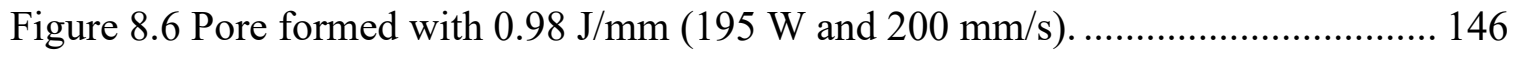

Figure 8.7 Pore volume and pore number from different LEDs.............................. 147

Figure 8.8 Average pore diameter and depth...................................................... 148

Figure 8.9 Transverse melt profile from different LEDs....................................... 149

Figure 8.10 Track width and depth measured from the transverse profile of different

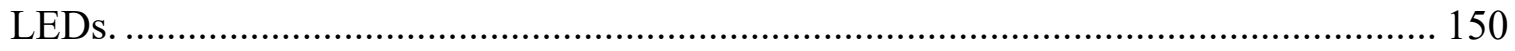

Figure 8.11 Pore volume and pore number from different power and speed when (a) LED

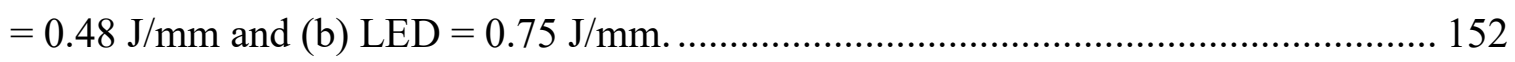

Figure 8.12 Transverse melt profile obtained from $\mathrm{LED}=0.48 \mathrm{~J} / \mathrm{mm}$ single tracks. ...... 153

Figure 8.13 The effect of normalized enthalpy on the non-dimensional depth and depth to width ratio. 155

Figure 8.14 Longitudinal melt profile obtained from $L E D=0.48 \mathrm{~J} / \mathrm{mm}$ and $0.75 \mathrm{~J} / \mathrm{mm}$ single tracks. 156 Figure 8.15 Temperature profile and the melt pool shape when laser has travelled 100 $\mu \mathrm{m}, 150 \mu \mathrm{m}, 200 \mu \mathrm{m}$ and $250 \mu \mathrm{m}(\mathrm{LED}=0.48 \mathrm{~J} / \mathrm{mm})$. 158

Figure 8.16 Figure 16. Depth to half width ratio comparison between simulation and

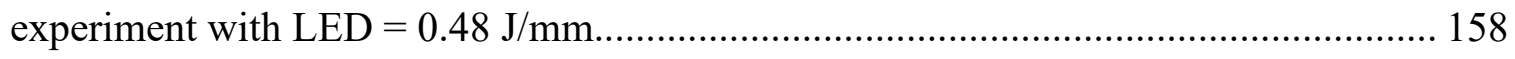

Figure 9.1 Single tracks fabricated on top of the semi-cylinder base ......................... 165 Figure 9.2 Design and dimensions of the miniature flowmeter and the setup used to obtain the angle of repose. 167 Figure 9.3 (a) Experiment and of repose used to calibrate cohesion energy density in (b) DEM simulation. 167

Figure 9.4 DEM setup to spread a layer of powder. 168 
Figure 9.5 Simulation domain used for $195 \mathrm{~W}$ and $800 \mathrm{~mm} / \mathrm{s}$. 169

Figure 9.6 Temperature dependent material properties of Inconel 625[18]. 169

Figure 9.7 (a) Surface profile, and (b) longitudinal and transverse 2D profile of $1 \mathrm{~mm}$ single track formed with $195 \mathrm{~W}$ and $800 \mathrm{~mm} / \mathrm{s}$. 171

Figure $9.82 \mathrm{~mm}$ single track surface profile and longitudinal 2D profile formed with 195 $\mathrm{W}$ and $800 \mathrm{~mm} / \mathrm{s}$ showing three zones. 172

Figure 9.9 (a) Surface profile, and (b) longitudinal and transverse 2D profile of $1 \mathrm{~mm}$ single track formed with $195 \mathrm{~W}$ and $400 \mathrm{~mm} / \mathrm{s}$. 173

Figure $9.102 \mathrm{~mm}$ single track surface profile formed with $195 \mathrm{~W}$ and $400 \mathrm{~mm} / \mathrm{s}$ showing three zones 174

Figure 9.11 Longitudinal melt profile obtained with $195 \mathrm{~W}$ and $800 \mathrm{~mm} / \mathrm{s}$. 175

Figure 9.12 Longitudinal melt profile obtained with $195 \mathrm{~W}$ and $400 \mathrm{~mm} / \mathrm{s}$. 176

Figure 9.13 Melt flow during the single-track scanning process. 178 Figure 9.14 (a) Top view of the melt profile formed with $195 \mathrm{~W}$ and $800 \mathrm{~mm} / \mathrm{s}$ and (b) Track profile compared at the free surface between experimental and simulation. 179 Figure 9.15 Top melt profile obtained for $2 \mathrm{~mm}$ simulation with $195 \mathrm{~W}$ and $800 \mathrm{~mm} / \mathrm{s}$. 180

Figure 9.16 Development of melt pool: 2D temperature contours obtained at the interface between substrate and powder. 181

Figure 9.17 Transverse melt profile obtained for experiment and simulation with $195 \mathrm{~W}$ and $800 \mathrm{~mm} / \mathrm{s}$ 182

Figure 9.18 Track profile compared at the free surface between experimental and simulation single track formed with $195 \mathrm{~W}$ and $400 \mathrm{~mm} / \mathrm{s}$. 183 
Figure 9.19 Development of melt pool: 2D temperature contours obtained at the interface between substrate and powder for $195 \mathrm{~W}$ and $400 \mathrm{~mm} / \mathrm{s}$. 184

Figure 9.20 (a) Top view of the $2 \mathrm{~mm}$ single track formed with $195 \mathrm{~W}$ and $400 \mathrm{~mm} / \mathrm{s}$, and (b) Transverse melt pool obtained from the experiment and simulation. 185

Figure 10.1 Schematic of the constant energy input in the scan lines during raster scanning. 190

Figure 10.2 (a) Single layer designed on top of the semi-cylindrical base, and (b) Single layer samples fabricated using EOS M270. 191

Figure 10.3 The working of the powder spreading process during LPBF process. 193

Figure 10.4 Consideration of actual powder height for first layer and second layer DEM simulation. 193

Figure 10.5 . Simulation domain used for $0.5 \mathrm{~mm}$ scan length simulation. 194

Figure 10.6 Temperature dependent material properties of Inconel 625 [19]. 195 Figure 10.7 (a)Multi-tracks, and (b) Multi-layers formed with $120 \mu \mathrm{m}$ hatch spacing. . 197 Figure 10.8 Multi-track profiles formed with $195 \mathrm{~W}, 750 \mathrm{~mm} / \mathrm{s}$ and $120 \mu \mathrm{m}$ at different scan lengths 198

Figure 10.9 Surface morphology obtained with multi-track and multi-layer formed with $195 \mathrm{~W}, 750 \mathrm{~mm} / \mathrm{s}$ and $120 \mu \mathrm{m}$. 199

Figure 10.10 (a) Location of the three sections, and transverse melt pool boundary from (b) slice 1, (c) slice 2, and (d) slice 3 of $0.5 \mathrm{~mm}$ scan length formed with $195 \mathrm{~W}$ laser power, $120 \mu \mathrm{m}$ hatch spacing and scanning speeds of $1500 \mathrm{~mm} / \mathrm{s}$ (left), $750 \mathrm{~mm} / \mathrm{s}$ (middle), an . 200 
Figure 10.11 Transverse melt pool boundary of $1.0 \mathrm{~mm}$ scan length from (a) slice 1, (b) slice 2, and (c) slice 3 formed with $195 \mathrm{~W}$ laser power, $120 \mu \mathrm{m}$ hatch spacing and scanning speeds of $1500 \mathrm{~mm} / \mathrm{s}$ (left), $750 \mathrm{~mm} / \mathrm{s}$ (center), and $375 \mathrm{~mm} / \mathrm{s}$ (right). 202

Figure 10.12 Micrographs of two-layers formed with $195 \mathrm{~W}$ laser power, $120 \mu \mathrm{m}$ hatch spacing, (a) $1500 \mathrm{~mm} / \mathrm{s}$ (b) $750 \mathrm{~mm} / \mathrm{s}$, and (c) $375 \mathrm{~mm} / \mathrm{s}$. 203

Figure 10.13 Temperature contour of the multi-track formed with $195 \mathrm{~W}$ laser power, $750 \mathrm{~mm} / \mathrm{s}$ scan speed, $120 \mu \mathrm{m}$ hatch spacing, and different scan lengths. 204

Figure 10.14 Temperature contour to observe the melt pool at different locations........ 206 Figure 10.15 Melt profile formed at different region along the scan direction. 207 Figure 10.16 Evolution of melt pool and vapor depression with $195 \mathrm{~W}$ laser power, 750 $\mathrm{mm} / \mathrm{s}$ scan speed, $80 \mu \mathrm{m}$ hatch spacing, and scan lengths of (a) $0.5 \mathrm{~mm}$ and (b) $1.0 \mathrm{~mm}$.

Figure 10.17 Variation in the actual powder layer thickness due to the variation in the surface height of the substrate layer. 210

Figure 10.18 Second layer melt pool boundary obtained with $195 \mathrm{~W}$ laser power, 750 $\mathrm{mm} / \mathrm{s}$ scan speed, $80 \mu \mathrm{m}$ hatch spacing and $0.5 \mathrm{~mm}$ scan length. 211

Figure 10.19 Second layer melt pool boundary obtained with $195 \mathrm{~W}$ laser power, 1500 $\mathrm{mm} / \mathrm{s}$ scan speed, $120 \mu \mathrm{m}$ hatch spacing and $0.5 \mathrm{~mm}$ scan length. 212 Figure 11.1 (a) CAD model of the specimen (unit in $\mathrm{mm}$ ), (b) Single tracks designed on top of previously deposited semi-cylinder, (c) Spatial distribution of the samples, and (d) Samples fabricated at different locations and different build heights..... 217 Figure 11.2 2D slices of $200 \mathrm{~mm} / \mathrm{s}$ formed at different locations (build height $=106.56$ $\mathrm{mm})$ 218 
Figure 11.3 Characteristics of pores formed with $195 \mathrm{~W}, 200 \mathrm{~mm} / \mathrm{s}$ and $195 \mathrm{~W}, 400 \mathrm{~mm} / \mathrm{s}$ (a), (b) pore number and volume, (c), (d) Average pore diameter, and (e), (f) average pore

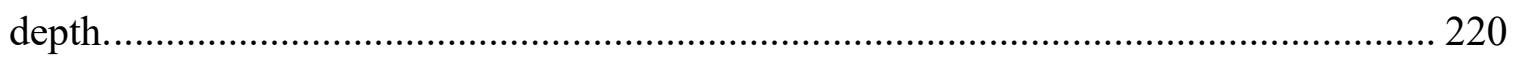

Figure 11.4 2D porosity comparison between micro-CT and micrograph.................. 221 


\section{CHAPTER 1 INTRODUCTION}

\subsection{Background and Motivation}

American society of testing and materials (ASTM) defines additive manufacturing (AM) as "a process of joining materials to make objects from 3D model data, usually layer upon layer, as opposed to subtractive manufacturing methodologies." [1]. There exist different AM technologies such as stereolithography (SLA), fused deposition modeling (FDM), inkjet printing (IJP), three-dimensional printing (3D-P), selective laser sintering (SLS), etc. [2]. Within the metal-based AM processes, laserbased additive manufacturing (LBAM) is a promising manufacturing technology that can be used for solid freeform fabrication [3]. Laser powder bed fusion (LPBF) process, also known as selective laser melting (SLM), is a layer by layer fabrication process which utilizes a laser to melt the powder particles in an inert atmosphere. Similarly, powder bed electron beam additive fabrication (PB-EBAF) is another powder bed AM technology that uses an electron beam instead of laser to melt the powder particles. Due to the use of an electron beam source, the melting must be carried out under vacuum. There are several differences between these two processes regarding the powder size used, penetration depth, etc. [4]. The schematics of these two processes are shown in Figure 1.1. 


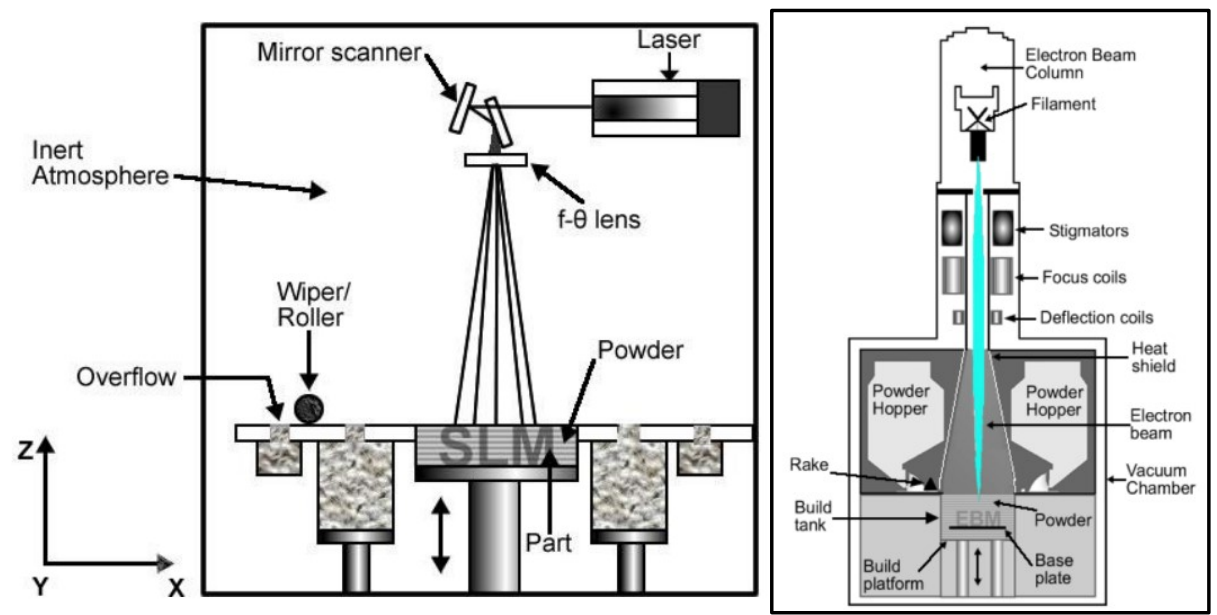

Figure 1.1 Schematic drawing of selective laser melting and electron beam melting system [5].

The quality of the single tracks during the PB-AM process mostly depends on laser parameters such as laser power, laser spot size, scan speed, and layer thickness. Besides, other variables such as hatch spacing, area of the island, ambient pressure, etc. also affect the quality during the part fabrication. These different parameters contribute differently, with some parameters affecting more than others. Porosity is one of the inherent process defects, which degrades the part mechanical performance, and therefore should be investigated. It is desired to reduce the part porosity, but the behavior of porosity formation should be understood to make the optimization process more efficient. Various studies have focused on the collective effect of power, scan speed, hatch spacing on the porosity level [5-7], but the individual effect of process parameters has not been investigated. The individual and collective effect of process parameters towards the porosity level would help minimize the porosity in a better way. However, it is difficult to understand the porosity formation mechanism by experiment alone. A high-fidelity model capable of predicting different melting modes such as partial melting, conduction 
melting, and keyhole melting needs to be developed to understand how the porosity forms and develops during the LPBF process.

\subsection{Research Objective and Scope}

Powder bed AM processes such as PB-EBAM and LPBF processes have shown its capability in freeform fabrication. The computational and experimental works have been performed to understand the process effect on the part quality and optimize the parameters to obtain the full density parts. Despite the intensive studies, there are still some areas that need to be understood and explored.

In this study, the effect of process parameters on the quality of surface and level of porosity is investigated. The AM machines are used to fabricate the samples using user parameters. A white light interferometer is used to measure the surface roughness, and a micro-CT scanner is used to characterize the pores. Besides, the numerical model is developed to understand the underlying physics that leads to surface roughness and porosity.

Chapter 3 focuses on the surface roughness of the PB-EBAM process. The surface morphology of the part formed with different process parameters are analyzed using a white light interferometer, and a numerical model is developed to predict the formation of the build surface.

A powder-scale thermo-fluid model is developed in Chapter 4 to understand the single-track formation during the LPBF process. Besides, the sequential powder addition 
algorithm is used to perform a multi-layer simulation and investigate the formation of a lack of fusion porosity.

Chapter 5 focuses on the characterization of the keyhole pores formed at different linear energy densities with Ti-6Al-4V powder. A micro-CT scanner is used to characterize and measure the pores formed inside the $12 \mathrm{~mm}$ single tracks formed with different laser power and scan speeds.

A high-fidelity powder scale numerical model is developed in Chapter 6, which is validated against the experiment. The model is utilized to understand the physics behind the keyhole pore formation. The interaction between the surface tension and recoil pressure in the keyhole wall is explored.

Chapter 7 investigates the keyhole pores and the lack of fusion pores formed during the multi-layer build. The optimum parameters obtained from a single-track study are used to observe the effect of hatch spacing on the porosity.

Chapter 8 explores the relationship between the linear energy density and melting mode during single-track fabrication. The LPBF process is used to fabricate the Inconel 625 single-track samples and establish the relationship between linear energy density and melt pool depth.

Chapter 9 discusses the formation of transient and steady regions during the single-track scanning in the LPBF process. The heat and mass transfer leading to the formation of a transient zone at the beginning and end of the single track is illustrated. 
Chapter 10 focuses on the residual heat effect on the melt pool characteristics. The effect of scan length and hatch spacing on the residual heat is studied.

Chapter 11 investigates the effect of build location on the keyhole porosity. The variability of the pores formed inside the single tracks at a horizontal plane and vertical plane is analyzed.

Chapter 12 discusses conclusions from this work and summarizes the future works. 


\section{CHAPTER 2 LITERATURE REVIEW}

The objective of this research is to develop a high-fidelity thermo-fluid model to predict the fluid flow and track formation during the powder bed-electron beam additive manufacturing (PB-EBAM) process and laser powder bed fusion (LPBF) process. Besides, this study aims to characterize pores and investigate the pore formation mechanisms in the LPBF process through experiments and simulations. This chapter provides the literature review on thermo-fluid modeling of metal AM processes, porosity measurement techniques, and pore characterization.

\subsection{Thermo-fluid modeling of PB-EBAM process.}

PB-EBAM is an additive manufacturing process that utilizes an electron beam to melt the powder particles in a vacuum environment. The process includes several complex physical phenomena such as heat transfer and melting, Marangoni effect, etc. which make it intricate to model. Qi et al. [8] utilized finite element analysis to investigate the heat transfer characteristics with different scanning strategies. The melting temperature was used as the criteria to check the presence of defects, as the number of elements that had not reached the melting temperature was defined as defects. Zah and Lutzmann [9] developed a transient 3D numerical model incorporating volumetric heat source to understand the process parameter effect on melt pool size during the PB-EBAM process. Fifteen simulation runs were carried out with different laser parameters (power 
and scan speed) to predict the melt pool length to width $(\mathrm{L} / \mathrm{W})$ ratio. Based on the $\mathrm{L} / \mathrm{W}$ ratio, laser parameters were selected for experimental evaluation. The authors emphasized the importance of considering fluid flow in the molten phase. Shen and Chou [10] studied the effects of porosity on the melt pool size in the PB-EBAM process by developing a numerical model in ABAQUS. The simulation results indicated that the increase in porosity led to a higher temperature and a deeper melt pool. In addition, the authors also explored the effect of bean diameter towards melt pool size. The larger beam diameter would reduce the maximum temperature leading to a lower cooling rate. Cheng et al. [11] developed a finite element model to predict the temperature distribution and melt pool size during the PB-EBAM process. The process parameter effect on the melt pool size was investigated both numerically and through experiments. For the experimental melt pool measurement, an infrared camera was used to obtain the apparent temperature during the PB-EBAM process [12], and the liquidus-solidus transition region was utilized to obtain the melt pool size. In the numerical model, the volumetric heat source was applied to simulate the heat transfer, and resultant melt pool sizes were measured and compared with the experiment. The temperature profile and melt pool size agreed well with the experiment. Galati et al. [13] used the Monte Carlo method to study the impact of an electron beam on the preheated powder bed. The simulation results showed that the electron energy rapidly decreases as a function of depth, and the penetration depth is negligible compared to the typical powder particle size. Furthermore, the laser spread of the beam would result in a greater effective diameter than the nominal focal diameter. Based on the findings, a new heat source was used to predict the thermal response and temperature distribution measured by Zah and Lutzmann [9] was used for 
the evaluation purpose. Besides, Jamshidinia et al. [14] developed a thermo-fluid model with a flat surface to understand the effect of surface tension gradient on the fluid flow during the PB-EBAM process. It was observed that the negative surface tension gradient of metal would result in an outward flow, which affected the melt pool size.

Flat surface simulations can predict the melt pool size, however, the flow behavior within the melt pool would require free surface modeling where the surface deforms due to melt flow. In this regard, Korner et al. [15] developed a 2D lattice Boltzmann model to study the single track formation during the PB-EBAM process, and the model helped explain physical mechanisms such as wetting, balling formation, etc. Scharowsky et al.[16] presented the vigorous moving melt pool and explained that the movement was caused by the surface tension force, which attracted the melt pool to already solidified areas. However, the 2D model would not provide complete information on the flow behavior during the melting process. Hence, Ammer et al. [17] introduced a 3D thermal lattice Boltzmann method, which utilized the volume of fluid (VOF) model to simulate the free surface formation during electron beam melting of powder particles. Yan et al. [18] used a discrete element method to simulate the powder bed distribution and utilized the powder characteristics in a thermo-fluid model to investigate flow behavior during single track formation. The key factors leading to the balling effect were found to be input energy and the layer thickness. 


\subsection{Thermo-fluid modeling of LPBF process.}

The LPBF process is a metal powder-based additive manufacturing (AM) process. During part fabrication, a laser source is applied to melt the distributed powder particles. As the powder particles are irradiated by the laser, metallic powders absorb the energy, which increases the powder temperature. When the temperature increases beyond the melting point, powder particles as well as substrate beneath start to melt, and melt flow occurs, which is primarily governed by the Marangoni effect. In addition, high energy input would further increase the melt pool temperature beyond the evaporation temperature and inducing the recoil pressure. This led to the formation of depression within the melt pool. The process has not been fully understood and numerical simulation is a good tool to understand the underlying physics. However, the process itself is complicated, and increasing the fidelity of simulation models would incur a high computational cost.

As the material melting occurs due to the absorption of laser, accurate modeling of the laser heat source is very important to predict the melt pool size correctly. Most studied have used the Gaussian heat source model presented in Figure 2.1[19]. Li and Gu [20] used a finite element method to simulate the temperature field during LPBF of AlSi10Mg powder. The results indicated that the cooling rate of the melt pool increased slightly as the power increased from $150 \mathrm{~W}$ to $300 \mathrm{~W}$. 


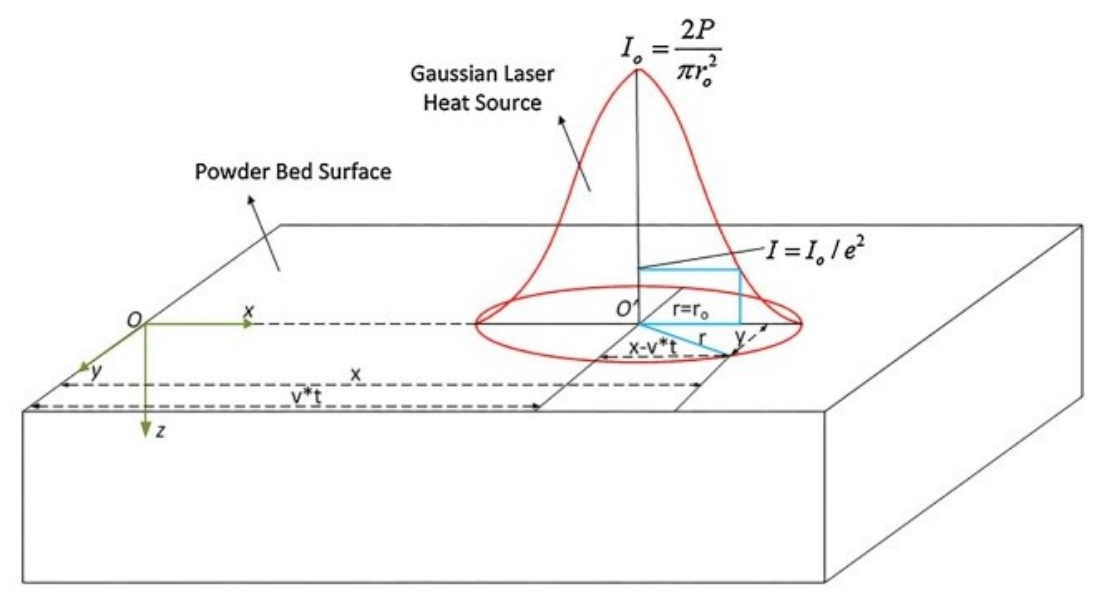

Figure 2.1 Gaussian laser heat source used for LPBF simulations.

Also, the residual heat from the first layer increased the maximum temperature, melt pool length, depth, and width during second layer scanning. Foroozmehr et al. [21] adopted the optical penetration depth of the laser beam while developing the FEM model. The model was used to predict melt pool dimensions of a single layer during LPBF of stainless steel 316L. Multiple track simulation showed that the melt pool dimensions reached a steady state after the third track although the melt pool size varied from the beginning of a track to its end and from the first track to the next. FEM models can predict the temperature and melt pool size. However, the LPBF process includes powder and surface deformation during the melting and solidification process. Dai and Gu [22] therefore utilized finite volume method with volume of fluid (VOF) technique to predict the surface deformation due to laser-material interaction. The simulation was able to predict surface pore formation as well as material stacking at different laser energy input per unit length (LEPUL). 
Furthermore, it is important to model the powder particles to predict melt pool and track formation. Xia et al. [23] developed a powder scale model to study the effect of hatch spacing on the surface quality of Inconel-718 parts fabricated by the LPBF process. Although the powder did not represent actual powder bed distribution, the model was able to show the effect of different hatch spacing on the material flow behavior. Khairallah and Anderson [24] developed a mesoscopic simulation model to predict the melt pool formation during the SLM process and the importance of including the stochastic nature of the powder bed has been discussed. Although the model was simplified by assuming constant surface tension of the molten metal, the simulated melt pool height and width were close to the experimental result. However, the track depth is underpredicted in the simulation as the Marangoni effect and recoil pressure have been neglected. Lee and Zhang [25] developed a high-fidelity model by including physics such as the Marangoni effect and recoil pressure, which are important to correctly predict the melt flow during the SLM process. The recoil pressure due to evaporation causes significant depression which enables the model to accurately predict melt pool depth as well. Besides, the transverse cross-section profile of the molten pool was found to be relatively symmetric about the center of the laser. Similarly, Wu et al. [26] demonstrated that the recoil pressure triggered keyhole formation significantly affects the flow behavior which in turn affects the track morphology. Melt pool predicted by recoil pressure is deeper and narrower compared to the identical model without the recoil pressure. A similar Multiphysics model has been applied to compare the melt pool formation between two different materials: steel and molybdenum [27]. The melt pool 
size predicted for molybdenum is much smaller than steel due to its higher thermal conductivity.

Besides finite element and finite volume techniques, a meshfree smoothed particle hydrodynamics (SPH) method has been used to understand the temperature distribution and flow behavior during laser scanning. Russel et al. [28] developed a 2D SPH model to study the effect of laser power on the surface roughness. It was observed that higher power leads to greater surface roughness and material sputtering. Similarly, Weirather et al. [29] developed a 3D multiphase model using a weakly compressible SPH approach and incorporated physical effects such as viscous force, surface tension, thermo-capillary forces, melting and solidification, etc.

\subsection{LPBF porosity measurement.}

Pores formed during the process may be characterized as keyhole pores and metallurgical pores [30]. These pores, if present within the SLM parts, would degrade its part performances[31-33], and therefore part optimization by porosity reduction is desired. Leuders et al. [33] explained that pores within SLM samples have a drastic effect on the fatigue behavior and significant extension of the crack initiation phase can be achieved by reducing the porosity. In addition, the pore location and pore size are also critical to the stress concentration, and pores near the surface are more critical even if they are small in size [34].

Pore formation during the SLM process is affected by the energy input, which is collectively defined by the energy density and is a function of laser power, scan speed, 
hatch spacing, and layer thickness. Gong et al. [35] investigated part quality based on different energy densities and demonstrated that process window in SLM of Ti-6Al-4V might be divided into three groups: insufficient melting, conduction melting, and keyhole melting. When the energy density is very high, the temperature within the melt pool exceeds the boiling point leading to material evaporation. The material evaporation would generate a recoil pressure, and huge depressions would form within the melt pool known as a keyhole. Such melting is known as keyhole-mode melting. As the energy density reduces, keyhole formation is not observed, and the mode of melting is called conduction melting [36]. The partial melting occurs with a further reduction of energy density, which leads to balling behavior and discontinuity in the track formation.

A few investigations towards part porosity developed during the SLM process has been carried out. Ponnusamy et al. [6] performed a statistical analysis to investigate the effect of various process parameters such as laser power, layer thickness, etc. towards porosity. Besides, various studies have been conducted to characterize porosity using Xray tomography methods. Slotwinski et al. [7] utilized X-ray computed tomography (XRCT) to measure porosity on additively manufactured cobalt-chrome (Co-Cr) samples. It has been shown that the XRCT technique can help determine the pore distribution within the sample as well as pore morphology. However, the accuracy of the representation would depend on the resolution of the system. Similarly, Zhou et al. [37] used Synchrotron radiation micro-CT to observe the defects formed within SLM parts. It was concluded that such an imaging technique is a feasible method to accurately obtain 3D images of SLM defects. Furthermore, Ziółkowski et al. [38] explained the advantage of nondestructive XRCT over traditional metallographic cross-sectional analysis. 
Siddique et al. [34] used a voxel resolution of $4.8 \mu \mathrm{m}$ to scan the SLM specimen. In addition, a two-dimensional metallographic study was also performed to calculate the porosity and it was found that porosity calculated by metallographic study and threedimensional tomography had no significant difference considering the critical pores. Kim et al. [39] used X-ray microtomography to study the track changes in the morphology of SLM Ti porous structures at a different stage of post-laser melting production. It has been demonstrated that $\mu \mathrm{CT}$ can be used not only for 3D quantification but also as a feedback mechanism to make improvements on initial designs. Van Bael et al. [40] used XRCT as a feedback mechanism to improve the geometrical and mechanical controllability of selective laser melted Ti-6Al-4V porous structures. The mismatch between designed and measured properties such as pore size, strut thickness and volume, porosity from the first run were integrated into the second run which helped minimize the mismatch. Kasperovich et al. [5] presented the correlation between process parameters and porosity formation during SLM fabrication of Ti6A14V parts. The porosity volume fraction can be reduced by the optimization of process parameters since two types of void defects are observed at excessive or insufficient energy densities. With excessive energy input, circular/spherical pores are formed whereas insufficient energy density resulted in elongated, narrow crack-like voids. 


\section{CHAPTER 3 A BUILD SURFACE STUDY OF POWDER-BED ELECTRON BEAM ADDITIVE MANUFACTURING BY 3D THERMO-FLUID SIMULATION AND WHITE-LIGHT INTERFEROMETRY}

\subsection{Introduction}

Additive manufacturing (AM) is a relatively novel process in which parts are built in a layer by layer fashion. Powder Bed Electron Beam Additive Manufacturing (PBEBAM) is a powder bed AM process that uses electron beam to selective melt the powder. Firstly, the metal powder is spread over the bed inside vacuum chamber which is then followed by preheating, contour melting and hatch melting. This process is continued until final desired part is built. Due to its capability, PB-EBAM has been utilized to produce complex components which are rather difficult, if not impossible to make by conventional manufacturing processes while maintaining full density. In addition, the ability of PB-EBAM process to directly fabricate metallic parts can significantly accelerate the product design and development.

Among other parameters, temperature is a very important process response of a PB-EBAM technology. Several numerical studies have been carried out to predict the thermal response and flow behavior of melt pool during the PB-EBAM process. Mahale [41] developed a 3D PB-EBAM model with temperature dependent Al 7075 
material properties and applied a point heat source using finite element method. A reliable estimate of temperature was obtained as a result. However, the point source may not be an actual representation of the heat source in PB-EBAM process. Therefore, Zäh and Lutzmann [9] developed a PB-EBAM 3D model incorporating volumetric heat source. Different combination of beam power and scanning speed were used to study their effect in resultant melt pool characteristics. During the modelling process, it is desired to have temperature dependent material properties. But, the thermal conductivity of liquid Ti-6Al-4V, which is the most popular material in PB-EBAM process, is not easily available over various temperature ranges. Therefore, effective estimate of thermal conductivity, which would include the effect of convection heat transfer in the pure thermal model, may be a possible approach to include the convection heat transfer in a pure thermal model [42]. Cheng et al. [11] developed a 3D thermal model using effective thermal conductivity to investigate the process temperature resulting from different scan speed, beam diameter and beam current. The simulation results: temperature, melt pool length and width were in good agreement with the experiment. In addition, Jamshidinia et al. [14] numerically studied the process temperature and fluid flow by developing a 3D thermal and thermo-fluid models. It is observed that the negative surface tension gradient of Ti-6Al-4V material resulted in outward flows which affected the temperature distribution and melt pool size. The thermo-fluid model would result in wider and shallower melt pool due to upward and outward fluid flow. This phenomenon of fluid flow at the interface due to surface tension gradient is known as Marangoni effect. The outward flow induced by Marangoni effect after melting and the formation of surface have been presented in Figure 3.1. 

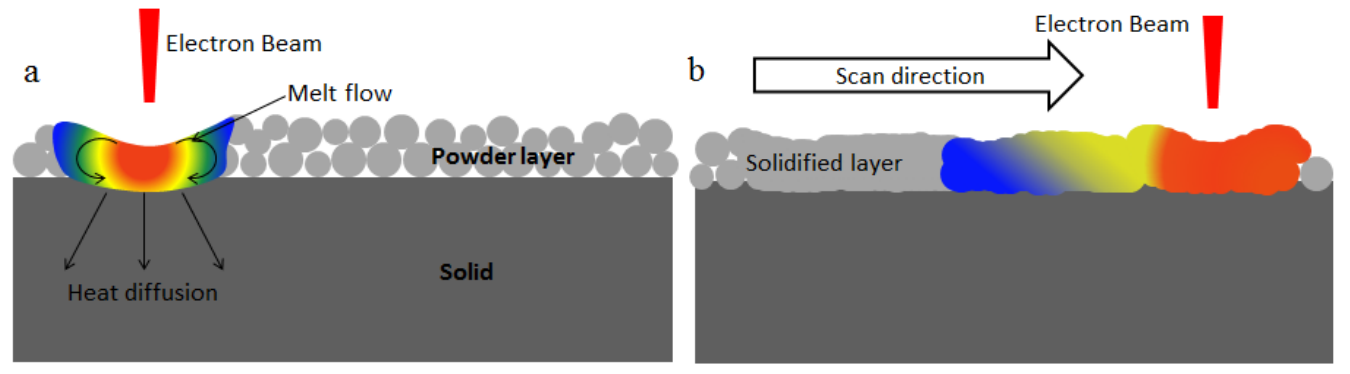

Figure 3.1 (a) Melting of powder immediately after beam strikes powder layer and (b) solidification after continuous melting.

Besides temperature gradient and mechanical properties, surface roughness is one of the issues related with PB-EBAM parts. The experimental studies suggest that fatigue life of parts fabricated by PB-EBAM process may depend on the quality of surface finish which in turn is the function of powder particle size, scan speed, layer thickness etc. [43]. Therefore, proper selection of process parameters will help improve the mechanical property along with decreasing the surface roughness. Various experimental studies have been conducted to understand the surface morphologies and roughness values for PBEBAM process at different process parameters. Kioke et al. [44] have presented the enlarged view of PB-EBAM specimen which shows the rippled appearance in the exterior. Although undesirable in most cases, these kinds of rippled surfaces may be helpful in some biomedical application where irregular surface is desired [45]. Rafi et al. [4] studied the surface formed by Selective Laser Melting (SLM) and PB-EBAM process. His results indicated that the "stairstep effect" is dominant in PB-EBAM process compared to SLM process due to formation of thicker layers. Safdar et al. [46] performed an in depth study of the effects of process parameter setting on the surface roughness of Ti-6Al-4V parts produced by PB-EBAM process. Based on the results, they concluded that all parts built by PB-EBAM process will have certain degree of roughness $\left(R_{a}\right)$ 
which ranges from 1-20 $\mu \mathrm{m}$ depending on the process parameter setting. In addition, the image taken from Scanning Electron Microscope (SEM) shows the formation of valleys parallel to the melt plate. Guo et al. [47] experimentally studied the effect of beam current on the material deposition during PB-EBAM process. They have shown that the lower beam current which is $4 \mathrm{~mA}$ result in porous surface with internal cavities whereas higher beam current of $12 \mathrm{~mA}$ result in dense and wavy surface. Strano et al. [48] presented the possibility of predicting the real surface roughness of SLM parts through mathematical model. The developed model to predict the roughness accounted for the presence of particles at the top surface and the stair step effect which makes it more appropriate compared to classic models. The results indicated that roughness predicted by mathematical model has good agreement with the experimentally observed roughness.

As the surface roughness depends on process parameter setting, experimental study of PB-EBAM process alone may not be sufficient enough to understand the physics associated with the surface formation during the process. Numerical simulation may be an alternative way to study the melt pool dynamics and formation of the top surface. However, there are always some challenges associated with modeling such complex phenomenon. Therefore, very limited numerical studies have been reported which shows the possibility to predict the surface morphology of AM process. Dai and Gu [22] have developed a 3D numerical model using finite volume approach to predict the surface morphology in SLM process. They have applied volume of fluid (VOF) model to predict the free surface formation due to thermo-capillary effect and pressure boundary condition at the interface. Although it is difficult to match surface formation to that of experiment, the numerical model was able to predict the formation of pores and material stacking at 
different laser energy input per unit length (LEPUL). Xia et al. [23] developed a powder scaled model using finite volume method to investigate the effect of hatch spacing $(\mathrm{H})$ on solidified surface quality of Inconel-718 parts fabricated by SLM process. Based on the experiment and simulation, it has been concluded that the lower hatch spacing of $40 \mu \mathrm{m}$ would result in migration of molten liquid towards the previous deposited material resulting in a stacking of molten liquid. The surface was flatter when hatch spacing of 60 $\mu \mathrm{m}$ was used. In the similar manner, this study focuses to predict the surface formation and roughness of PB-EBAM parts obtained with different process parameters. Continuum models treat powder particles as a continuous medium; however, they are discrete and randomly distributed. In this regard, Lattice Boltzmann Method (LBM) is used to model the powder particles. Some advances has been made to model SLM process using LBM models $[15,49]$. LBM method can incorporate features such as stochastic effect of randomly packed powder, capillary effect etc. which are not modelled in continuum approach. Besides in SLM process, LBM method has been applied to PBEBAM process as well. Bauereiß et al. [50] developed a mesoscopic model based on LBM to study the melting of powder particles and solidification.

In this study a 3D VOF model is developed to understand the surface formation and the resultant surface roughness in PB-EBAM process. A volumetric Gaussian heat distribution is applied to the powder and solid substrate by tracking the interface between the powder and the vacuum domain. A moving heat source with raster scanning pattern is applied at the free powder surface. Temperature dependent materials properties like density, thermal conductivity and specific heat capacity are incorporated as piecewise 
linear function in FLUENT while temperature dependent surface tension was applied through User Defined Function (UDF).

\subsection{Methodology}

\subsubsection{D model configurations}

A 3D model with domain size $8 \mathrm{~mm} \times 4 \mathrm{~mm} \times 2 \mathrm{~mm}$ is developed as shown in figure 3.2(a). The domain is further sub-divided into Ti-6Al-4V zone and vacuum zone: lower $1 \mathrm{~mm}$ in z-direction represents the Ti-6Al-4V zone and upper $1 \mathrm{~mm}$ represents the vacuum. Moreover, to represent the actual PB-EBAM process, $0.07 \mathrm{~mm}$ of powder layer acts as the newly spread powder over the previously deposited solid substrate whose depth is $0.93 \mathrm{~mm}$. The blue zone in Figure 3.2(a) represents the vacuum which has volume fraction 0 whereas the red zone with volume fraction 2 represent the solid Ti$6 \mathrm{Al}-4 \mathrm{~V}$ domain. The volume fraction of powder is 1 and the interface between vacuum and Ti-6Al-4V powder hold volume fraction value between 0 and 1 which is considered as the free surface. Continuum approach with VOF model to predict surface formation has been applied for SLM process which simulated only powder phase applying surface heat source [22]. However, the use of three phases with volumetric heat source makes the solution more appropriate to the experiment. To simplify the problem, the flow within melt pool is assumed to be laminar and incompressible.

A hexahedral mesh is defined over all domains which is preferred for VOF model.

In order to minimize the error occurring due to continuum approximation, very small mesh size of $30 \mu \mathrm{m} \times 30 \mu \mathrm{m} \times 14 \mu \mathrm{m}$ is defined in the powder layer and the interface so as to accurately track the deformed surface which has been presented in Figure 3.2(b). The 
size of the mesh increases farther away from the interaction region and the scan area as shown in figure 3.2(b) so as to reduce the number of elements and calculation time.
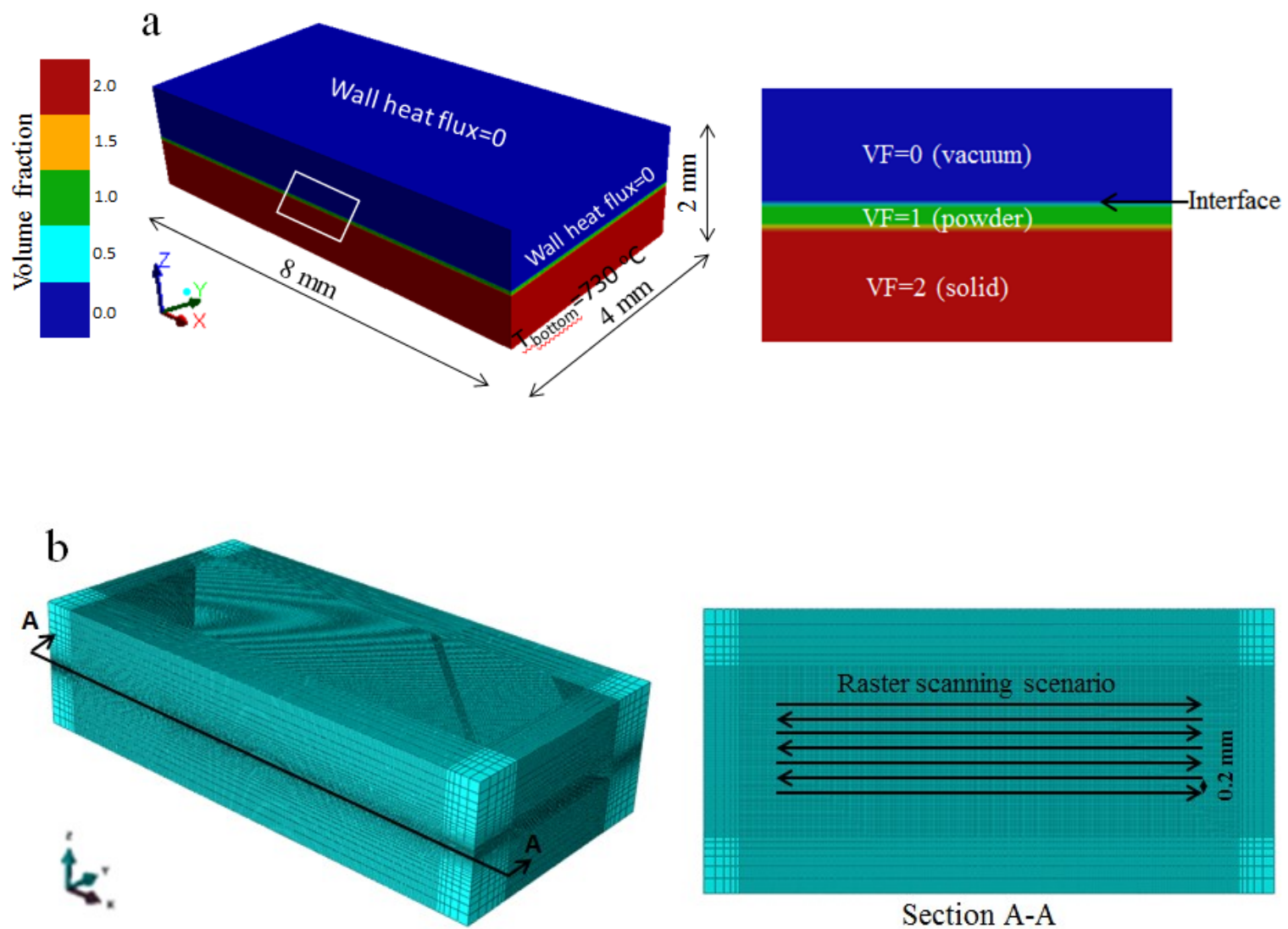

Section A-A

Figure 3.2(a) 3D model used for build surface analysis and detailed view for volume fraction information and (b) raster scanning applied at the top of powder layer

\subsubsection{Governing equations}

The basic three conservation equations: mass, momentum and energy are summarized below:

Mass conservation equation:

$$
\frac{\partial \rho}{\partial t}+\frac{\partial\left(\rho u_{i}\right)}{\partial x_{i}}=0
$$


where $\rho$ is the density, $\mathrm{t}$ is time and $x_{i}$ and $u_{i}$ are the distance and velocity in Cartesian co-ordinate system respectively.

Momentum Conservation Equation:

$$
\frac{\partial\left(\rho u_{j}\right)}{\partial t}+\frac{\partial\left(\rho u_{i} u_{j}\right)}{\partial x_{i}}=\frac{\partial}{\partial x_{i}}\left(\mu \frac{\partial u_{j}}{\partial x_{i}}\right)+S_{j}
$$

Where $u_{j}$ is the velocity in Cartesian direction, $\mu$ is the viscosity and $S_{j}$ is the source term in the momentum equation.

Energy Conservation equation:

$$
\frac{\partial(\rho H)}{\partial t}+\frac{\partial\left(\rho u_{i} H\right)}{\partial x_{i}}=\frac{\partial}{\partial x_{i}}\left(k \frac{\partial T}{\partial x_{i}}\right)+\dot{Q}(x, y, z)
$$

where $\mathrm{H}$ is the enthalpy, $k$ is the thermal conductivity and $\dot{Q}_{(x, y, z)}$ represents the electron beam heat source and its volumetric Gaussian distribution is defined as:

$$
\dot{Q}_{(x, y, z)}=\eta \times \frac{H_{S} \times I_{z}}{S}
$$

with

$$
\begin{array}{r}
I_{z}=\frac{1}{0.75}\left(-2.25\left(\frac{z}{S}\right)^{2}+1.5\left(\frac{z}{S}\right)+0.75\right) \\
H_{S}=\frac{2 W}{\pi d^{2}} \exp \left\{-\frac{2\left[\left(x-x_{S}\right)^{2}+\left(y-y_{s}\right)^{2}\right]}{d^{2}}\right\} .
\end{array}
$$

in which the parameters include electron beam absorption efficiency coefficient:

$\eta$, power: $W$, optical penetration depth: s, electron beam spot diameter: $d, x_{S}$ and $y_{s}$ : heat source center position. $\mathrm{H}_{\mathrm{s}}$ and $\mathrm{I}_{\mathrm{z}}$ are horizontal Gaussian distribution heat source and heat source magnitude decaying function in vertical direction, respectively. A user defined function (UDF) was developed to define the moving heat source which models equation 3.4 through 3.6. The source term was added to the energy equation to solve for the temperature. 


\subsubsection{Tracking of free surface}

In this study, VOF model is used to track the free surface. The VOF function is defined to indicate the topology of metal flow. The VOF equation is given as

$$
\frac{\partial F}{\partial t}+\vec{v} \cdot \nabla F=0
$$

where, $F$ represents the volume fraction and $\vec{v}$ represents the velocity vector. In this model, three phases have been used, vacuum, Ti-6Al-4V powder and solid Ti-6Al$4 \mathrm{~V}$. A free surface is the interface between powder and vacuum has the volume fraction between 0 and 1. During the scanning, due to continuous surface deformation, more than one cell in the interface within same cell column may have volume fraction between 0 and 1 . Therefore, it is necessary to identify the topmost cell with volume fraction between 0 and 1 as the free surface. This was achieved by using UDF and storing the cell information to the user defined memory which was later recognized as the unique free surface cell to apply the volumetric heat source.

\subsubsection{Boundary conditions}

The boundary condition at the interface is given as

$$
-k \frac{\partial T}{\partial t}=\dot{Q}_{(x, y, z) i n t}-\sigma \varepsilon\left(T^{4}-T_{\infty}^{4}\right)
$$

where, $\dot{Q}_{(x, y, z) \text { int }}$ is the heat source applied at the interface, $\sigma$ is the StefanBoltzmann constant and $\varepsilon$ is the emissivity which is used to account for the radiation through the top surface. Here, $T$ represents the temperature of interface and $T_{\infty}$ represents the chamber temperature. Since the PB-EBAM process takes place in vacuum chamber, 
there is no convection heat loss from the part surface and therefore not included in the boundary condition.

The Marangoni shear stress that results the outward flow of melt pool is given by:

$$
\tau=\frac{\partial \gamma}{\partial T} \frac{\partial T}{\partial n}
$$

where $\frac{\partial \gamma}{\partial T}$ represent the surface tension gradient and $\mathrm{n}$ is the vector tangential to the local free surface. Continuum surface stress (CSS) method is used to incorporate the tangential flow of the melt pool.

\subsubsection{Volumetric heat source}

The free surface of the model starts to deform once it reaches melting point after which the thermo-capillary force comes into effect. The application of heat source on a pre-defined flat surface may not be appropriate as the surface is continuously deforming. Therefore, the free surface needs to be tracked for reference to apply volumetric heat source. Surface heat source is also a possible way to define heat at the interface[22]. In this study, we have incorporated the volumetric heat source by tracking the interface so as to appropriately predict the thermal response of PB-EBAM process. The tracking of the free surface and application of volumetric heat source accordingly were accomplished through UDF. The heat intensity is assumed to have a Gaussian distribution horizontally and linear decay along the penetration depth as shown in equation 3.4. 


\subsubsection{Vacuum approximation}

The PB-EBAM process is carried out inside a vacuum chamber and an ideal vacuum environment may not be modelled in VOF model. The momentum equation is solved for the interface region which shares the portion of vacuum and Ti-6Al-4V material. Hence, the viscosity and density cannot be zero; otherwise the solution would become unphysical. Therefore, it was necessary to make approximation in order to define vacuum. The operating pressure was set to zero; density and viscosity were highly decreased to the range with which the converged solution would be obtained.

\subsubsection{Material properties}

Temperature dependent material properties are summarized in Figure 3.3. The graph shows that density, specific heat and thermal conductivity of Ti-6Al-4V have almost linear dependency with temperature and thus applied in fluent as piecewise linear function. The reduced thermal conductivity of powder has also been considered [11]. Meanwhile, temperature dependent surface tension was applied through user defined function. In addition, other physical properties of Ti6Al4V are summarized in Table 3.1.
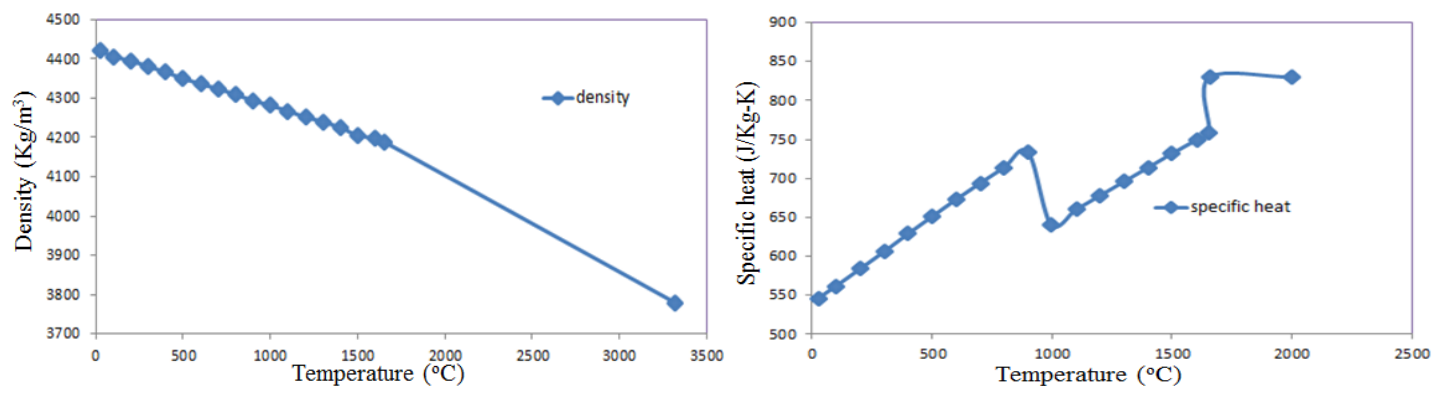


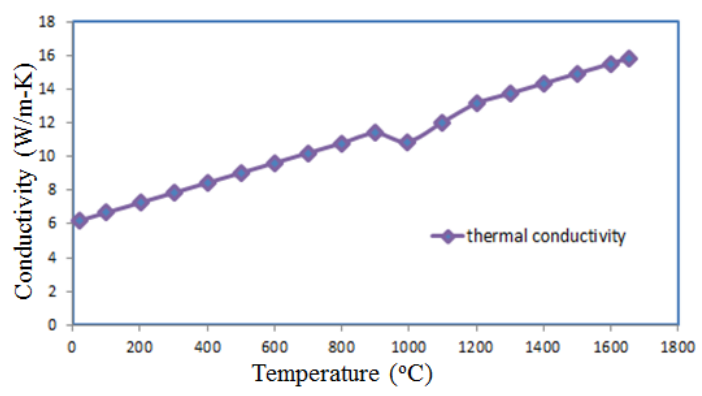

Figure 3.3 Temperature dependent density, specific heat and thermal conductivity of Ti6Al-4V [18-20].

Table 3.1 Properties of Ti-6Al-4V and process parameters used in model

\begin{tabular}{ll}
\hline Parameters & Values \\
\hline Solidus temperature, $T_{S}\left({ }^{\circ} \mathrm{C}\right)$ & $1605[51]$ \\
Liquidus temperature, $T_{L}\left({ }^{\circ} \mathrm{C}\right)$ & $1655[51]$ \\
Latent heat of fusion, $L_{f}(\mathrm{~kJ} / \mathrm{Kg})$ & $440[51]$ \\
Beam diameter, $\Phi(\mathrm{mm})$ & 0.65 \\
Hatch spacing $(\mathrm{mm})$ & 0.2 \\
Absorption efficiency, $\eta$ & $0.9[52]$ \\
Acceleration Voltage, $U(\mathrm{KV})$ & $60[53]$ \\
Powder layer thickness, tlayer $(\mathrm{mm})$ & 0.07 \\
Porosity, $\varphi$ & 0.5 \\
Preheat temperature, $T_{\text {preheat }}\left({ }^{\circ} \mathrm{C}\right)$ & $730[11]$ \\
Viscosity $(\mathrm{kg} / \mathrm{m}-\mathrm{s})$ & $0.049[55]$ \\
Surface Tension Gradient $(\mathrm{N} / \mathrm{m}-\mathrm{K})$ & $-0.00026[14]$ \\
Emissivity, $\varepsilon$ & $0.7[56]$ \\
Stefan-Boltzmann constant, $\sigma$ & $5.67 \times 10^{-8}$ \\
\hline
\end{tabular}

\subsection{Experimental analysis}

ARCAM PB-EBAM machine was used to build the samples using the system specific setting called Speed Function (SF), which controls the electron beam speed, current and the raster spacing during the part fabrication process to obtain the desired melt pool size [57]. Ti-6Al-4V parts with build height $30 \mathrm{~mm}$ were fabricated using various SF levels: SF20, SF36, SF50 and SF65. The speed function has been discussed 
in more detail in literature [12] and it has been indicated that the SF setting strongly affects the surface morphology and surface roughness.

The roughness of as obtained sample surfaces was measured using white light interferometer. The scan areas of $1.2 \mathrm{~mm} \times 1.2 \mathrm{~mm}$ were selected from different regions of the surface for the measurement. Average surface roughness $\left(\mathrm{S}_{\mathrm{a}}\right)$, surface root mean squared roughness $\left(\mathrm{S}_{\mathrm{q}}\right)$, maximum surface roughness $\left(\mathrm{S}_{\mathrm{t}}\right)$ etc. over the scan area are measured at the same time. The contour plot of a typical selected region from the surface along with measured surface statistics are depicted in Figure 3.4. Figure 3.5 shows the surface profile along the scanning direction and in transverse direction as measured by the white light interferometer passing through the point $\mathrm{P}$. The measured $2 \mathrm{D}$ profile is along the red line in scanning direction and blue line in transverse direction as shown in Figure 3.4. The average surface roughness $\left(\mathrm{R}_{\mathrm{a}}\right)$ along the scanning direction was found to be lower compared to that of transverse direction. In addition, the profile along transverse direction is semi-periodic with wavelength approximately equal to hatch spacing. PBEBAM process is a rapid melting and solidification process. During melting, the outward flow of melt pool may accumulate liquid material at the edges of the scan which then rapidly solidifies to form an elevated area on both sides of the scan track. As the hatch spacing of $0.2 \mathrm{~mm}$ was used for experiment, the elevated region repeats after $0.2 \mathrm{~mm}$ gap which can be clearly observed from Figure 3.5(b). 


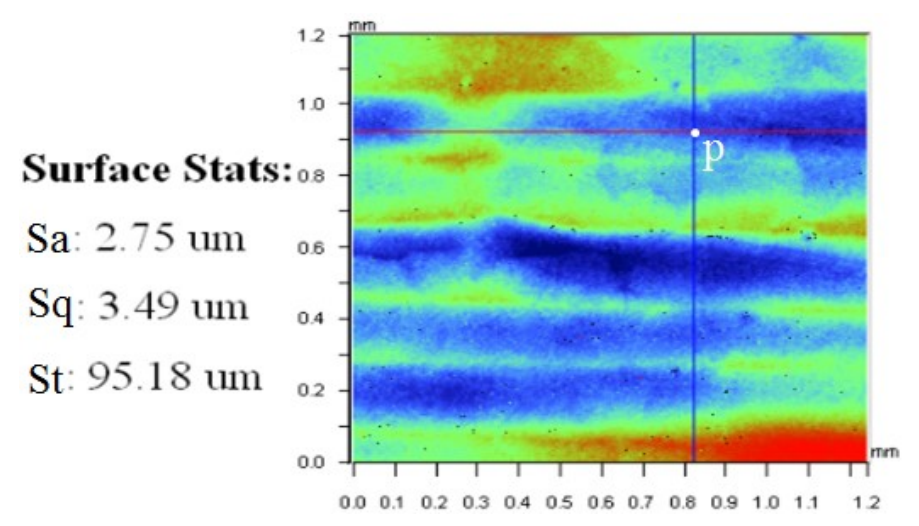

Figure 3.4 Contour plot as displayed by white light interferometer
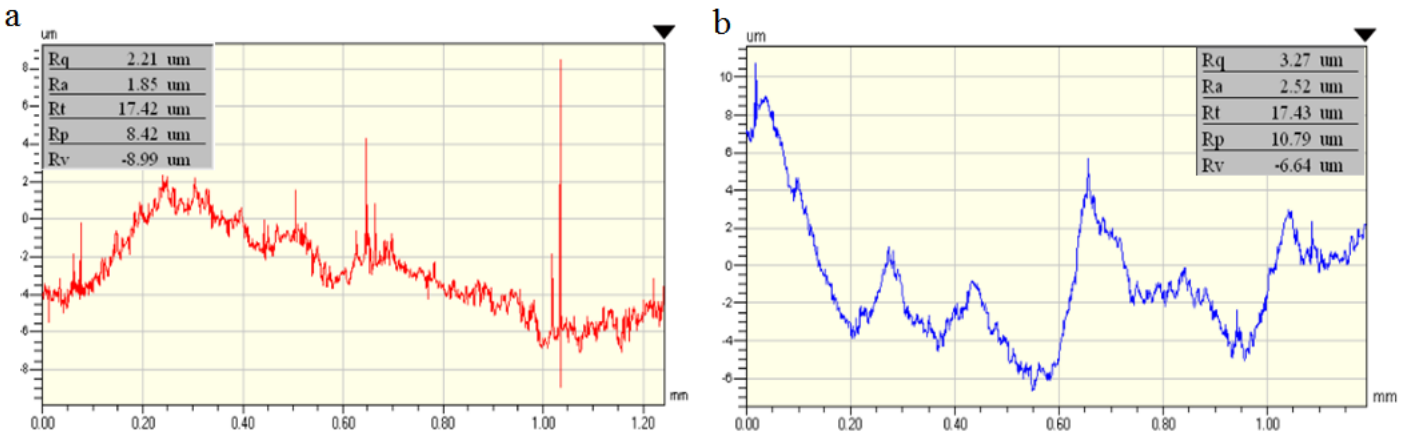

Figure 3.5 Typical 2D profile output from white light interferometer along (a) scanning direction and (b) transverse direction.

\subsection{Numerical simulation}

A 3D VOF model has been developed using ANSYS Fluent software. The volume fraction equation was solved along with energy and momentum equation to predict temperature, fluid flow and surface deformation. As the electron beam strikes the powder layer, the temperature of the powder rapidly increases, and it immediately melts the powder. Surface tension gradient then comes into effect which tends to move liquid Ti-6Al-4V from high temperature region to lower temperature region. This thermocapillary effect becomes dominant over viscous drag and begins to deform the surface. The magnitude of surface tension will vary within the melt pool and is maxima at the 
edges of the melt pool. The surface deformation study was carried out at different beam speeds.

\subsubsection{Typical simulation result}

For a given material, thermal response of the PB-EBAM process depend on process parameters and the magnitude of the fluid flow depends on the thermal gradient. The melt pool flow for liquid Ti-6Al-4V is from central high temperature region to surrounding lower temperature region due to its negative surface tension gradient as shown by the flat surface simulation [14]. However, in practice, the free surface in PBEBAM process undergoes continuous deformation during melting and solidification, and the resultant flow of the melt pool may be affected by the surface deformation. Therefore, in this study, VOF model is applied to predict the temperature on the deforming surface and resultant deformation due to melt flow. The typical simulation result, with temperature distribution and free surface formation along the first scan track for SF36 case is depicted in Figure 3.6. It is observed that the temperature distribution is symmetrical in the electron beam scan direction and the maximum temperature predicted is $2486.8{ }^{\circ} \mathrm{C}$ which is well above the melting point of Ti-6Al-4V. This ensures proper melting of powder material. As seen from Figure 3.6(b), there is a hump formation due to outward melt flow due to Marangoni effect at the edges of scanning region which may highly contribute in the eventual surface roughness of solidified free surface. We may also observe from Figure 3.6(c) that the surface ripples formation along the scanning direction may not be significant compared to the hump formation along the transverse direction. 
Temperature profile along the center line of first scan is illustrated in Figure 3.7 which exhibits the transition from powder to the liquid region. It is also perceived that the region of high thermal gradient is just outside of the electron beam and there is an extreme rise and fall of temperature near the beam center. Moreover, the temperature distribution along transverse direction at the beam center also shows higher gradient at the edges of the melt pool which would define the velocity distribution in the melt pool. Therefore, magnitude of velocity is higher at the regions with higher temperature gradient, due to higher surface tension force, as shown in Figure 3.8. In addition, the molten materials tend to move outward and this phenomenon continues along the scan track forming the elevated region in the edges of the melt pool.
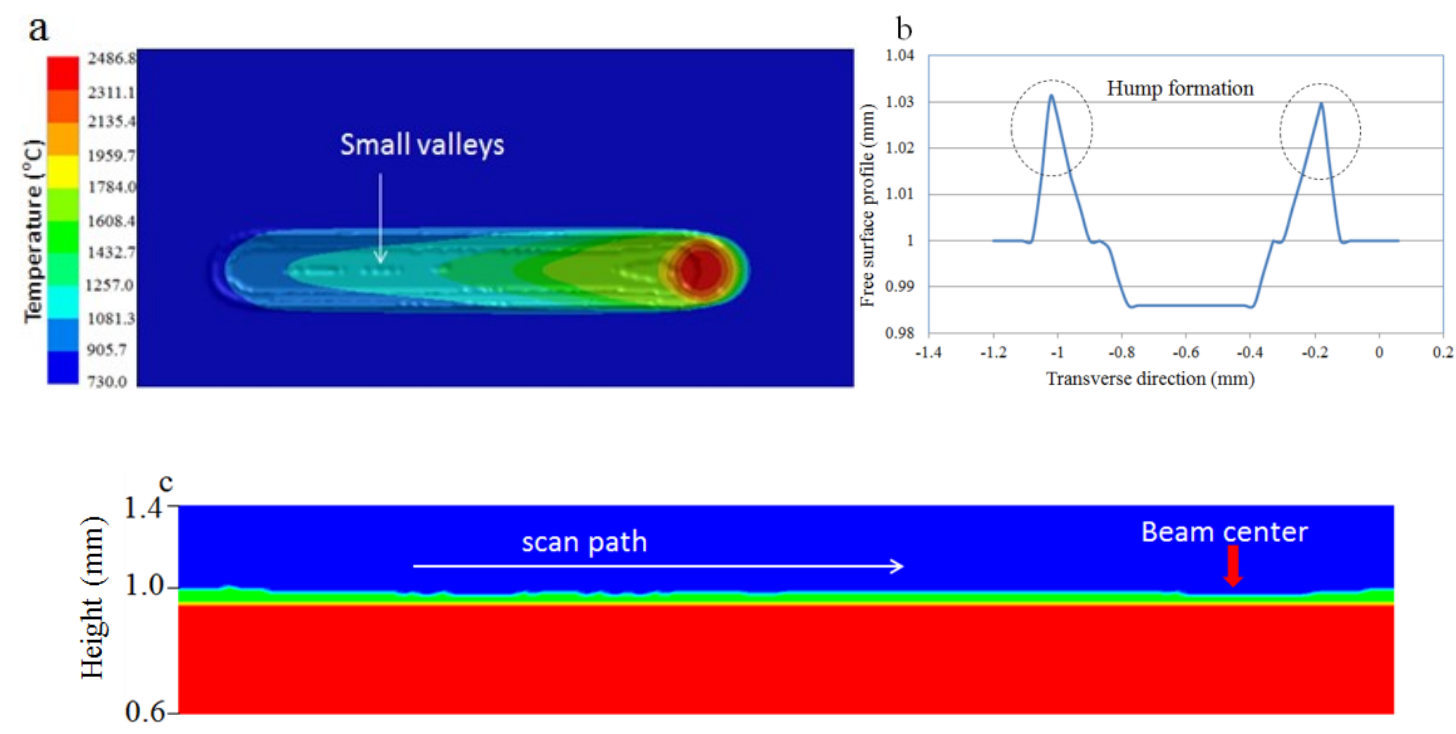

Figure 3.6 (a) Temperature distribution at the end of first scan track, (b) hump formation after solidification in transverse direction and (c) surface profile along scanning direction. 

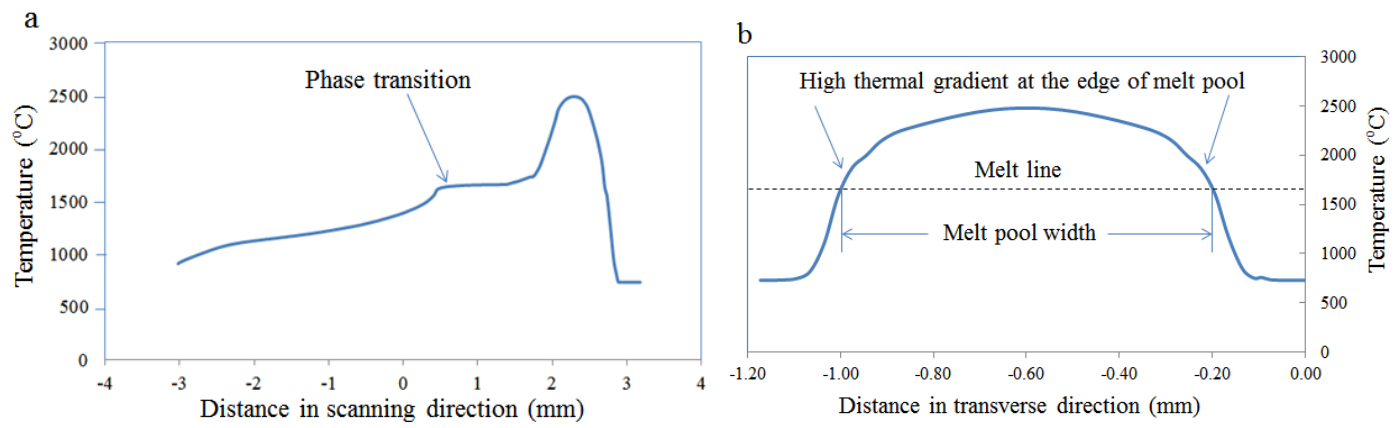

Figure 3.7 Temperature profile along (a) scanning direction and (b) transverse direction

The velocity profile at different sections of the melt pool has been summarized in Figure 3.8. The liquid metal tends to move upward and outward from the beam center which may define how the surface would form during PB-EBAM process. The velocity at the tail region of melt pool is lower which would reduce to zero as the beam moves away defining the solidified area.

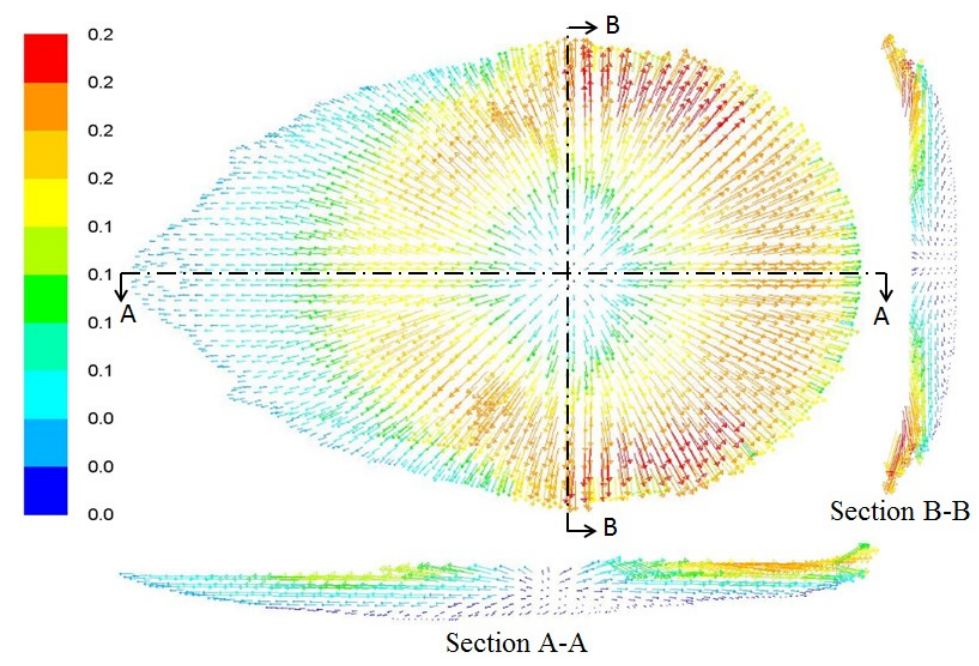

Figure 3.8 Velocity profile within melt pool

As the scan continues, the previously melted region begins to solidify due to heat loss from conduction and radiation. During the second scanning which is in opposite direction the lower elevated region from previous scanning may not be affected however 
much of the upper solidified elevated region is re-melted due to lower hatch spacing. Over the course of numerous back and forth scanning, the solidified elevated and shallow regions will from wave like structure. This wave like structure has noticeable crests and troughs which repeat after the length equivalent to the hatch spacing as indicated in Figure 3.9. Besides, it is also interesting to observe round shallower areas at the transition region where scan vector changes direction. These are the region where the electron beam strikes the longest resulting in longer heating which gives enough time for the melt pool to spread in larger area.

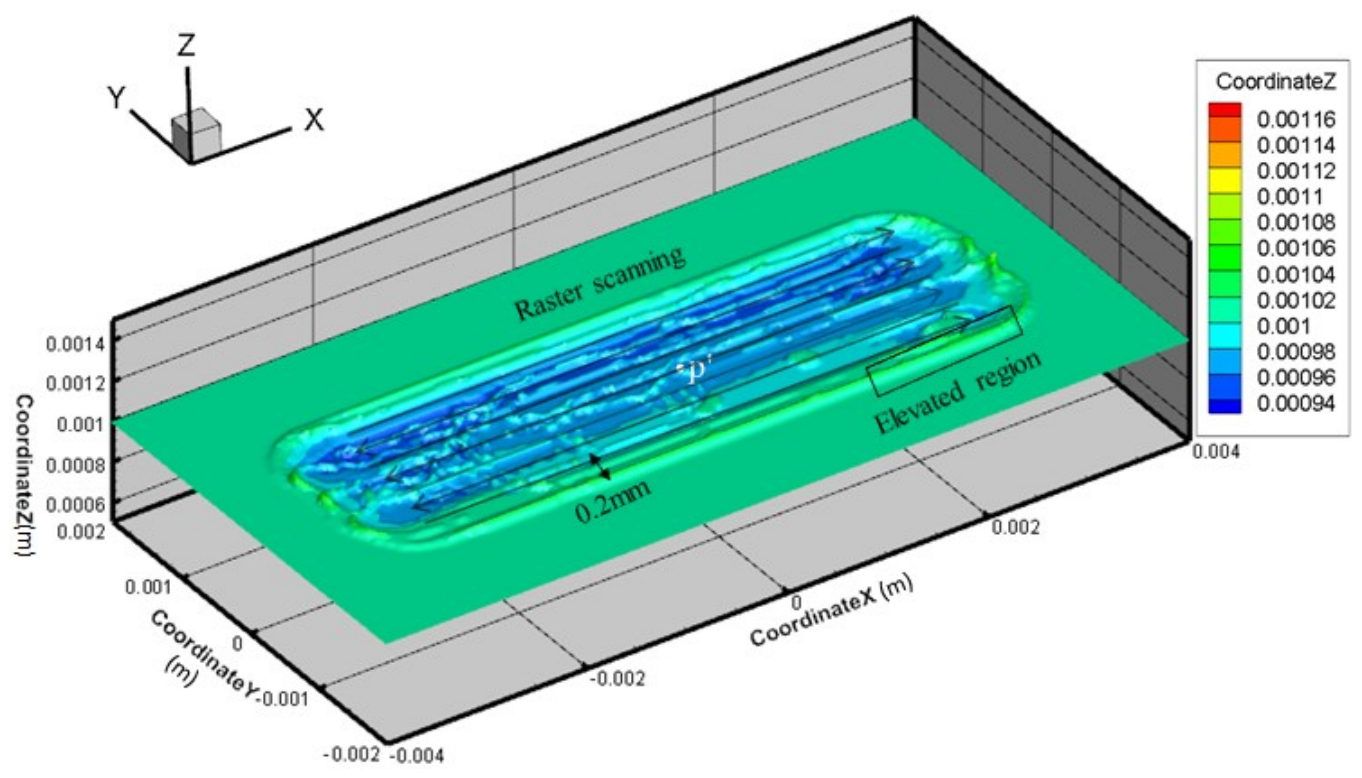

Figure 3.9 Surface morphology predicted for SF36 case.

The heat penetration of electron beam is higher than laser melting processes. When the powder layer is being melted, the applied heat is enough to melt the solidified layer below the scanning area which ensures proper interlayer bonding. Due to the fluid movement, the liquid formed from solid would mix with the liquid formed from the powder. Figure 10 which is a portion of cross section along the scanning direction shows 
the mixing and melt separation phenomena. Some portion of solid phase gets mixed with the powder as highlighted in the Figure 3.10(a). This phenomenon is observed more at the region where the electron beam changes direction as a region is exposed to heating for longer time and there is melt pool flow for longer time. Besides, it is also interesting to observe the melt separation. When the surface tension force is very high, the molten materials get ejected from the surface which is observed during the simulation and shown in Figure 3.10(b). The materials dropping at the nearby solidified region may also contribute to the final surface roughness.
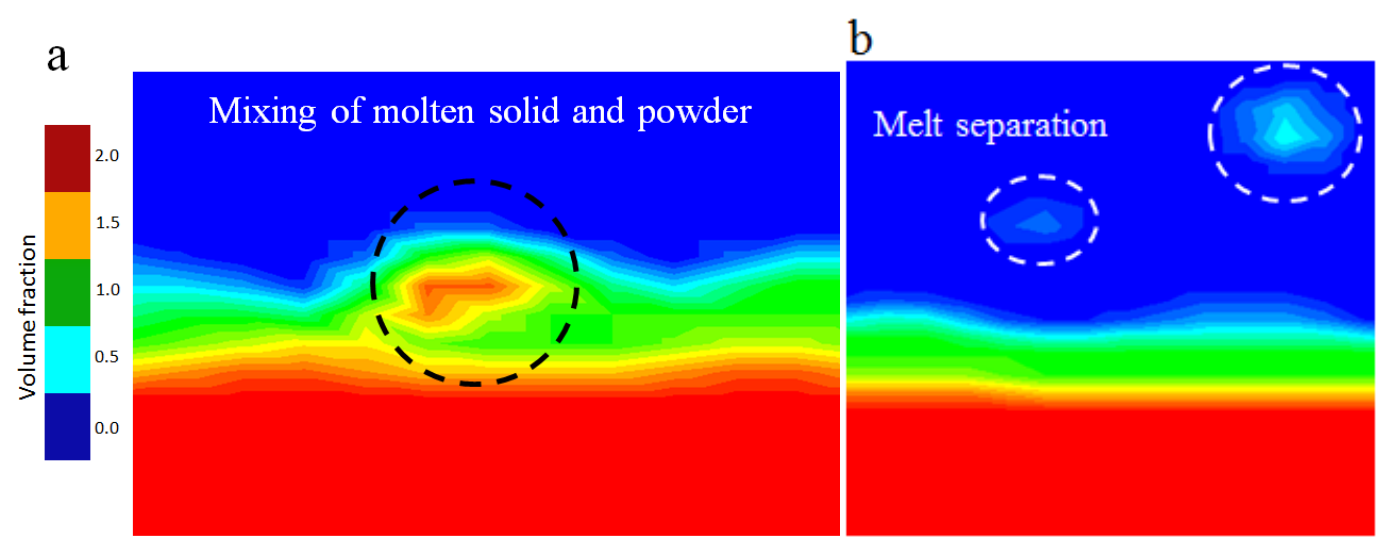

Figure 3.10 (a) Mixing of powder and solid particles after melting and (b) melt separation.

Figure 3.11 depicts the surface profile along the line in scanning direction and transverse direction passing through point $\mathrm{p}^{\prime}$, the origin, as shown in Figure 3.9. A central line is drawn for the average roughness analysis and the sampling length of $6 \mathrm{~mm}$ and 1.2 $\mathrm{mm}$ were chosen in the scanning and transverse direction respectively. The $\mathrm{R}_{\mathrm{a}}$ analysis over the obtained profile shows very little difference between scan and transverse direction, that is $5.2 \mu \mathrm{m}$ and $5.5 \mu \mathrm{m}$. However, we may say that the transverse direction has higher $\mathrm{R}_{\mathrm{a}}$ compared to the scanning direction. 
The surface profile along the scanning direction is rather irregular. In contrast, surface profile along transverse direction shows the repetition of elevated area after 0.2 $\mathrm{mm}$ which is in good agreement with the experimental result. It is also observed that the average height of the free surface is lower than $1 \mathrm{~mm}$. This may be due to shrinking of the material after melting. It is also apparent from the free surface profile that the surface height would decrease along the transverse direction due to accumulated shrinkage as a result of re-melting during melting. Considering the magnitude of shrinkage for a single layer, the overall shrinkage for the build part would be significantly higher.
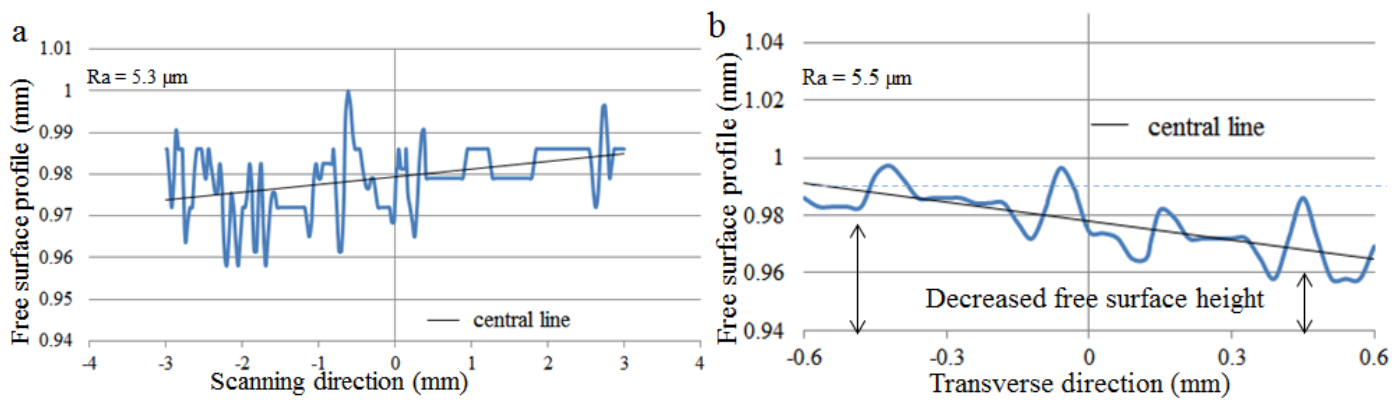

Figure 3.11 Surface height profile along (a) scanning direction and (b) transverse direction.

\subsection{Results and Discussion}

\subsubsection{Experiment results}

The stereoscopic images of different samples along with isometric images taken from white light interferometer have been portrayed in Figure 3.12. It is noticed that surface profile for SF20, SF36 and SF50 shows continuous track, whereas the surface of SF65 case has large porous regions which may be due to improper melting of powder particles; lower melt pool size may be a reason for poor surface formation. The surface 
height typically ranged from $-7.4 \mu \mathrm{m}$ to $11.6 \mu \mathrm{m}$ in SF $20,-11.9 \mu \mathrm{m}$ to $17.4 \mu \mathrm{m}$ in SF36, $-24.1 \mu \mathrm{m}$ to $59.5 \mu \mathrm{m}$ in SF50 and from $-231 \mu \mathrm{m}$ to $197 \mu \mathrm{m}$ in SF65 case. We may identify the scan tracks from the isometric view, the red zone indicates the elevated region and the blue zone represent the shallow region. However, specifically for SF65 case, the isometric view may not provide much information regarding the surface profile due to higher disturbance in the surface. We may also observe the decrease in height along the transverse direction from left to right for SF36 and SF50 cases which may be due to shrinkage. This phenomenon is also observed from the simulation result as shown in Figure 3.11(b).

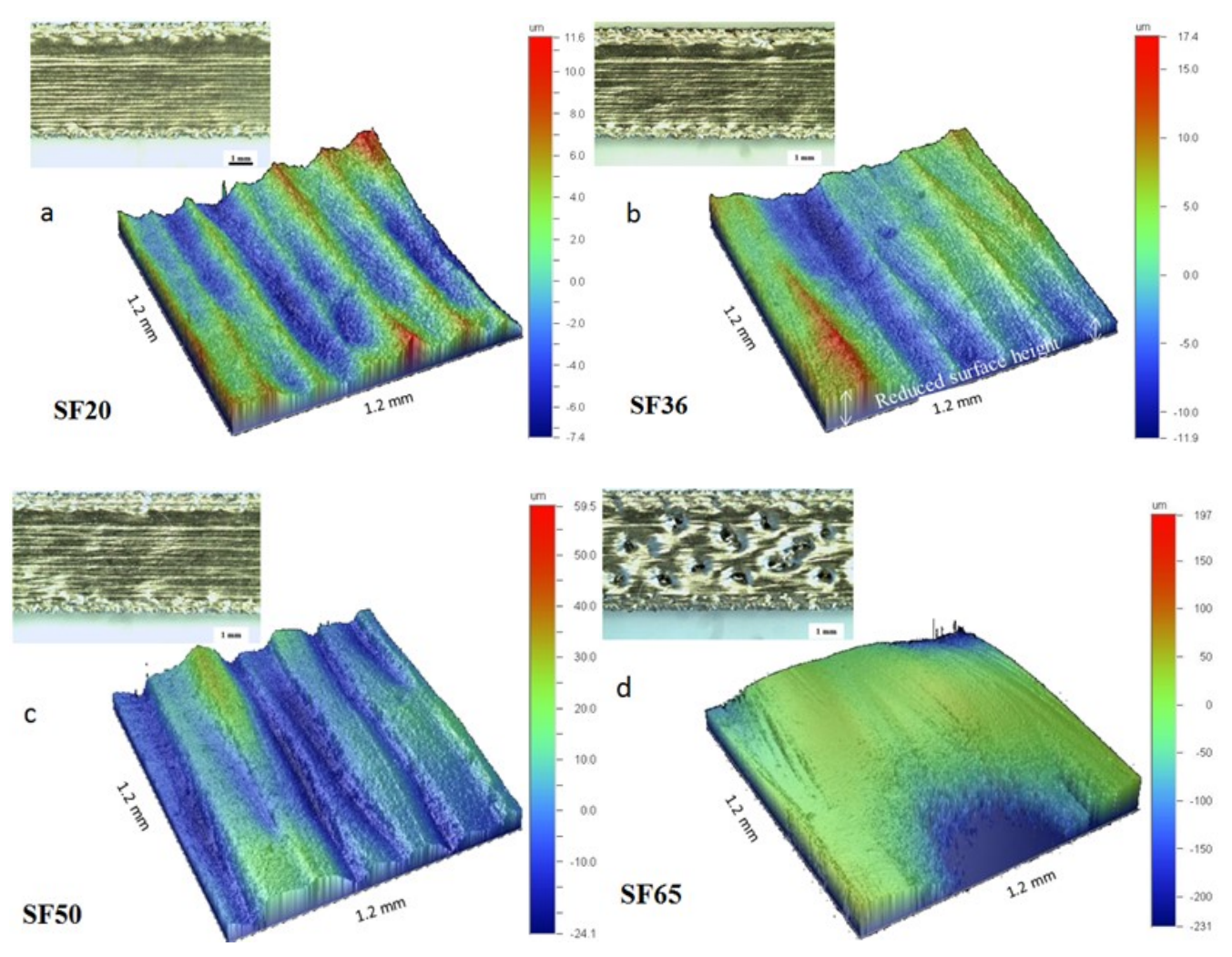

Figure 3.12 Stereoscopic image and isometric view of surface scanned taken from white light interferometer for (a) SF20, (b) SF36, (c) SF50 and (d) SF65 cases. 
Five scan areas are selected from the obtained samples for all cases, and the measured Ra values are summarized in Figure 3.13.

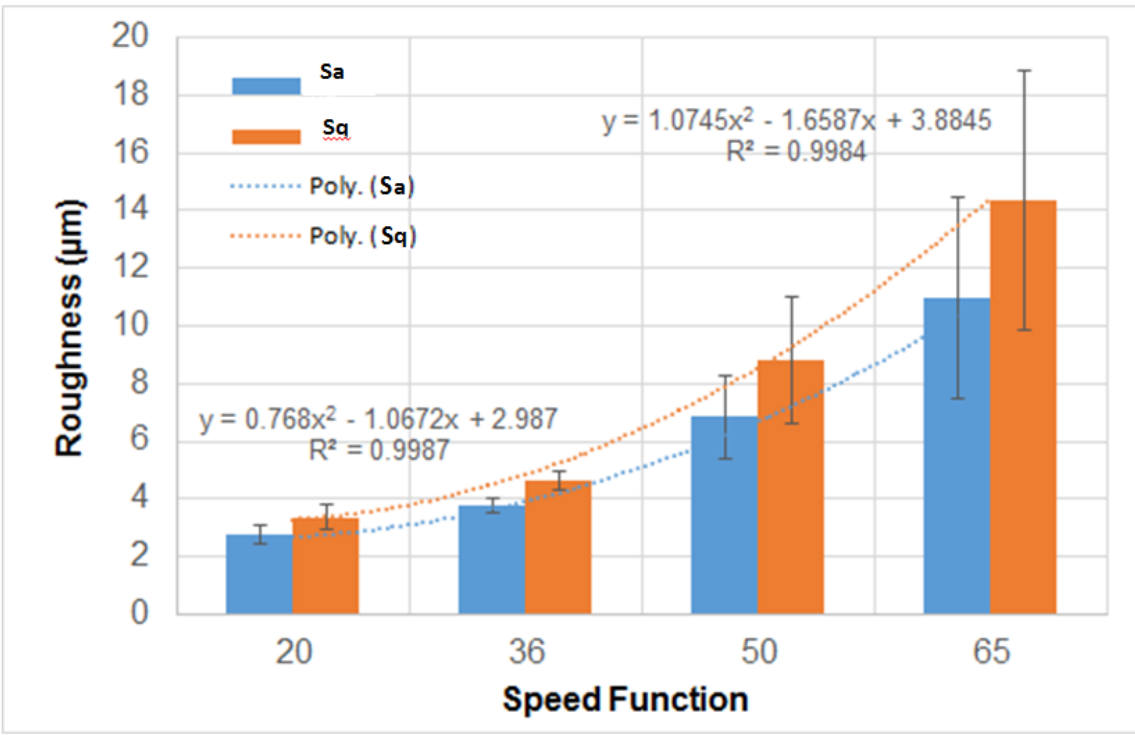

Figure 3.13 Average surface roughness for different SF cases.

The results indicate that average surface roughness depends on the beam speed and increases with speed function. In general, $S_{a}$ ranged from $3 \mu \mathrm{m}-11 \mu \mathrm{m}$ in average, with SF65 case having maximum surface roughness. When the beam speed increases the melt pool gets smaller as the powder layer gets heated by electron beam for shorter time. Therefore, powder particles may not be properly melted which may lead to insufficient distribution of melt pool, formation of pores and eventually higher surface roughness.

In addition to overall roughness, numerous points on the free surface were selected to obtain the $\mathrm{R}_{\mathrm{a}}$ at the scanning and transverse directions for all SF cases to understand how directions contribute to surface roughness. The roughness in scanning direction is consistently lower compared to that of transverse direction. This shows that the ripple formation in PB-EBAM process is not significant compared to the crest and trough formation in the transverse direction. The crests and troughs formed in the 
transverse direction repeats with respect to the hatch spacing. Therefore, hatch spacing may also play great role in overall surface roughness of PB-EBAM process.

\subsubsection{Simulation results}

\subsubsection{Melt pool characteristics}

The process parameters in accordance to different SF indices were used and simulations with different beam speed were carried out. These beam speed correspond to different Speed Function: SF20 $(481 \mathrm{~mm} / \mathrm{s})$, SF36 $(853 \mathrm{~mm} / \mathrm{s})$, SF50 $(1193 \mathrm{~mm} / \mathrm{s})$ and SF65 $(1595 \mathrm{~mm} / \mathrm{s})$. The melt pool for each case is summarized in Figure 3.14 which explains that the temperature distribution and resultant melt pool highly depend on the beam speed. Previous experiments conducted by Price et al. [12] showed that the melt pool length and width is the function of build height. For the same speed function, typically SF20, melt pool length was observed to decrease consistently with increasing build height. Moreover, maximum temperature, melt pool length and width decrease with increasing speed function. The simulation results show the similar trend. The maximum temperature predicted by free surface model for SF20, SF36, SF50 and SF65 cases were $2643{ }^{\circ} \mathrm{C}, 2487{ }^{\circ} \mathrm{C}, 2351{ }^{\circ} \mathrm{C}$ and $2322{ }^{\circ} \mathrm{C}$ and respectively which are in good agreement with the experimental results of $2550{ }^{\circ} \mathrm{C}, 2420{ }^{\circ} \mathrm{C}, 2350{ }^{\circ} \mathrm{C}$ and $2200{ }^{\circ} \mathrm{C}$.

The melt pool size decreases with increase in speed for same power input. The melt pool length predicted by the free surface simulation is $1.73 \mathrm{~mm}, 1.34 \mathrm{~mm}, 1.2 \mathrm{~mm}$, and $1.187 \mathrm{~mm}$ respectively for SF20, SF36, SF50 and SF65 cases. Considering the surface analysis for the top surface which has the height $30 \mathrm{~mm}$, the melt pools can be said to be reasonable when compared to experimental results. For a build height of 24.43 
$\mathrm{mm}$, the melt pool length observed through experiments was approximately $1.68 \mathrm{~mm}$, $1.55 \mathrm{~mm}, 1.45 \mathrm{~mm}$ and $1.25 \mathrm{~mm}$ respectively[12]. The melt pool depths for all the simulated cases were above $70 \mu \mathrm{m}$ which indicated that the previously deposited solid material is also melted ensuring proper interlayer bonding. The melt pool depth also decreased with increasing speed due to lowered maxima temperature.

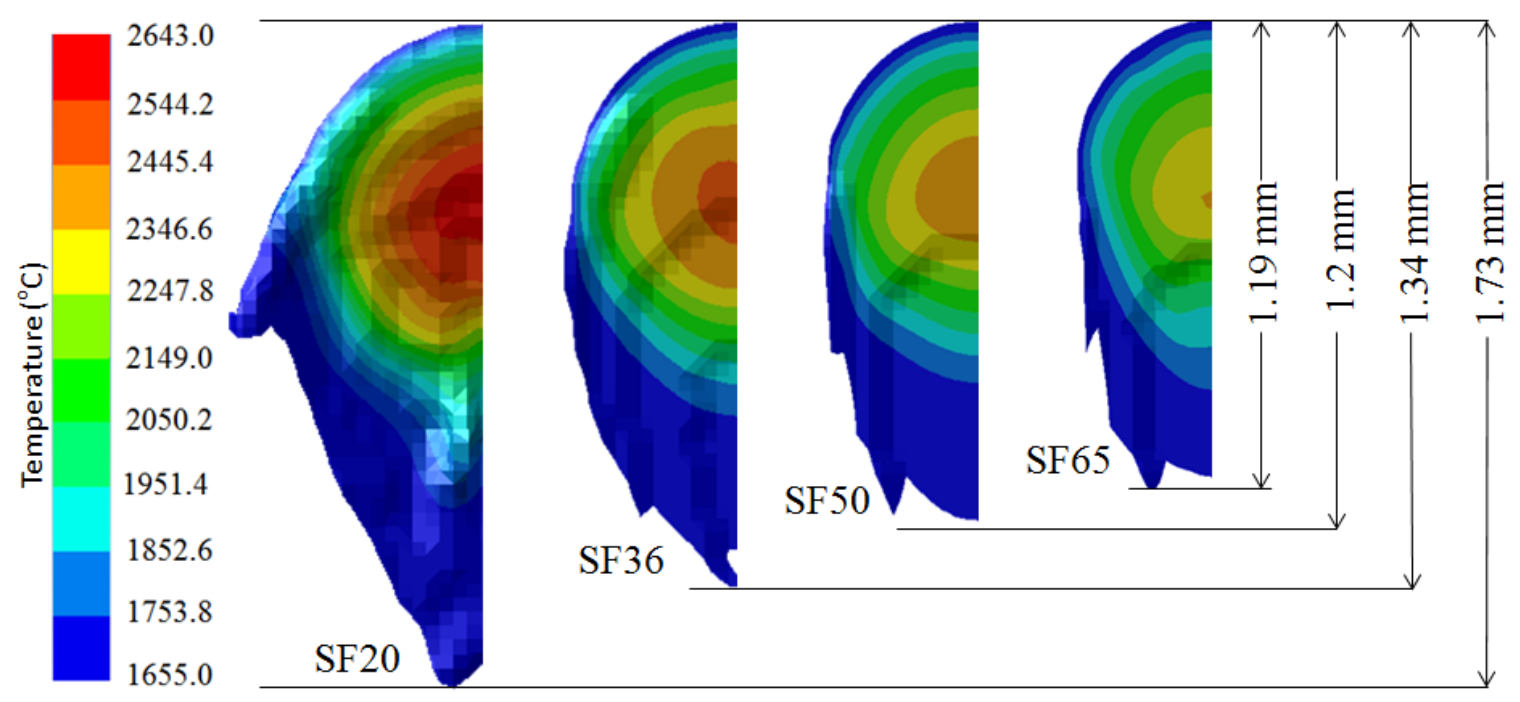

Figure 3.14 Melt pool shapes from free surface for different SF cases

\subsubsection{Velocity distribution}

The velocity profile in the transverse direction is summarized in Figure 3.15. The velocity at the center of the melt pool is comparatively lower and the maximum velocity is observed at the edges of the melt pool which is due to higher thermal gradient at the edge of the melt pool. This relatively higher velocity at the edge of melt pool leads to formation of humps at the outer region. We may also observe the change in velocity field with respect to the beam speed. The results show that the maximum velocity, obtained from first track, within melt pool for SF20 case is $0.36 \mathrm{~m} / \mathrm{s}$ whereas for SF50 and SF65 
cases are $0.2 \mathrm{~m} / \mathrm{s}$ and $0.16 \mathrm{~m} / \mathrm{s}$. Moreover, there is less melt flow for higher speed cases as the melt pool size decreases. Therefore, for very high beam speed, the melt flow may not be sufficient to form a uniform surface. In addition, the magnitude of hump formation depends upon the velocity component which is perpendicular to the scanning area. Figure 3.16 compares the maximum z-component of velocity which also decreases with increasing speed. Therefore, the hump formation in SF20 case may be dominant over higher SF cases.

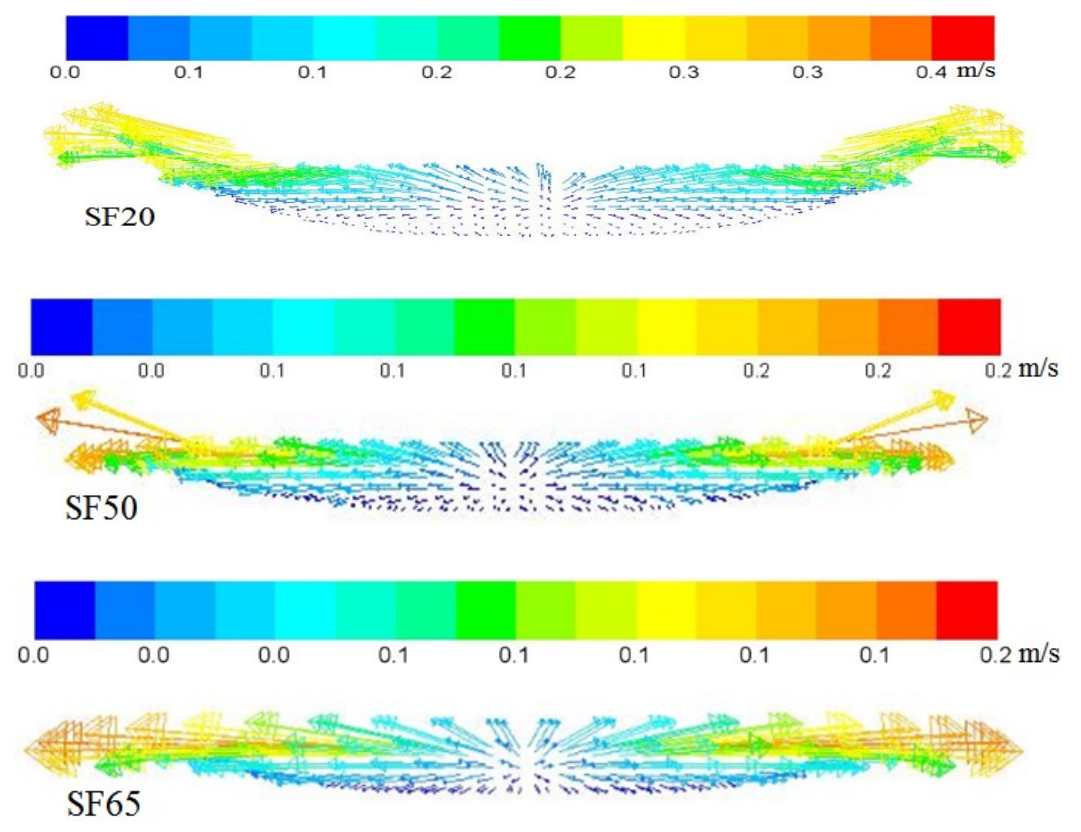

Figure 3.15 Velocity profile in the transverse direction for different SF cases. 


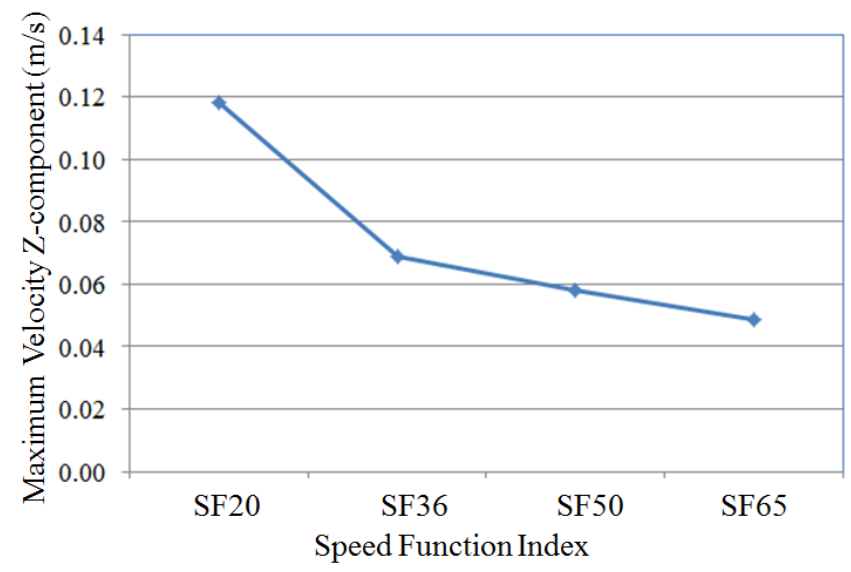

Figure 3.16 Maximum velocity in vertical direction for different SF cases

\subsubsection{Surface morphology}

The deformed surface after raster scanning is summarized in Figure 3.17. It is observed that the profile along the transverse direction shows similar trend in all the cases. As the beam speed increases, the highly deformed defective ball like structures are formed at different areas. For higher speed cases, due to decrease in intensity, the surface tension force is less dominant over the viscous drag. As a result, spreading of the molten pool may not be efficient which may result in higher surface roughness and formation of surface defects. We may see from the Figure 3.17(a) that for the same scanning region, due to widespread molten material in low speed case (SF20), the melted and solidified area is higher. The width of the melted and solidified region for SF20 case is around 2.32 $\mathrm{mm}$ whereas for SF50 and SF65 cases, it is around $2.07 \mathrm{~mm}$ and $1.99 \mathrm{~mm}$ respectively. Moreover, due to higher velocity magnitude, the hump formation is more dominant and at the end of the scan tracks, sharp elevated humps are formed for SF20 case. Although, we may observe comparatively disturbed surface formed after certain scanning in SF20 
case, there seems to be no surface defects in this case. For higher speed, the melt flow is not adequate which gives rise to highly deformed surface within the scan area.

In order to study the effect of hatch spacing on the surface formation, a SF65 case with hatch spacing $0.1 \mathrm{~mm}$ and $0.3 \mathrm{~mm}$ were simulated. The results indicate that increasing the hatch spacing decreases the stacking of the material towards the hump. SF65 case with hatch spacing $0.2 \mathrm{~mm}$ has higher material stacked towards the hump compared to $0.3 \mathrm{~mm}$ hatch spacing as shown in Figure 3.17(c). When the hatch spacing was reduced to $0.1 \mathrm{~mm}$, the material stacking increased which is pictured in Figure 3.17(e). Based on the formed surface, we may say that hatch spacing would affect the magnitude of surface roughness.

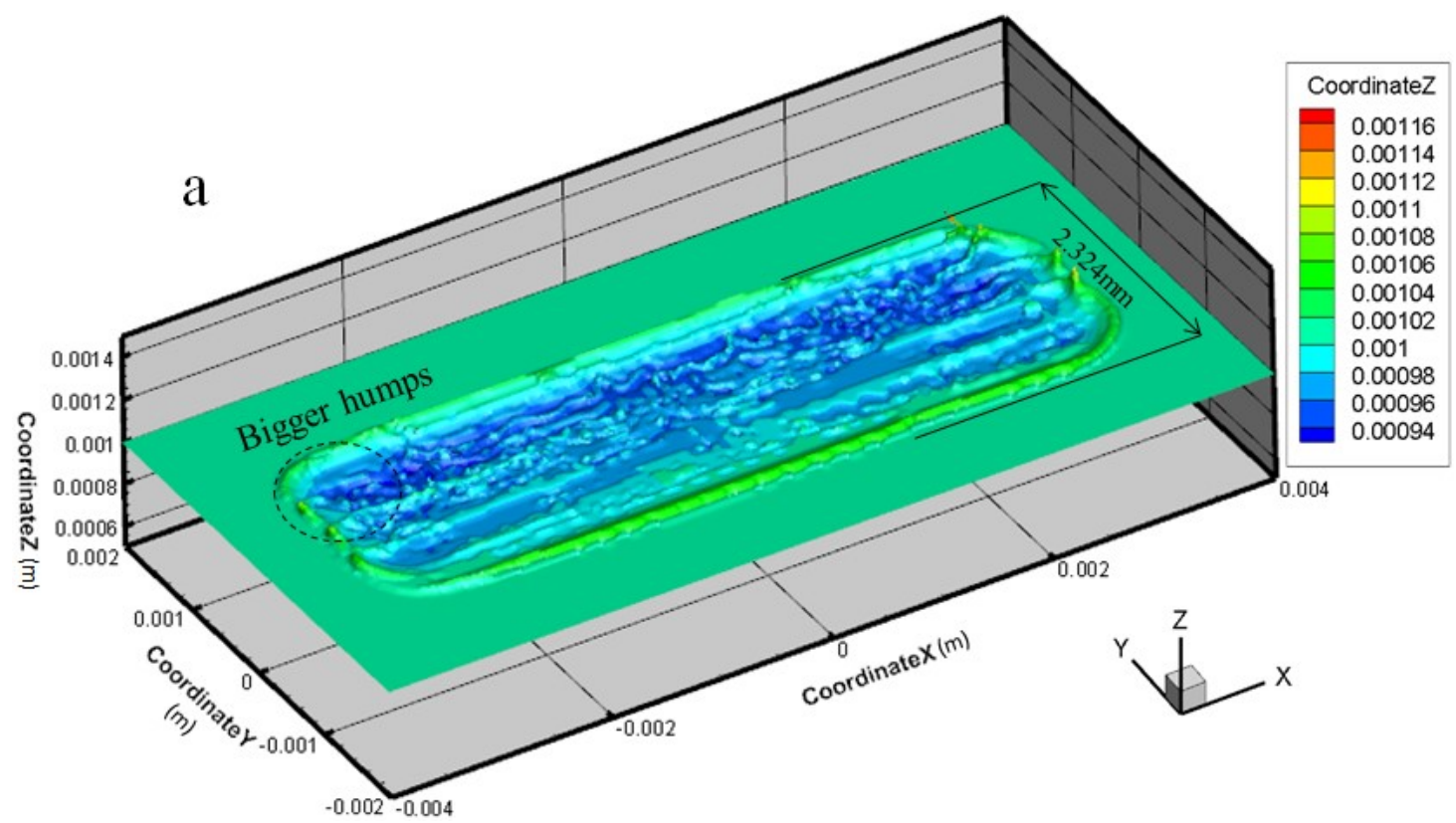



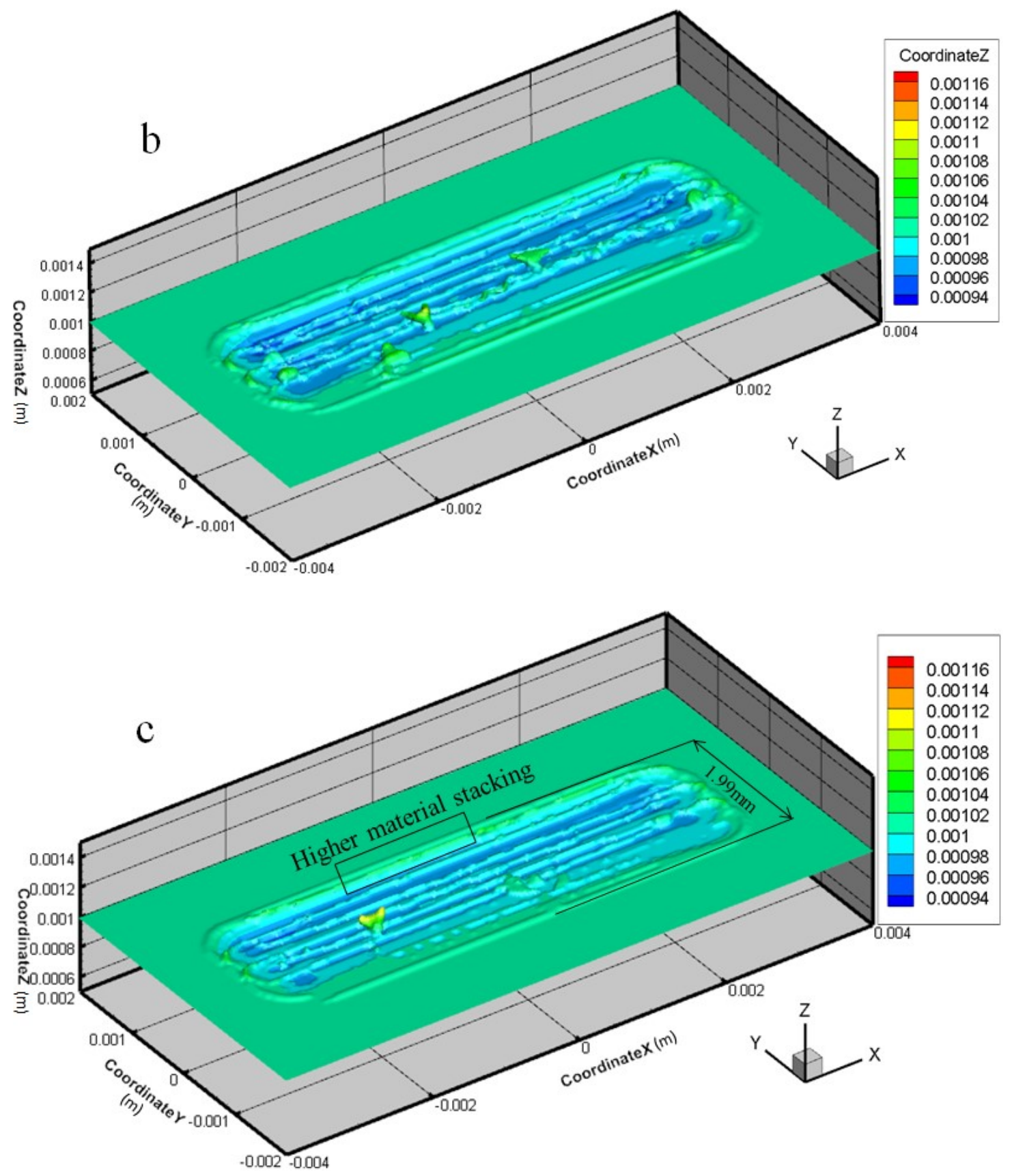

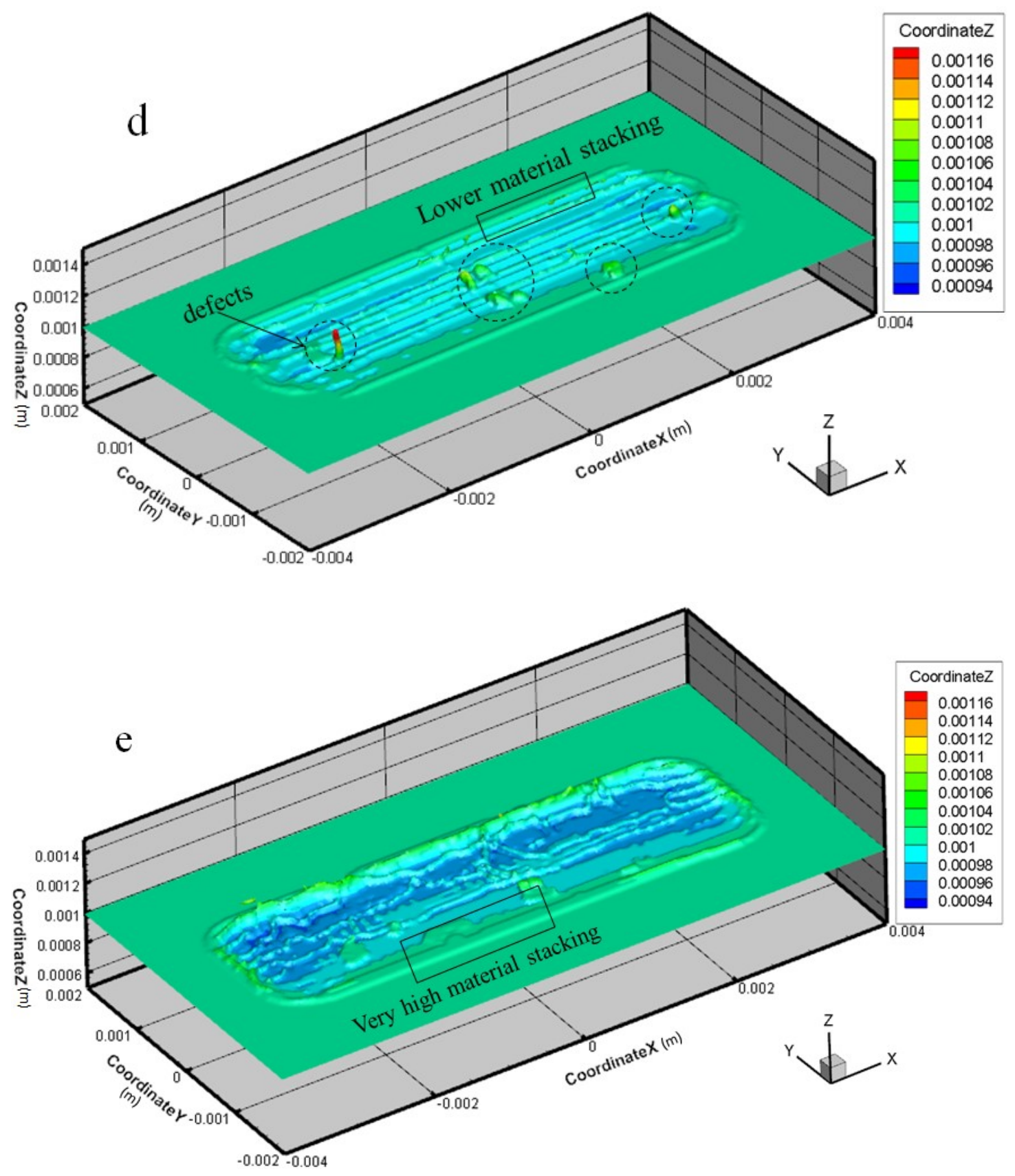

Figure 3.17 Surface morphologies of solidified top surface by simulation for (a) SF20, (b)SF50, (c) SF65, (d)SF65 with $\mathrm{H}=0.3 \mathrm{~mm}$ and (e) SF65 with $\mathrm{H}=0.1 \mathrm{~mm}$.

\subsubsection{Surface Roughness Measurement}

The deformed surface was obtained after raster scanning. Free surface data were extracted from a transverse line passing through the origin. The transverse surface profile 
was obtained and the calculated average roughness over a selected sampling length is summarized in Figure 3.18.
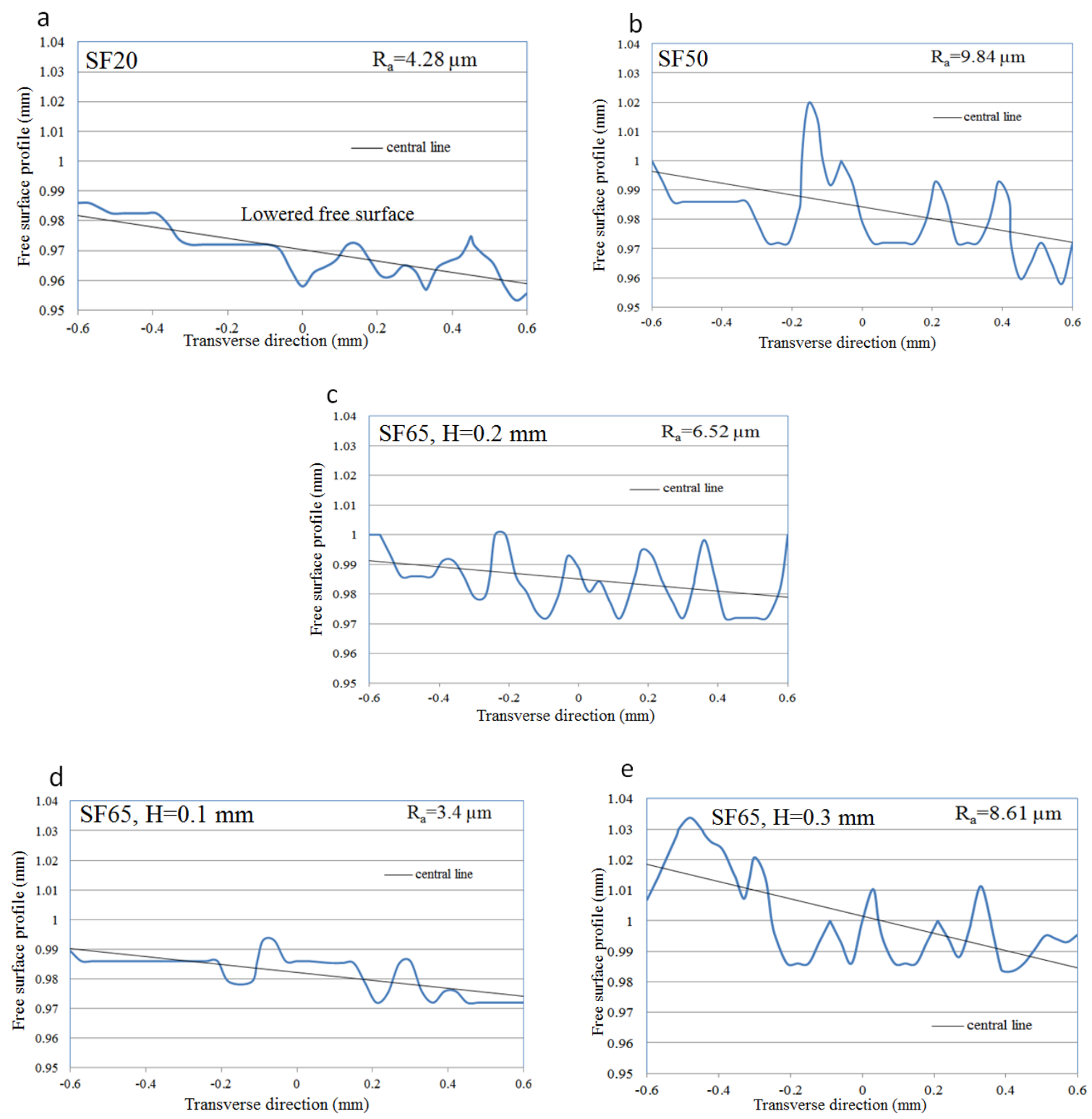

Figure 3.18 Surface roughness from simulation results along the transverse direction for different cases: (a) SF20, (b) SF50, (c) SF65, (d) SF65 with $\mathrm{H}=0.1 \mathrm{~mm}$ and (e) SF65 with $\mathrm{H}=0.3 \mathrm{~mm}$

The roughness analysis shows that the surface roughness increases with speed function and is lowest for SF20. For this low speed case, it is also observed that the average free surface height after deformation is lowest which indicate more shrinkage. 
This may be due to higher melt flow and adequate distribution of the melt in the surface over wider area. Although the Ra results obtained from simulation for SF20, SF36 and SF50 cases are comparable to experiment, the model predicted lower surface roughness for SF65 case. That is, the model was not able to predict highly porous surface formed for SF65 case which contributed for greater surface roughness value. When the beam speed is very high, the melt pool is smaller and there may not be adequate melt flow due to which pores may be formed. This phenomenon appears due to improper melting of powder particles which is not captured in this model developed under continuum approach.

The hatch spacing may also affect the surface roughness. Two different hatch spacing: $0.1 \mathrm{~mm}$ and $0.3 \mathrm{~mm}$ were used for SF65 case in addition to $0.2 \mathrm{~mm}$. It can be observed that, when the hatch spacing was reduced, the average surface roughness decreased, whereas when the hatch spacing was increased, the average roughness increased. The transverse profile for $\mathrm{H}=0.1 \mathrm{~mm}$ is rather flat compared to $0.2 \mathrm{~mm}$ and 0.3 $\mathrm{mm}$. Therefore, we may say that hatch spacing plays great role in surface roughness of PB-EBAM parts.

\subsection{Conclusions}

In this study a VOF model was applied to investigate the surface formation in the PB-EBAM process through finite volume approach. Temperature dependent material properties were defined for Ti-6Al-4V and volumetric heat source was applied by tracking the interface between melting powder and vacuum. Follows conclusions can be made from this study: 
1. The Marangoni shear force is function of temperature gradient. Therefore, for lower beam speed, Marangoni effect is dominant which results in wider melt region. The higher surface tension acts at the edge of the melt pool and tends to draw the molten metal towards the boundary of melt pool. As a result, humps are formed at the edges.

2. Beam speed affects the surface formation and roughness of the PB-EBAM parts. In general, the average roughness increases with speed function. Experiment results shows that SF65 case has higher surface roughness due to formation of pores, but for this particular case simulation predicted lower $R_{a}$ of $6.52 \mu \mathrm{m}$. However, for other cases, the $\mathrm{R}_{\mathrm{a}}$ values predicted by simulation are comparable to experimental results.

3. Surface roughness in transverse direction was found to be higher compared to scanning direction. Semi-periodic waves are observed which is the function of hatch spacing and it significantly contributes to the roughness in the transverse direction. Therefore, roughness can be reduced by using optimum hatch spacing.

4. The numerical model was able to capture various surface features of PB-EBAM process. The temperature melt pool size and roughness predicted by free surface simulation were in good agreement with experiment. 


\section{CHAPTER 4 THERMO-FLUID MODELING OF SELECTIVE LASER MELTING: SINGLE-TRACK FORMATION INCORPORATING METALLIC POWDER}

\subsection{Introduction}

Selective Laser Melting (SLM) is an additive manufacturing (AM) process which utilizes laser source to melt the powder layers. Computer aided design (CAD) data is decomposed into numerous layers based on the layer height and each layer is formed by multiple overlapping scan vectors. During each scan vector, as laser irradiates the powder particle, it melts the powder as well as previously deposited layers forming a melt pool within which the flow occurs. As the laser moves, the single track is formed due to solidification in the wake of the laser beam.

The property of single track would determine the final part quality. Each track is formed due to melting through heat transfer, fluid flow, and solidification and the track quality depend on the energy density applied. Hence the effect of varying energy density on the formation of single track needs to be investigated as the end parts of SLM process is highly dependent on the process parameters selected and it is always desired to optimize the process parameters. To achieve this, numerous experimental studies have 
been performed [58],[59]. Depending on the process parameters, the resultant single tracks may have different forms: continuous track with a crescent or elliptical section, discontinuous which may be irregularly broken or balled or only partially melted [60]. If the energy density is not enough, balling phenomenon is observed which occurs due to lower wetting ability and is dominant for lower laser power and higher scanning speed $[61,62]$. These single scan track experiments help narrow down the process parameter window that would result in dense part and good surface finish.

Single track experiment help distinguish appropriate process parameters that would result in dense single tracks without any balling or partial melting. However, thermo-fluid modeling would help understand the inherent fluid flow that leads to the formation of single track. Combined experimental and simulation study is desirable as the simulation help explain what is happening during experimentation which may otherwise be unknown. If the energy density is very high, evaporation occurs which result in keyhole formation and keyhole pores. If the energy density is very low, then discontinuous scan tracks are formed as a result of balling formation. These transitions can be studied through thermo-fluid simulations which would assist experimental study to understand and develop process window. However, SLM being powder bed process, powder particles need to be properly modeled as it would affect the heat transfer as well as fluid flow. Hence, several studies have been focused on mesoscopic modeling of SLM process. Khairallah and Anderson [24] developed a powder scale model which was able to predict the free surface formation while using constant surface tension. But, it is important to consider Marangoni effect and Lee and Zhang [25] developed a 3D thermofluid model using commercial Flow-3D software incorporating surface tension gradient. 
Back and forth two-track simulation was performed, and the simulation result has been compared with after two-track experiment. Similar modeling approach has also been applied to predict the effect of powder layer thickness on the single track formation [63]. Khairallah et. al. [64] performed high fidelity modeling including complex physical phenomenon and elaborated the mechanisms of pore formation, spatter and denudation zones. As the melt pool temperature often exceeds the boiling temperature, it is important to consider recoil pressure as well. Wu et al. [26] demonstrated the difference in melt pool depth prediction with and without using recoil pressure. If the recoil pressure is ignored, then the depth of the melt pool is underpredicted which is not desirable.

SLM is a layer upon layer process and therefore, the effect of additive layer towards the part formation should also be studied. This would be possible through mesoscopic model when a layer of powder is added to previously scanned layers. Panwisawas et al. [65] used a simplified powder scale model to understand the behavior of fluid flow towards the formation of pores due to the insufficient melting of powder particles. In addition, build surface formation as well as surface roughness can be predicted more accurately by using powder scale model. However, it would incur a high computational cost to perform area scanning with powder scale model; therefore, studies have been limited to continuum models. Dai and $\mathrm{Gu}$ [22] developed a two phase continuum model to predict build surface formation at different process parameters. In a similar manner, three phase model has also been developed for Powder Bed Electron Beam Additive Manufacturing (PB-EBAM) process [66].

In this study, a sequential addition algorithm was used to obtain the powder distribution over the base plate. A 3D volume of fluid (VOF) model was developed to 
predict the heat transfer, fluid flow and resulting track morphology during single track formation. The transport phenomenon in the melt pool is investigated to understand the formation of a single track during SLM process. In addition, a layer addition algorithm is developed, and multi-layer simulation is performed.

\subsection{Numerical Modeling}

\subsubsection{Powder scale model}

A 3D model was developed with domain size $1200 \mu \mathrm{m} \times 400 \mu \mathrm{m} \times 250 \mu \mathrm{m}$ as shown in figure 4.1. Powder particles are defined by using sequential powder addition algorithm[67] with some modifications to the reported formulas (equations 4.1-4.3). When a powder particle drops, there may occur four situations: (a) a falling sphere rolls over previously settled sphere, (b) falling sphere rolls over two previously settled spheres, (c) falling sphere becomes stable after being in contact with three spheres, and (d) sphere is not stable even if in contact with three sphere and has to roll further down to stabilize. The algorithm has been discussed in detail in the literature [67] and only case (a) has been shown in Figure 4.1 due to the modification made to the powder position update formula. Point B in Figure 4.1 represents the contact point between sphere 1 and 2, point $\mathrm{O}$ is the center of sphere 1 which is the reference for the coordinate system. Equation 4.14.3 represent the change in position of sphere $1\left(\mathrm{x}_{1}, \mathrm{y}_{1}, \mathrm{z}_{1}\right)$ while rolling down sphere 2 $\left(\mathrm{x}_{2}, \mathrm{y}_{2}, \mathrm{z}_{2}\right)$ due to the change in zenith angle $\theta$ represented by $\delta \theta$. 


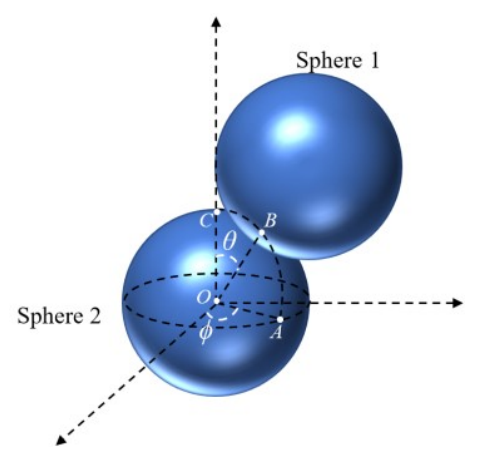

Figure 4.1 Incoming sphere rolling down over the previously deposited sphere.

$$
\begin{aligned}
& \mathrm{x}_{1}=\mathrm{x}_{2}+\left(\mathrm{r}_{1}+\mathrm{r}_{2}\right) \cdot \sin (\theta+\delta \theta) \cdot \sin \phi \\
& \mathrm{y}_{1}=\mathrm{y}_{2}+\left(\mathrm{r}_{1}+\mathrm{r}_{2}\right) \cdot \sin (\theta+\delta \theta) \cdot \cos \phi \\
& \mathrm{z}_{1}=\mathrm{z}_{2}+\left(\mathrm{r}_{1}+\mathrm{r}_{2}\right) \cdot \cos (\theta+\delta \theta)
\end{aligned}
$$

where $r_{1}$ and $r_{2}$ are the radius of sphere 1 and 2 respectively and $\phi$ is the azimuth angle.

Above mentioned algorithm has been applied to obtain random powder distribution which would represent the powder bed condition. In this study, powder size distribution from $15 \mu \mathrm{m}-40 \mu \mathrm{m}$ has been considered and the particles are defined over the $90 \mu \mathrm{m}$ solid Ti-6Al-4V substrate as shown in Figure 4.2. Volume fraction in Figure 4.2 differentiates two materials: Ti-6Al-4V and argon. Ti-6Al-4V is defined by volume fraction 1 while argon has volume fraction zero and the free surface has volume fraction in between 0 and 1 and therefore represented by green color. To properly capture the volume fraction, a hexahedral mesh is defined over the domain. Mesh size of $5 \mu \mathrm{m}$ has been used. The powder particles generated by sequential addition algorithm was read through FLUENT user-defined function (UDF) 


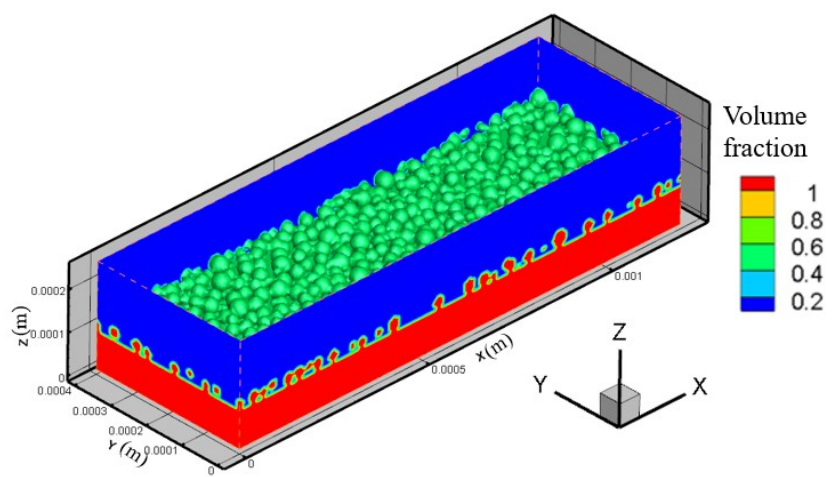

Figure 4.2 Computational domain with powder particles distribution used for simulation

\subsubsection{Governing equations}

Laser heat source has been modeled as the conical volumetric heat source which is represented in Eqn 4.4 .

$$
\dot{Q}_{(x, y, z)}=\eta \times \frac{H_{S} \times I_{z}}{S}
$$

where,

$$
\begin{gathered}
I_{z}=\frac{1}{0.75}\left(-2.25\left(\frac{z}{S}\right)^{2}+1.5\left(\frac{z}{S}\right)+0.75\right), \\
H_{S}=\frac{2 P}{\pi d^{2}} \exp \left\{-\frac{2\left[\left(x-x_{S}\right)^{2}+\left(y-y_{S}\right)^{2}\right]}{d^{2}}\right\} .
\end{gathered}
$$

where $\eta$ represent absorption efficiency, $\mathrm{P}$ is laser power, $\mathrm{S}$ represent penetration depth, $\mathrm{d}$ is beam diameter, $\mathrm{x}_{\mathrm{S}}$ and $\mathrm{y}_{\mathrm{S}}$ are heat source center position. Hs and $\mathrm{I}_{\mathrm{z}}$ are horizontal Gaussian distribution heat source and heat source magnitude decaying function in the vertical direction, respectively. The heat source is defined in the UDF and applied as the source term in the energy equation. The laser source is moved by updating $\mathrm{XS}_{\mathrm{S}}$ and ys position based on the scanning speed. 


\subsubsection{Tracking of free surface}

The VOF equation which is used to track the free surface during melting and solidification is given as

$$
\frac{\partial F}{\partial t}+\vec{v} \cdot \nabla F=0
$$

where, $F$ represents the volume fraction and $\vec{v}$ represents the velocity vector. In this model, two phases have been used: argon as phase 1 with volume fraction 0 and Ti$6 \mathrm{Al}-4 \mathrm{~V}$ as phase 2 with volume fraction 1 . A free surface of the powder which interacts with the argon has the volume fraction between 0 and 1 . VOF equation is solved along with mass, momentum and energy equations.

\subsubsection{Boundary conditions}

The boundary condition at the interface is given as

$$
\frac{\partial(\rho H)}{\partial t}+\frac{\partial\left(\rho u_{i} H\right)}{\partial x_{i}}=\frac{\partial}{\partial x_{i}}\left(k \frac{\partial T}{\partial x_{i}}\right)+\dot{Q}(x, y, z)-h A\left(T-T_{\infty}\right)-\sigma A \varepsilon\left(T^{4}-T_{\infty}^{4}\right)
$$

where, $\mathrm{H}$ is the enthalpy, $\mathrm{k}$ is thermal conductivity, $\dot{Q}_{(x, y, z) \text { int }}$ is the heat source applied at the interface, $\mathrm{h}$ is the heat transfer coefficient, $\sigma$ is the Stefan-Boltzmann constant and $\varepsilon$ is the emissivity which is used to account for the radiation through the top surface and $\mathrm{A}$ is the free surface area of cell. Besides the free surface, all the walls in the domain have adiabatic boundary conditions. 


\subsubsection{Application of volumetric heat source}

In the mesh-based simulation, the free surface should be properly identified to appropriately apply the volumetric heat source which is of even more importance due to spherical powder particles. In addition, the surface is continuously deforming during the melting process. Therefore, the topmost cell with non-zero phase- 2 volume fraction which is the actual free surface must be tracked after each iteration which is achieved through UDF. Figure 4.3 demonstrates how the topmost cell for each column is identified. Volume fraction for each cell in a column is read and the $\mathrm{z}$-coordinate of each cell, from the same column with volume fraction in the interval $(0,1)$ is compared. Finally, the topmost cell which is irradiated to the laser is defined as free surface for that column. The arrowhead points to the free surface cell which is pointed for each cell beneath the topmost cell. The free surface cell information is utilized to define the magnitude of heat source for cells beneath based on the penetration depth.

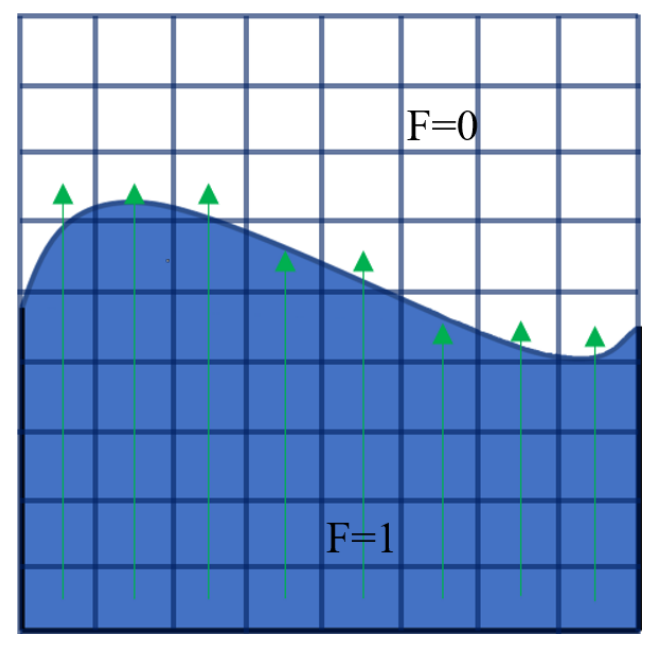

Figure 4.32D representation of topmost free surface identification to apply a volumetric heat source 


\subsubsection{Material Properties}

Physical properties such as thermal conductivity, specific heat, density are the function of temperature. Therefore, temperature dependent material properties summarized in Figure 4.4 are used to define solid and powder Ti-6Al-4V. As the properties are almost linear with the temperature, they are defined as piece-wise linear in Fluent. Besides these, surface tension is also the function of temperature. Additional material properties of Ti-6Al-4V have been summarized in table 4.1. Generally, the surface tension of metal can be defined by equation 4.7 .

$$
\gamma=\gamma_{m}+\frac{d \gamma}{d T} \Delta T
$$

where $\gamma$ is the surface tension, $\gamma_{m}$ is the surface tension at the melting point, $\frac{d \gamma}{d T}$ is the surface tension gradient and $\Delta T$ is the temperature difference in reference to the melting temperature.
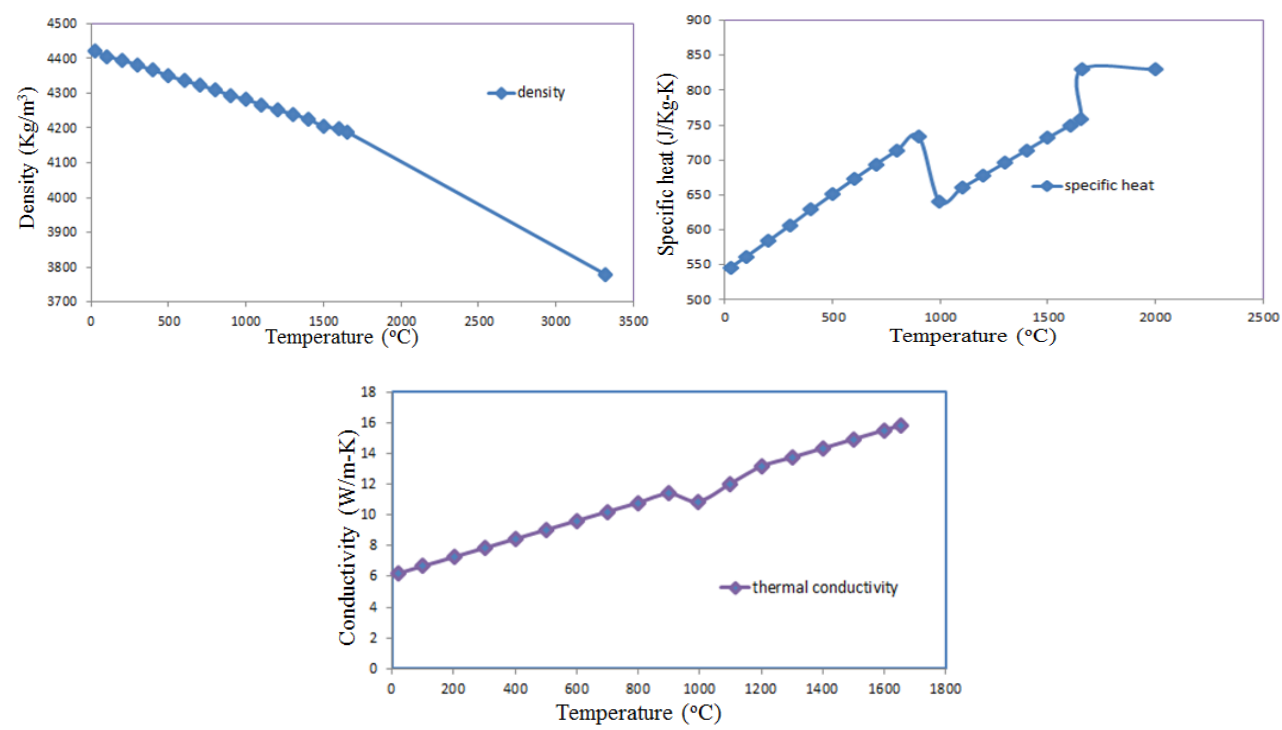

Figure 4.4 Temperature dependent material properties of Inconel Ti-6Al-4V [13]. 
Table 4.1 Material properties of Ti-6Al-4V

\begin{tabular}{ll}
\hline Parameters & Values \\
\hline Solidus temperature, $T_{S}\left({ }^{\circ} \mathrm{C}\right)$ & $1605[51]$ \\
Liquidus temperature, $T_{L}\left({ }^{\circ} \mathrm{C}\right)$ & $1655[51]$ \\
Latent heat of fusion, $L_{f}(\mathrm{~kJ} / \mathrm{Kg})$ & $440[51]$ \\
Beam diameter, $\mathrm{d}(\mu \mathrm{m})$ & 100 \\
Power $(\mathrm{W})$ & 195 \\
Viscosity $(\mathrm{kg} / \mathrm{m}-\mathrm{s})$ & 0.049 \\
Emissivity, $\varepsilon$ & $0.5[68]$ \\
Stefan-Boltzmann constant, $\sigma$ & $5.67 \times 10^{-8}$ \\
\hline
\end{tabular}

\subsection{Results and Discussion}

\subsubsection{Single layer simulation}

\section{Typical simulation}

The result of melt pool flow for scanning speed $600 \mathrm{~mm} / \mathrm{s}$ and power $195 \mathrm{~W}$ is described in this section. When the heat source is not applied, powder particles remain solid. The application of heat source increases the temperature of a certain volume of powder with the highest temperature at the center of the laser beam. With further increase in the temperature, powder particle at the center starts to melt which is then followed by the melting of surrounding powder particles as seen in Figure 4.5(a). The smaller powder particles would melt before larger powder particles in the powder particles mixture of 15 to $40 \mu \mathrm{m}$. The melted powder fills the void between powder particles due to the continuous flow of liquid and the melt pool becomes dense. The melt pool solidifies with the progress in time as the heat source moves from left to right. This process of melting and solidification continues to form a single track. The smaller powder will melt rapidly in case of high energy density and the temperature may exceed the boiling point of the material. This would result in evaporation and result in the higher depression below the 
heat source. In this calculation, the evaporation is not considered, and the effect of recoil pressure is also not included.

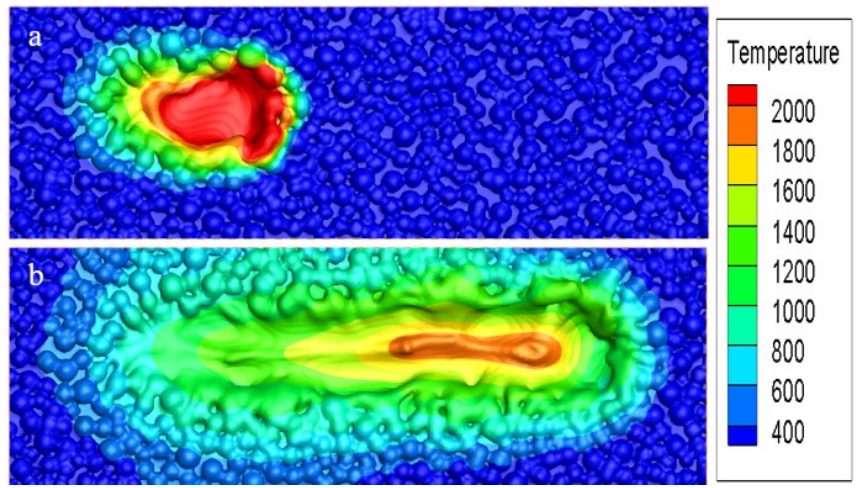

Figure 4.5 Top view of the powder bed showing (a) the melt pool at $\mathrm{t}=400 \mu \mathrm{s}$ and (b) solidified track at $\mathrm{t}=1600 \mu$ s along with the temperature distribution $\left({ }^{\circ} \mathrm{C}\right)$

During melting, the flow is outwards, from higher temperature to lower temperature due to surface tension gradient. The heat source is moved from the left to right which allows the rear to cool down due to heat diffusion. During the solidification, the melt flow changes direction from outer flow to inner flow which contributes to the formation of a continuous bead. Figure 4.6 illustrates the transient behavior of melt flow during single track formation. The transverse cross-section at $405 \mu \mathrm{m}$ for scanning speed $600 \mathrm{~mm} / \mathrm{s}$ with time progression has been presented in Figure 4.6. These figures represent the volume of fluid fraction. The spherical powder slowly starts to change its shape at $\mathbf{t}=$ 300s $\mu$ s. The melt pool depth keeps increasing up to $t=500 \mu$ s after which the solidification starts. During the solidification, the melt flow is pulled in the upward direction to form a track as seen in $\mathrm{t}=900 \mu$ s. The powder has been completely melted and the melt pool depth increases It clearly shows that the melt pool increases in depth 
during the heating period and then the flow reverse back under the effect of increasing surface tension to form a solid track.
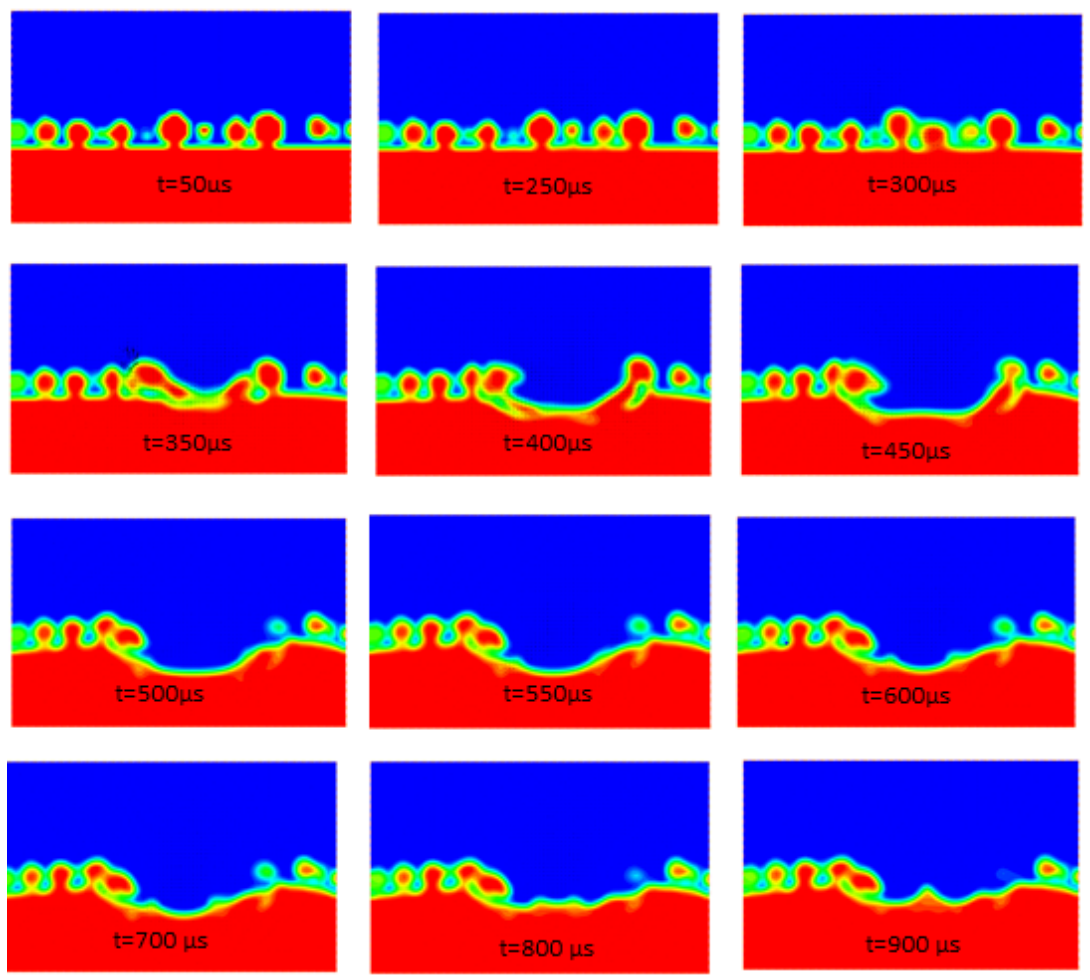

Figure 4.6 Melt pool dynamics during melting and solidification

In addition, it is observed that there is a shallow area at the end of the scan track. This may be due to the insufficient flow of melt pool at the end of the scan track. When the laser is turned off, the melt pool immediately solidifies leaving a shallow region at the end of the scan track. As the void region is filled during the solidification, the insufficient melt flow at the beam turn off point may result in pore formation.

A quick view just after the solidification at the point where the laser is stopped shows that the depth of that shallow region changes according to the scanning speed. 


\section{Process parameter effect}

Power and Speed affect the amount of energy being fed into the powder bed which ultimately affect the thermal response as well as melt flow. Therefore, melt pool formed due to change in scanning speed at same power of $195 \mathrm{~W}$ is has been summarized in Figure 4.7. We may observe that the melt pool width decreased with increasing speed. This is due to the temperature distribution within the melt pool, low speed result in higher temperature zones within the melt pool compared to high speed. The melt pool width observed during the simulation with $400 \mathrm{~mm} / \mathrm{s}, 600 \mathrm{~mm} / \mathrm{s}, 800 \mathrm{~mm} / \mathrm{s}$ and $1000 \mathrm{~mm} / \mathrm{s}$ were $220 \mu \mathrm{m}, 200 \mu \mathrm{m}, 160 \mu \mathrm{m}$ and $140 \mu \mathrm{m}$ respectively.
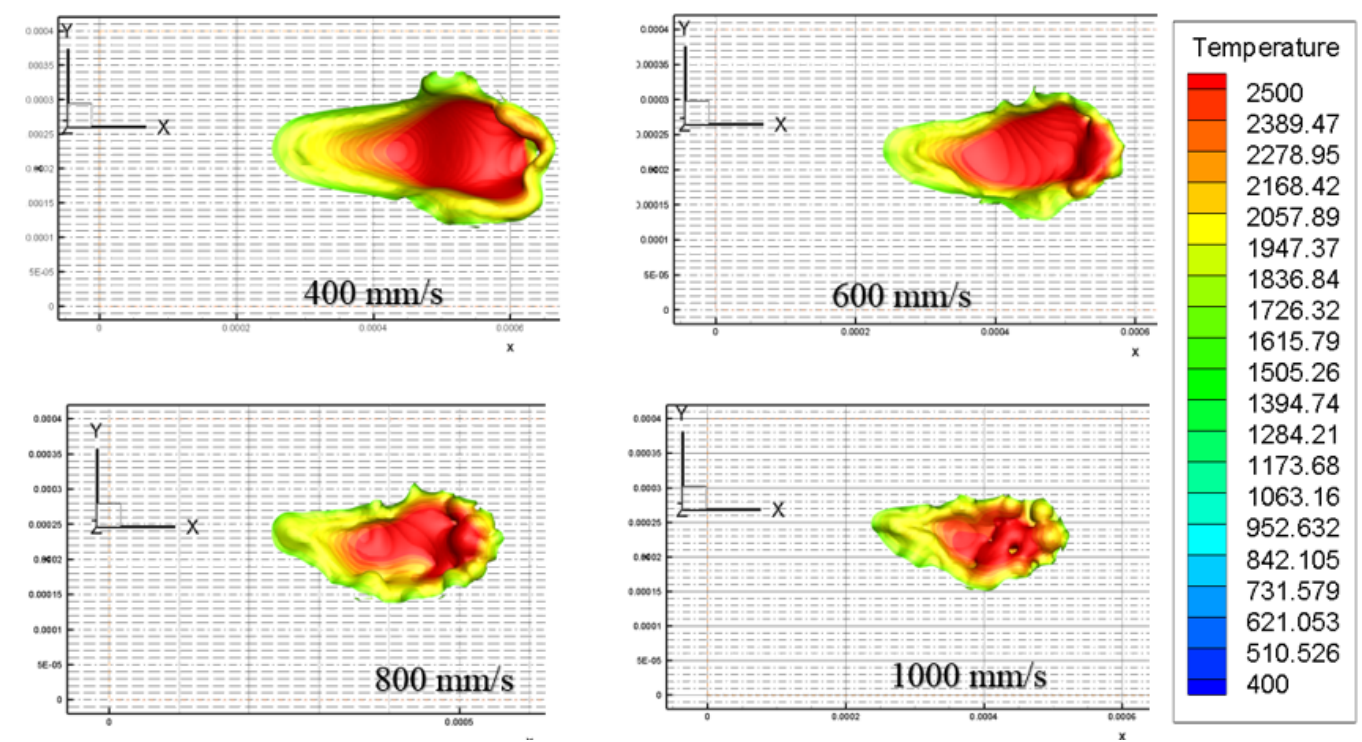

Figure 4.7 Melt pool comparison between different process parameters

Single track experiment was carried out in an EOS M270 machine with TiAl6V4 powder. Four single tracks were formed on the top surface with different scanning speeds $400 \mathrm{~mm} / \mathrm{s}, 600 \mathrm{~mm} / \mathrm{s}, 800 \mathrm{~mm} / \mathrm{s}$ and $1000 \mathrm{~mm} / \mathrm{s}$ at $195 \mathrm{~W}$ laser power. Then formed track surface was analyzed through the white light interferometer to obtain the melt pool 
width which has been depicted in Figure 4.8. The 2D cross section has been presented for two cases: $400 \mathrm{~mm} / \mathrm{s}$ and $600 \mathrm{~mm} / \mathrm{s}$. The bead height measured is above the layer thickness. At certain cross section, single track bead height for $400 \mathrm{~mm} / \mathrm{s}$ was $90 \mu \mathrm{m}$ whereas for $600 \mathrm{~mm} / \mathrm{s}$ was $80 \mu \mathrm{m}$ although $30 \mu \mathrm{m}$ was used during the experiment. Five similar cross sections were taken in a span of $1000 \mu \mathrm{m}$ from the interferometer and averaged to calculate the track width. Single data was taken from the computational result using Tecplot with an accuracy of $10 \mu \mathrm{m}$. The track width value obtained from the experiment follows the trend and is in good agreement with the computational result as shown in Figure 4.9.
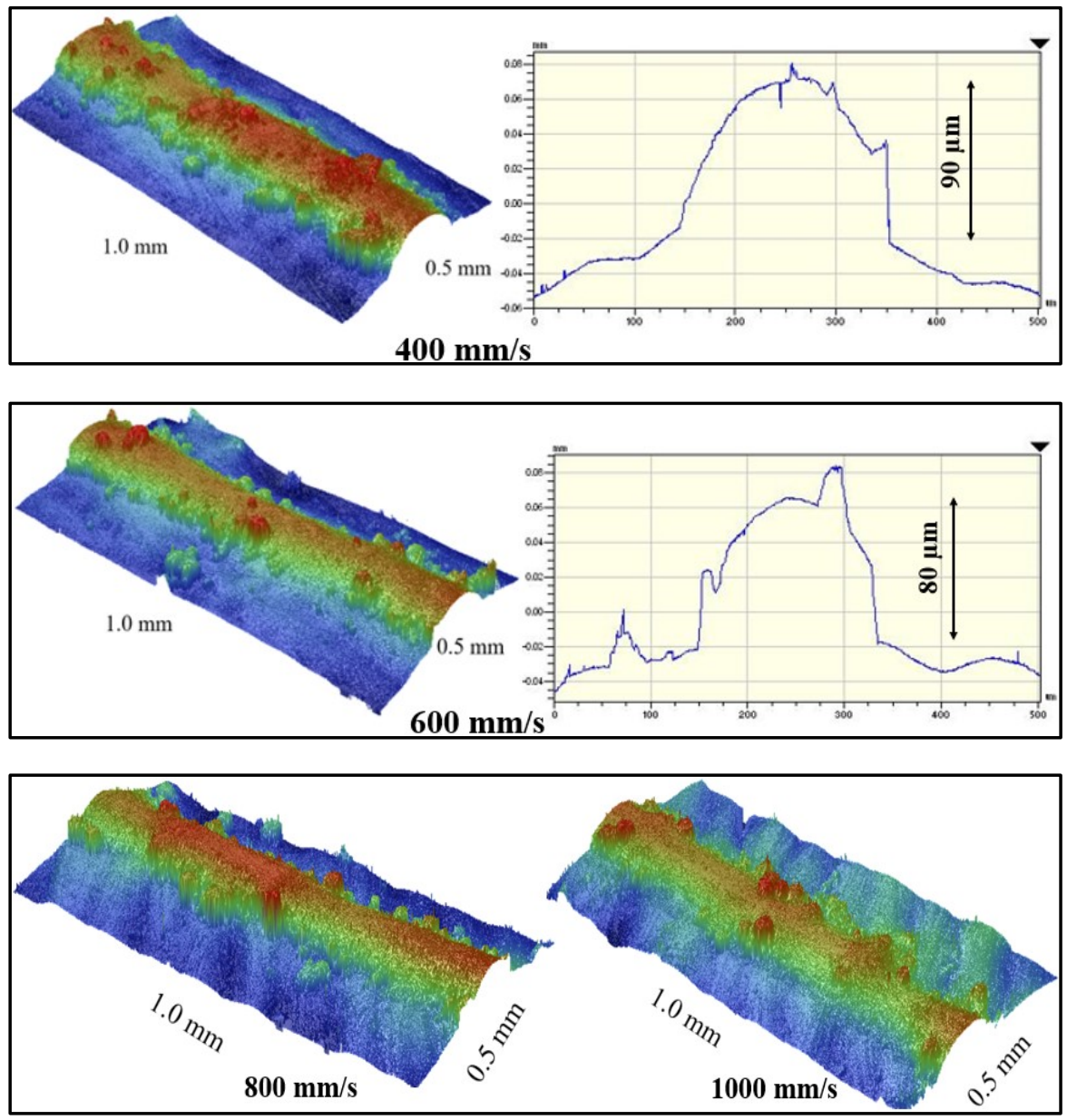

Figure 4.8 Surface morphology for four velocity values at a constant power of $195 \mathrm{~W}$ 


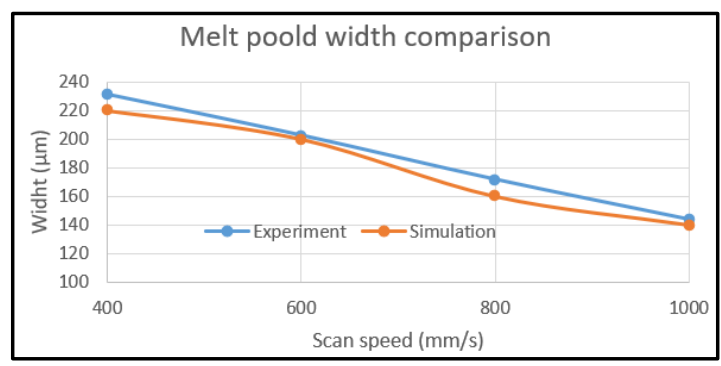

Figure 4.9 Melt pool width comparison between experiment and simulation

Figure 4.10 shows the single-track morphology predicted for different scanning speed. It is important to note that the single-track morphology is very different considered to the experimental scan tracks. There are several reasons for this difference. During the single track experiment, the neighbor powder particles get sucked into the melt pool which would add the mass into the single track, therefore increasing the bead height [69]. In addition, the layer thickness after certain height may be more than defined layer thickness to compensate for the shrinkage due to low powder packing density. In this simulation, these factors have not been considered and therefore very small bead is formed during solidification.
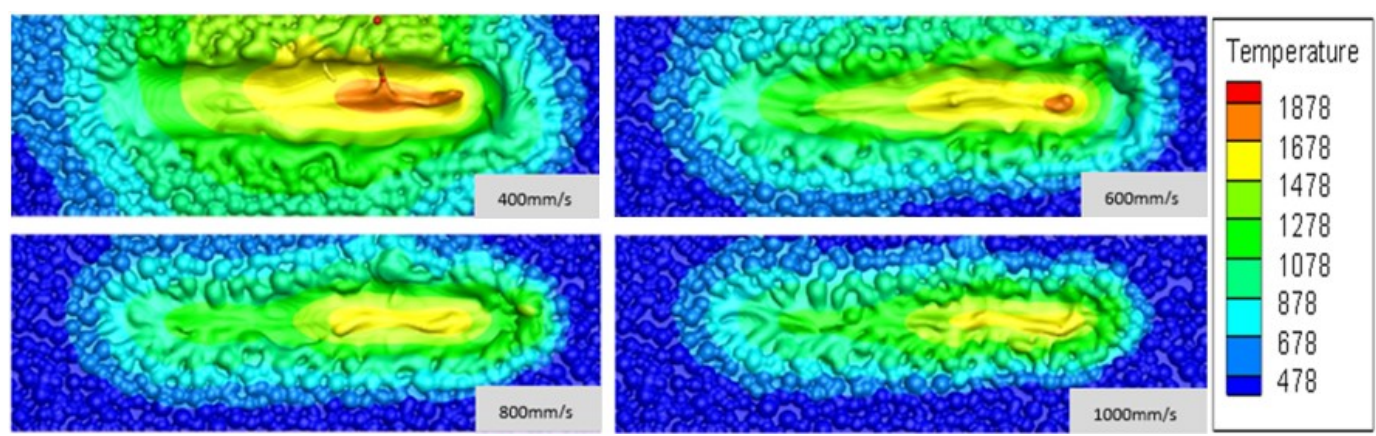

Figure 4.10 Solidified single tracks at different scanning speeds.

In the SLM process, laser turn off point is often important as there is often insufficient melt flow due to which pores may occur. Figure 4.11 help explain the track 
end morphology at different scanning speed. At low speed of $400 \mathrm{~mm} / \mathrm{s}$, there may be numerous pores formed at the end of the single track.
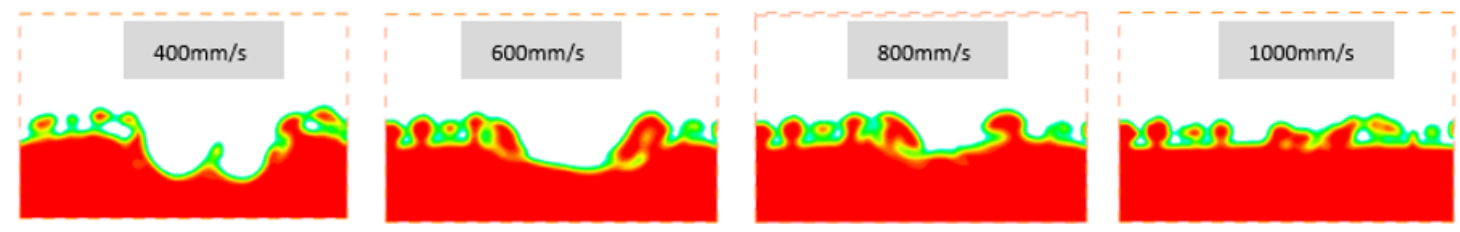

Figure 4.11 Image slice of the 3D domain at the shallow region for four scanning speed

\subsubsection{Two-layer simulation}

SLM is a layer by layer process and therefore the part quality depends upon the interaction between layers as well. In this prospect, multilayer simulation becomes important. A solidified track from the previous layer would affect the powder particle distribution. Therefore, a two-layer simulation is carried out. To achieve this, at first, onelayer simulation is performed and after its completion, the free surface, that is single track and neighbor powder particles are exported as a mesh. A program which was developed based on sequential powder addition algorithm was modified to read the free surface data. Figure 4.12 shows how the second layer algorithm works: the powder is dropped from a certain height and as soon as it comes in contact with the sphere, the stability is checked by reading the neighbor mesh. If the particle is not stable, then the iteration is continued and the particles are rolled down to lower mesh until the particle becomes stable. Moreover, if the incoming sphere comes in contact with a previously deposited sphere, the same algorithm which has been discussed earlier is followed. 


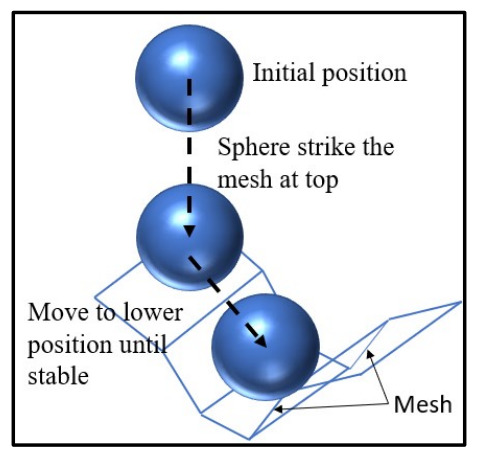

Figure 4.12 Second layer powder addition demonstration.

Thus developed second layer powder addition algorithm was applied to obtain the second layer of powder distributed over the first layer. Figure 4.13 illustrates the powder being added. We can clearly see from Figure 4.13(a) how the first layer is being covered up by second layer and after the complete addition, the first layer track is not visible.
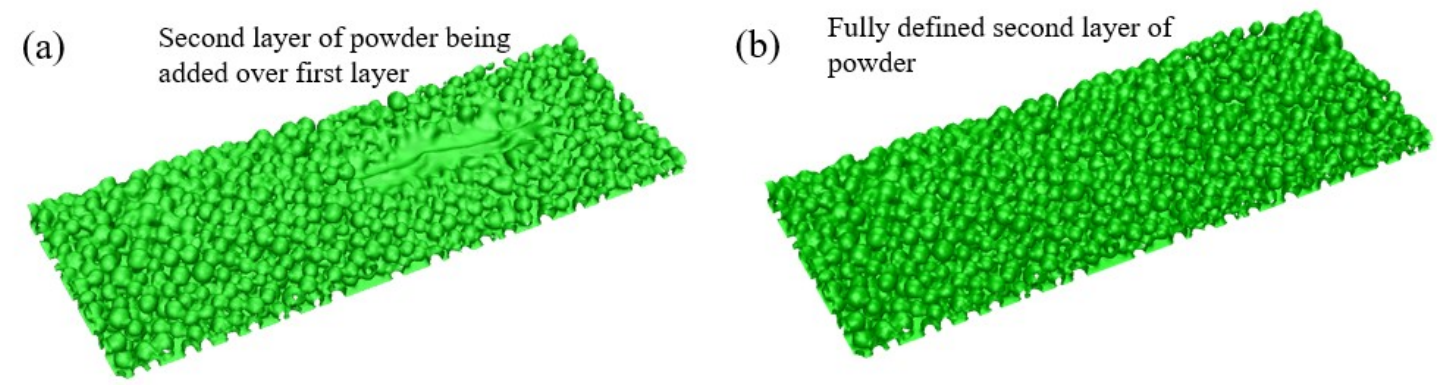

Figure 4.13 (a) Second layer of powder being defined over first layer and (b) second layer of powder after fully defined.

For two-layer simulation, higher scanning speed was selected to reduce the computational time, at first, $1000 \mathrm{~mm} / \mathrm{s}$ scanning speed is used with $195 \mathrm{~W}$ power. Figure 4.14 shows the transient behavior of the free surface during the melting process. It has been experimentally shown that during the scanning process, the melt pool attracts neighbor particles[69]. We may observe similar phenomenon during the simulation as well. Initially, when heat is not applied, second layer powder particles are settled over the 
first layer. However, as the melt pool begins to form, its flow drives in such a manner that the neighbor particles get attracted to the melt pool as well. However, due to the meshbased simulation, the particles do not get mixed into the melt pool. Due to this limitation, bead formation would not be properly formed in this study.
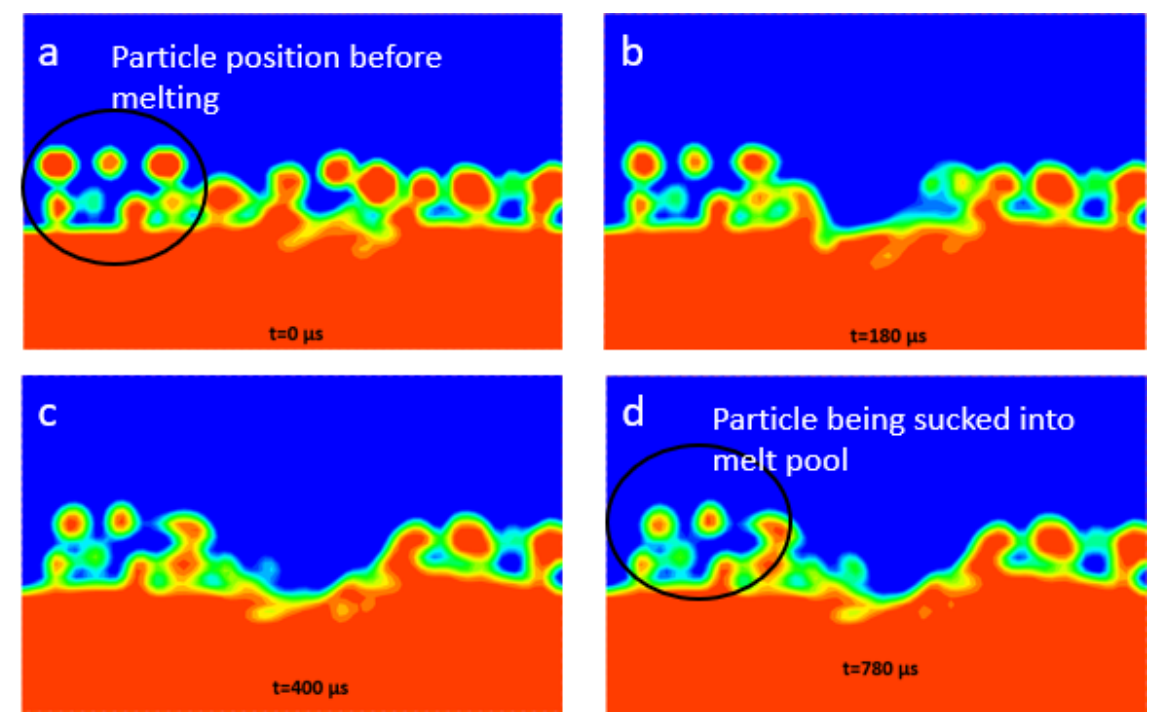

Figure 4.14 (a) Solidified single track after second layer scanning, (b) Transverse and (c) longitudinal cross section of solidified two layer-single track $(\mathrm{v}=1000 \mathrm{~mm} / \mathrm{s})$

\subsection{Conclusions}

In this study, sequential addition algorithm was adopted to obtain powder bed distribution over a solid substrate. A 3D thermo-fluid model was developed, and powder particles distribution was imported to study fluid flow during melting and solidification processes. Single-track formation in both single-layer and two-layer depositions were developed to investigate the fluid dynamics in the melt pool and the formation of a single track. Different scanning speeds were tested to study the temperature distribution, fluid velocity and the formation of pores. Based on the simulation results, following conclusions can be made. 
- Inside the metal molten pool, the outward flow is observed during the melting process, while the inward flow is observed during the solidifying stage.

- In the testing range, the track width decreases with decreasing the laser scan energy density.

- The single-track bead height from simulations is lower than that observed in the SLM experiments, which is even higher than the layer thickness due to possibly the denudation phenomenon.

- The free surface information from the first layer single track simulations can be used to obtain the second layer powder distribution. Proper interlayer bonding has been predicted for $195 \mathrm{~W}$ laser power and $1000 \mathrm{~mm} / \mathrm{s}$ scanning speed. 


\section{CHAPTER 5 A STUDY OF KEYHOLE POROSITY IN SELECTIVE LASER MELTING: SINGLE TRACK SCANNING WITH MICRO-CT ANALYSIS}

\subsection{Introduction}

Selective laser melting (SLM) is a metal-based additive manufacturing process which utilizes a laser to selectively melt powder particles and form the desired product after a layer-by-layer fabrication process [58]. SLM process has been applied to fabricate parts from different metallic materials such as aluminium [70, 71], nickel [72, 73], titanium $[4,74]$, steel $[31,75]$, etc. Among those, Titanium alloy Ti-6Al-4V has been favored in aerospace industries [32] as well as biomedical applications [76-78] due to its high specific strength and biocompatibility [79].

In spite of its freeform capability, the SLM process has issues of process-induced defects such as porosity, cracks, part deformation, etc $[80,81]$. Pores formed during the process may be characterized as the keyhole pores and the lack of fusion pores [5]. These pores if present within the SLM parts would degrade its part performances [31-33] and therefore part optimization by porosity reduction is desired. Leuders et al. [33] explained that pores within SLM samples have a drastic effect on fatigue behavior and the significant extension of the crack initiation phase can be achieved by reducing the porosity. 
Pore formation during the SLM process is affected by the energy density input which is a function of the laser power, the scan speed, the hatch spacing, and the layer thickness. Gong et al. [35] investigated part quality based on different energy densities and demonstrated that the process window in SLM of Ti-6Al-4V could be divided into three groups, insufficient melting, conduction melting, and keyhole melting. When the energy density is very high, the temperature within the melt pool exceeds the boiling point leading to material evaporation. The material evaporation would generate an evaporation pressure, and the deep depression formed within the melt pool is known as a keyhole. Such melting is known as keyhole-mode melting [36]. As the energy density gets reduced, keyhole formation is not observed, and the mode of melting is called the conduction melting. If the energy density gets further reduced, partial melting occurs which lead to balling behavior and discontinuity in the track formation.

A few investigations towards part porosity developed during the SLM process have been carried out. Ponnusamy et al. [6] performed a statistical analysis to investigate the effect of various process parameters such as laser power, layer thickness, etc. towards porosity. Besides, various studies have been conducted to characterize porosity using Xray tomography methods. Slotwinski et al. [7] utilized X-ray computed tomography (XRCT) to measure porosity on additively manufactured cobalt-chrome (Co-Cr) samples. The XRCT technique can help determine the pore distribution within the sample as well as the pore morphology. However, the accuracy of the representation would depend on the resolution of the system. Similarly, Zhou et al. [37] used Synchrotron radiation microCT to observe the defects formed within SLM parts. They concluded that such imaging technique is a feasible method to obtain 3D images of SLM defects accurately. 
Furthermore, Ziółkowski et al. [38] explained the advantage of nondestructive XRCT over traditional metallographic cross-sectional analysis. Siddique et al. [34] used a voxel resolution of $4.8 \mu \mathrm{m}$ to scan the SLM specimen. In addition, the authors performed a twodimensional metallographic study, to calculate the porosity and the authors found that porosity calculated by metallographic studies and three-dimensional tomography had no significant difference considering the critical pores. Kim et al. [39] used X-ray microtomography to study the track changes in the morphology of SLM Ti porous structures at a different stage of post laser melting production. They have demonstrated that $\mu \mathrm{CT}$ can be used not only for $3 \mathrm{D}$ quantification but also as a feedback mechanism to make improvements on initial designs. Van Bael et al. [40] used XRCT as a feedback mechanism to improve the geometrical and mechanical controllability of selective laser melted Ti-6Al-4V porous structures. The mismatch between the designed and the measured properties such as pore size, strut thickness, strut volume, and porosity from the first run were integrated into the second run which helped to minimize the mismatch.

Kasperovich et al. [5] presented the correlation between the process parameters and the porosity formation during SLM fabrication of Ti-6Al-4V parts. The authors showed that two types of void defects were present in SLM parts and the optimization of the process parameters could reduce porosity volume fraction. Circular/spherical pores were formed with the excessive energy input whereas insufficient energy density resulted in elongated, narrow crack-like voids. Besides, there are combinations of power, speed, etc. which would result in the same energy densities. Hence, energy density effect, as well as individual parameters effects, should be investigated to identify the process parameters which have more contributions towards porosity formation. In addition, it is 
also important to characterize the pores, as the pore location and pore size are critical to the stress concentration and pores near to the surface are more critical even if they are small in size [34].

The objective of this study is to understand the characteristics of the keyhole pores resulting from different process settings. The effect of power and speed on pore size distribution and pore depth is also a subject of interest. To achieve the objectives, single tracks with different energy densities, are fabricated inside hollow cylindrical samples. The samples are scanned using a micro-CT $(\mu \mathrm{CT})$ scanner, and the reconstructed images are analyzed. Keyhole pore characteristics such as pore size, pore volume, sphericity, etc. are obtained. Total pore number and pore volume of the pores formed within the single track are also measured. In addition to the energy density effect, the effect of power and speed towards keyhole porosity was investigated, by keeping energy density as constant.

\subsection{Experimental Approach}

\subsubsection{SLM specimen design and fabrications}

Figure 5.1(a) shows the design and dimensions of hollow semi-cylinders samples. The diameter of the sample is limited to $7 \mathrm{~mm}$ to ensure proper transmission of X-ray from the sample during CT scanning. Further, two notches are designed to help identify the endpoints of single tracks which would assist during CT scanning as they are used as a reference to align the sample for CT scanning. Figure 5.1(b) demonstrates the alignment of the single tracks on top of the previously fabricated semi-cylinder. The cylinder samples contain four single tracks within, and the two neighbor tracks are 
formed $0.9 \mathrm{~mm}$ apart. This gap is needed to avoid the denuded area during the track formation [28] as well as to minimize the effect of the residual heat from one track to the another.
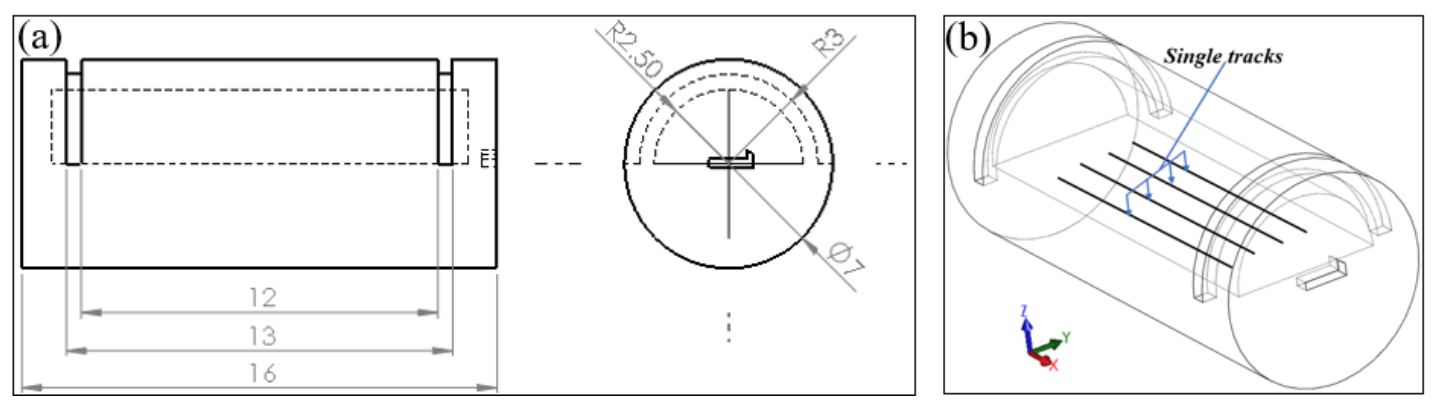

Figure 5.1 (a) CAD model of the specimen (unit in $\mathrm{mm}$ ) and (b) Representation of single track designed on top of previously deposited semi-cylinder.

The Ti-6Al-4V powder with $0-45 \mu \mathrm{m}$ powder size distribution was used to fabricate single track samples using EOS M270 system and the layer thickness during the SLM build was $30 \mu \mathrm{m}$. Argon was supplied to the chamber to maintain the inert atmosphere during the build. The samples were separated from the build plate after the build was complete, and the remaining support structures were then removed by polishing. Figure 5.2 shows the as build samples over the build plate and the samples after the support removal.

(a)

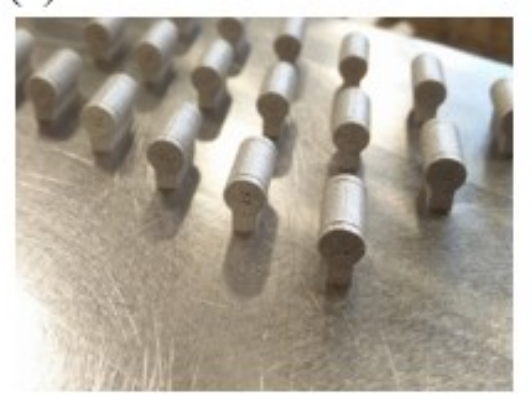

(b)

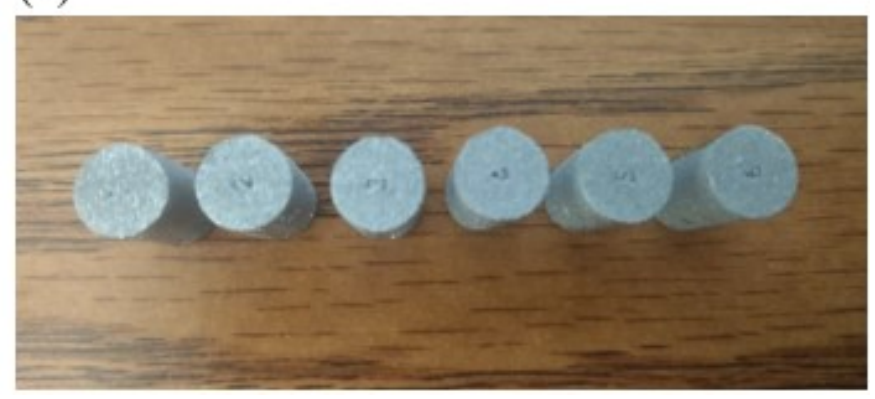

Figure 5.2 (a) Samples after removal from the machine and (b) after support removal. 


\subsubsection{Process parameter design}

Pores formed in the SLM process are generally characterized as keyhole pores and lack of fusion pores [17]. Keyhole pores are formed when the vapor bubbles are trapped within the melt pool, which occurs at higher energy densities, and lack of fusion pores are formed when some regions remain unmelted as a result of lower energy density. Energy density in the SLM process is defined as [29, 30],

$$
E=\frac{P}{v * d * t},
$$

where $P$ is laser power, $v$ is scanning speed, $d$ is hatch spacing and $t$ is the layer thickness.

Line scanning is the foundation of the SLM process, and the combination of line scanning result in the formation of a layer. The study of single-track properties at constant layer thickness may reveal pore formation mechanisms while reducing the variables such as hatch spacing. And the line energy density (LED) can be simplified as [82],

$$
L E D=\frac{P}{v} .
$$

Table 5.1 shows the laser power and the scan speed used in this investigation and their corresponding LED. Three replicates were fabricated to assess the repeatability of the pore formation. 
Table 5.1 Process parameters used to investigate energy density $(\mathrm{J} / \mathrm{mm})$ effect.

\begin{tabular}{|l|l|l|l|l|l|l|}
\hline $\begin{array}{l}\text { Speed }(\mathrm{mm} / \mathrm{s}) \rightarrow \\
\text { Power }(\mathrm{W}) \downarrow\end{array}$ & 200 & 400 & 600 & 800 & 1000 & 1200 \\
\hline 125 & 0.63 & 0.31 & 0.21 & 0.16 & 0.13 & 0.10 \\
\hline 150 & 0.75 & 0.38 & 0.25 & 0.19 & 0.15 & 0.13 \\
\hline 175 & 0.88 & 0.44 & 0.29 & 0.22 & 0.18 & 0.15 \\
\hline 195 & 0.98 & 0.49 & 0.33 & 0.24 & 0.19 & 0.16 \\
\hline
\end{tabular}

LED-based studies deliver the combined effect of the power and the speed on the porosity level. Some studies claim that the use of a specific volumetric energy density (VED) would result in high-density parts $[31,83]$. However, same energy density can be obtained with a different set of power and speed. And the energy density may not be the sufficient criteria to reach that conclusion as energy density is not able to capture the complex physics such as Marangoni flow, hydrodynamic instabilities, and recoil pressure which would dictate the track morphology [84]. Therefore, it is desired to understand the effect of the power and the scan speed on the porosity. For this purpose, a different set of parameters are designed keeping the energy density as constant. Three energy densities $(0.32 \mathrm{~J} / \mathrm{mm}, 0.4 \mathrm{~J} / \mathrm{mm}$ and $0.48 \mathrm{~J} / \mathrm{mm})$ are used to investigate the effect of the power and the scan speed on pore formation as well as overall single-track property. Table 5.2 presents the list of the power and the speed corresponding to the three energy densities. The maximum power of the laser $(195 \mathrm{~W})$ has been used for all cases, and the speed has been modified based on the energy density. 
Table 5.2 Process parameters used to investigate power and speed effect.

\begin{tabular}{|l|l|l|l|l|l|l|l|l|l|l|l|l|l|}
\hline $\begin{array}{l}\mathrm{ED}(\mathrm{J} / \\
\mathrm{mm})\end{array}$ & $\begin{array}{l}\text { Speed( } \\
\mathrm{mm} / \mathrm{s})\end{array}$ & 50 & 100 & 150 & 200 & 250 & 300 & 350 & 406.2 & 450 & 487.5 & 550 & 609.4 \\
\hline $\mathbf{0 . 3 2}$ & \multirow{2}{*}{$\begin{array}{l}\text { Power } \\
\text { (W) }\end{array}$} & 16 & 32 & 48 & 64 & 80 & 96 & 112 & 130 & 144 & 156 & 176 & $\mathbf{1 9 5}$ \\
\cline { 3 - 14 } & 24 & 48 & 72 & 96 & 120 & 144 & 168 & $\mathbf{1 9 5}$ & - & - & - & - \\
\hline $\mathbf{0 . 4 8}$ & & 24 & 100 & 120 & 140 & 162.5 & 180 & $\mathbf{1 9 5}$ & - & - \\
\hline
\end{tabular}

\subsubsection{Pore measurement and analysis}

CT scanning of the fabricated samples was performed using a Bruker SkyScan 1173 micro-CT scanner which has three major components: X-ray source, sample stage and flat panel detector as shown in Figure 5.3(a). The polychromatic X-ray source emits the conical beam and can generate up to $130 \mathrm{kV}$ X-ray. Since the absorption of Ti-6Al$4 \mathrm{~V}$ is very high, the maximum voltage of $130 \mathrm{kV}$ is used during the scan. However, the X-ray being polychromatic, an appropriate filter must be used to absorb low energy Xrays. $0.25 \mathrm{~mm}$ brass filter is used to absorb X-ray with energy below $90 \mathrm{kV}$ which in turn changes the camera sensitivity for X-ray radiation. This is necessary for highly dense materials to reduce beam hardening artifact otherwise the outer surface would appear more dense than interior due to higher absorption of low energy x-rays near the outer surface. Some of the filtered X-ray beams gets absorbed by the sample, and some penetrate through the sample which excites the camera sensor. The typical minimum transmission through the sample lies between 18 and 19\%.

Radiotransparent materials like clay, Styrofoam, and parafilm tapes are used to mount the cylinder sample to the brass stage. The parafilm tape is wrapped around the sample to ensure there is no movement during the scanning. The samples were scanned using $6 \mu \mathrm{m}$ pixel size and 2000 magnification. Rotation step of 0.1 degrees was used, and 
$360^{\circ}$ scanning was performed to obtain 3600 raw images. After obtaining raw images, steps towards reconstruction such as beam hardening correction, ring artifact reduction, misalignment compensation, and smoothing were performed using parameter fine-tuning to minimize the ring artifacts and blurring effects, etc. Reconstruction from scan projection images into cross-section images was carried out using NRecon, which is software from Bruker.

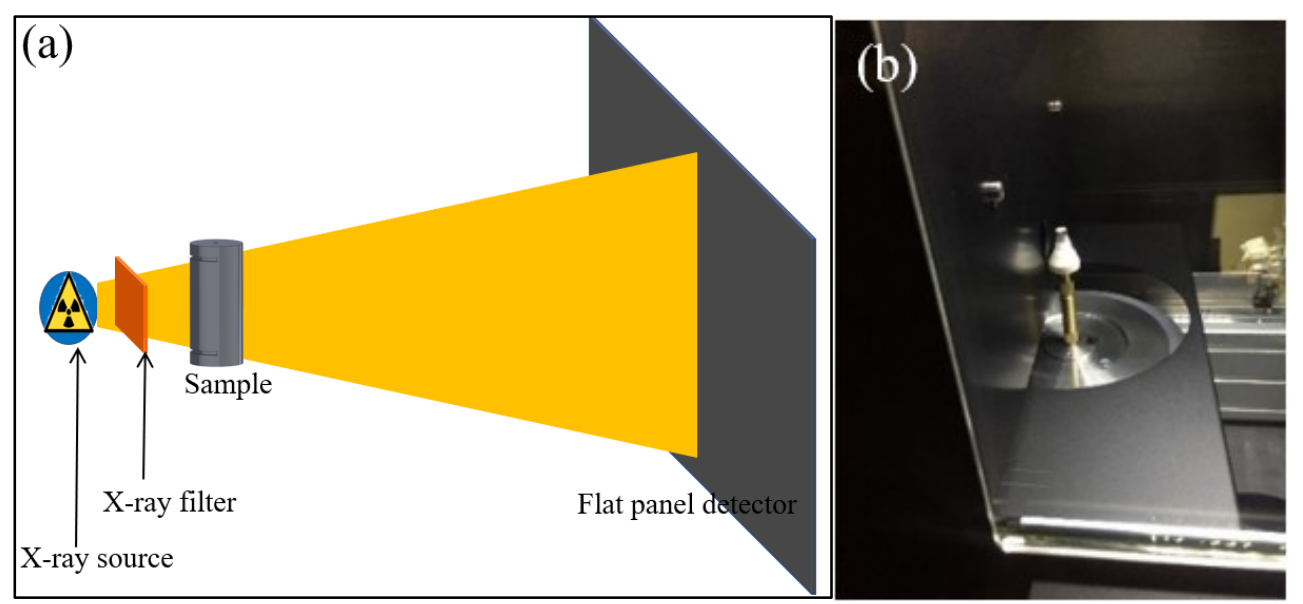

Figure 5.3 (a) Schematic of $\mu \mathrm{CT}$ components and (b) Specimen setup in the CT system

CT-Analyser (CTAn) is also a software from Bruker which is used for measuring quantitative parameters from scanned 3D datasets obtained with SkyScan microCT instruments. For the measurement of keyhole pores, an individual volume of interest (VOI) was generated with a transverse cross-sectional dimension of $400 \mu \mathrm{m} \times 500 \mu \mathrm{m}$, and the length of $13 \mathrm{~mm}$ for every single track as shown in Figure 5.4. Then, the reconstructed images were converted into binary images for pore measurement. Individual pores were analyzed in geometry using CTAn software, and various pore characteristics such as the pore volume, the surface area, the sphericity, etc. were obtained as a result. 


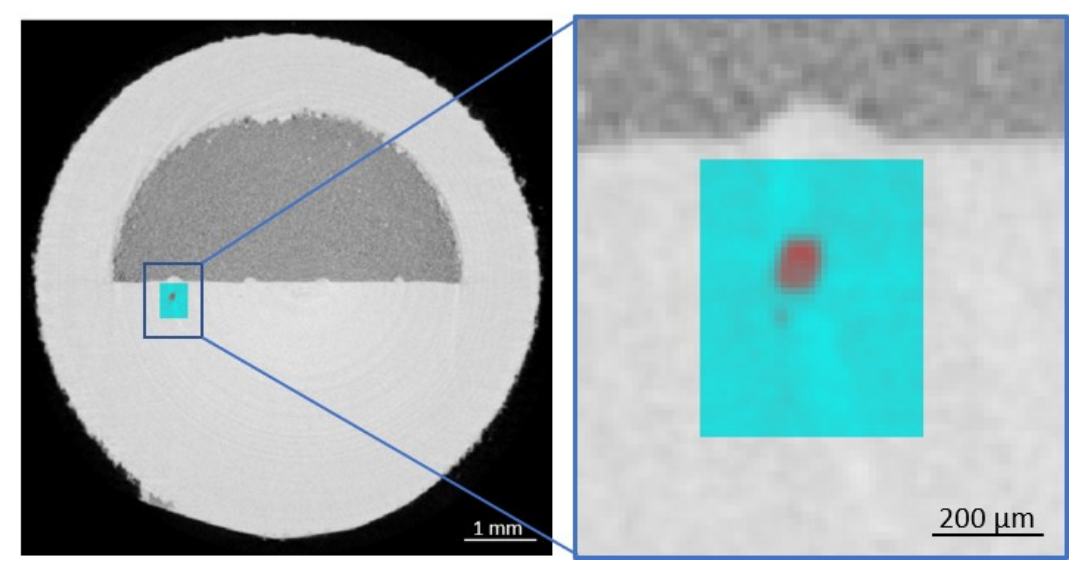

Figure 5.4 Selection of region of interest for porosity measurement.

\subsection{Results and discussion}

\subsubsection{Typical scanning images}

After generating a reconstructed data, Dataviewer software was used to observe the single track formed within the cylinder. Figure 5.5 exhibits the CT images from a typical specimen with different sectional views as well as the isometric partial cutoff view. The color coding shows the density difference between the material, the light grey area represents solid Ti-6Al-4V, the medium grey area represents powder, and the black spots in the sagittal view, which represent the density of air, are pores. Four single tracks shown in the figure were formed with $195 \mathrm{~W}$ power at scan speeds of $200 \mathrm{~mm} / \mathrm{s}, 400$ $\mathrm{mm} / \mathrm{s}, 600 \mathrm{~mm} / \mathrm{s}$ and $800 \mathrm{~mm} / \mathrm{s}$. The sagittal cross-sectional image, obtained from the center of $195 \mathrm{~W}$ and $200 \mathrm{~mm} / \mathrm{s}$ track, clearly shows the pores formed within the single track. Besides the single track, the other solid regions were formed with the power of 170 $\mathrm{W}$, a scan speed of $1200 \mathrm{~mm} / \mathrm{s}$ and hatch spacing of $100 \mu \mathrm{m}$. 


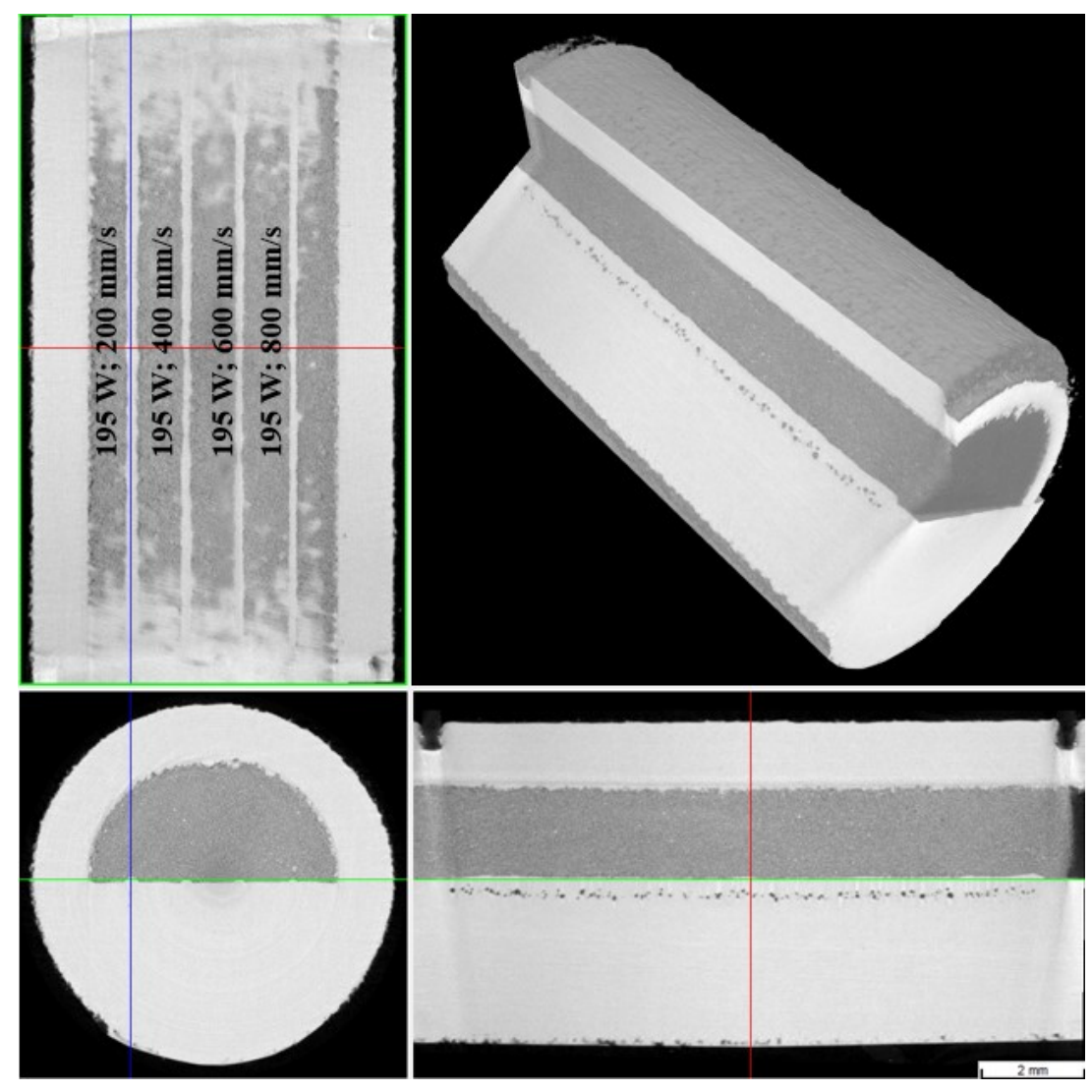

Figure 5.5 (a) Coronal (X-Z), (b) Transaxial (X-Y), (C) Sagittal (Z-Y) cross-sectional views and (d) $3 \mathrm{D}$ partial cut-off view.

\subsubsection{Pore characteristics}

Pore characteristics are obtained from the morphometry analysis of the binary images. In CTAn, the binarization of the images is done by thresholding, which transforms the grayscale images into binary images. The binarized images have been shown in Figure 5.6, where the white pixels represent the pore and the black pixels represent the solid Ti-6Al-4V. Individual pore analysis is then performed to obtain the pore information such as pore volume, pore surface area, sphericity, etc. 3D pores are 
rendered from the series of 2D images. Figure 5.6 presents an illustration of how the 18 successive images are used to form a 3D pore.

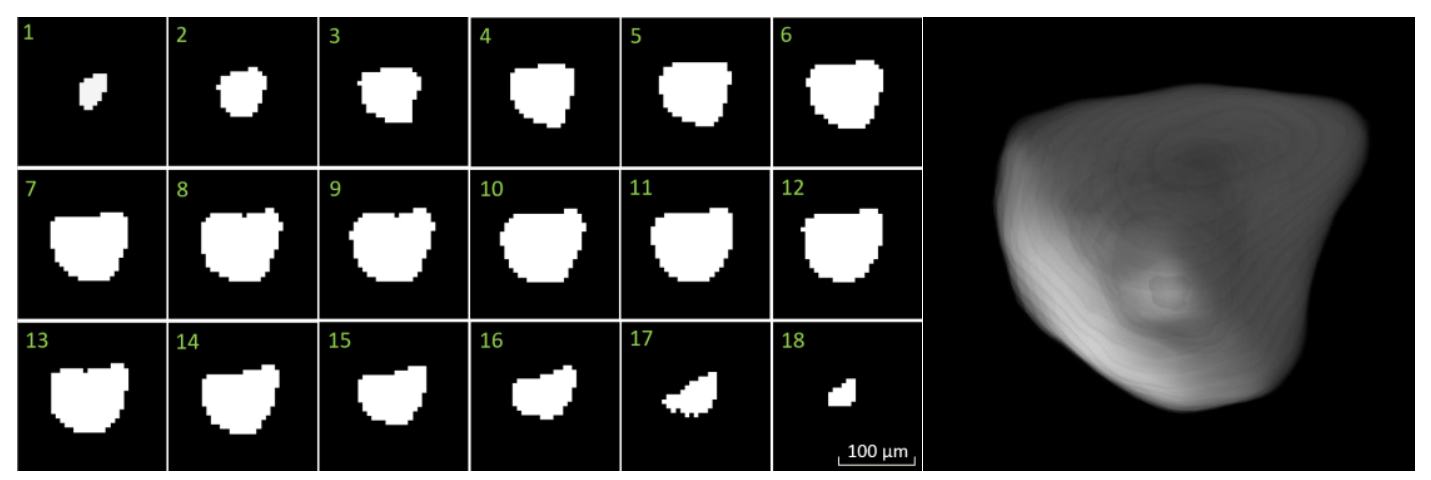

Figure 5.6 A 3D pore on right is rendered from the 2D images from the left.

Sphericity can be used to characterize and differentiate between pores formed due to low energy density and high energy density [5]. Sphericity $(\psi)$, is a measure of how close a body is to the mathematically perfect sphere, which can be calculated by using the relationship

$$
\psi=\frac{\pi^{\frac{1}{3}}\left(6 V_{p}\right)^{\frac{2}{3}}}{A_{p}}
$$

where $V_{p}$ is the volume of the object and $A_{p}$ is the surface area. Pores formed due to lower energy density may be significant during a layer formation or multilayer scanning as the pores are formed due to partial melting. However, during the single-track formation, only keyhole pores are identified, and the discontinuity observed due to lower energy density have not been considered as pores. Figure 5.7(a) shows the reference point used to measure the pore locations. The locations of the pores are measured from the beginning of the scan track $(\mathrm{X})$, the deviation of the pore from the middle of the track $(\mathrm{Y})$ 
and the depth of pore centroid from the substrate free surface over which a single track is formed (Z). Figure 5.7(b) shows twelve pores formed at the end of a single track created with parameters $195 \mathrm{~W}$ and $400 \mathrm{~mm} / \mathrm{s}(0.49 \mathrm{~J} / \mathrm{mm})$ and Table 5.3 shows the detailed characteristics of those pores. The sphericity of all the pores is above 0.8 which mean that they have a nearly spherical shape [5]. These are the pores formed due to very high energy input, and the pores are formed up to $200 \mu \mathrm{m}$ deep which indicated that the pores are the result of deep penetration melting. In addition, the pore centroids formed due to keyhole mode melting are located very close to the center of the laser beam.

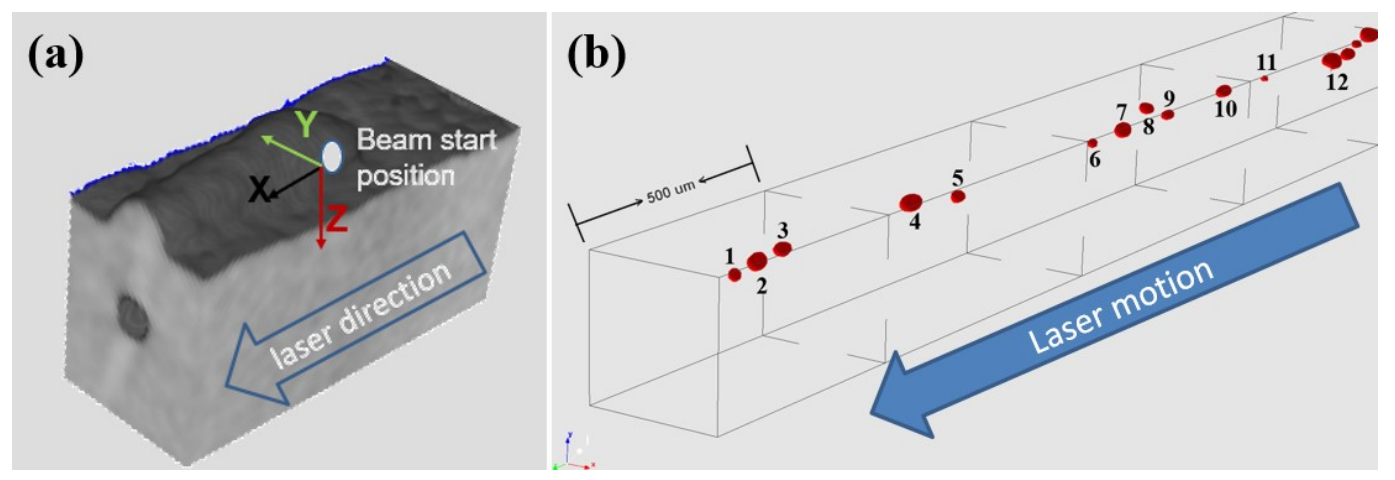

Figure 5.7 (a) Pore location definition and (b) detail view of pores formed at the end of scan track from $195 \mathrm{~W} 400 \mathrm{~mm} / \mathrm{s}$ parameters.

Table 5.3 Location and properties of 9 pores from $195 \mathrm{~W}$ and $400 \mathrm{~mm} / \mathrm{s}$ parameters.

\begin{tabular}{|c|r|r|r|r|r|r|}
\hline $\begin{array}{c}\text { Pore } \\
\text { ID }\end{array}$ & $\begin{array}{c}\text { Volume } \times 10^{5} \\
\left(\mu \mathrm{m}^{3}\right)\end{array}$ & $\begin{array}{c}\text { Equivalent } \\
\text { Diameter }(\mu \mathrm{m})\end{array}$ & Sphericity & $\begin{array}{c}\mathrm{X} \times 10^{4} \\
(\mu \mathrm{m})\end{array}$ & $\begin{array}{c}\mathrm{Y} \\
(\mu \mathrm{m})\end{array}$ & $\begin{array}{c}\mathrm{Z} \times 10^{2} \\
(\mu \mathrm{m})\end{array}$ \\
\hline 1 & 1.38 & 29.7 & 0.89 & 1.17 & 1.34 & 2.02 \\
\hline 2 & 3.96 & 42.3 & 0.88 & 1.15 & -3.33 & 1.88 \\
\hline 3 & 3.15 & 39.2 & 0.89 & 1.14 & -3.29 & 1.84 \\
\hline 4 & 7.11 & 51.4 & 0.89 & 1.09 & 6.04 & 1.58 \\
\hline 5 & 1.60 & 31.3 & 0.82 & 1.06 & -3.72 & 1.81 \\
\hline
\end{tabular}




\begin{tabular}{|c|r|r|r|r|r|r|}
\hline 6 & 1.02 & 26.9 & 0.95 & 0.98 & -16.9 & 1.70 \\
\hline 7 & 4.18 & 43.1 & 0.90 & 0.97 & 1.9 & 1.61 \\
\hline 8 & 2.87 & 38.0 & 0.89 & 0.96 & -2.49 & 1.16 \\
\hline 9 & 1.97 & 33.5 & 0.88 & 0.94 & 4.81 & 1.53 \\
\hline 10 & 3.64 & 41.1 & 0.9 & 0.91 & 14.5 & 1.45 \\
\hline 11 & 0.25 & 16.9 & 0.84 & 0.88 & 17.5 & 1.42 \\
\hline 12 & 9.47 & 56.5 & 0.87 & 0.82 & -10.2 & 1.78 \\
\hline
\end{tabular}

\subsubsection{Process effects}

It has been recognized that laser power and scan speed would affect the singletrack property. The mode of melting during the single-track formation depend upon these parameters and the metallographic studies showed that at high line energy densities keyholes pores are formed [34]. But the number of pores formed at different LEDs is unknown. Therefore, this study aims to find the relationship between the energy density and the keyhole porosity. Besides keyhole pores, pores may also form at the end of the track due to the collapse of the depression upon turning the laser off [35]. Hence, a wide range of LEDs has been selected to investigate the pore formation, and the results are explained in section 5.3.3.1.

In the laser melting process, power is the most significant parameter while determining the penetration depth [36], while laser speed is a significant factor towards the width of the heat affected zone [37]. The behavior of the melt pool would change with changes in the laser power and the scan speed. As the LED does not correspond to the unique laser power and scan speed, it may not be used as an individual parameter to define the optimal processing condition. For the same LED, the level of porosity may 
change depending upon the combination of the laser power and the scan speed. Hence, it becomes important to inquire about the effect of different level of power and speed within the same LED towards porosity. Section 5.3.3.2 explains the effect of the power and speed on the porosity within the same LEDs.

\subsubsection{Line energy density effect}

The energy density, e.g., LED in single tracks determine how much energy per unit length is being supplied to the powder bed system. Thus, the peak temperature of the melt pool as well as the magnitude of depression will change. As a result, the melt pool length, width, and depth will depend on the LED. Figure 5.8 shows the single-track morphology obtained from different LEDs. The results indicated that the track width is proportional to the LED. As the energy density increased, the track width increased. For the same power, the amount of heat input per unit area increases as the scanning speed decreases. The investigated parameters formed a dense track, and no balling behavior was observed. However, for $125 \mathrm{~W}, 1200 \mathrm{~mm} / \mathrm{s}$ discontinuity in the track was observed at certain locations. This may fall within the transition region between conduction mode melting and incomplete melting. 


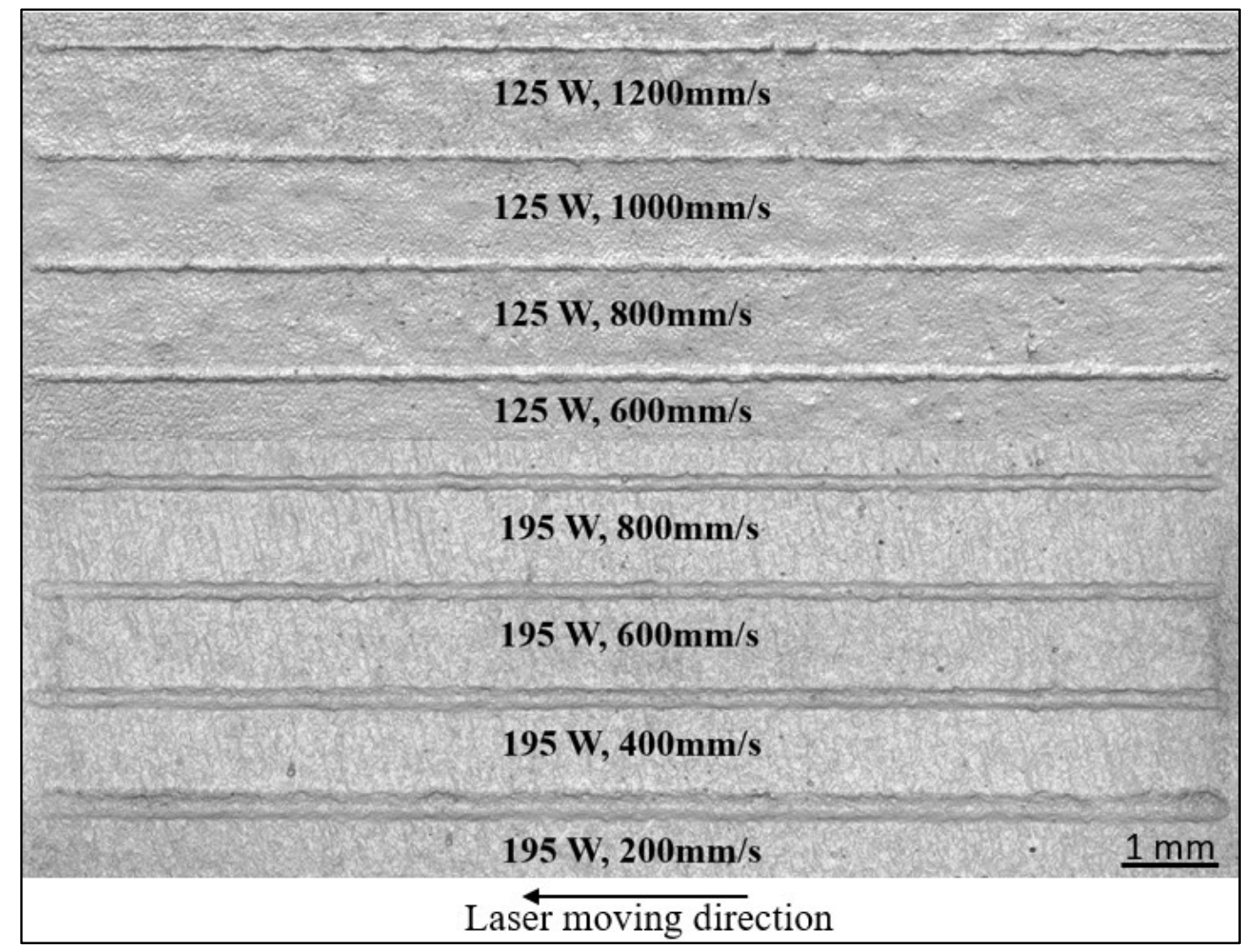

Figure 5.8 Single track morphology from different energy densities.

Figure 5.9 presents the $3 \mathrm{D}$ rendered pores and their location along the single tracks for three cases: $195 \mathrm{~W}$ and $200 \mathrm{~mm} / \mathrm{s}(0.98 \mathrm{~J} / \mathrm{mm}), 195 \mathrm{~W}$ and $400 \mathrm{~mm} / \mathrm{s}(0.49$ $\mathrm{J} / \mathrm{mm})$ and $195 \mathrm{~W}$ and $600 \mathrm{~mm} / \mathrm{s}(0.33 \mathrm{~J} / \mathrm{mm})$. The frequency of the pore decreased with increasing scan speed for the same power level. Pores during deep penetration melting are formed due to keyhole instability [85], and the reduction in pore count may indicate that the severity of keyhole fluctuation is reduced with decreasing energy density. In addition, only 4 pores were observed for $195 \mathrm{~W}$ and $600 \mathrm{~mm} / \mathrm{s}$ compared to 41 pores for $195 \mathrm{~W}$ and $400 \mathrm{~mm} / \mathrm{s}$. This difference may be due to the transition from keyhole mode melting to conduction mode melting. Among the four pores observed for $195 \mathrm{~W}, 400$ $\mathrm{mm} / \mathrm{s}$ case, one pore formed at the end of the track was significantly larger than the other 
three. The pores at the end of the tracks are observed in other low energy density cases as well. When the laser turns off at the end of the single track, the melt pool flows to fill the depression formed during the laser scanning. However, due to rapid solidification, the melt flow may not be able to fill the depression, especially when the depression is deeper as a result of which the pores are formed [64].
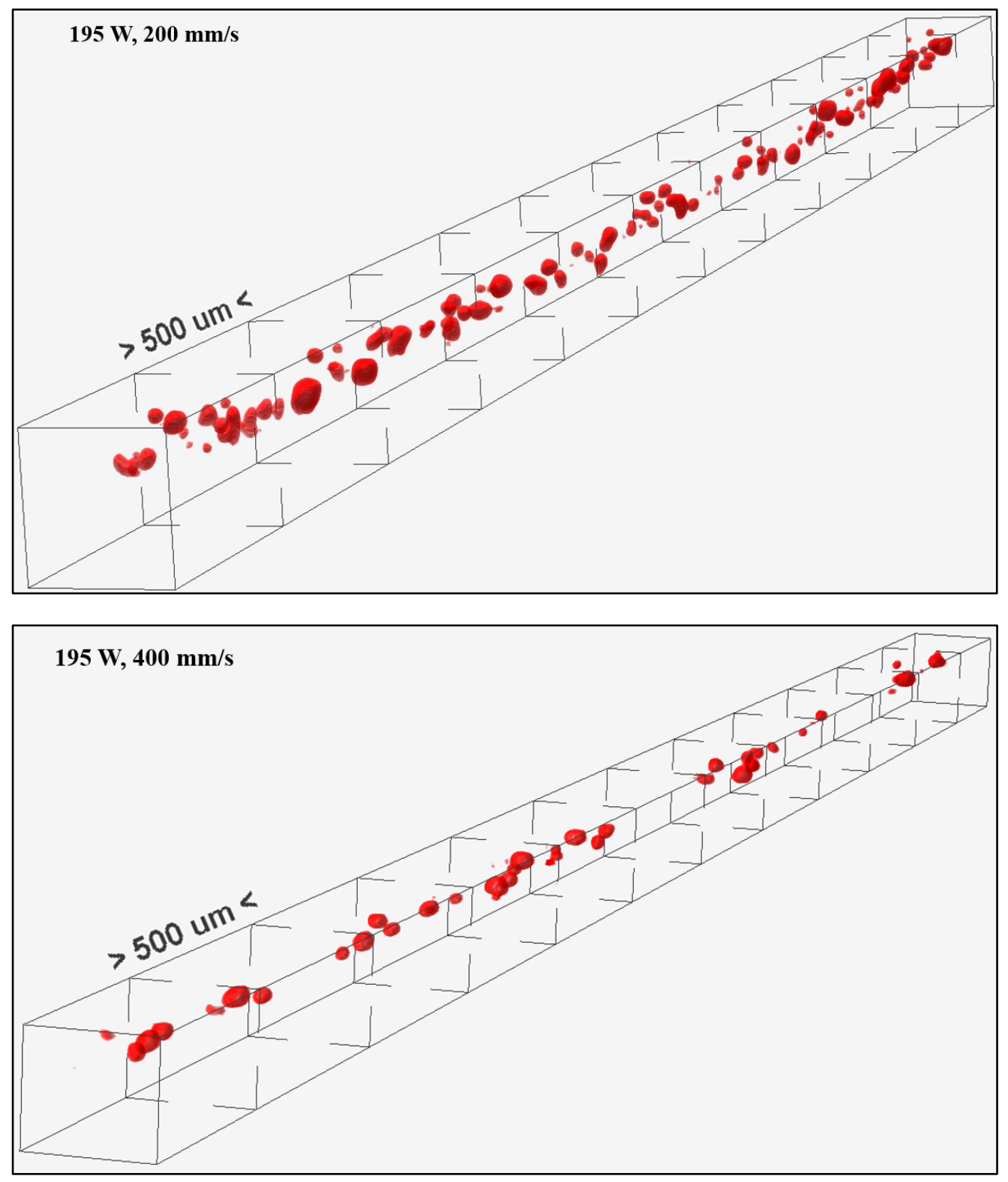


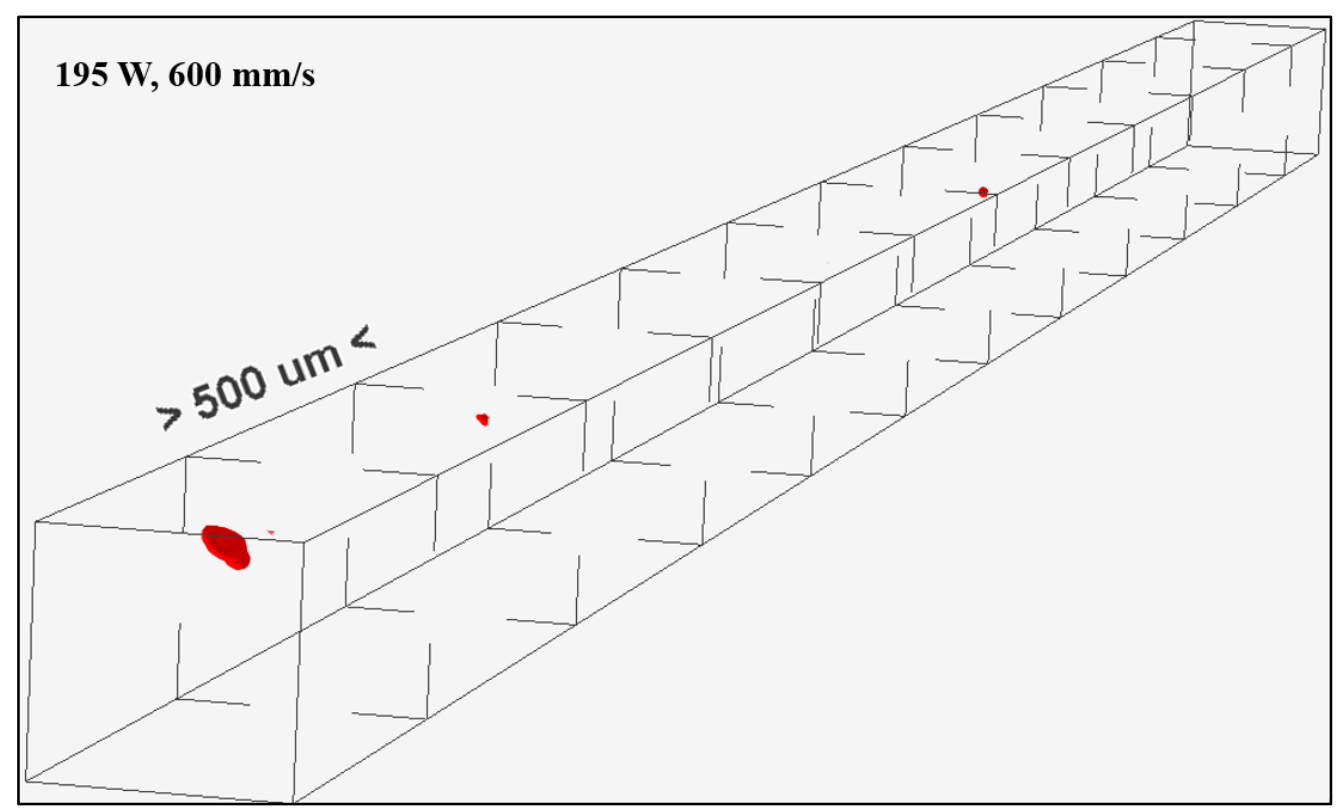

Figure 5.9 3D pore view observed at different process parameters.

Figure 5.10 shows the average diameter and depth of the pores formed with different LEDs. The standard deviation shown in the error bar indicated that there is a huge variation in the size of the pores formed within the same single-track compared to the depth of the pores. In general, as the energy density increased, the average pore diameter and depth increased. High energy density would increase the melt pool temperature due to which material evaporation occurs leading to the formation of a vapor column. As the absorptivity of the melt pool increases with the formation of a vapor column, keyhole depth is also increased [86]. The size of the vapor column depends on the applied intensity, and the collapse of bigger vapor column may lead to larger pores while the collapse of narrower vapor column may lead to smaller pores. For the highest energy density of $0.98 \mathrm{~J} / \mathrm{mm}$, average pore depth is $252.5 \mu \mathrm{m}$. The average diameter of the pores formed as a result was $47 \mu \mathrm{m}$, with the range from $8 \mu \mathrm{m}$ pore to $127 \mu \mathrm{m}$. 
The pores are formed at different depth with different process parameters. It is difficult to explain the melt pool depth resulted from different power level with the study of porosity alone. However, the pore depth would provide an insight on how deep the keyhole penetration is as the metallurgical studies show that keyhole pores are mostly formed at the bottom of the melt pool [36, 87]. And the keyhole penetration increased with increasing energy density.
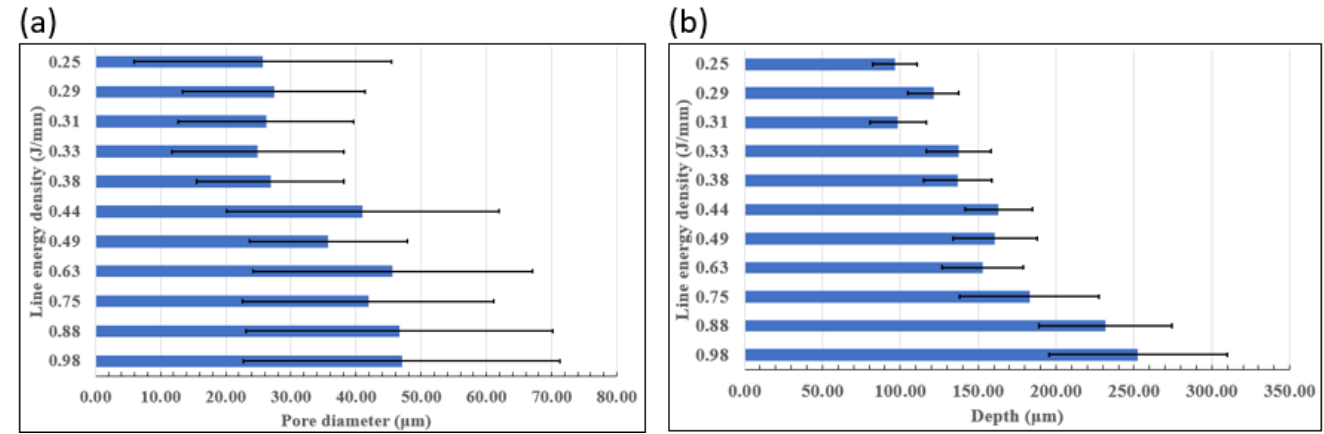

Figure 5.10 (a) Pore diameter and (b) pore depth summary from different LEDs.

Figure 5.11 summarizes the average pore volume and the number of pores formed from the single tracks with 24 different energy densities. The averages were taken from three replicates, and the error bar shows the minimum and the maximum pore number and pore volume obtained from three replicates. The keyhole porosity decreased with decreasing energy density as the magnitude of the keyhole effect decreased. Among the investigated energy densities, the maximum average number of pores and volumes were observed for $0.88 \mathrm{~J} / \mathrm{mm}(195 \mathrm{~W}$ and $400 \mathrm{~mm} / \mathrm{s})$. The pore number is obviously higher for $0.88 \mathrm{~J} / \mathrm{mm}$ compared to $0.98 \mathrm{~J} / \mathrm{mm}(195 \mathrm{~W}$ and $200 \mathrm{~mm} / \mathrm{s})$ however, the range in pore volume showed no clear difference between these two energy densities. Nonetheless, the energy densities with the power of $175 \mathrm{~W}(0.44 \mathrm{~J} / \mathrm{mm}$ with $400 \mathrm{~mm} / \mathrm{s}$ and $0.29 \mathrm{~J} / \mathrm{mm}$ with 
$600 \mathrm{~mm} / \mathrm{s}$ ) resulted in higher number as well as volume of pores compared to the power of $195 \mathrm{~W}$ with respective scan speeds $(0.49 \mathrm{~J} / \mathrm{mm}$ with $400 \mathrm{~mm} / \mathrm{s}$ and $0.33 \mathrm{~J} / \mathrm{mm}$ with $600 \mathrm{~mm} / \mathrm{s}$ ). These results influenced the study of power and speed effect towards keyhole porosity.

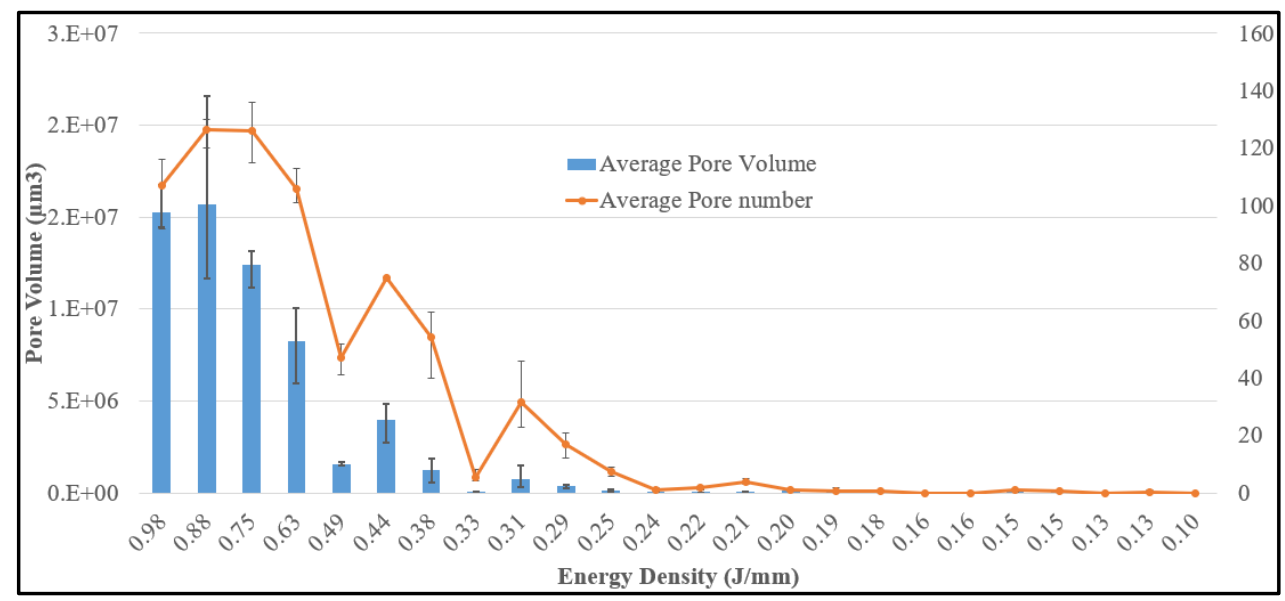

Figure 5.11 Total pore volume corresponding to different energy densities used.

The results are further interpreted based on the power and the speed with Figure 5.12. A plot has been generated to identify the variation in average pore volume within the same scan speed. The trend of porosity is repeated, as for the same power, the porosity level decreased with increasing scanning speed. In addition, for the same speed, increasing the power would increase the pore volume with an exception from $175 \mathrm{~W}$ to 195 W, which may have some relationship with the keyhole depth. It has been experimentally observed that, for the same speed, increasing the laser power would increase the keyhole depth which may induce more keyhole pores [88]. 


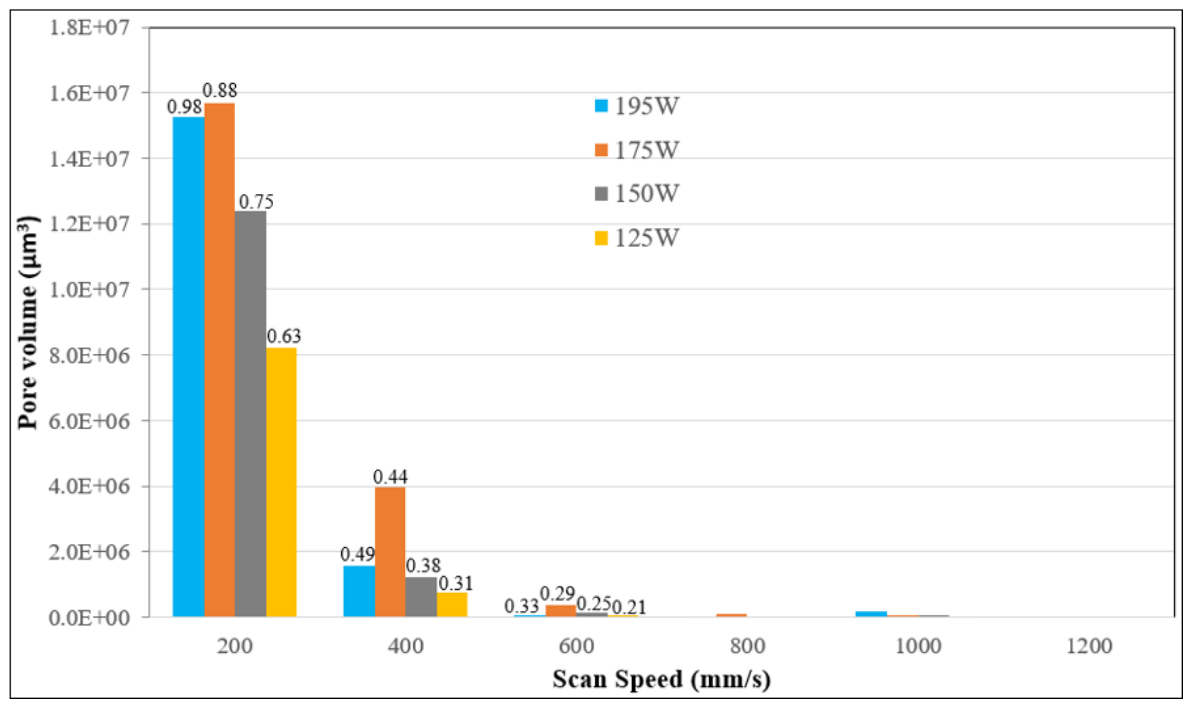

Figure 5.12 Average pore volume at different power and speed.

After analyzing the energy density effect on porosity, it was observed that the pore number and pore volume generally increased with increasing energy density. However, there is a significant drop in pore number and pore volume when LED increased from $0.31 \mathrm{~J} / \mathrm{mm}$ to $0.33 \mathrm{~J} / \mathrm{mm}$ and $0.44 \mathrm{~J} / \mathrm{mm}$ to $0.49 \mathrm{~J} / \mathrm{mm}$. In addition, the average pore number and pore volume also decreased when LED increased from 0.88 $\mathrm{J} / \mathrm{mm}$ to $0.98 \mathrm{~J} / \mathrm{mm}$. The energy densities where the drop in the porosity occurred had the same power of $195 \mathrm{~W}$. These results suggest that energy density may not be an enough criterion to determine the porosity level, as power seems to conribte more towards porosity. Moreover, it has been reported that the volumetric energy density is not an appropriate metric to quantify melt pool depth [84] which may also contribute towards porosity. Hence, individual effects of power and speed of the same energy have been investigated. 


\subsubsection{Effect of power and speed at same energy density}

Based on the LED effect study, three energy densities which resulted in the keyhole pores have been selected to study the effect of the power and the speed within the LEDs. The single-track morphologies obtained from a different combination of laser power and scan speed (LED $=0.48 \mathrm{~J} / \mathrm{mm}$ ) have been presented in Figure 5.13(a). The average widths from all the tracks were measured, and the range of the track width and the averages have been summarized in Figure 5.13(b). The track morphology shows that when the power level is low, a discontinuous track is formed even at a higher energy density. This behavior is like that of the low energy density cases of an incomplete melting regime. When a low power is used, the applied energy is not enough to form a fully developed melt pool, and partial melting leads to the balling formation. The balling formation at the high energy density but low power and scan speed may be explained based on the temperature of the melt pool. The peak temperature within the melt pool for a beam moving with a speed $v$ is [36],

$$
T_{\max }=\frac{\sqrt{2} A I \sigma}{k \sqrt{\pi}} \tan ^{-1} \sqrt{\frac{2 D}{v \sigma}},
$$

where $A$ is absorptivity, $\sigma$ is the half width of Gaussian beam at surface, $I$ is the laser intensity which is related to the laser power $(P)$ as $I=P / 2 \pi \sigma^{2}, D$ is thermal diffusivity of the molten material and $k$ is thermal conductivity. Based on the analytical expression presented in Equation 5.4, the peak temperature to a great extent depends on the laser power rather than scan speed. Hence, lowering the laser power within the same energy density would mean lowering the melt pool temperature. If the power is too low, partial melting may occur like that in $24 \mathrm{~W}$ and $50 \mathrm{~mm} / \mathrm{s}$ case. Such discontinuous tracks are 
observed for lower power cases in other energy densities as well: $16 \mathrm{~W}$ and $50 \mathrm{~mm} / \mathrm{s}$ and $32 \mathrm{~W}$ and $100 \mathrm{~mm} / \mathrm{s}$ for $0.32 \mathrm{~J} / \mathrm{mm}$ and $20 \mathrm{~W}$ and $50 \mathrm{~mm} / \mathrm{s}$ for $0.4 \mathrm{~J} / \mathrm{mm}$ energy densities.

As the power and speed was further increased, it resulted in the formation of continuous and wider tracks. $48 \mathrm{~W}$ laser power with $100 \mathrm{~mm} / \mathrm{s}$ speed only resulted in average track width of $146 \mu \mathrm{m}$, whereas track width beyond this power and speed were over $190 \mu \mathrm{m}$. The melt pool width remained indifferent with further increase in the power and the speed. This may be due to the keyhole mode melting as the flow became dominant along the depth which resulted in almost similar track width.

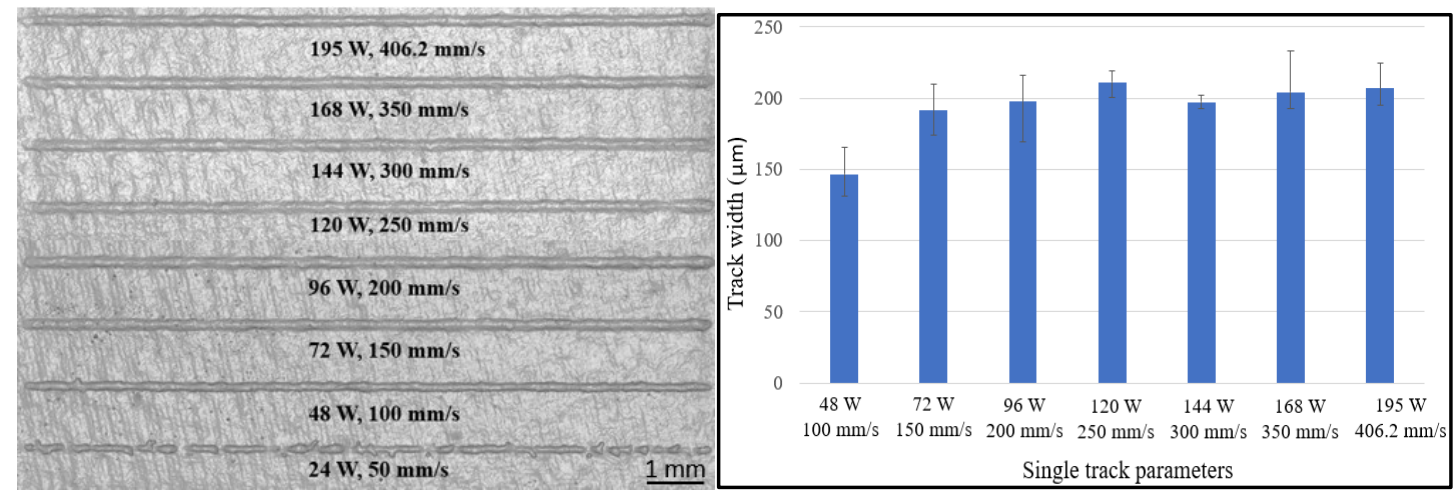

Figure 5.13 Single track morphology at different process parameters for LED=0.48 J/mm.

The longitudinal cross-sections of the single tracks for $0.48 \mathrm{~J} / \mathrm{mm}$ LED have been presented in Figure 5.14. The longitudinal section of the single track with $24 \mathrm{~W}$ and 50 $\mathrm{mm} / \mathrm{s}$ shows wavy profile due to discontinuity in the track. As the power and speed were increased, the waviness was reduced, and continuous, dense tracks were formed. Single tracks with $48 \mathrm{~W}$ and $72 \mathrm{~W}$ did not reveal pores, while as the power increased beyond 72 $\mathrm{W}$, numerous pores were observed. Furthermore, the average depth of the pores increased with an increase in power as shown in Figure 5.15(b). Pore depth has a lower deviation 
compared to pore diameter within the same track. The results show that the characteristics of the keyhole highly depend on the level of power.

The increase in pore depth with increase in power within same energy density may be explained with the non-dimensional depth during laser melting. It has been shown that in the laser welding, the non-dimensional depth of the weld $\left(\delta^{*}\right)$, which is the ratio of depth to the beam diameter, is related to the laser parameters and material properties by the expression [89]

$$
\delta^{*}=f n\left(\frac{A C P}{\rho h_{s} \sqrt{D v \sigma^{3}}}\right)
$$

where $C$ is a constant with no dimensions, $h_{s}$ is enthalpy at melting. From Equation 5.5, it is apparent that the power has more effect on the depth of the melt pool.

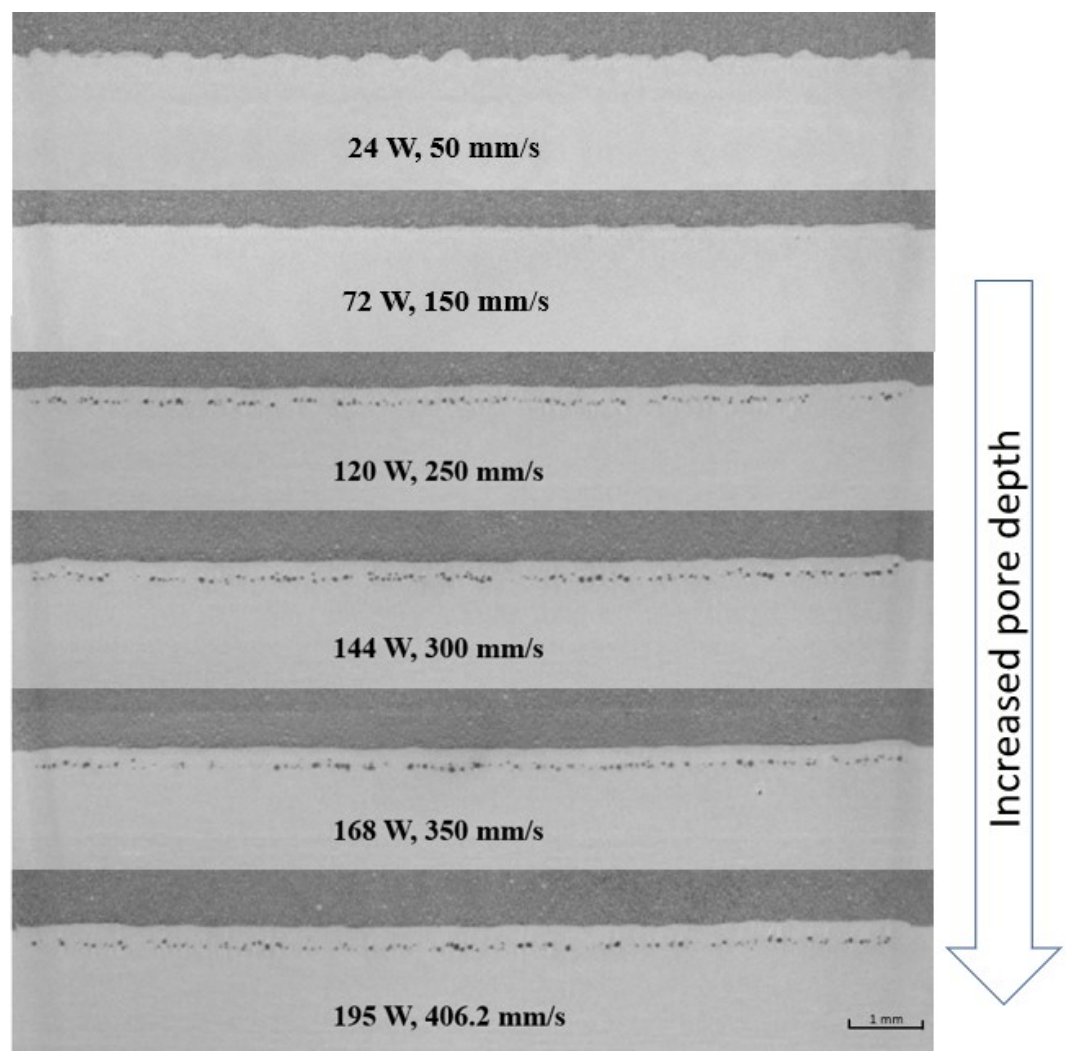

Figure 5.14 Longitudinal sections from the single tracks with $\mathrm{ED}=0.48 \mathrm{~J} / \mathrm{mm}$. 

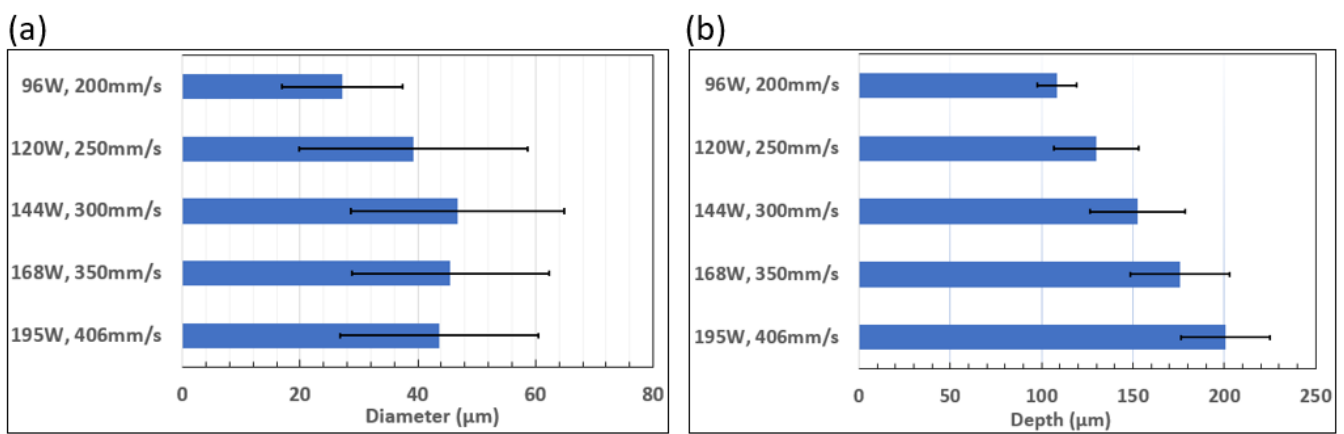

Figure 5.15 Pore diameter and pore depth summary from process parameters of $\mathrm{LED}=0.48 \mathrm{~J} / \mathrm{mm}$.

Figure 5.16 presents the summary of the effect of the power and the scan speed on the pore count and the pore volume. The average pore number and pore volume along with their range obtained from three replicates are presented. It is observed that the level of porosity increased with increasing energy density for the same level of power. In addition, as the power and speed increased for the same energy density, the pore count and the total pore volume increased. As the power would affect the keyhole depth, pore count and volume may increase with increasing power. However, this was not always true for the same energy density as increasing power after a certain level decreased the pore number as well as pore volume. In addition, power appeared to be more significant towards the pore volume as the critical power seems to be similar for all three cases. At low power and low speed, no or very few pores were observed. The pore count increased significantly around the power of $100 \mathrm{~W}$ and continued to increase until $140 \mathrm{~W}$. For 0.32 $\mathrm{J} / \mathrm{mm}$ LED, average maximum volume of pore was observed at $130 \mathrm{~W}$. However, for 0.4 $\mathrm{J} / \mathrm{mm}$ and $0.48 \mathrm{~J} / \mathrm{mm}$, maximum number and volume of pores formed at $140 \mathrm{~W}$ and 144 $\mathrm{W}$ respectively. As the power was further increased, the total pore volume decreased while the depth of the pores increased. 

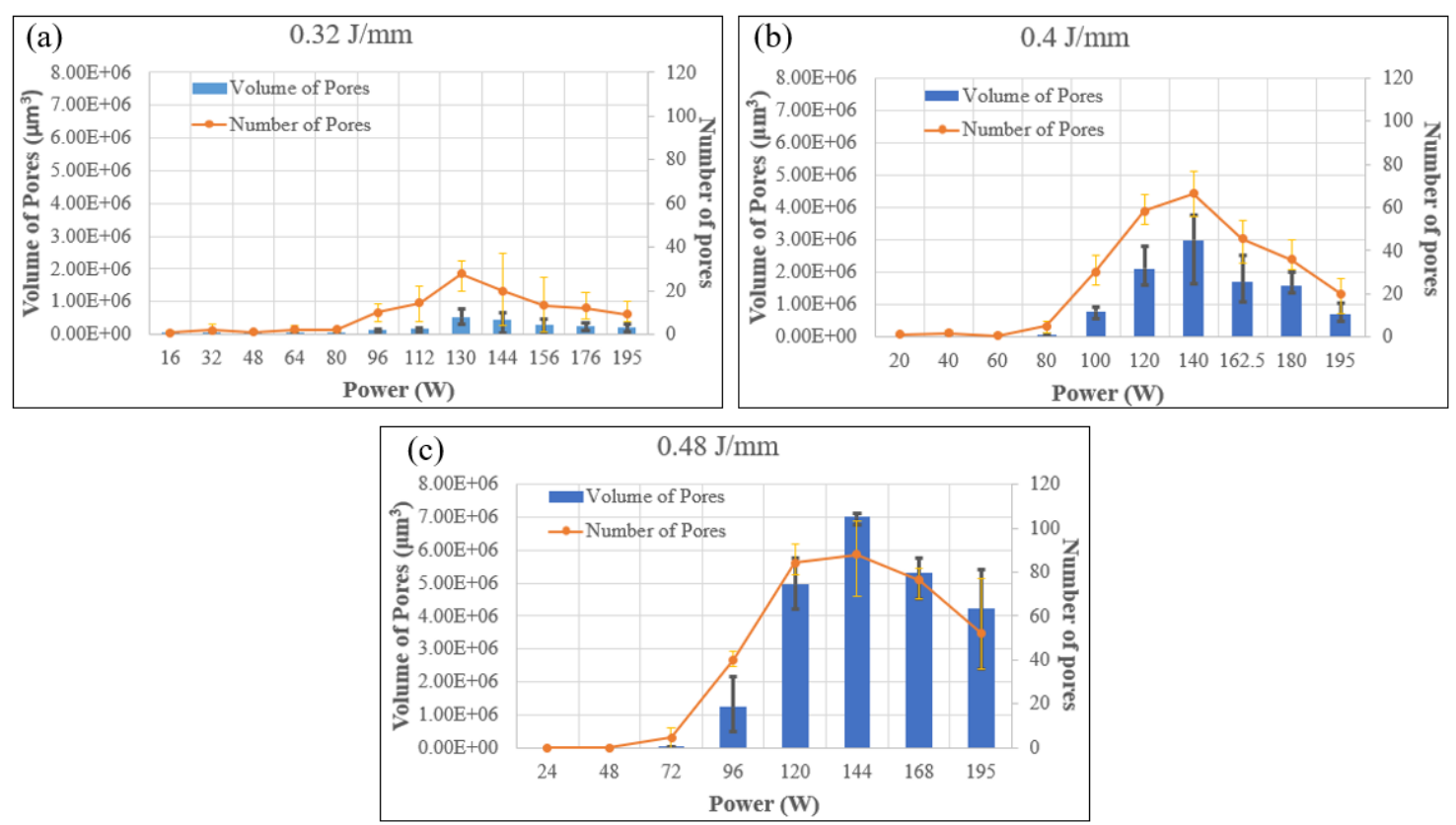

Figure 5.16 Pore count and pore volume obtained at different process parameters from different energy densities (a) $0.32 \mathrm{~J} / \mathrm{mm}$, (b) $0.4 \mathrm{~J} / \mathrm{mm}$ and (c) $0.48 \mathrm{~J} / \mathrm{mm}$.

The observed results in Figure 5.16 may be explained with the help of Figure 5.17, which shows the relationship between the excess pressure due to evaporation $\left(\left(\mathrm{p}+\mathrm{p}_{\mathrm{o}}\right) / \mathrm{p}_{\mathrm{o}}\right)$ and the surface tension pressure, depending upon the normalized keyhole radius $a / \sigma[90]$. The pressure on the keyhole wall $\left(p+p_{o}\right)$ is normalized with ambient pressure $p_{o}$ and the keyhole radius (a) is normalized with laser beam radius $\sigma$.

Equation 5.6 and 5.7 shows the energy balance and pressure balance equations which can be solved numerically to obtain equilibrium keyhole radius [90]. The energy balance equation is,

$$
q_{a b s}=q_{\lambda}+q_{a b l}
$$

where, $q_{a b s}$ is the absorbed energy flux density, $q_{\lambda}$ is the heat conduction losses and $q_{a b l}$ is the energy carried away by evaporation. And the pressure balance equation is, 


$$
p_{a b l}(a)=p_{\Upsilon}(a)
$$

where, $p_{a b l}(a)$ is the evaporation pressure and $p_{\Upsilon}(a)$ is the surface tension pressure. Based on these, there exist two equilibrium states as shown in Figure 5.17: point A and point B. Point $\mathrm{A}$ is called an unstable equilibrium as the surface tension exceeds evaporation pressure to its left which leads to collapsing keyhole, while to the right, evaporation pressure exceeds the surface tension pressure and keyhole will expand to equilibrium state B. Furthermore, five different evaporation pressure curves are shown for different absorbed laser power per thickness $\left(P_{a b s} / d\right)$. It shows that there exists a threshold value of the absorbed laser power per thickness (curve 3) below which a keyhole cannot exist. This would explain the formation of keyhole pores at higher power and speed and incomplete melting at lower power and speed which has been shown in Figure 14. That is, for the same energy density, when the power and speed keep on increasing, the melting mode changes from partial melting to conduction melting to keyhole melting. In addition, the probability of pore formation increases around unstable equilibrium, and as the keyhole diameter further increases with an increase in power, the keyhole melting is stable, and the probability of pore formation decreases. This may be the reason for the trend observed in Figure 5.16. As the power was increased to around $140 \mathrm{~W}$, the unstable equilibrium was reached, and with further increase in power, the keyhole became more stable thus reducing the number and volume of pores. 


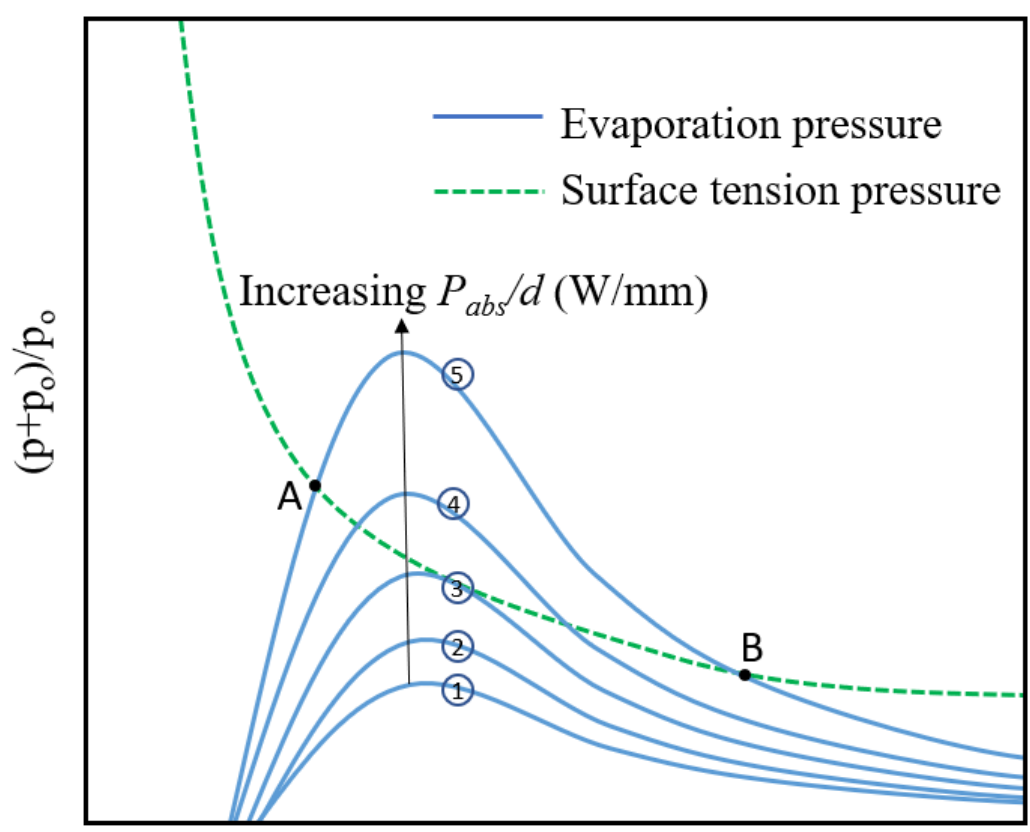

Normalized keyhole radius $(\mathrm{a} / \sigma)$

Figure 5.17 Stability of keyhole formation affected the evaporation pressure and the pressure due to at surface tension at different energy densities [91].

\subsection{Conclusions}

In this study, laser single track scanning was conducted using an EOS M270 and Ti-6Al$4 \mathrm{~V}$ powder with different parameters, total 24 different LEDs, ranging from $0.1 \mathrm{~J} / \mathrm{mm}$ to $0.98 \mathrm{~J} / \mathrm{mm}$, to investigate the relationship between the porosity severity and the LED. Keyhole pores formed in the single tracks were measured using a SkyScan 1173 microCT scanner, followed by data analysis to obatin individual pore geometry and the overall characteristics such as the pore volume, the pore depth, the sphericity etc. Moreover, to investigate the laser power effect under the same energy density on the keyhole pororisty, three energy LED levels, $0.32 \mathrm{~J} / \mathrm{mm}, 0.4 \mathrm{~J} / \mathrm{mm}$ and $0.48 \mathrm{~J} / \mathrm{mm}$, were tested with a wide range of the laser powers. The results and analyses lead to the following conclusions. 
- At the same LED, increasing the laser power shows a distinct transition from incomplete melting to conduction melting, and to keyhole melting. Considering $0.48 \mathrm{~J} / \mathrm{mm}$, at a low power level (24 W and $50 \mathrm{~mm} / \mathrm{s})$, balling formation due to incomplete melting is observed. On the other hand, as the power increase from 96 $\mathrm{W}$ to $195 \mathrm{~W}$, keyhole pores are observed, and the depth of the pores increases with the increasing the laser power.

- Keyhole porosity generally increases with increasing the LED. However, it is observed that the porosity decreases when the LED increases from $0.31 \mathrm{~J} / \mathrm{mm}$ to $0.33 \mathrm{~J} / \mathrm{mm}$, from $0.44 \mathrm{~J} / \mathrm{mm}$ to $0.49 \mathrm{~J} / \mathrm{mm}$, and from $0.88 \mathrm{~J} / \mathrm{mm}$ to $0.98 \mathrm{~J} / \mathrm{mm}$.

- For the pore size, the equivalent pore diameters measured are in a wide range, from $8 \mu \mathrm{m}$ to $127 \mu \mathrm{m}$ and the frequency of larger pores appears higher in higher LEDs, which show increased average pore diameters. In addition, the pores are deeper from the surface with the increase of LED.

- The power/speed effect study shows that the level of the laser power is highly influential towards the keyhole porosity due to its influence on the keyhole stability. For the same LED, e.g. $0.40 \mathrm{~J} / \mathrm{mm}$, the pore number, and the pore volume increase with increasing the power until about $140 \mathrm{~W}$ and then both the pore number and volume decrease, if the power is further increased. This may be due to the stability of the keyhole formation at different laser energy intensities. 


\section{CHAPTER 6 A NUMERICAL STUDY ON THE KEYHOLE FORMATION DURING}

\section{LASER POWDER BED FUSION PROCESS}

\subsection{Introduction}

Laser powder bed fusion (LPBF) process is a metal-based additive manufacturing (AM) technology which utilizes a laser to sequentially melt the powder particles. Due to the line by line and layer by layer scanning process, complex geometries can be fabricated using LPBF. However, the process itself would induce defects due to high thermal gradient and residual heats[50]. The severity of defects formation depends upon the process parameters and hence the proper selection of process parameters is a must.

Several studies have been carried out to find the optimum process window which would give full density parts $[35,91]$. Single track experiment would provide knowledge about the track profile, mode of melting, etc. However, it would require build preparations, part fabrication, metallography, etc. to get all the information which is costly as well as timeconsuming. Hence, predictive simulation models in LPBF are very important as it would reduce the experimental efforts. In addition, simulation tools also help to understand the underlying physical phenomenon which is otherwise difficult to observe from experiments [24].

The melt pool behavior during the single track formation depends on the process parameters selected such as laser power and scan speed [82]. Furthermore, the surface 
quality of the layer would additionally depend on the hatch spacing [23]. Lee and Zhang [25] developed a high-fidelity model by including physics such as the Marangoni effect and recoil pressure, which are important to correctly predict the melt flow during the SLM process. The recoil pressure due to evaporation causes significant depression and its inclusion within the model help predict melt pool depth accurately. In addition, the transverse cross-section profile of the molten pool was found to be relatively symmetric about the center of the laser. In a similar manner, Wu et al. [26] demonstrated that the recoil pressure triggered keyhole formation significantly affects the flow behavior which in turn affect the track morphology. Melt pool predicted with recoil pressure is deeper and narrower compared to the identical model without the recoil pressure. Khairallah et al. [64] incorporated a ray tracing laser heat source to predict the heat transfer and fluid flow during the LPBF process. The single-track simulation explained the complex melt flow as well as the formation of pores and denudation zones. Tan et al. [92] utilized powder scale model to study the evolution of porosity during different modes of melting. It was found that pores formed due to lower energy density were irregular in shape while those formed with high energy density were spherical or elliptical in shapes. Besides, Multiphysics model has been applied to compare the melt pool formation between two different materials: steel and molybdenum [27]. The melt pool size predicted for molybdenum is much smaller than steel due to its higher thermal conductivity.

During the LPBF process, pores may form due to incomplete melting known as lack of fusion pores or due to over-melting known as keyhole pores. When the energy density is very high, material evaporation occurs which leads to the formation of a keyhole. Zhao et al. [93] used ultrafast X-ray imaging for real-time monitoring of LPBF 
process. The static laser source applied to the powder bed system shows the development of keyhole and the formation of pore after the laser turn off. Parab et al. [94] captured the transient behavior of the keyhole using high-speed synchrotron X-ray imaging. The keyhole shapes formed during LPBF of Al-Si10-Mg at Ti-6Al-4V were presented, and the images showed the change in keyhole shape with the change of processing parameters such as laser power and scan speed. Likewise, Cunningham et al. [95] carried out a detail x-ray imaging experiment to observe the keyhole threshold and morphology in laser melting of Ti-6Al-4V alloy. The captured images show the transition from conduction to keyhole mode of melting. The shape and size of vapor depression varies widely across the P-V space. The results also show that the spot size affect the magnitude of vapor depression. These experiments certainly help visualize and understand the powder dynamics, keyhole formation etc., especially the complexity of the process itself to be fully captured by the simulation. In addition, the micro-CT based porosity study has shown that within the same line energy density (LED), the porosity level would depend on the power [96]. As the power increased to $140 \mathrm{~W}$ at LED of $0.4 \mathrm{~J} / \mathrm{mm}$, the pore number increased while there was a drop in the pore number and volume as the power was further increased. It is difficult to explain such results from the experiment only, as the underlying physics is not known, hence high-fidelity simulation would help understand the keyhole behavior during the LPBF process.

In this study, a powder scale model is developed including complex physics such as Marangoni effect, recoil pressure, and evaporation, multiple reflections within keyhole, etc. which are important to predict different modes of melting during LPBF process. The melt pool predicted at different modes: conduction and keyhole melting 
were compared and validated against the experiment, and then the effect of power and speed towards the keyhole behavior has been investigated.

\subsection{Discrete element method}

LPBF is a powder bed AM process. Due to the presence of the porosity between the powders, the laser interaction would be different compared to the flat surface. Ye et al. [97] studied the effect of powder bed thickness on the laser absorptivity and the results indicated that at low power, the difference in absorptivity is significant. Hence, it is important to model the powder particles as there would be multiple reflections of laser rays inside the powder bed. Discrete element method (DEM) is a numerical scheme developed to simulate the behaviour of discrete interacting bodies [98] and therefore is a suitable approach to simulate the powder distribution in LPBF process. An opensource DEM code called LIGGGHTS (LAMMPS Improved for General Granular and Granular Heat Transfer Simulations) is used to simulate the powder distribution process. The powder generation and recoating process of LPBF is simulated by designing a reduced size of the dispenser platform and build platform as shown in Figure 1. The distribution of the powder diameter with range $0-45 \mu \mathrm{m}$ is discretized to get the percentage mass distribution. This discretized particle size range is then utilized to generate the powder particles based on the mass contribution. Due to the use of an explicit time integration scheme and small particle diameters, very small time-step size should be used. The time step is chosen which satisfies the Rayleigh criteria shown in equation 1 [99]. In order to maintain a reasonable time step size, the minimum powder radius used is $10 \mu \mathrm{m}$.

$$
\Delta t=\frac{\pi R_{\min } \sqrt{\rho / E}}{0.163 v+0.877}
$$


where $\Delta t$ is the time step size, $R_{\min }$ is the minimum radius of the powder particles, $\rho$ is the density, $\mathrm{E}$ is the modulus of elasticity and $\mathrm{v}$ is the speed of powder. The material properties of Ti-6Al-4V such as modulus of elasticity, poisons ratio etc. used during the simulation have been summarized in Table 6.1.

Table 6.1 Ti-6Al-4V properties for DEM simulation.

\begin{tabular}{ll}
\hline Parameters & Values \\
\hline Density $\left(\mathrm{Kg} / \mathrm{m}^{3}\right)$ & 4420 \\
Modulus of Elasticity $(\mathrm{GPa})$ & 200 \\
Poisson's ratio & 0.41 \\
Coefficient of restitution & 0.5 \\
Sliding friction coefficient & 0.5 \\
Rolling friction coefficient & 0.1 \\
\hline
\end{tabular}

In the DEM algorithm, all particles are identified separately by their radius, mass, moment of inertia, etc., and all the particles are tracked by solving their trajectories. The general forms of model equations are presented (equation 6.2 and equation 6.3) [100]. The force balance for the particle with index $i$ is

$$
\begin{aligned}
& m_{i} \ddot{x}_{i}=F_{i, n}+F_{i, t}+F_{i, b}, \\
& I_{i} \frac{d \omega_{i}}{d t}=r_{i, c} \times F_{i, t}+T_{i, r}
\end{aligned}
$$

where $F_{i, n}$ is the normal particle-particle contact force, $F_{i, t}$ is the tangential contact force and $F_{i, b}$ is the body force (gravity in this case). The torque due to tangential force is given by $r_{i, c} \times F_{i, t}$, while $T_{i, r}$ is and additional torque on the particle modelled by means of a rolling friction. The particle-particle contact models have been explained in detail in reference [100]. 
The spherical powder particles are generated based on the distribution and dropped into the dispenser platform. During the spreading process, there is an interaction between particles and mesh walls in addition to the particle-particle interaction. Figure 6.1(a) shows the randomly distributed powder particles over the dispenser platform. As the powder is settled, the platform is moved up and recoater is moved towards the build platform. During this process, the interaction between powder particles and build platform determine the powder bed distribution which is depicted in Figure 6.1(b). Besides, the powder distribution over the build platform also depends on several factors like build platform surface, size distribution, an angle of repose, etc. [101]. The distance of $60 \mu \mathrm{m}$ is maintained between the recoater and the build platform considering the maximum diameter of the particle is $45 \mu \mathrm{m}$.
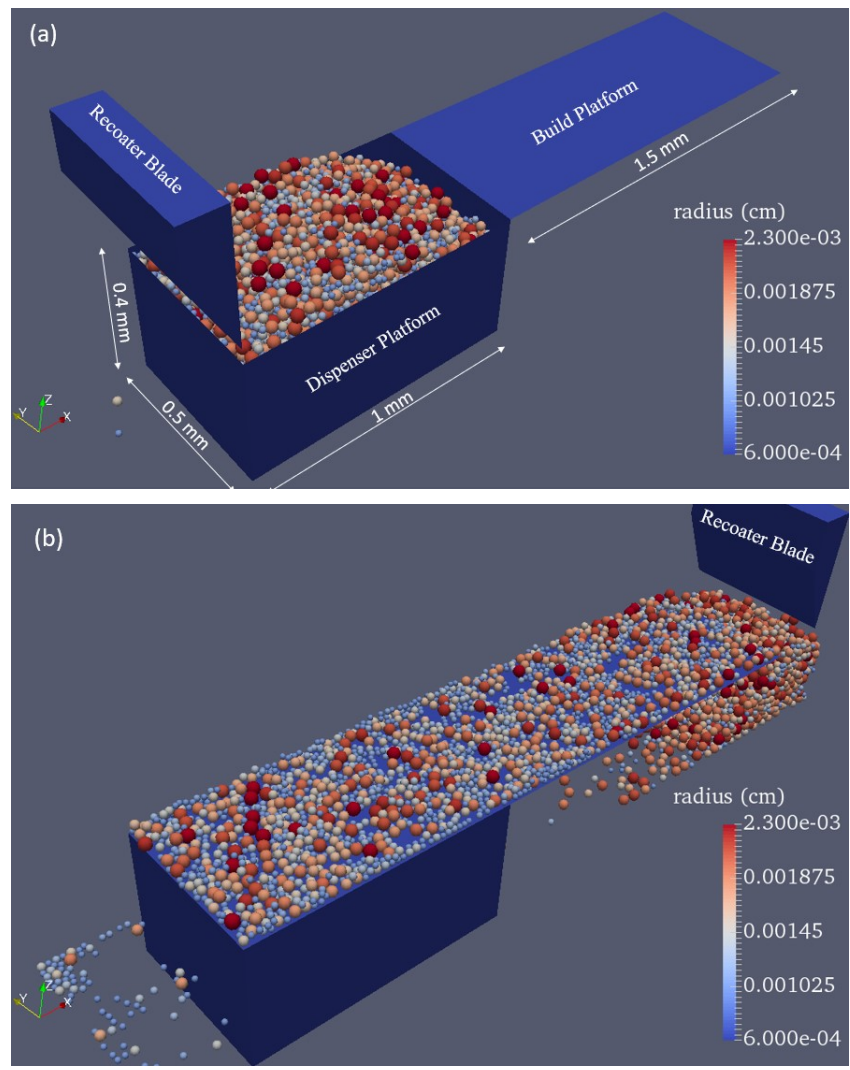

Figure 6.1 (a) Powder added to the dispenser platform and (b) powder particles settled over build plate after recoating process. 


\subsection{Thermo-fluid simulation}

\subsubsection{Simulation domain}

A 3D thermo-fluid model is developed using a FLOW-3D software as shown in Figure 6.2. During the LPBF single track formation, a laser travels along a line with predefined speed. The width and depth of the melt pool depend upon the process parameters applied. Higher energy density would result in a wider and deeper melt pool [87]. This study is focused on keyhole mode melting which is a result of very high energy density. Considering this, a bigger computational domain along depth is required. Hence, a 3D model with domain size $1200 \mu \mathrm{m} \times 400 \mu \mathrm{m} \times 520 \mu \mathrm{m}$ is developed. The powder over the base platform is imported from the LIGGGHTS simulation result. The domain is represented based on the fluid fraction. Powder and solid have fluid fraction of 1 and the rest has 0 fluid fraction. Since the volume of fluid (VOF) is used to predict the surface formation, a hexahedral mesh of $5 \mu \mathrm{m}$ is applied to the computational domain which resulted in close to 2 million cells. In addition, all the walls of the domain are assigned with adiabatic boundary conditions.

During the simulation, the laser was turned on at $200 \mu \mathrm{m}$ in positive $\mathrm{X}$ and $\mathrm{Y}$ directions and total scan length used was $800 \mu \mathrm{m}$. After the laser traveled $800 \mu \mathrm{m}$, it was turned off and the melt pool was let to solidify. 


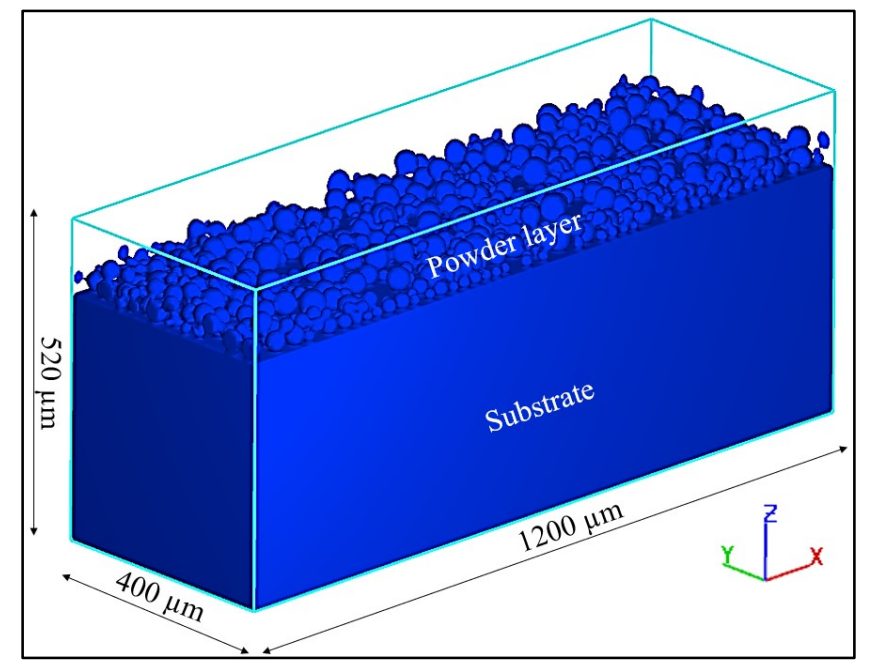

Figure 6.2 3D computational domain used for single track simulation.

\subsubsection{Material Properties}

The physical properties such as thermal conductivity, specific heat, density are the function of temperature. Therefore, the temperature dependent material properties summarized in Figure 6.3 are used to define solid and powder Ti-6Al-4V. In addition, other properties have been listed in Table 6.2.

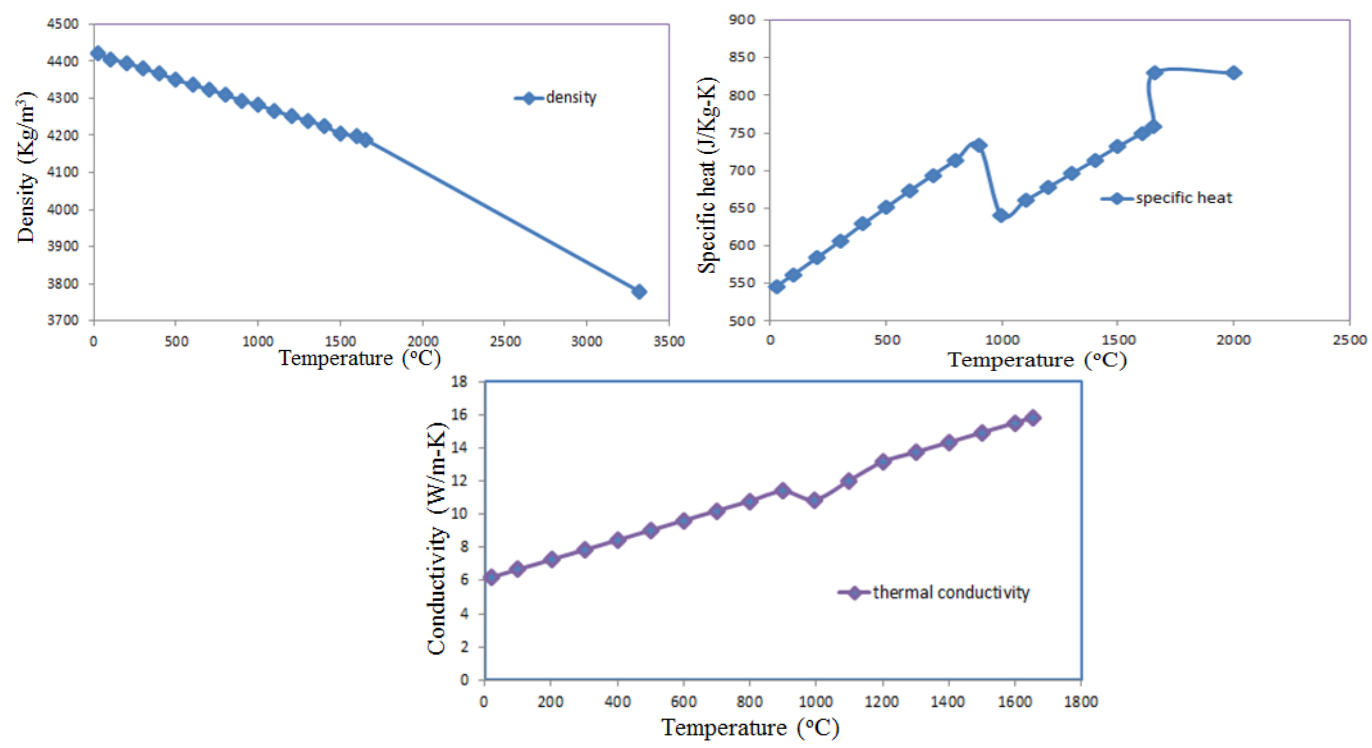

Figure 6.3 Temperature dependent material properties of ti-6al-4v [18]. 
Table 6.2 Properties of Ti-6Al-4V in a thermo-fluid simulation.

\begin{tabular}{ll}
\hline Parameters & Values \\
\hline Solidus temperature, $T_{S}(\mathrm{~K})$ & 1878 \\
Liquidus temperature, $T_{L}(\mathrm{~K})$ & 1928 \\
Boiling temperature, $T_{\mathrm{v}}(\mathrm{K})$ & 3533 \\
Latent heat of fusion, $L_{f}(\mathrm{~kJ} / \mathrm{Kg})$ & 286 \\
Latent heat of evaporation $(\mathrm{kJ} / \mathrm{Kg})$ & 9830 \\
Viscosity $(\mathrm{kg} / \mathrm{m}-\mathrm{s})$ & 0.005 \\
Surface tension $(\mathrm{N} / \mathrm{m})$ & 1.68 \\
Surface tension gradient $(\mathrm{N} / \mathrm{m}-\mathrm{K})$ & -0.00026 \\
\hline
\end{tabular}

\subsubsection{Boundary conditions}

During the simulation, momentum and energy balance equations are solved to predict the fluid flow and temperature, respectively. The heat input is from the laser which has been modeled as a moving Gaussian distribution heat source in the horizontal direction and the equation of the source is presented in equation 6.4.

$$
q(w)=\frac{2 \eta \mathrm{P}}{\pi r^{2}} \exp \left(-\frac{2 w^{2}}{r^{2}}\right)
$$

where $\mathrm{q}$ is the heat flux at a radial distance $\mathrm{w}$ from beam center, $\mathrm{P}$ is laser power, $\eta$ is absorptivity of the material, $r$ is the laser beam radius.

As the heat source is applied, the temperature of the powder and substrate increases, and the powder begin to melt. When the melting point is reached, the flow is primarily governed by the surface tension which is defined as,

$$
\gamma=\gamma_{m}+\frac{d \gamma}{d T} \Delta T
$$

where $\gamma$ is the surface tension, $\gamma_{m}$ is the surface tension at the melting point, $\frac{d \gamma}{d T}$ is the surface tension gradient and $\Delta T$ is the temperature difference. Metals generally have 
negative surface tension gradient and therefore, melt flow occurs from higher temperature region to lower temperature region. Due to the melt flow, the surface deforms with time and the deformed surface is captured by updating the fluid fraction using equation 6.6.

$$
\frac{\partial F}{\partial t}+(V \cdot \nabla) F=0
$$

In addition, when the temperature within melt pool exceeds the boiling temperature, metal evaporation occurs leading to recoil pressure. The pressure boundary condition used in the simulation is,

$$
P_{\text {recoil }}=0.54 P_{0} \exp \left(\frac{\Delta H_{v}\left(T-T_{v}\right)}{R T T_{v}}\right)
$$

where $P_{0}$ is the atmospheric pressure, $\Delta H_{v}$ is the specific enthalpy of metal vapor, $T_{v}$ is the boiling temperature, $\mathrm{T}$ is the surface temperature and $\mathrm{R}$ is the universal gas constant. Equation 6.8 can be simplified as:

$$
P s=A \exp \left\{B\left(1-\frac{T v}{T}\right)\right\}
$$

where $\mathrm{A}=0.54 * P_{0}$, B is a coefficient which is the ratio of latent heat of evaporation to a product of the gas constant and $T_{v}$.

The recoil pressure would increase the melt pool depth due to the formation of vapor depression which may lead to keyhole formation depending upon the magnitude of the recoil pressure. The vapor formation would change the absorptivity within the keyhole as vapor has a different rate of absorption than molten metal. Also, within this keyhole, multiple reflections of the laser occur from the keyhole walls which has been approximated by Fresnel reflection as, 


$$
a=1-\frac{1}{2}\left(\frac{1+(1-\varepsilon \cos \phi)^{2}}{1+(1+\varepsilon \cos \phi)^{2}}+\frac{\varepsilon^{2}-2 \varepsilon \cos \phi+2 \cos ^{2} \phi}{\varepsilon^{2}+2 \varepsilon \cos \phi+2 \cos ^{2} \phi}\right)
$$

where $\phi$ is the angle between the incident ray and the surface normal and $\varepsilon$ depend on material properties and laser type and is related to electrical conductance per unit depth $\left(\sigma_{s t}\right)$ of metal, real part of the dielectric constants $\left(\varepsilon_{1}, \varepsilon_{2}\right)$, the permittivity of vacuum $\left(\varepsilon_{o}\right)$ and $\omega$ represent the angular frequency which is one of the laser properties [86]

$$
\varepsilon^{2}=\frac{2 \varepsilon_{2}}{\varepsilon_{1}+\left[\varepsilon_{1}^{2}+\left(\frac{\sigma_{S t}}{\omega \varepsilon_{0}}\right)^{2}\right]^{1 / 2}}
$$

6.4 Results and discussion

\subsubsection{Typical simulation result}

During the LPBF process, the selection of process parameters would determine the melt behavior. Depending upon the laser power and scan speed, incomplete melting, conduction mode melting, and keyhole mode melting are possible. The following sections will discuss the melt pool behavior during conduction mode and keyhole model of melting. The experimental results are taken as a reference for the selection of the process parameters [87].

\section{Conduction Mode}

In this case, the laser power of $195 \mathrm{~W}$ and scan speed of $1000 \mathrm{~mm} / \mathrm{s}$ is used. Since the melt pool penetration would not be high, the substrate size was reduced, and $200 \mu \mathrm{m}$ thick substrate is used only for this case. Figure 6.4 presents the temperature distribution and the melt flow during laser motion. The red area shown is the melt pool while the laser 
is still in motion and the velocity is maximum at the laser application area which gradually decreases towards the solidification front. Also, the melt pool gradually solidifies as the laser beam moves further away.

A depression is observed at the laser application area which is due to the recoil pressure. But, the recoil pressure at this setup is not enough to develop a keyhole and therefore melt pool is stable. Cunningham et al. [95] performed X-ray imaging during the single track formation of similar process parameters which is presented in their supplementary results. It is interesting to see that the numerical simulation is able to predict the shape of the depression formed at those parameters.

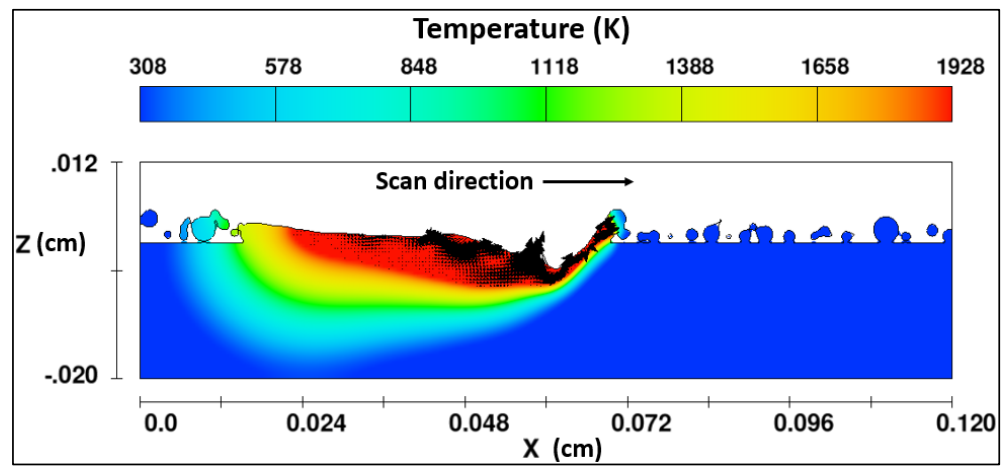

Figure 6.4 Powder and substrate melting during laser application.

The simulation melt pool depth is around $70 \mu \mathrm{m}$ which means that the penetration would be enough to form a strong bond between layers. In addition, no pores are observed during $0.8 \mathrm{~mm}$ scan length simulation due to the stability of the melt pool. The melt region after the single track has completely formed is shown in Figure 6.5. A depressed area is formed at the end of the scan track. Bertoli et al. [84] have explained the transient behavior of the single track at the beginning and end of the scan. Bump is formed at the laser turn-on region and height profile slopes downward at the laser turn off 
region. Figure 6.6 compares the simulation result with the experiment carried out with the same processing parameters[102]. A near semi-circular melt pool shape is predicted with the applied parameters and the melt pool cross-section comparison shows that the simulation can be used to predict the melt pool penetration and boundary during the LPBF process. The melt pool profile is in good agreement with the result from the experiment.

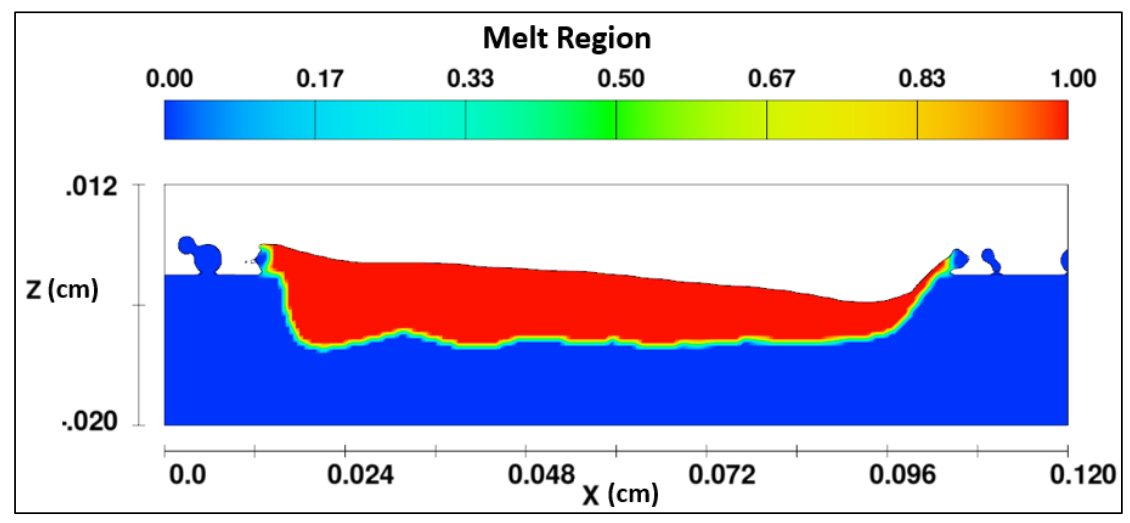

Figure 6.5 Melt region formed after complete melting and solidification.

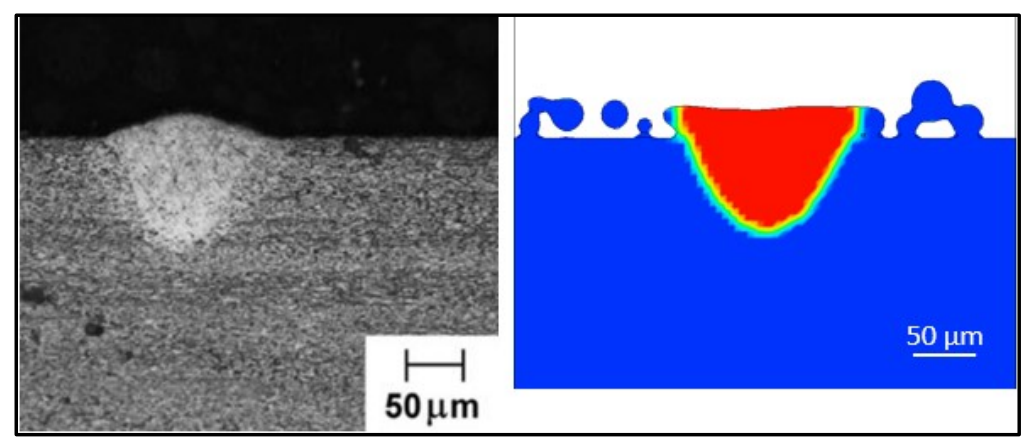

Figure 6.6 Melt pool boundary compared with the experiment [103].

\section{Keyhole Mode}

During the keyhole mode melting, the material evaporation leads to a repulsive force within the melt pool and an opening is formed which is filled with the vapor. The 
evaporation pressure developed would depend on the evaporation flux. This pressure acts to open the keyhole. As the size of the vapor column increases, more vapor is trapped inside it, and the absorption of the keyhole wall further increases. Hence, the depression, as well as the vapor column, continues to grow.

On the other hand, surface tension pressure along with convection induced pressure and hydrostatic pressure act to close the keyhole. However, the magnitude of convection induced pressure and hydrostatic pressure could be neglected due to its weak contribution [90]. As the vapor column becomes wider, the tendency of it to collapse due to surface tension becomes less.

Figure 6.7 shows the relationship between the excess evaporation pressure $\left(\left(p+p_{o}\right) / p_{o}\right)$ and the surface tension pressure based on the normalized keyhole radius $a / \sigma$ [90]. The pressure on the keyhole wall $\left(p+p_{o}\right)$ is normalized with ambient pressure $p_{o}$ and the keyhole radius (a) is normalized with laser beam radius $\sigma$. The two equilibrium states (A and B) shown in Figure 8 are obtained from energy and pressure balance equations given in equation 6.11 and equation 6.12 respectively [90].

$$
\begin{aligned}
& q_{a b s}=q_{\lambda}+q_{a b l}, \\
& p_{a b l}(a)=p_{\Upsilon}(a),
\end{aligned}
$$

where $q_{a b s}$ is the absorbed energy flux density, $q_{\lambda}$ is the heat conduction losses, $q_{a b l}$ is the energy carried away by evaporation, $p_{a b l}(a)$ is the evaporation pressure and $p_{\Upsilon}(a)$ is the surface tension pressure. When the vapor column is below the point $A\left(r_{a}\right)$, the surface tension force can close the vapor column, but if the vapor column continues to grow beyond $\mathrm{A}$, the surface tension force is unable to close the vapor column and the stable keyhole is formed. Due to this, point A is regarded as an unstable equilibrium [90]. As 
the vapor column continues to grow, at a certain point, another equilibrium can be observed which is represented by point $\mathrm{B}$. This is a stable equilibrium where the surface tension would act dominantly to close the vapor column if it further grows.

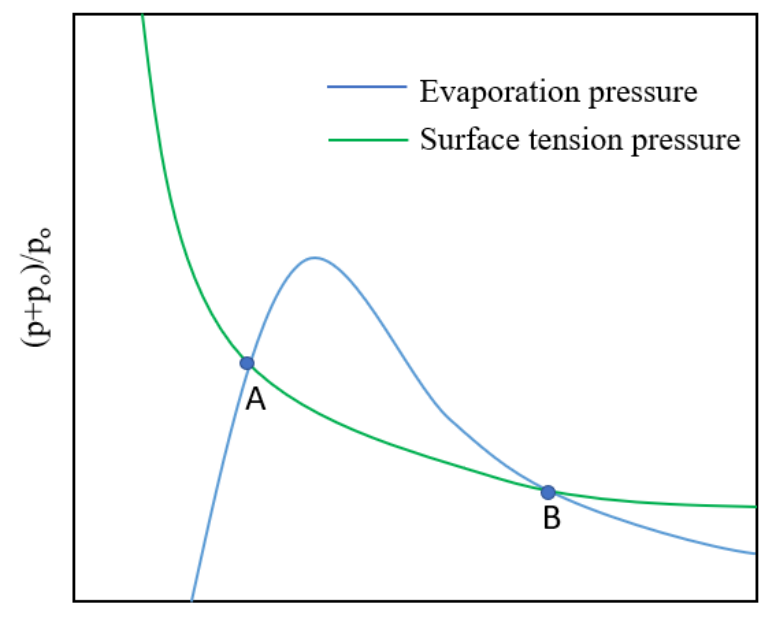

Normalized keyhole radius $(\mathrm{a} / \sigma)$

Figure 6.7 Equilibrium points during the formation of vapor column [91].

A high line energy density has been used to observe the keyhole mode of melting. $195 \mathrm{~W}$ laser power and $400 \mathrm{~mm} / \mathrm{s}$ scan speed is used for this purpose as it would result in pores due to keyhole melting [103]. As the energy density is very high, the temperature within the keyhole exceeds the evaporation temperature, and material evaporation leads to the recoil pressure. Hence, vapor depression is formed which further enhances the laser absorption due to multiple reflections through the keyhole walls. This causes more vapor to form and keyhole depth increases. Figure 6.8 presents the formation of the depression which shows the changing multiple reflections to the change in the surface morphology. At $20 \mu$ s, the penetration is not deep, however as the material is continuously irradiated with the laser, there is an increase in the depression. During this period, there is a high interaction between evaporation pressure and surface tension. A surface tension force tries to close the vapor gap developed which can be observed at $140 \mu \mathrm{s}$. But, due to the 
high intensity of the power, the keyhole remains open during most of the melting process. However, the keyhole closes as the vapor column reaches close to the equilibrium. Due to this, pores are formed, and some of them are trapped in the solidification front.

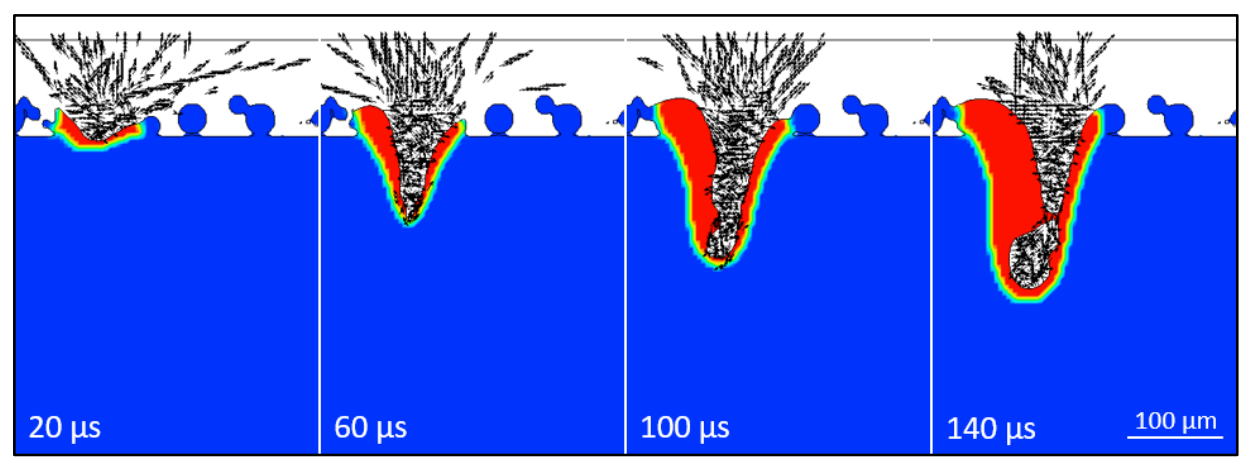

Figure 6.8 Multiple reflection vectors from the keyhole wall.

Figure 6.9 illustrates the formation of pore during the keyhole mode melting. Figure 6.9(a) shows the transient keyhole behavior. At $300 \mu \mathrm{s}$, the keyhole formed remains open, and with the further development of the keyhole, the surface tension force becomes dominant over the evaporation pressure which tries to close the vapor column. When the force is enough to break the column, a bubble is formed as seen at $360 \mu \mathrm{s}$, with strong velocity field around the bubble. These bubbles are either trapped in the solidification front, or they collapse. At $390 \mu \mathrm{s}$, the bubble has collapsed to the forming keyhole. The numerical model can capture the essence of keyhole as well as bubble formation in regard to the high-speed $\mathrm{x}$-ray images discussed in literatures [95, 104]. Figure 6.9(b) shows the temperature distribution and shape of the vapor depression. The keyhole wall temperature exceeds the evaporation temperature. This suggests that there is continuous evaporation of metal. Due to the formation of vapor column, substantial displacement of liquid of liquid from the center of the spot occurs and the liquid moves to the melt pool away from the laser center. Due to this, a surface wave is formed at the rear 
of the melt pool since the flow is dominant towards the keyhole rear wall. The formation of surface wave would play a detrimental role in the morphology of the single track. A bubble trapped at the solidification front is also identified. The bubbles which are trapped are the pores which remain within the single track after it is fully formed.
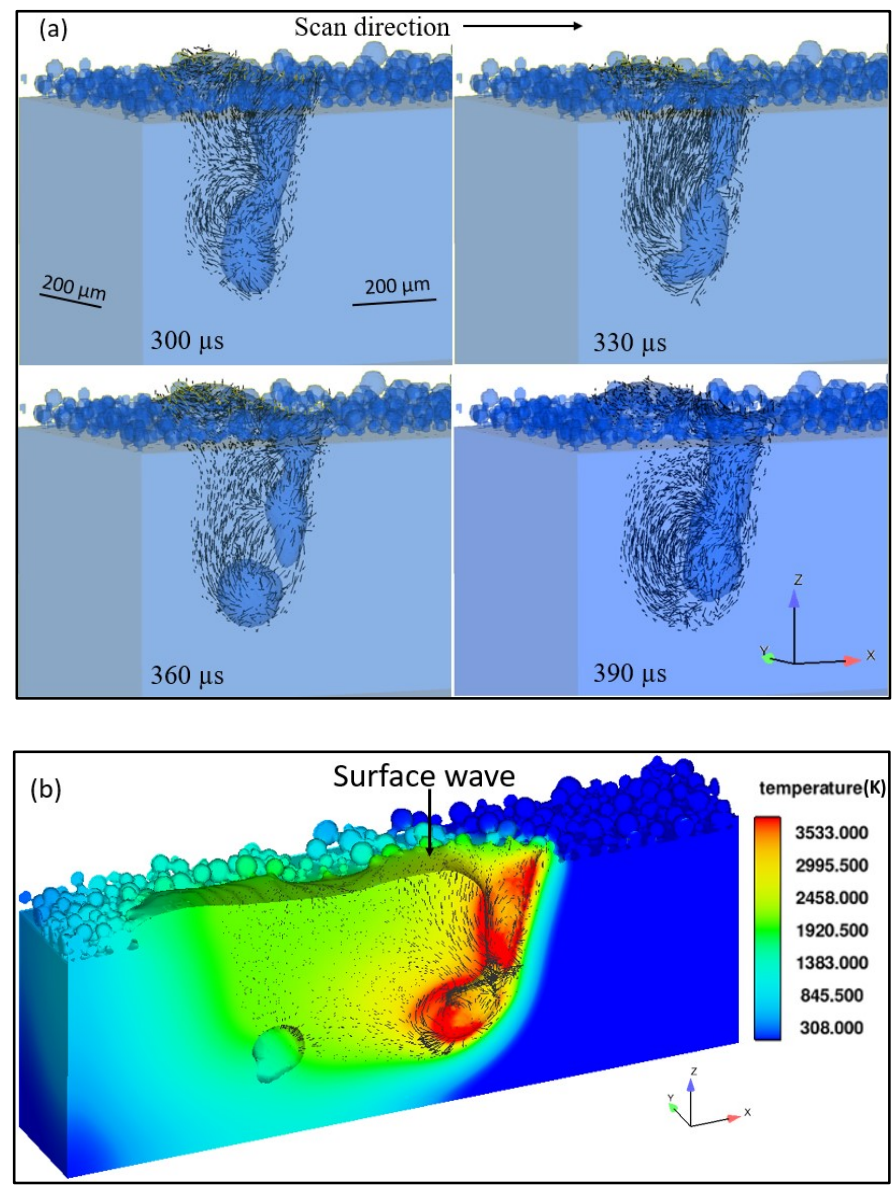

Figure 6.9 (a) Velocity field, keyhole profile and breakage of keyhole to form bubble, and (b) 2D temperature and velocity field along longitudinal section.

The formation of the transverse profile of the melt pool is explained with Figure 6.10. At $260 \mu \mathrm{s}$, when the laser is just above, a depression is formed, which continues to grow $(300 \mu \mathrm{s})$. As the laser beam moves away, the melt flow is inward which tend to close the depression as seen in $330 \mu \mathrm{s}$. After some time, a fully solidified transverse 
keyhole profile is obtained. The simulated keyhole profile has been compared with the experimental result from literature in Figure 6.11 [87]. The developed numerical model can predict the keyhole shape formed due to high energy density. The transverse profile is in good agreement with the experimental transverse profile.

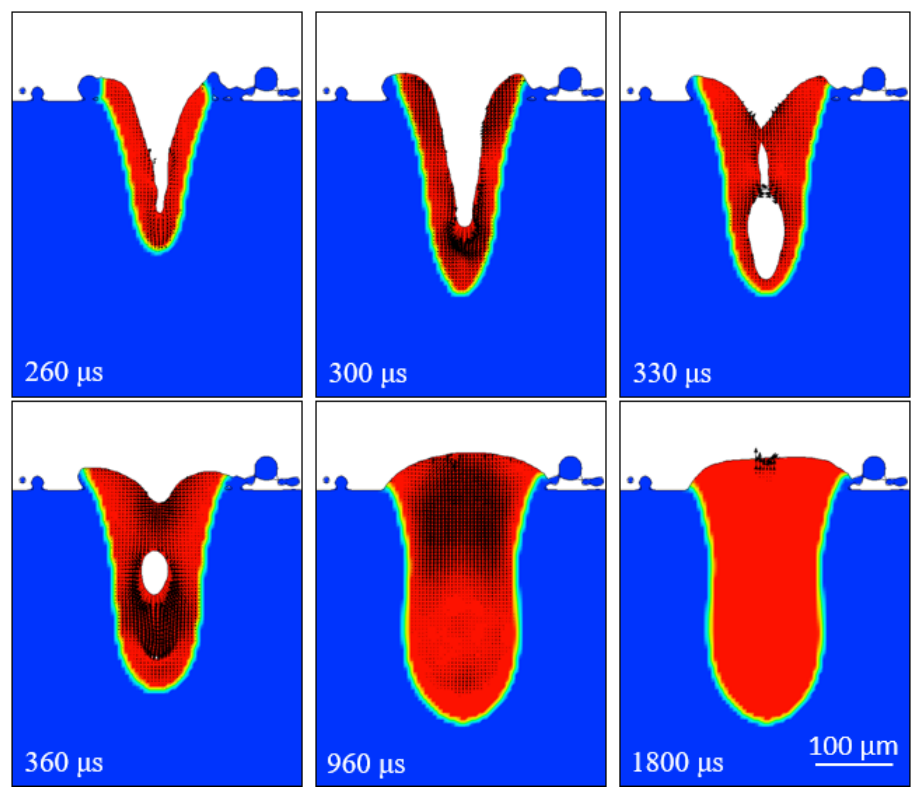

Figure 6.10 Fluid flow in transverse direction during keyhole melting.

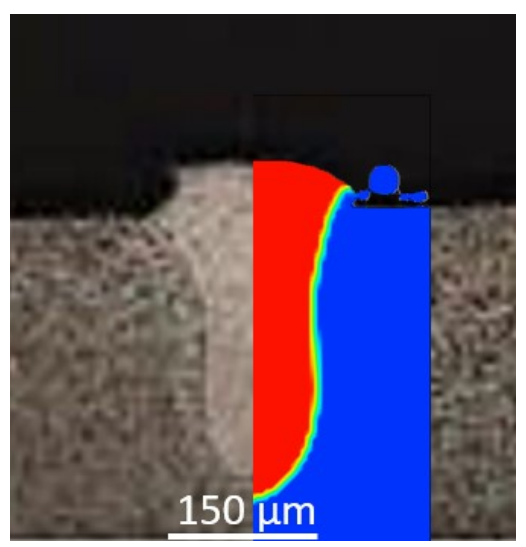

Figure 6.11 Melt pool boundary compared with the experiment for $195 \mathrm{w}$ laser power and $400 \mathrm{~mm} / \mathrm{s}$ scan speed [88].

The final single track after solidification is shown in Figure 6.12. Several pores are observed within the tracks. These are the pores which were trapped within the 
solidified melt region. This suggests that the keyhole pores are formed when the process parameters resulting in high energy density is used.

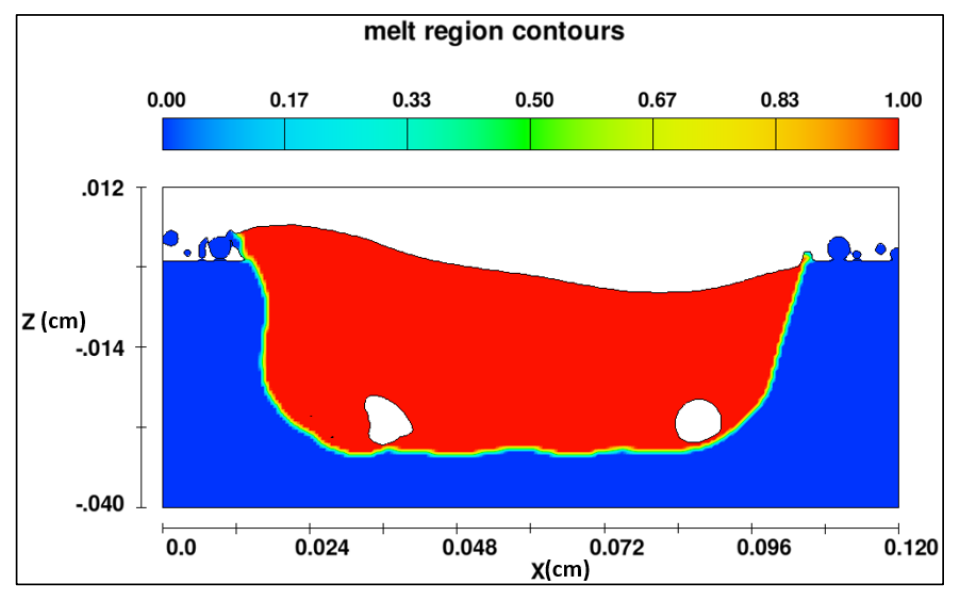

Figure 6.12 Melt region formed after complete melting and solidification.

A single track experiment with the same laser parameters had been carried out using EOS M270 LPBF system [105]. Ti-6Al-4V powder $(0-45 \mu \mathrm{m})$ was used to form a $12 \mathrm{~mm}$ long single track and micro-CT was carried out to observe the pores formed within the single track. Figure 6.13 shows the cross-section as well as $3 \mathrm{D}$ rendered pores formed at the beginning $3 \mathrm{~mm}$ of a single track. Within this region presented, 10 keyhole pores are observed.

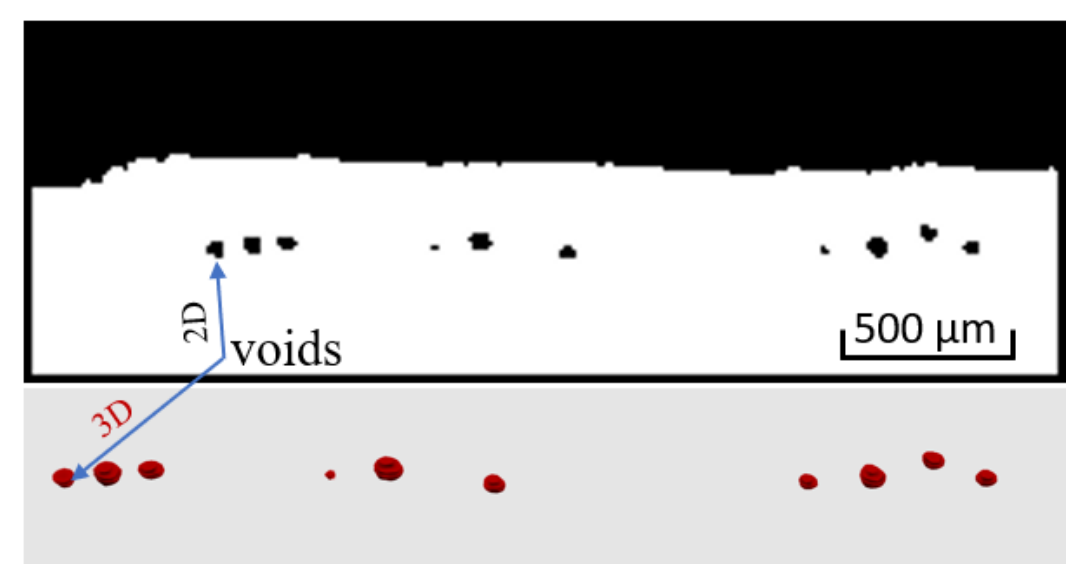

Figure 6.13 2D images of the pores formed at the beginning of the single track and their $3 \mathrm{D}$ rendered morphology. 


\subsubsection{Effect of power and speed on keyhole formation}

Line energy density (LED) is the function of laser power (P) and scan speed (v).

$$
L E D=\frac{P}{v}
$$

Within the same energy density, the behavior of the flow and single track would depend upon the power and speed. The range of optimum scan speed varies with the level of power [82]. An experimental study had been carried out to investigate the effect of power and speed on the level of porosity during the formation of $12 \mathrm{~mm}$ long single tracks [96]. Figure 6.14 presents the pore number and pore volume resulting from the different combination of power and speed with LED of $0.4 \mathrm{~J} / \mathrm{mm}$. The results indicated that the pore number and volume increased to a certain level of power and then dropped with further increase in power at same energy density. This interesting behavior is difficult to explain with experimental study alone and therefore the numerical model has been utilized to understand the physics that leads to such behavior.

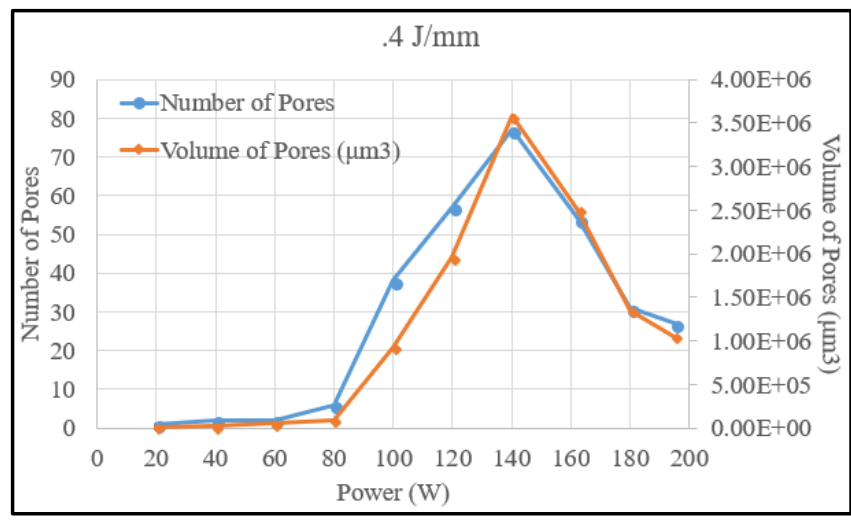

Figure 6.14 Pore number and volume from a different level of power with LED $=0.4$ $\mathrm{J} / \mathrm{mm}[97]$.

Again, LED of $0.4 \mathrm{~J} / \mathrm{mm}$ was used for numerical study which would result in the formation of a keyhole and the corresponding power and scan speed has been listed in 
Table 6.3. A bigger window of power is selected to gain more insight towards the keyhole mode melting as the power used in the experiment was limited to $195 \mathrm{~W}$.

Table 6.3 Power and Speed with LED $=0.4 \mathrm{~J} / \mathrm{mm}$.

\begin{tabular}{|l|l|l|l|l|}
\hline \multicolumn{5}{|c|}{ LED $=0.4 \mathrm{~J} / \mathrm{mm}$} \\
\hline Power (W) & 100 & 200 & 300 & 400 \\
\hline Scan speed (mm/s) & 250 & 500 & 750 & 1000 \\
\hline
\end{tabular}

Figure 6.15 displays the keyhole shapes when the laser has moved $0.125 \mathrm{~mm}$, $0.25 \mathrm{~mm}, 0.375 \mathrm{~mm}, 0.5 \mathrm{~mm}, 0.675 \mathrm{~mm}$ and $0.75 \mathrm{~mm}$. The respective time, which would vary with scan speed, has been shown in the figure. The behavior of the keyhole changes with a change in process parameters. For lower power $(100 \mathrm{~W})$ and speed $(250$ $\mathrm{mm} / \mathrm{s}$ ), the keyhole opening is rather small as shown in Figure 6.15(a). The keyhole opening increased with increase in power and speed. For higher power $(400 \mathrm{~W})$ and speed $(1000 \mathrm{~mm} / \mathrm{s})$, the keyhole opening is larger in diameter and the closing force is not enough due to which keyhole remains open for longer time. In addition, the depth of the penetration is lower for lower power and speed. This behavior may be explained with the expression shown in equation 6.14 which shows that melt pool depth is more affected by the laser power than scan speed. The non-dimensional depth $\left(\delta^{*}\right)$ of the melt pool (ratio of depth to the beam diameter) takes the form [89]

$$
\delta^{*}=f n\left(\frac{A C P}{\rho h_{s} \sqrt{D v \sigma^{3}}}\right) \text {, }
$$


where $C$ is a constant with no dimensions, $h_{s}$ is enthalpy at melting. The $100 \mathrm{~W}$ laser power lead to very small vapor opening due to lower intensity while $400 \mathrm{~W}$ power leads to higher vapor opening and higher melt pool depth.

Besides, the interaction between evaporation pressure and surface tension are different for different laser power and scan speed. When the intensity is not enough, the equilibrium state is nonexistent, which represent the conduction mode of melting. As the intensity keeps on increasing, deep penetration is observed and after some point, there exist equilibrium states which decides the stability of the keyhole melt pool. The keyhole front wall becomes more inclined with increasing laser power and scan speed.
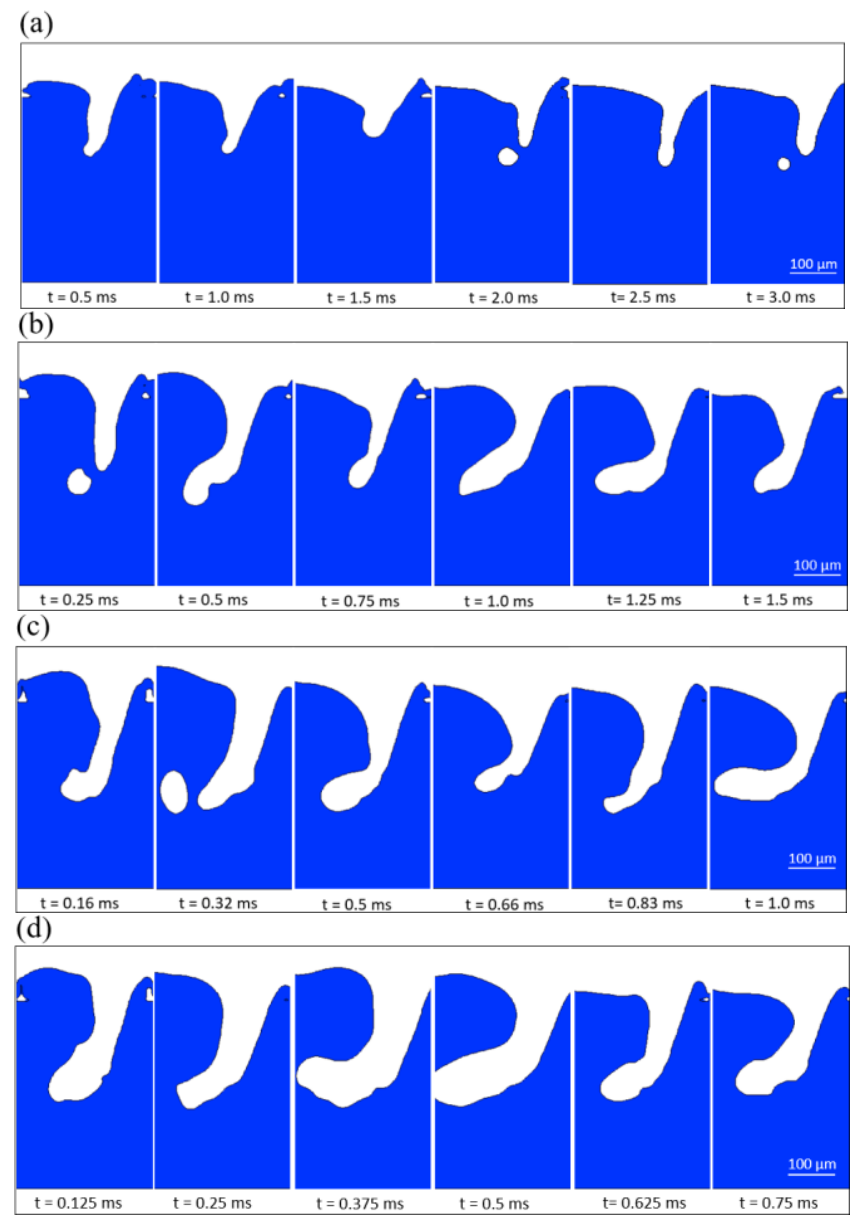

Figure 6.15 Keyhole shape at different time steps from different parameters, (a) $\mathrm{P}=100$ $\mathrm{W}, \mathrm{v}=250 \mathrm{~mm} / \mathrm{s}$, (b) $\mathrm{P}=200 \mathrm{~W}, \mathrm{v}=500 \mathrm{~mm} / \mathrm{s}$, (c) $\mathrm{P}=300 \mathrm{~W}, \mathrm{v}=750 \mathrm{~mm} / \mathrm{s}$ and (d) P $=400 \mathrm{~W}, \mathrm{v}=1000 \mathrm{~mm} / \mathrm{s}$. 


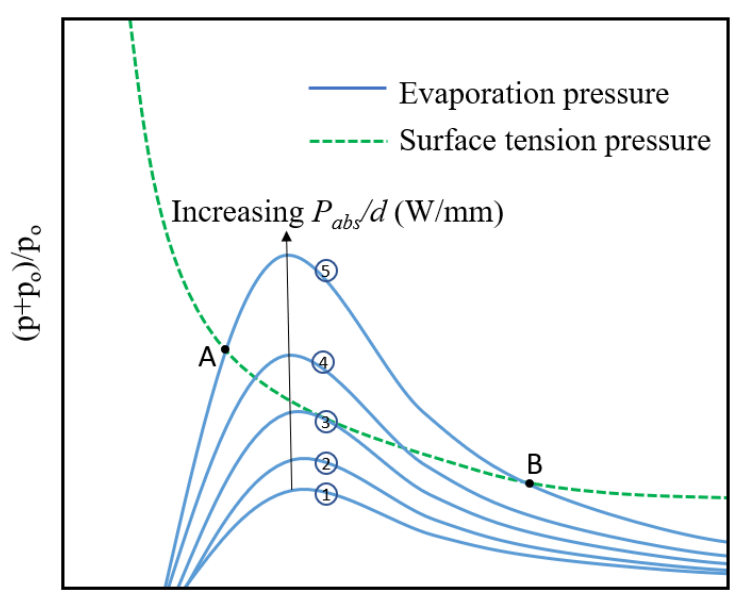

Normalized keyhole radius $(\mathrm{a} / \sigma)$

Figure 6.16 Intensity dependence in the relationship between vapor column and recoil pressure [20].

Figure 6.17 shows the temperature distribution during laser melting when power and speed of $300 \mathrm{~W}$ and $500 \mathrm{~mm} / \mathrm{s}$ and $400 \mathrm{~W}$ and $1000 \mathrm{~mm} / \mathrm{s}$ are used. The temperature of melt pool exceeds the evaporation temperature; therefore, the material evaporation occurs at the keyhole walls. However, the keyhole opening is different for these two cases. In this regard, the evaporation pressure effect for two cases would be different and hence the evaporation pressure and surface tension pressure interaction.

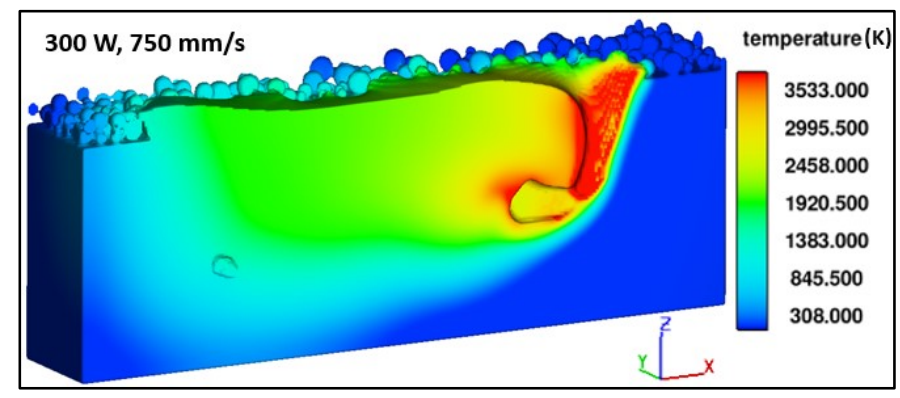




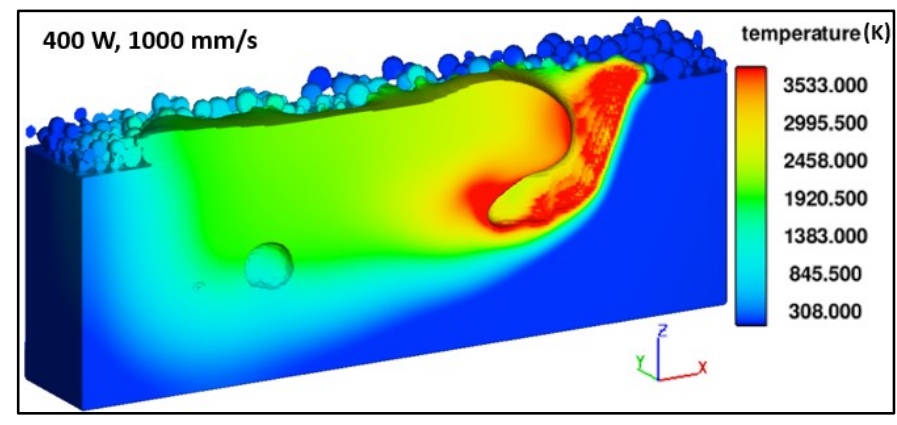

Figure 6.17 Temperature distribution when laser has moved $0.7 \mathrm{~mm}$ with $\mathrm{p}=100 \mathrm{w}, \mathrm{v}=$ $250 \mathrm{~mm} / \mathrm{s}$ and $\mathrm{p}=200 \mathrm{w}$ and $\mathrm{v}=500 \mathrm{~mm} / \mathrm{s}$.

Figure 6.18 exhibits the melt pool boundary formed after solidification. The pores trapped within the melt pool are also identified. The melt pool depth increased with increasing power from $100 \mathrm{~W}$ to $300 \mathrm{~W}$, however, there is a drop in the melt pool depth when the power is further increased from $300 \mathrm{~W}$ to $400 \mathrm{~W}$. This may be due to the change in absorptivity within the melt pool. At some point, when the vapor column exceeds the beam diameter, the absorptivity of the melt pool remains constant, which would explain the reduction of the melt pool depth as speed is also increased along with power. Trapp et al. [88] have performed an experiment to measure the absorptivity of metallic powders during the LPBF process. It was observed that the absorptivity of melt pool increases with the increase in power due to keyhole formation, however, the absorption finally saturates and remain constant on further increase in power. In addition, as the keyhole wall became more inclined with increasing power and speed, the final melt pool shape is also affected by this. 


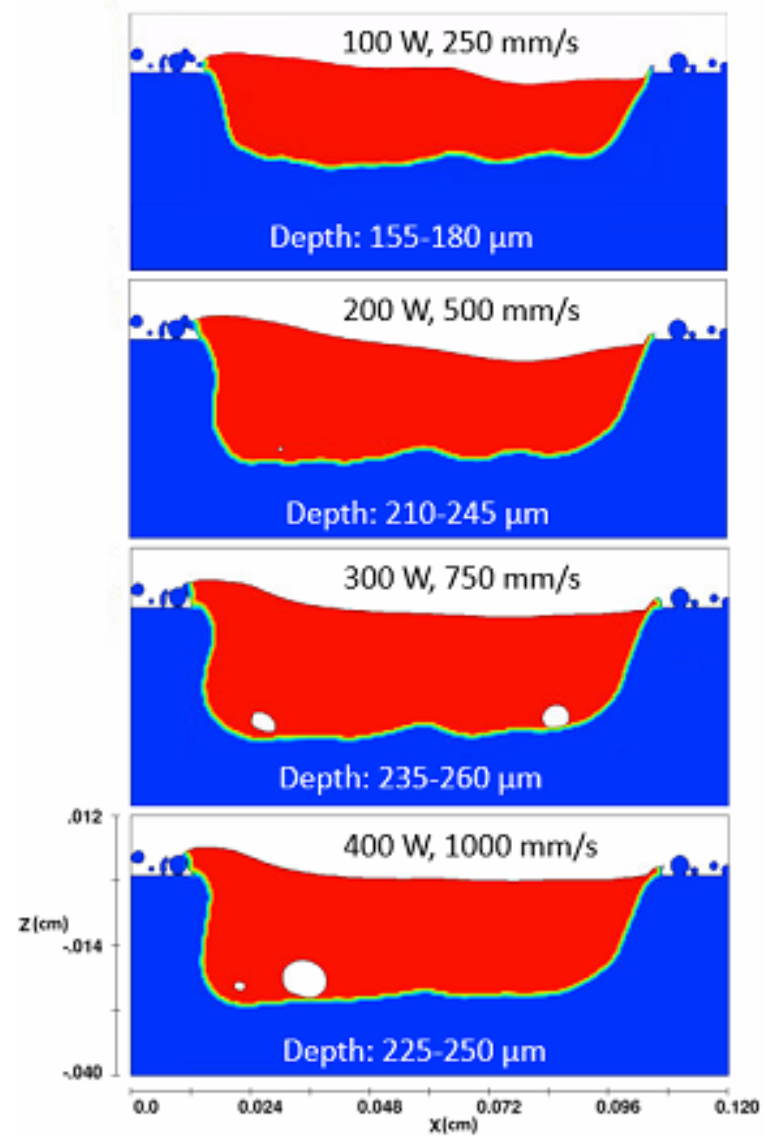

Figure 6.18 Longitudinal melt region obtained with different power and speed at same energy density.

\subsection{Conclusions}

A 3D thermo-fluid model has been developed to simulate the LPBF process and investigate the keyhole behavior and pore formation. DEM was used to simulate the particle distribution in a powder bed and the output was exported as a domain for the thermo-fluid simulation model. A moving Gaussian heat source, surface tension gradient, evaporation pressure along with multiple reflections within a keyhole were included to predict the melt pool and keyhole behavior. The melt pool results with both conduction and keyhole mode melting have been compared with the experiments from the literature; 
the melt pool shape and size from the transverse cross-section show a reasonable agreement. As to the keyhole formation in LPBF, its stability strongly depends on the process parameters used. Following are the major findings derived from this study:

- In conduction mode melting, the applied energy is lower with only small depression formed in the melt track. There is no significant interaction between the surface tension pressure and the evaporation pressure in such cases, and thus, no pores were observed.

- In keyhole mode melting, the radius of the keyhole is a strong function of the laser power, and therefore, the keyhole behavior changes with the laser power even at the same energy density.

- The keyhole depth increased with an increase in the laser power from $100 \mathrm{~W}$ to $300 \mathrm{~W}$, but then slightly decreased when the power was further increased from $300 \mathrm{~W}$ to $400 \mathrm{~W}$, possibly due to the saturation of the laser energy absorption within the keyhole. 


\section{CHAPTER 7 A STUDY OF PORE FORMATION DURING SINGLE LAYER AND MULTIPLE LAYER BUILD BY SELECTIVE LASER MELTING}

\subsection{Introduction}

Selective laser melting (SLM) is a powder bed additive manufacturing (AM) process which utilizes metal powder as feedstock material. Powder particles are added layer upon layer, and the selected area is melted with the laser. The layer by layer process enables the fabrication of complex components which are otherwise impossible to be fabricated with a subtractive technique. However, the process may form a different kind of defects such as porosities, part deformation, etc. These defects which are formed during the SLM process should be mitigated to improve the part performance.

Pores in the SLM process generally form due to process parameter settings. High energy density leads to keyhole pores, whereas the use of very low energy density leads to lack of fusion pores[5,65]. But the energy density may not be enough to characterize the porosity [106]. The track morphology, depth of the melt pool, and pore formation are highly dependent on power than scanning speed for the same energy density [106]. Besides, simulation study has shown that the keyhole pores are formed due to the instability of the keyhole, which depends on the applied power [107].

Keyhole pores and lack of fusion pores contribute differently towards the mechanical behavior of the components [35]. A small number of pores formed due to high energy input is harmless when present up to 1 vol.\% whereas the effect of the pore 
formed by insufficient energy is significant even if the amount is as low as 1 vol.\%. Kasperovich et al. [5] presented the correlation between the process parameters and the types of pores formed during SLM fabrication of Ti-6Al-4V parts. Generally, two types of void defects are present in SLM parts, circular/spherical pores are formed with the excessive energy input, whereas insufficient energy density results in elongated, narrow crack-like voids. Reducing these porosities improve tensile performance [108], fatigue performance [32,33], and hardness [31]. In addition, it is also important to characterize the pores, as the pore location and pore size are critical to the stress concentration and pores near to the surface are more critical even if they are small in size [34].

A few studies have focused solely on the pore formed during the SLM process. Ponnusamy et al. [6] carried out statistical analysis to investigate the effect of laser power, layer thickness, etc. on porosity. As pores are the internal features, X-ray based studies are suitable for non-destructive analysis of porosity and hold an advantage over destructive porosity analysis [38]. Maskery et al. [109] stressed the importance of CT based study as the statistical quality obtained by the data from CT would not be available my metallography due to the requirement of a large number of micrographs. But, the quality of the data obtained from CT analysis depends on its resolution. Siddique et al. [34] performed both X-ray based pore characterization using a voxel resolution of $4.8 \mu \mathrm{m}$ to scan the SLM specimen and two-dimensional metallographic study to calculate the porosity. The authors found that porosity calculated by metallographic studies and threedimensional tomography had no significant difference considering the critical pores. Similarly, Wits et al. [110] explained the difference in pore size measurement between micrograph and CT study. The CT always measured the smaller area, but both optical 
techniques and CT are influenced by several factors which could lead to the observed diversity. Slotwinski et al. [7] utilized X-ray computed tomography (XRCT) to measure porosity on additively manufactured cobalt-chrome (Co-Cr) samples which helped determine the pore distribution within the sample as well as the pore morphology. In a similar manner, Zhou et al. [37] used Synchrotron radiation micro-CT to observe the defects formed within SLM parts. The authors concluded that micro-CT imaging technique is a feasible method to obtain 3D images of SLM defects accurately. Kim et al. [39] utilized $\mu \mathrm{CT}$ as a feedback mechanism to make improvements on initial designs and Van Bael et al. [40] used XRCT as a feedback mechanism to improve the geometrical and mechanical controllability of selective laser melted Ti-6Al-4V porous structures.

This study uses previous single-track results [106] to design the experiment and investigate the effect of hatch spacing. Hatch spacing is another variable, besides laser power and scan speed, which contribute towards the volumetric energy density. Hatch spacing affects the maximum temperature and heat accumulation [111]. Track variations depending upon the hatch spacing is the inherent phenomena of SLM process[112]. Thijs et al. [113] used hatch spacings of $50 \mu \mathrm{m}, 70 \mu \mathrm{m}$ and $100 \mu \mathrm{m}$, while the width of the single track was $97 \mu \mathrm{m}$. The micrograph results showed that small pores are formed with smaller hatch spacing, while with $100 \mu \mathrm{m}$ hatch spacing, larger elongated pores are formed. Read et al. [83] performed analysis of variance to investigate the individual and interaction effect of laser power, scan speed and hatch spacing on the porosity. Based on the analysis, hatch spacing alone did not affect the porosity, but the interaction between the scan speed and hatch spacing affected the porosity. The combinations of laser power $(125 \mathrm{~W}, 150 \mathrm{~W}, 175 \mathrm{~W}$, and $195 \mathrm{~W})$ and scan speed $(600 \mathrm{~mm} / \mathrm{s}, 800 \mathrm{~mm} / \mathrm{s}, 1000 \mathrm{~mm} / \mathrm{s}$ 
and $1200 \mathrm{~mm} / \mathrm{s}$ ) from conduction regime are selected and hatch spacing is calculated based on the percentage of the measured track widths. Six levels of hatch spacings are investigated: $60 \%, 70 \%, 80 \%, 90 \%, 120 \%$ and $150 \%$ of track width. Again, micro-CT is used to scan the samples and observe the interior pores. Total pore numbers and volume obtained due to the different parameter settings are compared.

\subsection{Approach}

\subsubsection{Design of Experiment}

This study is focused on the effect of hatch spacing on the porosity during the SLM process. The single track experiment was performed at first [106], and the measured track width results are utilized to design the single layer and multilayer experiments. Figure 7.1 shows a typical example of single-track transverse profile showing the width of the single track. The top bead is identified with $\mathrm{CT}$ analysis which enables the measurement of track width, even without the observation of the melt profile formed beneath the surface. Hatch spacing is the gap between two successive laser tracks as shown in Figure 7.1. A percentage of the single-track width is used to define the hatch spacing and $60 \%, 70 \%, 80 \%, 90 \%, 120 \%$, and $150 \%$ of the track widths are used to form

single layer and multilayers. Table 7.1 lists the laser power, laser speed, track widths from different combination of power and speed, and the corresponding hatch spacing. 


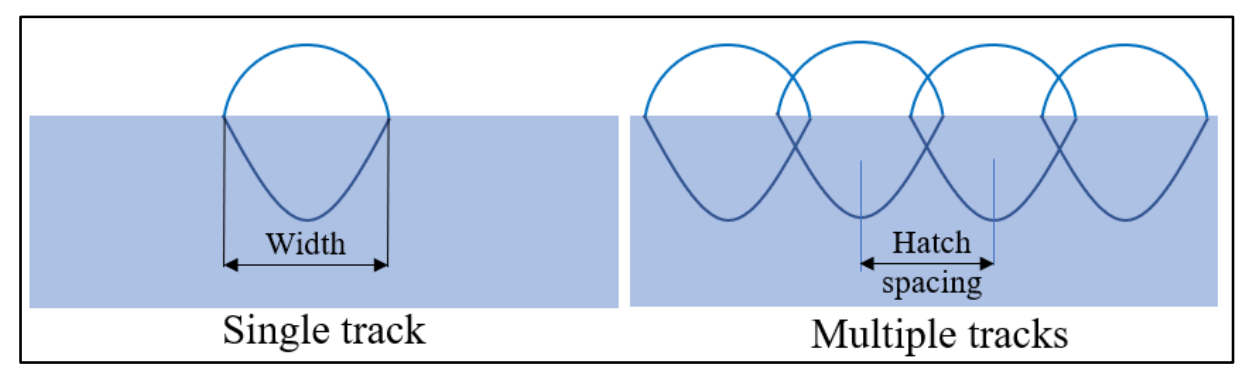

Figure 7.1 Single track geometry and hatch spacing definition in single layer scanning.

Table 7.1 Process parameters used in single layer and multilayer design.

\begin{tabular}{|l|l|l|l|l|l|l|l|l|}
\hline \multirow{2}{*}{$\begin{array}{l}\text { Laser } \\
\text { power }(\mathrm{W})\end{array}$} & \multirow{2}{*}{$\begin{array}{l}\text { Scan speed } \\
(\mathrm{mm} / \mathrm{s})\end{array}$} & \multirow{2}{*}{$\begin{array}{c}\text { Track } \\
\text { width }(\mu \mathrm{m})\end{array}$} & \multicolumn{6}{|c|}{ Hatch spacing $(\mu \mathrm{m})$} \\
\cline { 5 - 9 } & & 600 & 138 & $80 \%$ & $80 \%$ & $90 \%$ & $120 \%$ & $150 \%$ \\
\hline 125 & 600 & 120 & 72 & 84 & 96 & 108 & 144 & 180 \\
\hline 125 & 800 & 101 & 61 & 71 & 81 & 92 & 122 & 152 \\
\hline 125 & 1000 & 145 & 87 & 102 & 116 & 130 & 174 & 217 \\
\hline 150 & 600 & 133 & 80 & 94 & 107 & 120 & 160 & 200 \\
\hline 150 & 800 & 121 & 73 & 85 & 97 & 110 & 146 & 182 \\
\hline 150 & 1000 & 143 & 86 & 100 & 115 & 129 & 172 & 215 \\
\hline 175 & 800 & 140 & 84 & 98 & 112 & 126 & 168 & 210 \\
\hline 175 & 1000 & 127 & 76 & 91 & 103 & 114 & 152 & 190 \\
\hline 175 & 1200 & 163 & 98 & 114 & 131 & 147 & 196 & 245 \\
\hline 195 & 800 & 155 & 93 & 109 & 125 & 140 & 186 & 232 \\
\hline 195 & 1000 & 135 & 81 & 94 & 108 & 121 & 162 & 202 \\
\hline 195 & 1200 & & & & & & & 166 \\
\hline
\end{tabular}

\subsubsection{Sample Design and Fabrication}

Figure 7.2(a) shows the cylinder design dimensions of the Ti-6Al-4V samples fabricated with EOS M270. The external length and diameter of the cylinder are $18 \mathrm{~mm}$ and $7 \mathrm{~mm}$, respectively. The micro-CT can scan $13 \mathrm{~mm}$ length when using $6 \mu \mathrm{m}$ pixel size, hence two notches are designed to identify the region containing the test subjects. Each cylinder contains three $3 \mathrm{~mm} \times 3 \mathrm{~mm}$ area inside. These areas are formed with 
different level of hatch spacings. Figure 7.2(b) shows the cylinder samples after the supports were removed. Figure 7.3 presents the scan pattern for the single layer and multi-layer builds. For a single layer, X-direction raster scanning is performed, while for multi-layer, alternate $\mathrm{X}-\mathrm{Y}$ raster scanning is performed. 33 layers with each layer thickness of $30 \mu \mathrm{m}$ were formed to obtain the multi-layer sample.

(a)

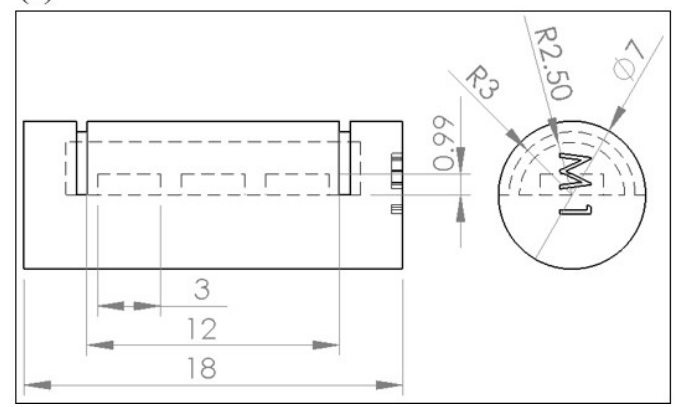

(b)

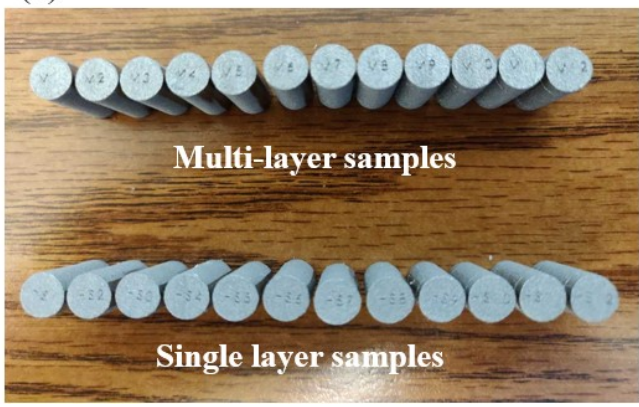

Figure 7.2 (a) Sample design dimensions and (b) Single layer and multi-layer samples fabricated by EOS M270.
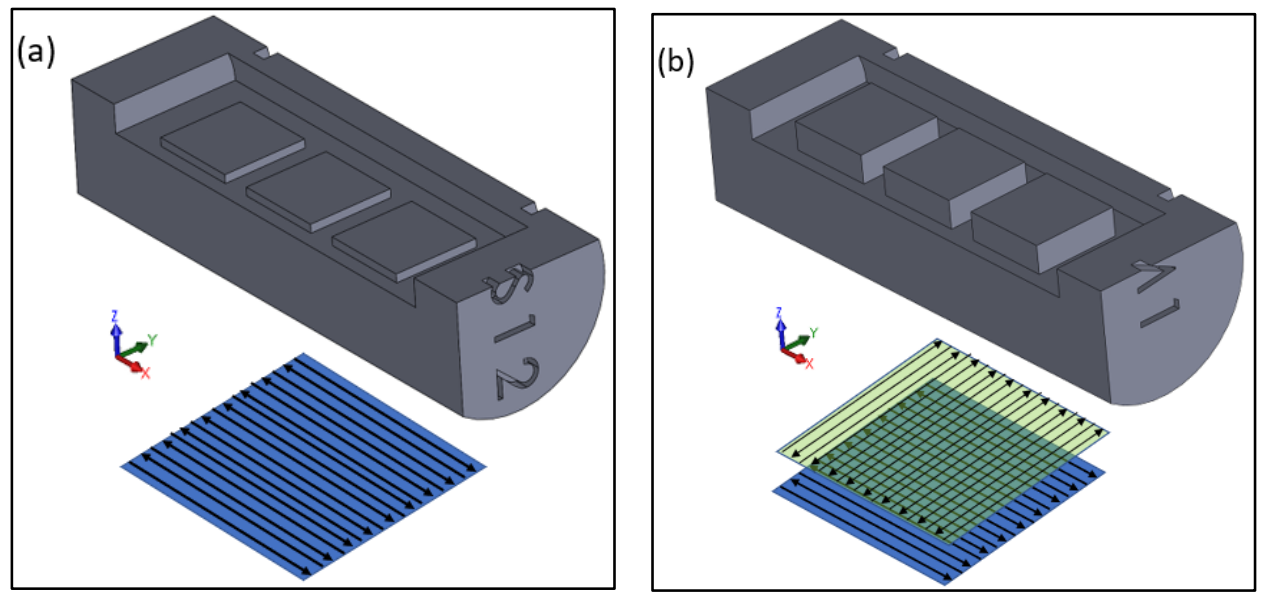

Figure 7.3 (a) single layer and (b) multi-layer design along with the scan strategies. 


\subsection{Results and Discussion}

\subsubsection{Typical scanning images}

The single layer and multilayer samples were scanned using a Bruker 1173 microCT scanner, and the data reconstruction and pore analysis explained in the reference [106] is performed. Figure 7.4 presents the sectional views of single layer sample formed with $150 \mathrm{~W}$ and $600 \mathrm{~mm} / \mathrm{s}$ at different hatch spacings. Three areas on top of the base pad are clearly observed, and the overlap of the track is different for different hatch spacing conditions. Three regions can be identified from the grayscale image: scanned region (light gray), powder region (dark gray) and voids (black). The sectional views of multilayers, formed with $175 \mathrm{~W}$ and $800 \mathrm{~mm} / \mathrm{s}$ with different hatch spacing of $86 \mu \mathrm{m}, 100 \mu \mathrm{m}$, and $115 \mu \mathrm{m}$, is also shown in Figure 7.4.

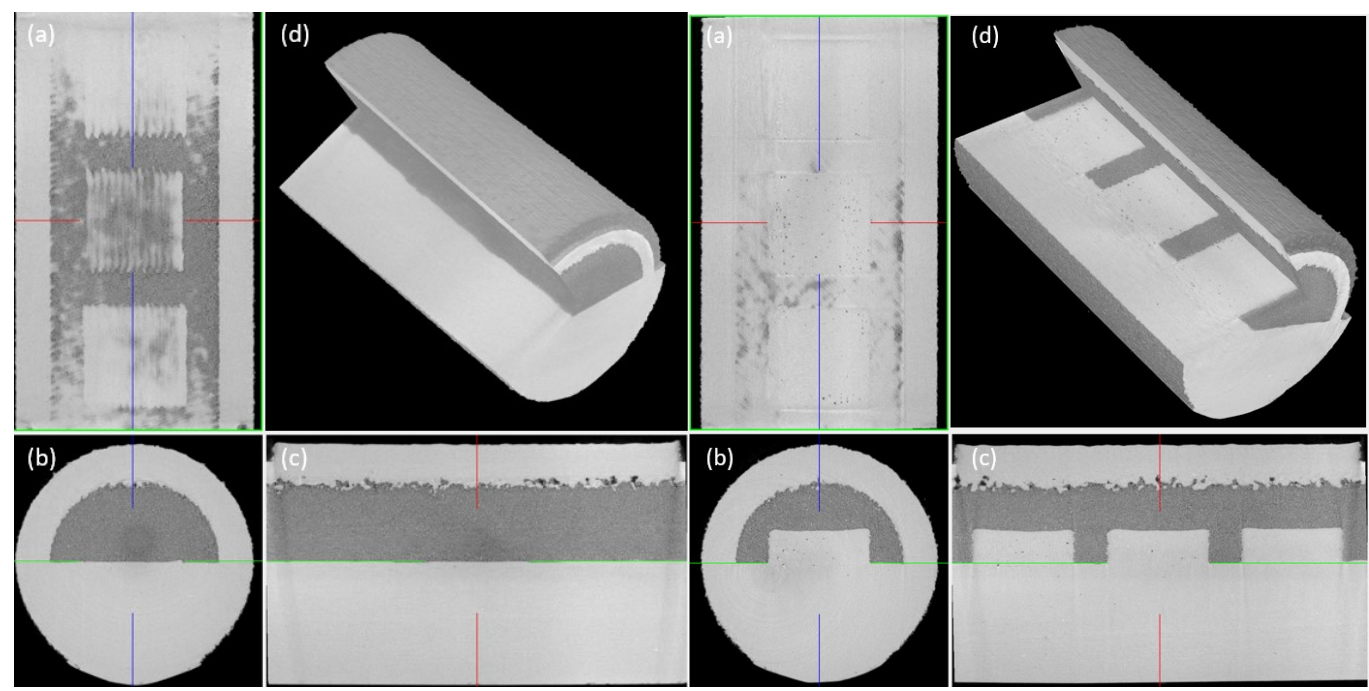

Figure 7.4 (a) Coronal (X-Z), (b) Transaxial (X-Y), (c) Sagittal (Z-Y) cross-sectional views and (d) partial cut-off view of a single layer and multi-layer. 


\subsubsection{Single layer experiment results}

The single layer surface morphology is shown in Figure 7.5. The surface is smooth for lower hatch spacing, and the single tracks become more distinct with an increase in hatch spacing. Figure 7.6 shows the pores formed due to the parameter settings. The parameters were selected based on single track experiment. The average number of pores obtained from three $12 \mathrm{~mm}$ long single tracks formed with $125 \mathrm{~W}$ and $1000 \mathrm{~mm} / \mathrm{s}$ is only 0.33 , that is one pore was observed in one of the tracks, and two of the tracks did not show any pore. Hence, very few pores are observed in single layer experiment too, as only the pores which are formed below the surface is analyzed and no surface pores were analyzed.
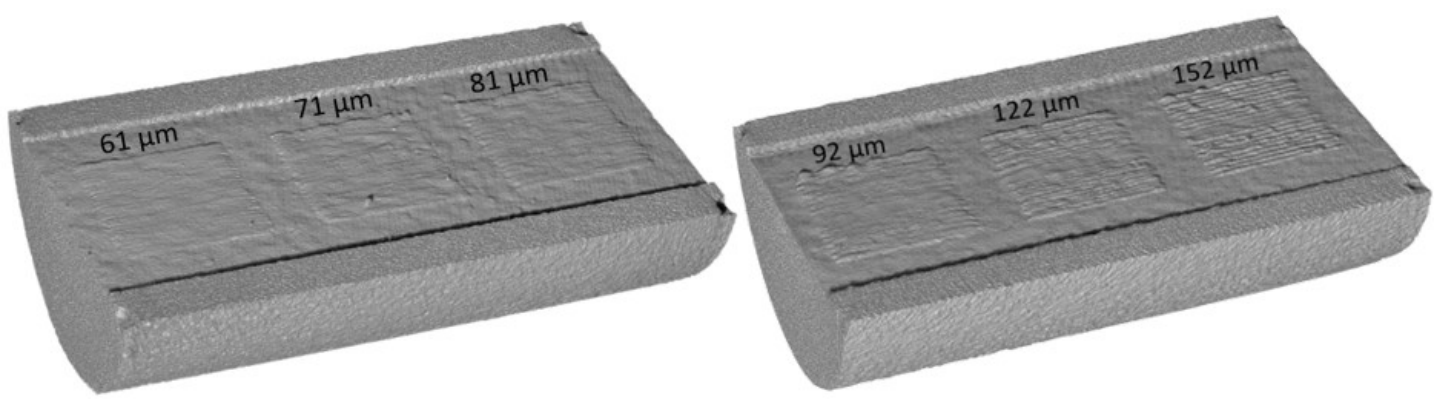

Figure 7.5 Surface morphologies due to different hatch spacing at $125 \mathrm{~W}$ power and 1000 $\mathrm{mm} / \mathrm{s}$ scan speed.
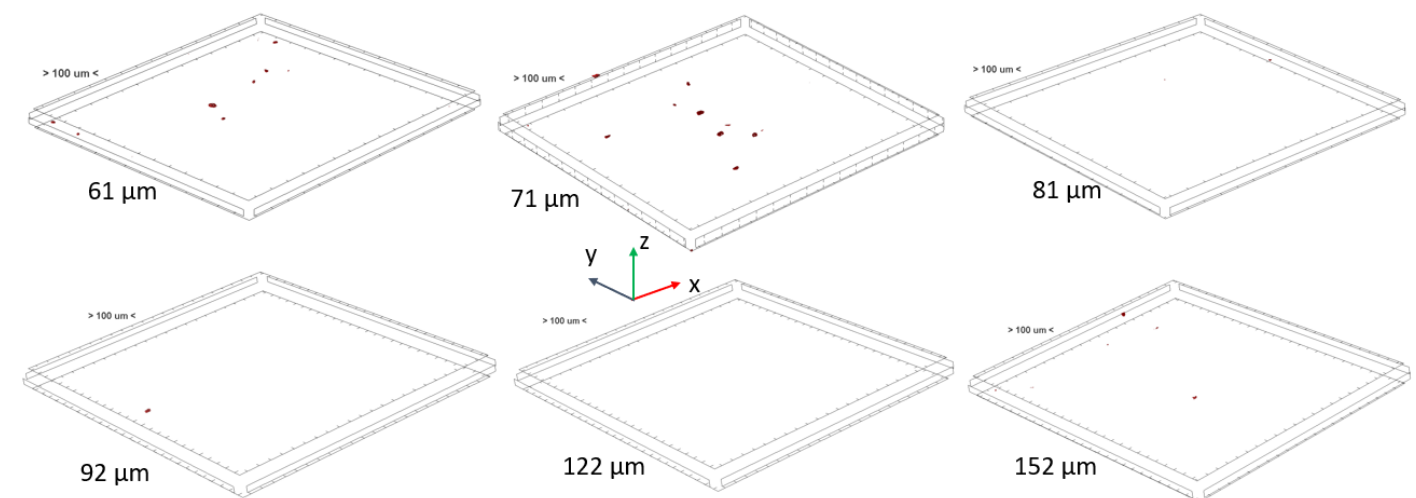

Figure 7.6 Pore formed due to different hatch spacing at $125 \mathrm{~W}$ power and $1000 \mathrm{~mm} / \mathrm{s}$ scan speed. 
Figure 7.7 shows the pore number obtained from single layers formed with different laser power, scan speed and hatch spacings. Only one sample for each case is analyzed for single-layer samples. No distinct trend is observed from the single layer experiment, mostly due to the limited number of pores being formed. $150 \mathrm{~W}$ and $600 \mathrm{~m} / \mathrm{s}$, however, resulted in significantly higher number of pores for all hatch spacing compared to other parameters. This may be due to keyhole regime of melting with these parameters. When the pore number is higher, a trend can be observed, that is with increasing hatch spacing, the number of pores mostly decreased. For other cases, hatch spacing did not seem to affect the number of pores during the single layer formation. Besides, the pores may also form due to several other factors like powder spatter formation, etc. which have not been considered in this study.

Higher hatch spacing may lead to lack of fusion pores. Due to the single layer formation, the lack of fusion pores is difficult to identify based on the surface quality. Hence, multilayer fabrication is important to investigate the effect of hatch spacing on the formation of lack of fusion porosity. In addition, $150 \mathrm{~W}$ and $600 \mathrm{~mm} / \mathrm{s}$ case demonstrated that higher number of pores may show some relationship between hatch spacing and the porosity level. The multilayer build is expected to form higher number of pores compared to single layer pores which would help observe the effect of hatch spacing more evidently. 

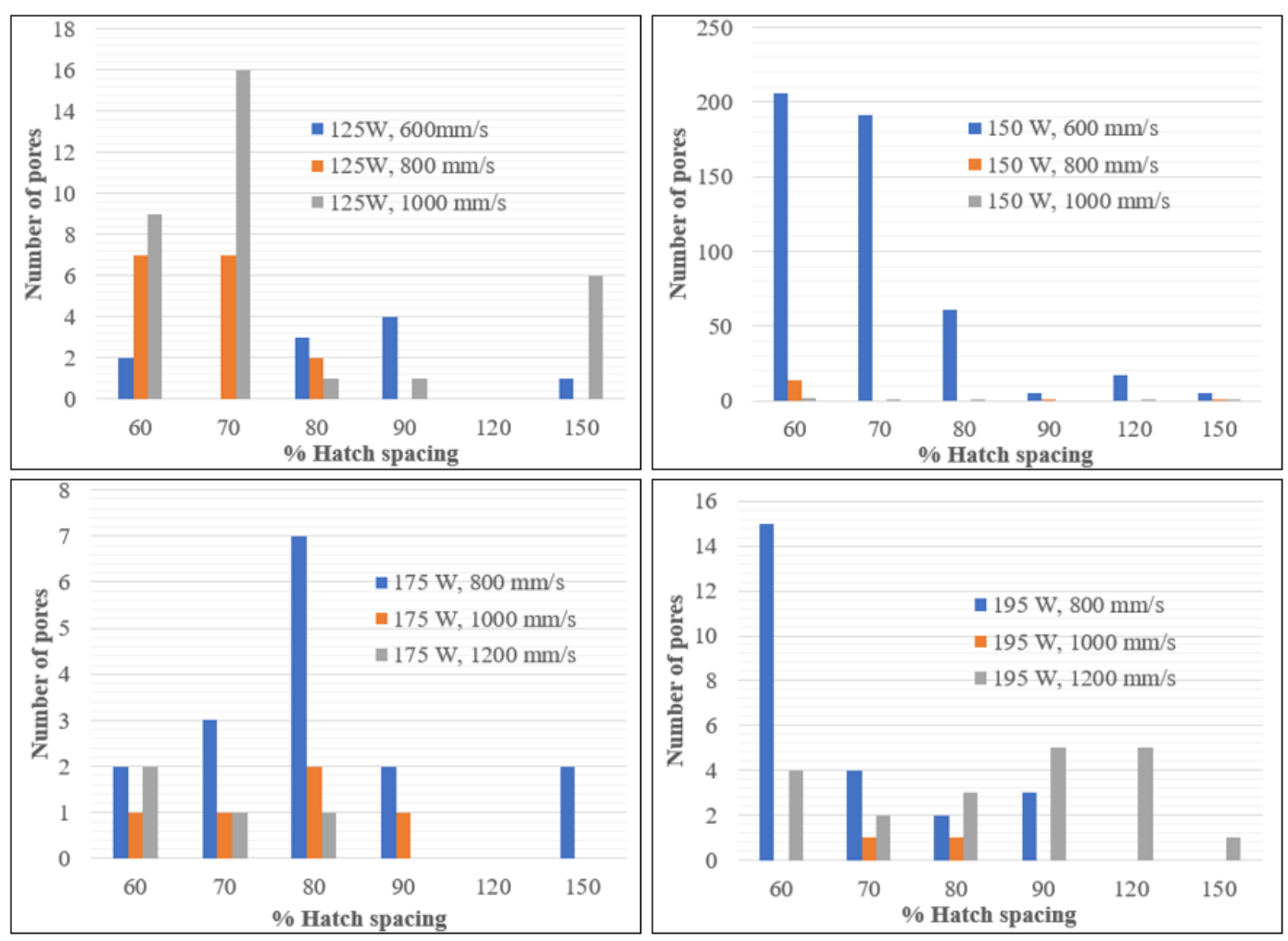

Figure 7.7 Pore number formed within single layer with different laser parameters

\subsubsection{Multiple layer experiment results}

The multilayer part formed are shown in Figure 7.8. The surface with lower hatch spacing has smoother surface which is due to the re-melting of the curved surface formed during single track formation. But, with increase in hatch spacing, the re-melting is insufficient and wavy surfaces are observed. For the hatch spacings greater than track width, individual tracks are identified. Figure 7.9 shows the pore distribution when $120 \%$ and $150 \%$ hatch spacings are used. A higher number of pores are observed for higher hatch spacing compared to the hatch spacing lower than $100 \%$. The increase in porosity with increase in hatch spacing may be due to the lack of fusion between the tracks. As the hatch spacing of $150 \%$ formed a mesh like structure, due to insufficient bonding between the tracks, it is not included in the analysis. 


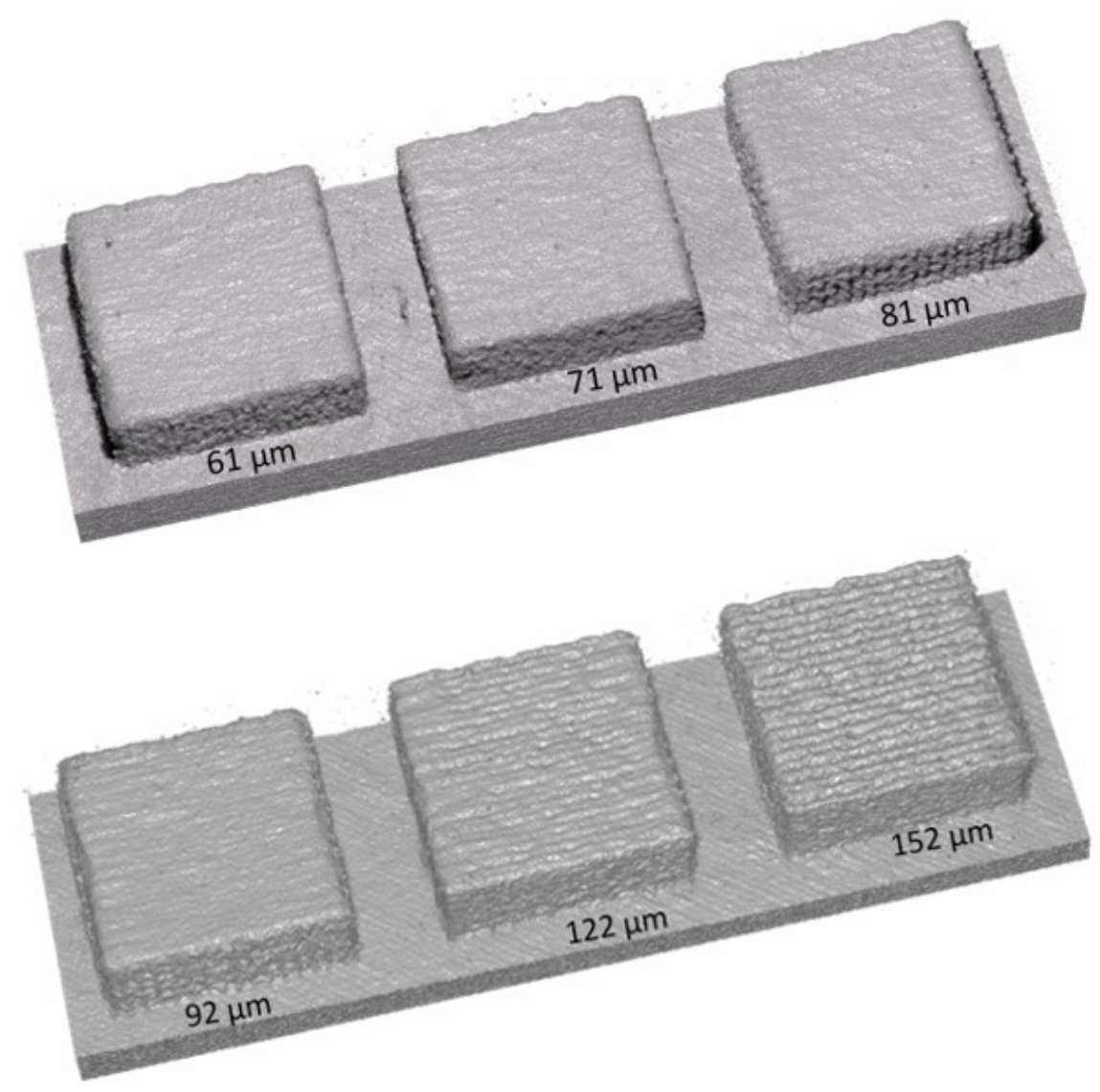

Figure 7.8 Surface morphologies due to different hatch spacing at $125 \mathrm{~W}$ power and 1000 $\mathrm{mm} / \mathrm{s}$ scan speed.
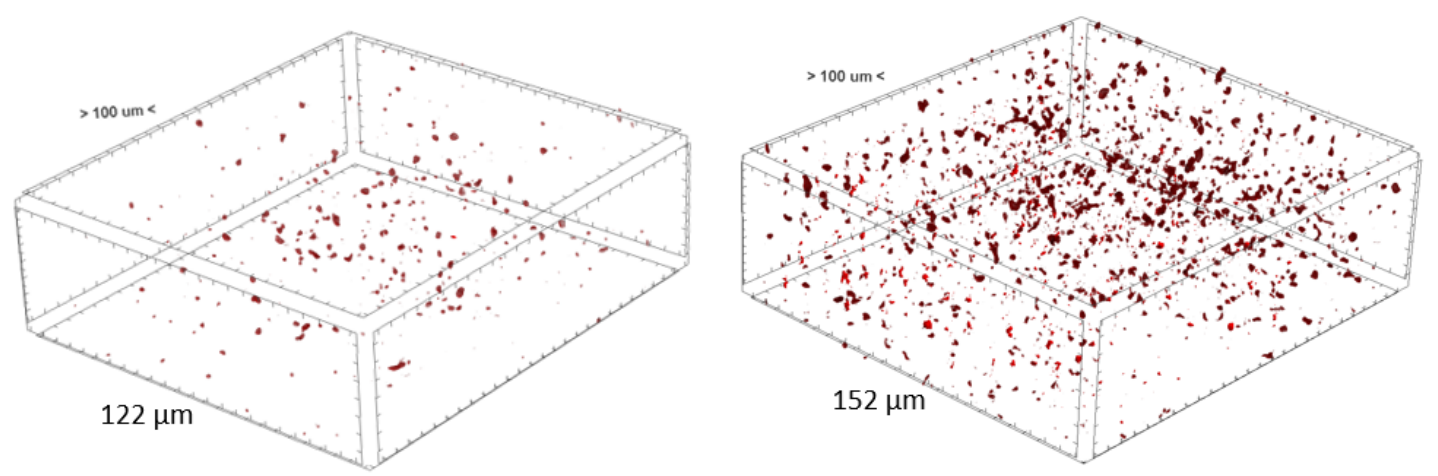

Figure 7.9 Pores formed due to hatch spacing of $122 \mu \mathrm{m}$ and $152 \mu \mathrm{m}$ at $125 \mathrm{~W}$ and 1000 $\mathrm{mm} / \mathrm{s}$.

Figure 7.10 presents the average number of pores formed, and standard deviation from three replicates, within the multilayer formed with different process parameters. In general, the pore number decreased with increasing hatch spacing up to $90 \%$, while the 
pore number increased drastically from $90 \%$ to $120 \%$. But, for $195 \mathrm{~W}$, the porosity was minimum when $80 \%$ hatch spacing is used. Also, the pore number decreased with decrease in energy density, that is increase in speed for same power and hatch spacing. Although lower hatch spacing resulted in smoother surface, the higher overlap resulted in higher number of pores. When the hatch spacing is lower, the residual heat may affect the melt pool and at some region keyhole may form resulting to the formation of pores. As the hatch spacing increased from $90 \%$ to $120 \%$, the pore number increased significantly. This may be primarily due to the insufficient overlap between the tracks leading to the formation of lack of fusion pores. Figure 7.9 showed that many elongated pores are formed when $120 \%$ hatch spacing is used except for $150 \mathrm{~W}$ and $600 \mathrm{~mm} / \mathrm{s}$. This may be due to the variation in track widths formed during successive scanning compared to the single track. During the single-track experiment, only one track is formed, while during the area scanning, residual heat may build up due to successive scanning, leading to wider tracks.

Among the parameters tested, $150 \mathrm{~W}$ and $600 \mathrm{~mm} / \mathrm{s}$ resulted in significantly higher number of pores. This is expected as single layer results also show that these parameters lead to higher number of keyhole pores. However, in this case, $120 \%$ hatch spacing resulted in fewer number of pores compared to lower hatch spacing, unlike all other set of parameters. Based on the results, porosity can be significantly reduced with proper selection of process parameters for example, $150 \mathrm{~W}, 1000 \mathrm{~mm} / \mathrm{s}$ and $90 \%(110$ $\mu \mathrm{m})$ hatch spacing. 


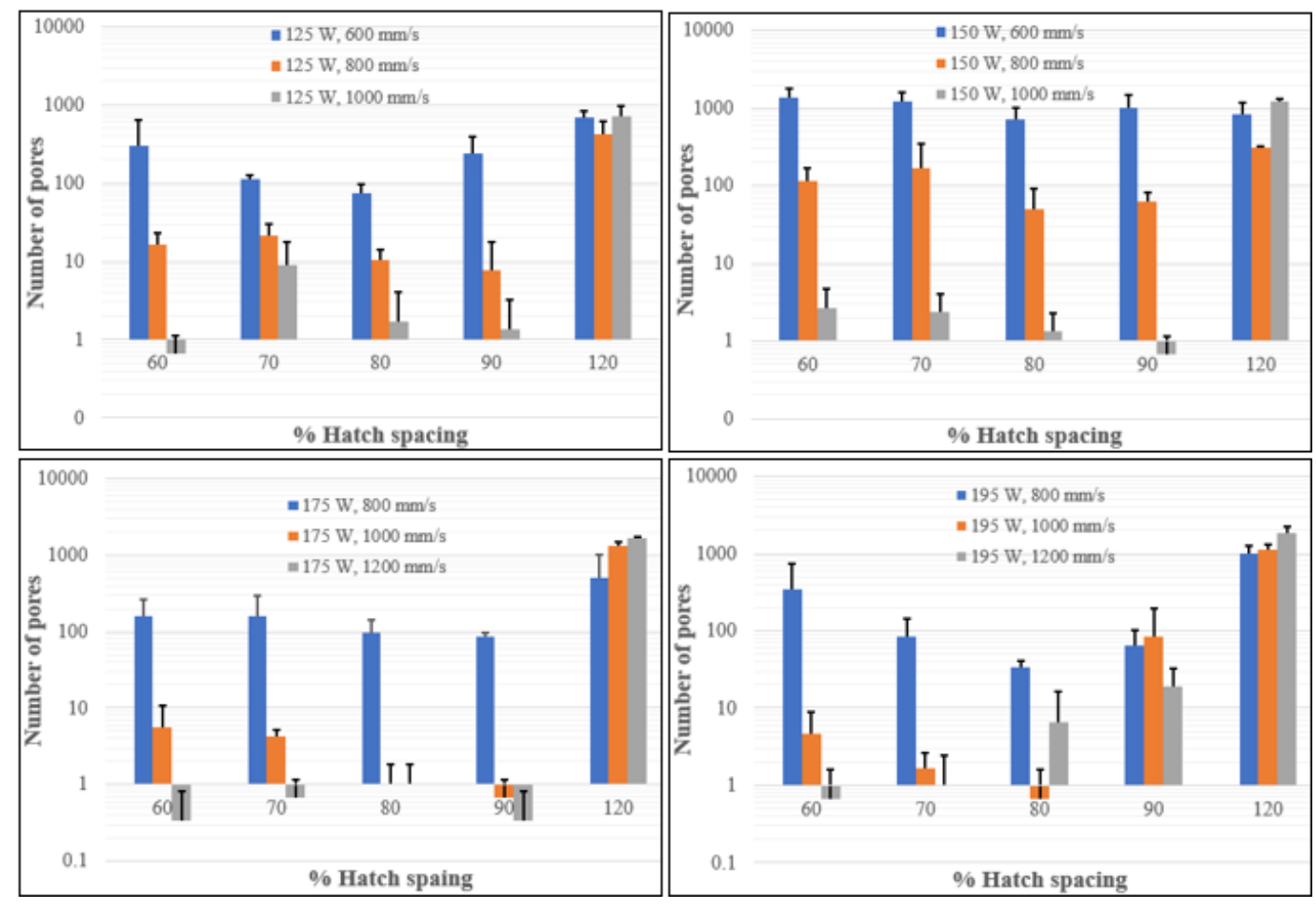

Figure 7.10 Number of pores formed at different levels of hatch spacing.

The volume equivalent diameter of pores is measured and the summary of the average, minimum and maximum pore diameter is presented in Figure 7.11. There is negligible variation in the average, minimum and maximum pore diameter with respect to hatch spacing for the same power and scan speed. Hence it is observed that the hatch spacing does not affect the size of the keyhole pores. The average pore size decreased with increase in speed for same power as observed in the single-track experiment. $150 \mathrm{~W}$ and $1000 \mathrm{~mm} / \mathrm{s}$ formed negligible or no pore at $80 \%$ and $90 \%$ hatch spacing. For $150 \mathrm{~W}$, $600 \mathrm{~mm} / \mathrm{s}$ and $150 \mathrm{~W}, 800 \mathrm{~mm} / \mathrm{s}$, no obvious change in the pore size is observed when hatch spacing increased from $90 \%$ to $120 \%$. But, for $150 \mathrm{~W}$ and $1000 \mathrm{~mm} / \mathrm{s}$, the average pore size increased, and the maximum pore size measured is $77 \mu \mathrm{m}$. As the hatch spacing 
increased, elongated voids may generate due to the insufficient or no overlap between the tracks.

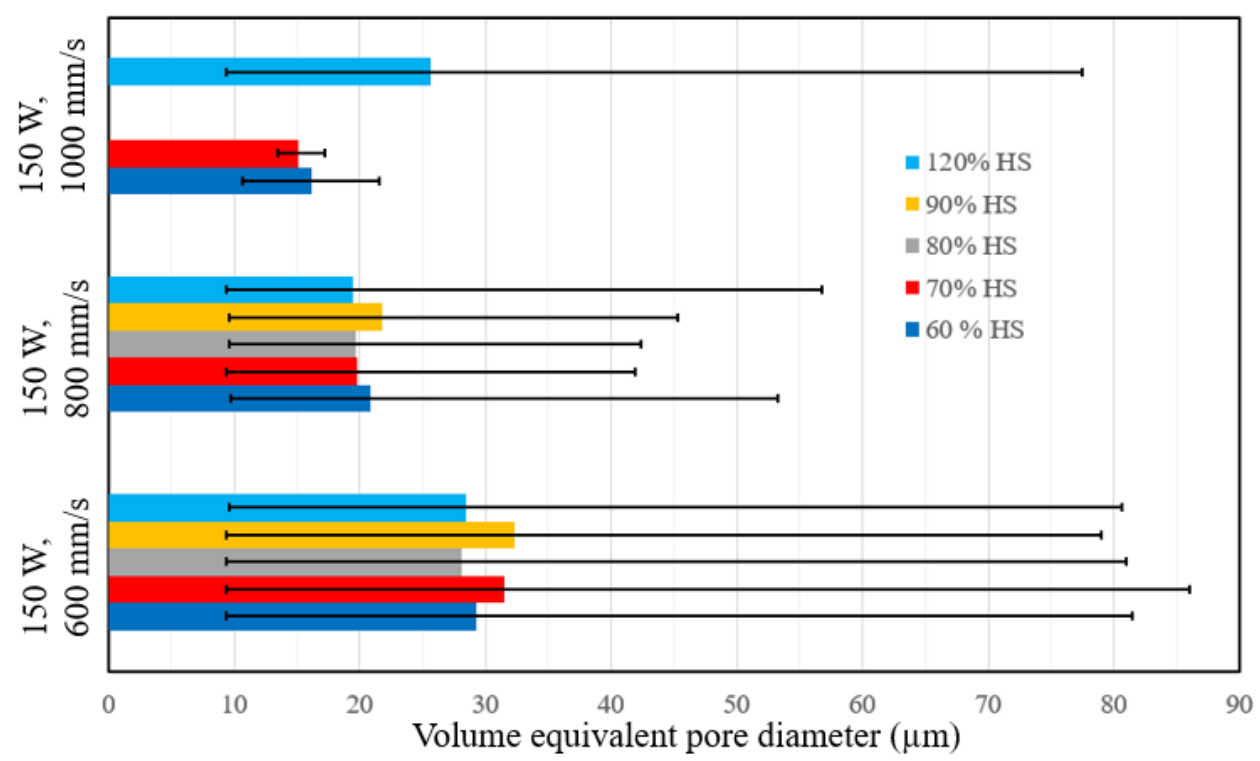

Figure 7.11 Average pore diameter formed with $150 \mathrm{~W}$ at different scan speed and hatch spacings.

\subsection{Conclusions}

In this study, single layer and multilayer samples are fabricated with Ti-6Al-4V using EOS M270 to investigate the effect of hatch spacing for different combination of laser power and scan speeds. Three cuboids of $3 \mathrm{~mm} \times 3 \mathrm{~mm} \times 0.99 \mathrm{~mm}$ were fabricated within each cylinder sample, and the cylinder samples were scanned using Skyscan 1173 micro-CT scanner to measure the pores formed within. Five levels of hatch spacing, $60 \%$, $70 \%, 80 \%, 90 \%$, and $120 \%$ of track widths, were analyzed which led to the following conclusions.

- There is no clear effect of hatch spacing on the single-layer build. Less than 10 pores are formed in most of the cases during $3 \mathrm{~mm} \times 3 \mathrm{~mm}$ area scanning. 
- Multilayer porosity results show that the pore number depend on hatch spacing. The number of pores reduced with increasing hatch spacing up to from $60 \%$ to $90 \%$. But the increase in hatch spacing from $90 \%$ to $120 \%$ introduced lack of fusion pores which significantly increased the pore number.

- The hatch spacing of $60 \%$ to $90 \%$ did not affect the pore size, while the hatch spacing of $120 \%$ introduced lack of fusion pores which increased the average pore diameter. 
CHAPTER 8 AN INVESTIGATION INTO MELTING MODES IN SELECTIVE LASER MELTING OF INCONEL 625 POWDER: SINGLE TRACK GEOMETRY AND POROSITY

\subsection{Introduction}

Additive manufacturing (AM) is a layer by layer fabrication process which enables the production of complex geometries. Selective laser melting (SLM) is a powder bed AM process that uses metal powder as the feedstock material. During the SLM process, the metal powder is spread over the build platform layer by layer, and the laser is used to melt the powder particles selectively to form a designed part. SLM process has been used to produce parts from different materials such as titanium alloy $[33,114]$, steel $[61,75,115]$, nickel-based alloys [116, 117], etc.

Although the SLM process has shown its freeform capability, the variability induced by the process itself impedes the establishment of a process standard [118]. The process variables include powder size distribution, laser power, scan speed, hatch spacing, layer thickness, ambient pressure, inert atmosphere, etc.[119] Lee et. al [120] have used a data analytics approach to find the correlation between different parameters with the melt pool properties such as track width, depth, etc. The results showed that the depth, width, and the height of the melt pool are physically relevant with the laser penetration, fluid convection, and fluid properties of melted powders, respectively. 
The single-track studies are mostly used to observe the track morphology and the melt pool boundary $[62,82,87]$. These studies use a transverse melt pool boundary to distinguish the conduction mode melting and the keyhole mode melting. The shape of the melt pool boundary formed in the conduction mode is usually semi-circular, and the depth is close to the half-width of the track. However, the depth is higher than the halfwidth of the track in the keyhole mode melting [36]. Cunningham et. al [95] used ultrahigh-speed synchrotron x-ray imaging to quantify the melt pool formation during the SLM process. Such experiments enable the real-time observation of the development of vapor depression. The authors concluded that there is a well-defined threshold from conduction mode to keyhole based on laser power density. Similarly, Martin et.al [121] studied the dynamics of the pore formation during the SLM process. In-situ X-ray imaging was utilized to understand the formation of vapor depression, and the effect of laser power on the depression depth was assessed. The results indicated that under steady-state scan conditions, the vapor depression increases linearly with the laser power, and the pores are formed due to the collapse of the keyhole wall. Besides, the formation of the melt profile and the characteristics of the conduction mode melting and keyhole mode melting can also be understood through numerical simulations [107].

During the keyhole mode melting, the material evaporation exerts a recoil pressure, which leads to the formation of a keyhole. The instability in the keyhole walls may lead to the formation of the pores [85]. Micro-computed tomography (micro-CT) based studies are performed to characterize pores formed within the single tracks due to different linear energy densities $[106,122]$. The size of the keyhole pores depends on the process parameter used, and the keyhole porosity increase with the increase in energy 
density. Most of the studies used the energy density, a thermodynamic quantity, to characterize the single tracks and part quality [84]. But the melt pool characteristics in the laser material processing depend on the process parameters such as laser power, scan speed, and beam diameter, and material properties such as surface absorption, thermal conductivity, and thermal diffusivity [123]. Hence, it is important to include material properties while analyzing the track property. Hann et. al [89] incorporated the effect of the material properties and suggested that the weld profiles in the laser welding are related to the peak enthalpy of the weld pool.

In this study, EOS M270 is used to fabricate the IN625 single track samples and investigate the effect of linear energy density (LED) on the track property and porosity. A micro-CT scanner is used to measure and characterize the pores formed with different LEDs. Besides, the samples are sectioned, polished, and etched to reveal the melt pool boundary. The effect of LED on the melt pool boundary and the transition of the melting mode with the change in LED is observed. Also, the effect of power and speed on the melt pool depth is studied by keeping the LED constant at $0.48 \mathrm{~J} / \mathrm{mm}$ and $0.75 \mathrm{~J} / \mathrm{mm}$. Again, micro-CT is used to characterize the pores, and metallography is performed to reveal the melt pool boundary. Moreover, the normalized enthalpy is utilized to characterize the transition of the melt pool from the conduction mode to the keyhole mode. Further, the powder scale numerical model is used to investigate the heat transfer and melt flow behavior at different power and speed combinations from the same LED. A small-scale model is developed to understand the underlying physics behind the change of melting mode from conduction to keyhole. 


\subsection{Experiment Approach}

Figure 8.1(a) presents the design and dimensions of hollow cylinder samples. The cylinder design is implemented to aid the CT scanning. Besides, it is necessary to ensure adequate $\mathrm{X}$-ray transmission (130 kV source) through the sample. As the density of IN625 $(8440 \mathrm{Kg} / \mathrm{m} 3)$ is high, the diameter of $3 \mathrm{~mm}$ is used in this study to allow proper transmission. Figure 8.1(b) shows the arrangement of the single tracks on top of the semicylinder base. The $12 \mathrm{~mm}$ tracks are formed between the notches, which works as a reference, and is used to locate the tracks during the CT scanning. The tracks are formed with a $0.7 \mathrm{~mm}$ gap to minimize the effect of denudation from one track to another[69]. EOS M270 is used to fabricate the single-track samples with $40 \mu \mathrm{m}$ layer thickness in this study. The tracks are formed with the user parameters, while the hollow cylinder is fabricated using default EOS M270 parameters. The sample is fabricated in the positive Z-direction, as shown in Figure 8.1(b). The hollow region is filled with IN625 powder particles due to the nature of the process, which remains contained inside the cylinder after fabrication.
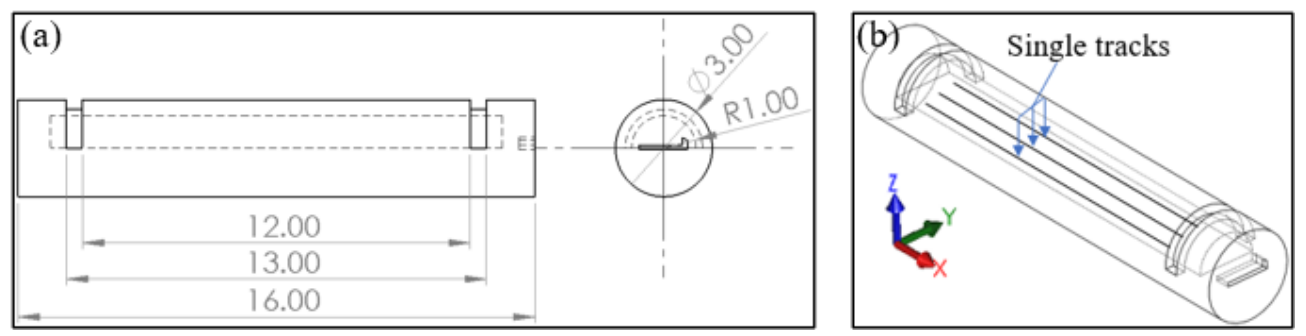

Figure 8.1 (a) CAD model of the specimen (unit in mm) and (b) Arrangement of the single tracks on top of the semi-cylinder base. 
Linear energy density (LED) is the ratio of laser power and scan speed,

$$
\operatorname{LED}\left(\frac{J}{m m}\right)=\frac{P(W)}{v\left(\frac{m m}{s}\right)}
$$

The laser power, scan speed, and the corresponding LEDs used in this study are listed in Table 8.1. In this study, the scan speeds from $200 \mathrm{~mm} / \mathrm{s}$ to $1200 \mathrm{~mm} / \mathrm{s}$ is used for a constant power of $195 \mathrm{~W}$, while the constant speed of $200 \mathrm{~mm} / \mathrm{s}$ is used for power ranging from $105 \mathrm{~W}$ to $195 \mathrm{~W}$. This design resulted in twelve LEDs ranging from 0.16 $\mathrm{J} / \mathrm{mm}$ to $0.98 \mathrm{~J} / \mathrm{mm}$. Besides obtaining the porosity at different LEDs, it is desired to observe the variation in the melt pool with the change in speed at constant power and change of power at a constant speed. Hence, metallography is performed to reveal the melt pool boundary.

Table 8.1 Process parameters used to investigate energy LED $(\mathrm{J} / \mathrm{mm})$ effect

\begin{tabular}{|l|l|l|l|l|l|l|}
\hline Constant & \multicolumn{5}{|c|}{ Variable parameter } \\
Parameter & \multicolumn{5}{|c|}{ (LED, J/mm) } \\
\hline $195 \mathrm{~W}$ & $\begin{array}{l}200 \mathrm{~mm} / \mathrm{s} \\
(0.98)\end{array}$ & $\begin{array}{l}400 \mathrm{~mm} / \mathrm{s} \\
(0.49)\end{array}$ & $600 \mathrm{~mm} / \mathrm{s}$ & $800 \mathrm{~mm} / \mathrm{s}$ & $1000 \mathrm{~mm} / \mathrm{s}$ & $1200 \mathrm{~mm} / \mathrm{s}$ \\
& & $(0.33)$ & $(0.24)$ & $(0.20)$ & $(0.16)$ \\
\hline $200 \mathrm{~mm} / \mathrm{s}$ & $105 \mathrm{~W}$ & $120 \mathrm{~W}$ & $135 \mathrm{~W}$ & $150 \mathrm{~W}$ & $165 \mathrm{~W}$ & $180 \mathrm{~W}$ \\
& $(0.53)$ & $(0.6)$ & $(0.68)$ & $(0.75)$ & $(0.83)$ & $(0.9)$ \\
& & & & & & \\
\hline
\end{tabular}

Ti-6Al-4V single track experiment performed with the SLM process showed that the power has more effect on porosity than the scan speed [106]. However, the relationship between the parameters and the melt pool boundary was not established. In 
this study, an experiment is designed for IN625 to study the change in the melt pool depth and porosity with different levels of power and speed from the same LED. The LEDs and the respective laser power and scan speeds are listed in Table 8.2 and Table 8.3. The LED from conduction mode $(0.48 \mathrm{~J} / \mathrm{mm})$ and keyhole mode $(0.75 \mathrm{~J} / \mathrm{mm})$ are selected to investigate the effect of the power and speed on the porosity. Besides, metallography is performed to reveal the melt pool boundary of IN625 single tracks formed on top of the SLM fabricated base [62]. The transverse and longitudinal melt pool boundary is obtained, and the effect of power and speed within the same LED on the melt profile characteristics is investigated.

Table 8.2 Process parameters selected from the constant energy density of $0.48 \mathrm{~J} / \mathrm{mm}$.

\begin{tabular}{|l|l|l|l|l|l|l|l|l|}
\hline Speed(mm/s) & 50 & 100 & 150 & 200 & 250 & 300 & 350 & 406 \\
\hline Power (W) & 24 & 48 & 72 & 96 & 120 & 144 & 168 & 195 \\
\hline
\end{tabular}

Table 8.3 Process parameters selected from the constant energy density of $0.75 \mathrm{~J} / \mathrm{mm}$.

\begin{tabular}{|l|l|l|l|l|l|l|l|l|l|}
\hline Speed(mm/s) & 50 & 75 & 100 & 125 & 150 & 175 & 200 & 225 & 250 \\
\hline Power (W) & 38 & 56 & 75 & 94 & 113 & 131 & 150 & 169 & 188 \\
\hline
\end{tabular}

Figure 8.2(a) shows the cylinder samples attached to the build platform through the block support. The cylinder samples were separated from the build plate, and the excess support attached to the sample surface was removed by polishing. Bruker SkyScan 1173 micro-CT scanner, which is shown in Figure 8.2(b), was then used to scan the 
cylinder samples with $6 \mu \mathrm{m}$ pixel size at 2000 magnification. The rotation step of $0.2^{\circ}$ was used, and $360^{\circ}$ scanning was performed to obtain 1800 raw images. The raw images were reconstructed using standard reconstruction procedures discussed in the literature [106] and finally analyzed. After the CT scanning is completed, the samples were sectioned, polished, and etched to reveal the transverse melt profile. The optical microscope is used to observe the melt pool boundary, and the width and depth of the single tracks are measured. Besides, the shape of the melt pool boundary is used to separate between the conduction mode and keyhole mode melting.

(a)

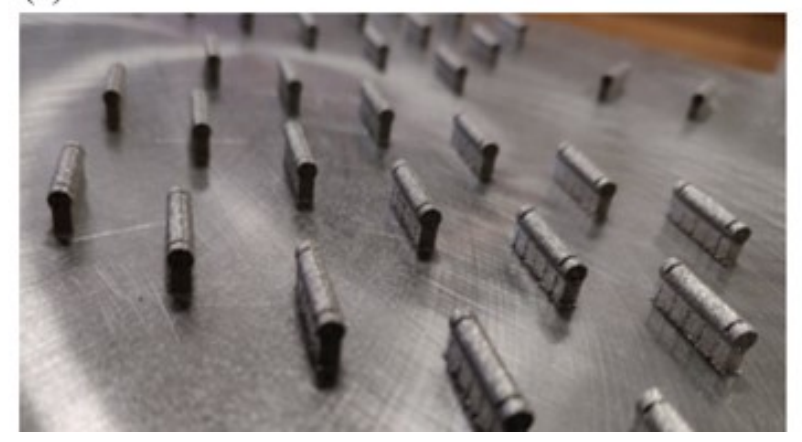

(b)

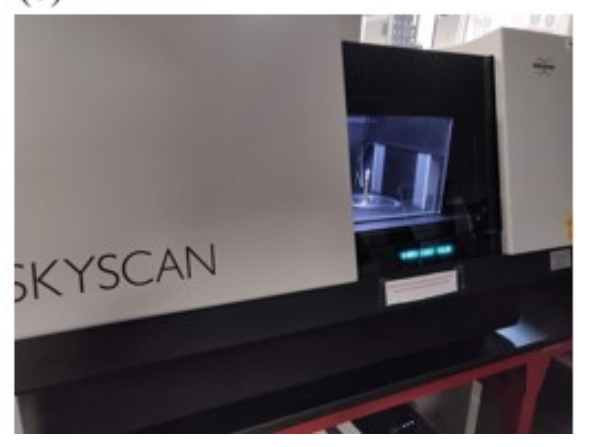

Figure 8.2 (a) Cylinder samples fabricated on the build plate, and (b) Bruker 1173 microCT scanner setup.

\subsection{Numerical Approach}

A powder scale numerical model is developed using FLOW-3D commercial software. An opensource discrete element method (DEM) package is used to simulate the spreading of the IN625 powder layer [107], and the powder layer is imported to the FLOW-3D software to perform the thermo-fluid simulation. Figure 8.3 shows the simulation domain used in this study. The dimension of the solid substrate is $500 \mu \mathrm{m} \times$ $300 \mu \mathrm{m} \times 250 \mu \mathrm{m}$, and a layer of powder is defined on top of the solid substrate. The 
short scan length simulation is performed to analyze the variation in temperature distribution and laser penetration at a different level of power and scan speed. The scan length of $250 \mu \mathrm{m}$ is used, which is sufficient to capture the change in the temperature and the formation of the keyhole [107].

IN625 material properties are assigned to both powder and solid substrate. The temperature dependent IN625 material properties used in the simulation are shown in Figure 8.4. Besides, other properties are listed in Table 8.4. The laser source is modeled as a gaussian heat source, and complex physics such as laser multiple reflections, material evaporation, and recoil pressure are included in the model to accurately predict the laser melting and keyhole formation.

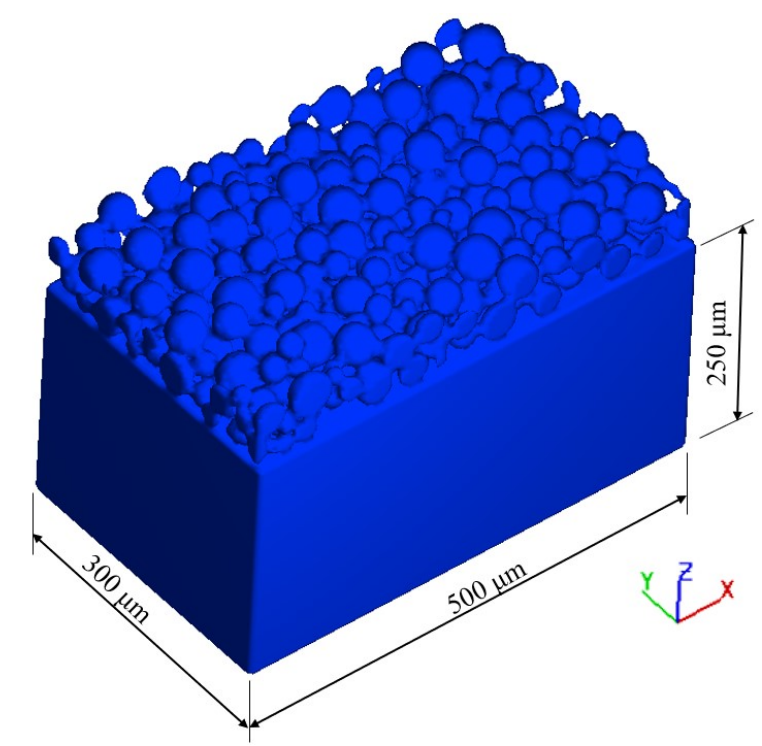

Figure 8.3 Simulation domain used to perform a $250 \mu \mathrm{m}$ long single-track simulation. 

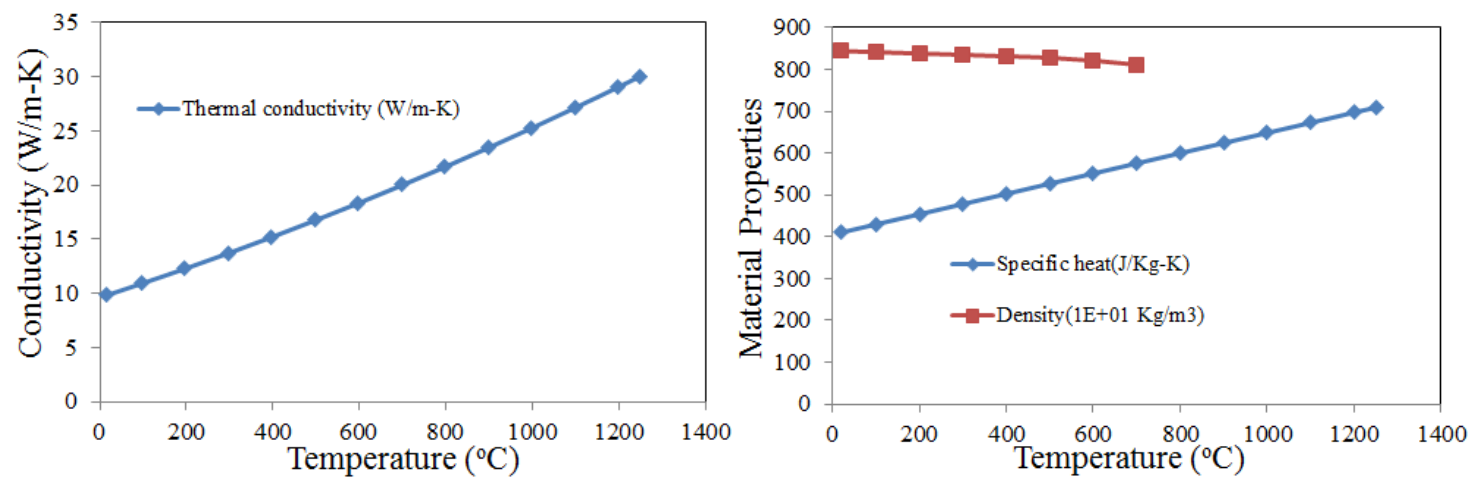

Figure 8.4 Temperature dependent material properties of Inconel 625[25].

Table 8.4 Properties of In625 in a thermo-fluid simulation.

\begin{tabular}{ll}
\hline Parameters & Values \\
\hline Solidus temperature, $T_{S}(\mathrm{~K})$ & 1563 \\
Liquidus temperature, $T_{L}(\mathrm{~K})$ & 1623 \\
Boiling temperature, $\mathrm{T}_{\mathrm{v}}(\mathrm{K})$ & 3188 \\
Viscosity $(\mathrm{kg} / \mathrm{m}-\mathrm{s})$ & 0.007 \\
Surface tension $(\mathrm{N} / \mathrm{m})$ & 1.8 \\
Surface tension gradient $(\mathrm{N} / \mathrm{m}-\mathrm{K})$ & $-2 \mathrm{E}-05$ \\
\hline
\end{tabular}

\subsection{Results and Discussion}

\subsubsection{Line energy density effect}

The CT scanned cylinder samples are analyzed to obtain the pore number and pore volume formed inside the single tracks. Figure $8.5(\mathrm{a})$ shows the $3 \mathrm{D}$ image of a typical cylinder sample obtained after the $\mathrm{CT}$ image reconstruction. The grayscale image is based on the density of the sample. The light gray region is the solid In625, dark gray contained inside the cylinder is the In625 powder, and the black region represents the void. A transverse section of the sample is shown in Figure 8.5(b). Three tracks are identified based on the surface profile, and one of the tracks shows the formation of the pore inside the track. Besides, the density-based transfer function is modified to remove 
the powder material inside, and the 3D clipping is performed to observe the single track formed over the base, as shown in Figure 8.5(c). This feature enables the comparison of the surface morphologies formed with different laser parameters.
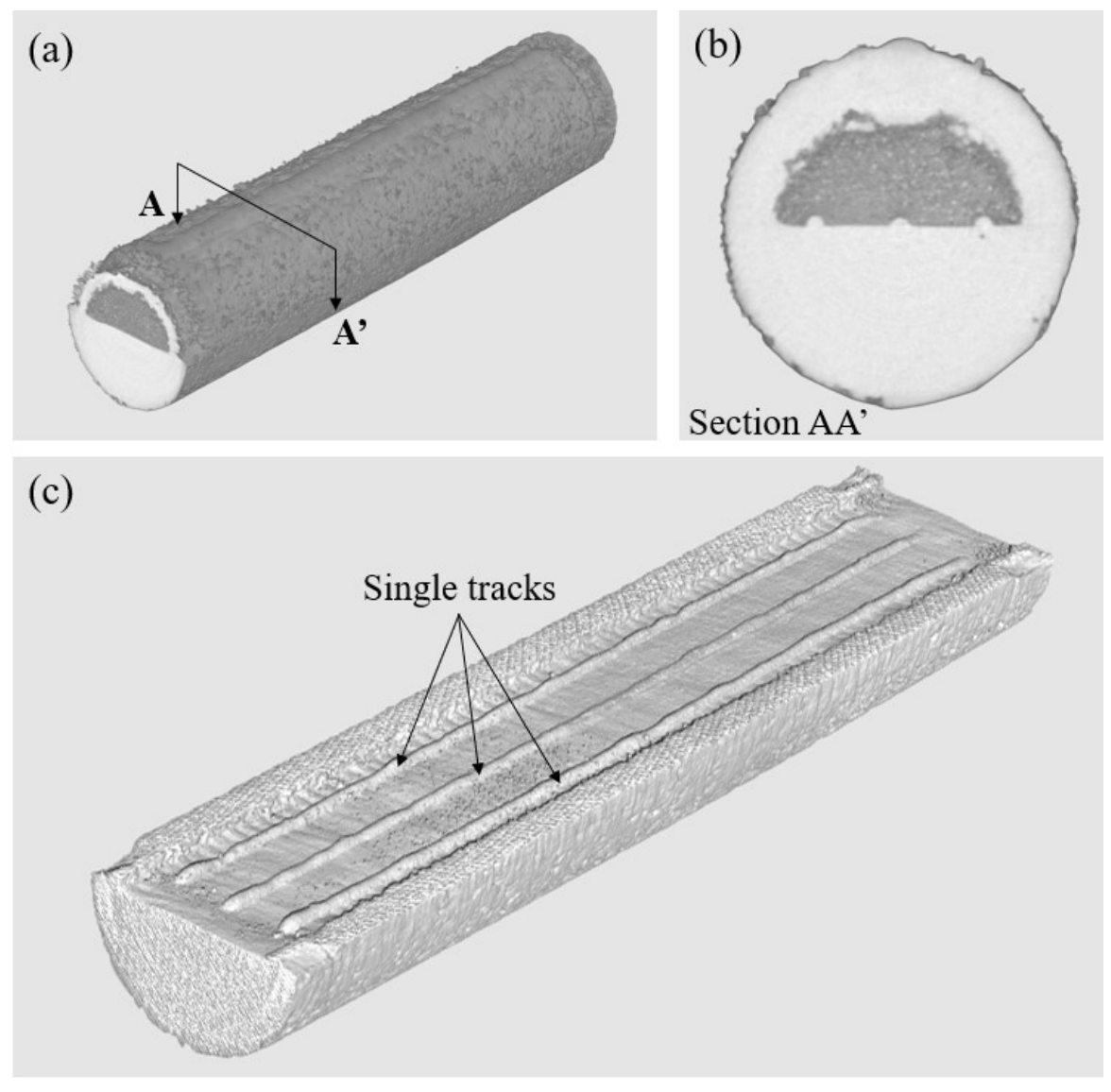

Figure 8.5 (a) CT scanned cylinder after reconstruction, (b) Transverse cross section, and (c) Modification of the transfer function to remove the powder particles and observe the single tracks.

Figure 8.6 presents the micro-CT image of the keyhole pores formed with 0.98 $\mathrm{J} / \mathrm{mm}(195 \mathrm{~W}$ and $200 \mathrm{~mm} / \mathrm{s})$ LED along the scan direction. The pores are randomly distributed, with a huge variation in the size. Besides, due to the deep penetration melting and nature of the pore formation, the pores are formed deep inside the track. However, the depth of the single track is not known from micro-CT, although the formation of the 
pore is apparent. Figure 8.6 also shows the 3D rendered pores, which show that the pores formed are mostly spherical.

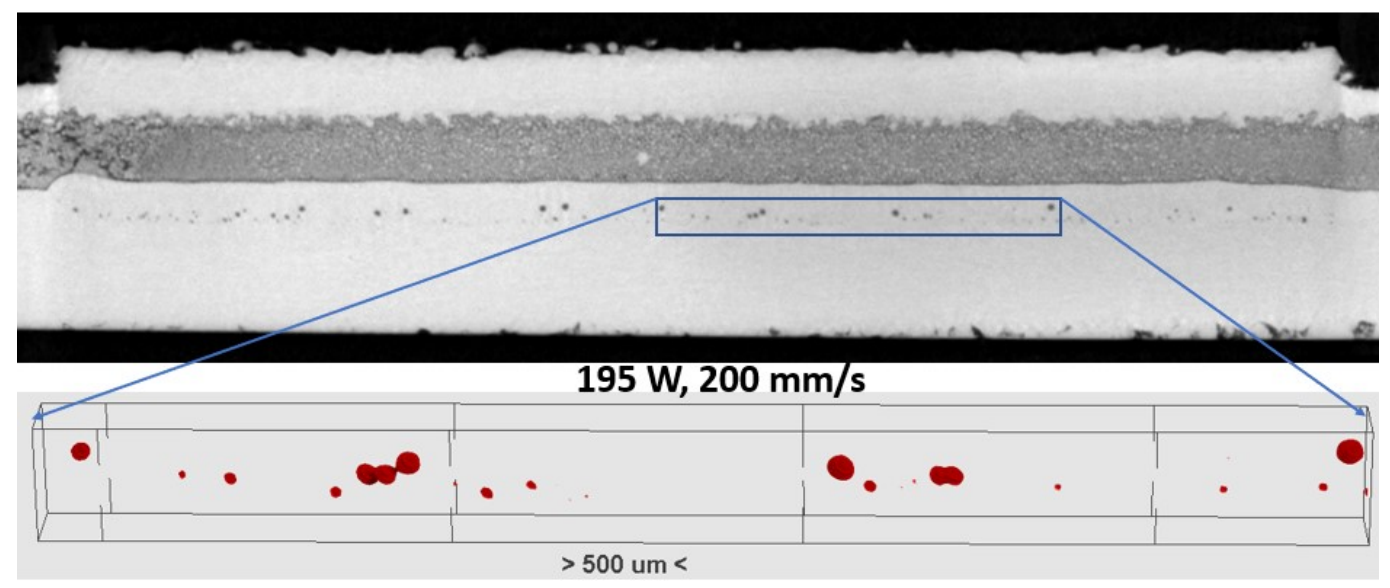

Figure 8.6 Pore formed with $0.98 \mathrm{~J} / \mathrm{mm}(195 \mathrm{~W}$ and $200 \mathrm{~mm} / \mathrm{s})$.

The pores formed inside the single tracks are measured, and Figure 8.7 presents the average pore number and volume from three replicates for all the investigated LEDs. The error bar indicates the standard deviation from three replicates of the single tracks. In general, the single-track porosity increased with the increase in LED, and the maximum porosity is observed at the LED of $0.90 \mathrm{~J} / \mathrm{mm}(180 \mathrm{~W}$ and $200 \mathrm{~mm} / \mathrm{s})$. The increase in porosity is related to the formation of the keyhole due to the increase in energy density. However, the highest LED of $0.98 \mathrm{~J} / \mathrm{mm}(195 \mathrm{~W}$ and $200 \mathrm{~mm} / \mathrm{s})$ resulted in a lower average pore number and volume compared to $0.9 \mathrm{~J} / \mathrm{mm}$.

It is also noted that the number and volume of the pores formed with the same LEDs are smaller for IN625 with $40 \mu \mathrm{m}$ layer thickness compared to the Ti-6Al-4V with $30 \mu \mathrm{m}$ layer thickness [106]. The data analytical study showed that the small variation in the layer thickness has minimal effect on the melt pool depth [9]. Hence, such a 
difference in the pore number and pore volume may be due to the difference in the thermal properties between the two materials. This difference in the melt pool characteristics between different materials shows the importance of including the material properties while establishing the relationship between energy density and melting modes.

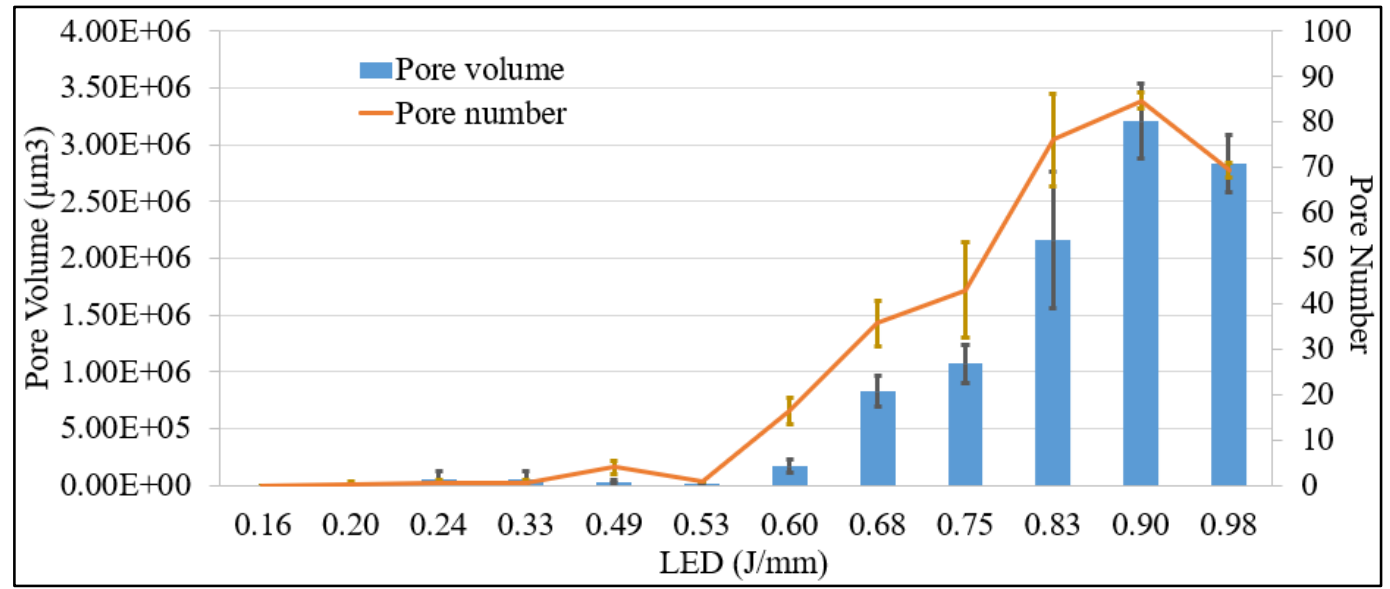

Figure 8.7 Pore volume and pore number from different LEDs.

The average diameter and depth of the pores are also a function of LED. The average pore depth and the standard deviation of the pores formed at different LEDs are shown in Figure 8.8. The average depth of the pores increased with the increase in LED, which may be due to the increase in keyhole depth formed with increasing LEDs. Besides, the average pore size also increased with an increase in LED. The increase in the size may be related to the size of the vapor opening, which is bigger at higher LED [107]. 


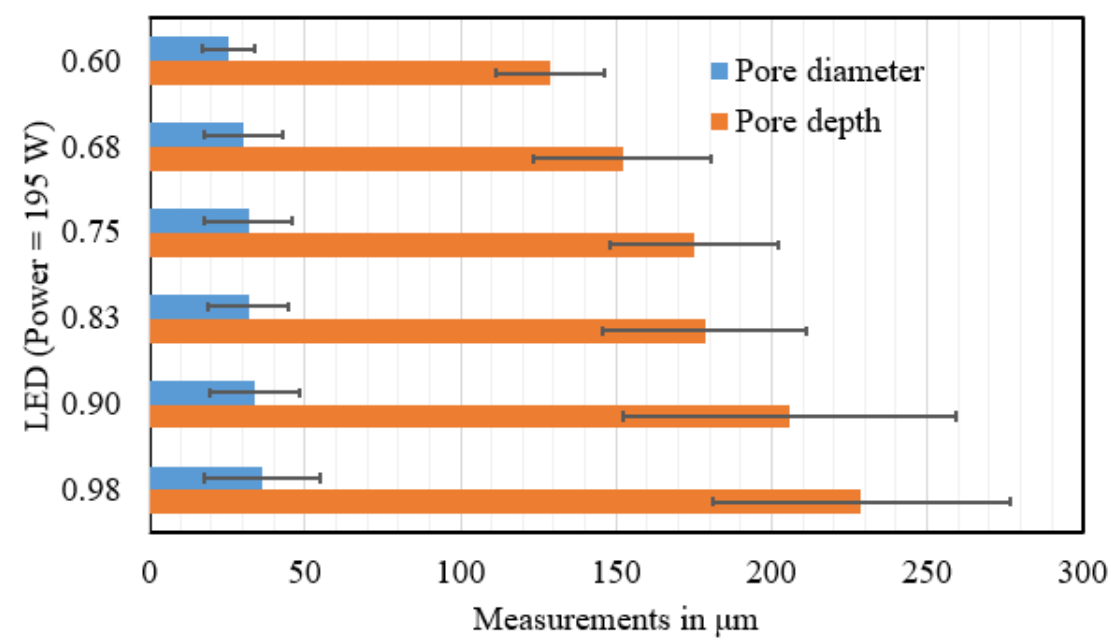

Figure 8.8 Average pore diameter and depth

The pore depth measurements suggested that deeper melt pools are formed with higher LEDs. Hence, the single-track samples are cut in the middle of the track to obtain the transverse melt pool boundaries and differentiate different melting modes. The samples are polished and then etched with modified Kalling's reagent (40 $\mathrm{ml}$ distilled water $+480 \mathrm{ml} \mathrm{HCl}+48 \mathrm{mg} \mathrm{Cucl} 2)$ to reveal the melt pool boundary. Figure 8.9 shows the transverse melt pool boundary formed with different LEDs. The depth of the melt pool increased with the increase in LED. For the same power, the width and depth of the melt pool decreased with an increase in speed due to the decrease in the interaction time. However, for the same speed, the depth increased with an increase in power due to the increase in the laser penetration. 


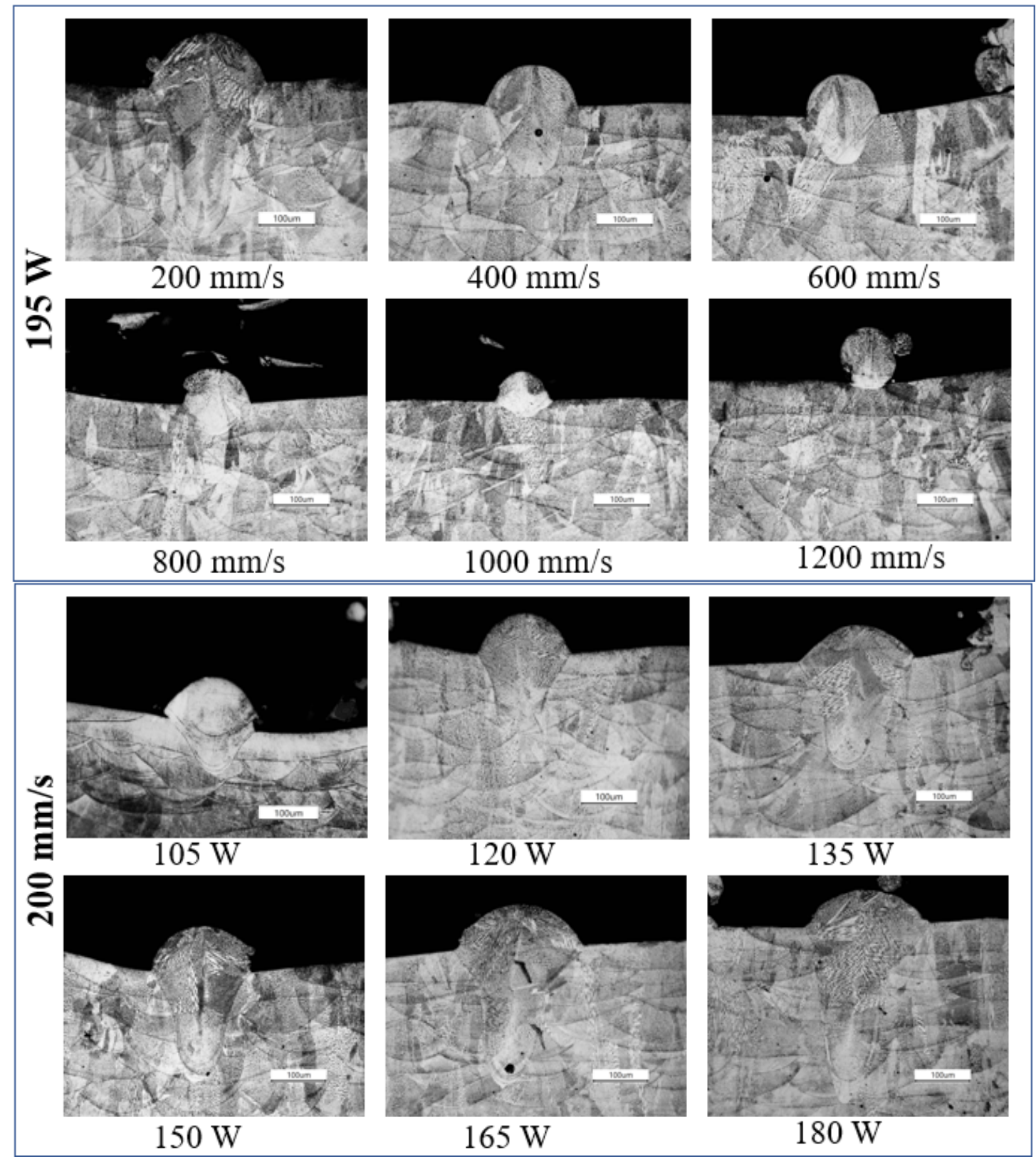

Figure 8.9 Transverse melt profile from different LEDs.

The width and the depth of the single tracks are measured from the transverse profile. Figure 8.10 shows the measurement approach of the track width and depth and the relationship between LED and track width and depth. The track width and depth increased with increasing LED. However, the increase in depth is significant compared to 
the width. The width of the track is higher than the depth for all the conduction mode melting, while the depth is higher than the width for the deep keyhole mode melting. A second-order polynomial curve is fitted to both depth and width, which shows that the depth is increasing almost linearly with LED. However, the width of the track increased with an increase in LED within conduction mode but did not show an obvious increase within the keyhole regime. The maximum width of the track may be limited by the beam diameter and increasing the energy density with the same beam diameter may increase the depth rather than width after reaching a certain limit. There are some cases where the higher LED resulted in the shallower melt pool. The LED of $0.53 \mathrm{~J} / \mathrm{mm}(105 \mathrm{~W}$ and 200 $\mathrm{mm} / \mathrm{s}$ ) resulted in the depth lower than $0.49 \mathrm{~J} / \mathrm{mm}(195 \mathrm{~W}$ and $400 \mathrm{~mm} / \mathrm{s})$. This behavior may be due to the significant difference in the power between the two LEDs. However, the LED of $0.98 \mathrm{~J} / \mathrm{mm}$ (195 and $200 \mathrm{~mm} / \mathrm{s}$ ) also resulted in shallower melt pool compared to the LED of $0.90 \mathrm{~J} / \mathrm{mm}(180 \mathrm{~W}$ and $200 \mathrm{~mm} / \mathrm{s})$, although $0.98 \mathrm{~J} / \mathrm{mm}$ had a higher power. Such anomaly may be due to the variation in the melt pool depth during the keyhole melting [36]. The longitudinal metallography should be performed to obtain the variation in depth along the laser travel direction.

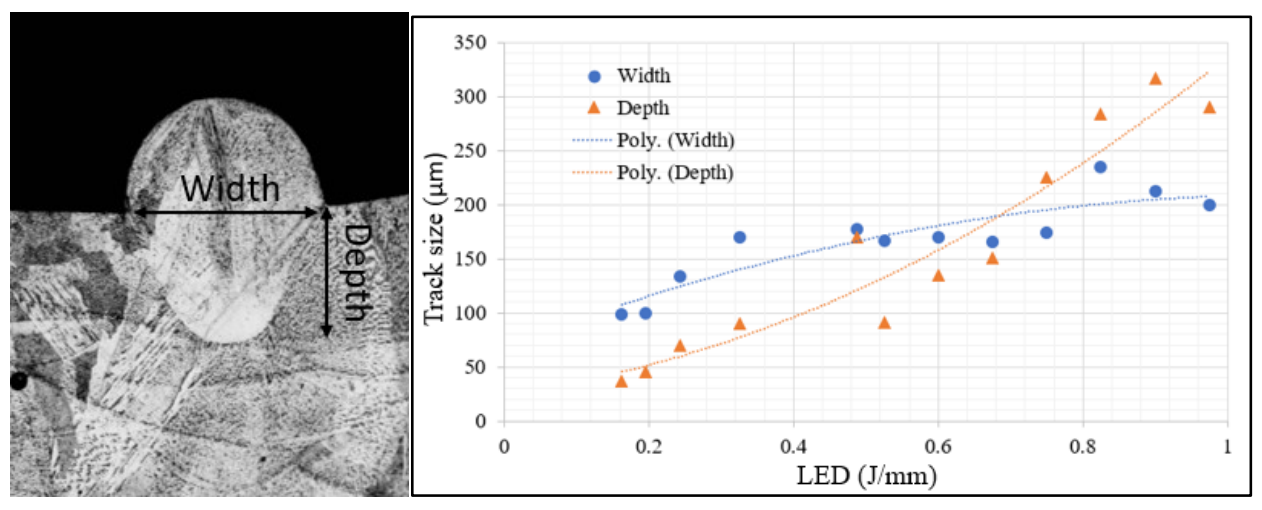

Figure 8.10 Track width and depth measured from the transverse profile of different LEDs. 


\subsubsection{Effect of power and speed at the same energy density}

The Ti-6Al-4V experiment showed that the pore number and pore volume increased with the increase in power and speed at the same energy density to a certain level and then dropped with a further increase in power and speed [19]. In this study 0.48 $\mathrm{J} / \mathrm{mm}$ and $0.75 \mathrm{~J} / \mathrm{mm}$ LED are selected, and the average pore number and pore volume from three replicates are shown in Figure 8.11. The LED of $0.48 \mathrm{~J} / \mathrm{mm}$ resulted in fewer pores compared to the $0.75 \mathrm{~J} / \mathrm{mm}$. Besides, the porosity increased with an increase in power for both LEDs. However, the porosity was not maximum for the highest power. The numerical study showed that the formation of the keyhole pores is due to instabilities in the keyhole wall because of the interaction between surface tension pressure and recoil pressure [17]. Within the keyhole mode at the same energy density, the increase in power would increase the radius of the vapor opening. At a certain point, the instability between the surface tension and vapor opening leads to the formation of many the pores due to the collapse of the keyhole wall. However, with further increase in the power, the keyhole opening is large enough so that the surface tension pressure is unable to close the keyhole wall, leading to a stable keyhole. This reduces the chance of the pore formation. Besides, the mode of melting changed from incomplete to conduction and finally to keyhole with increasing power and speed, while keeping the LED as constant. 

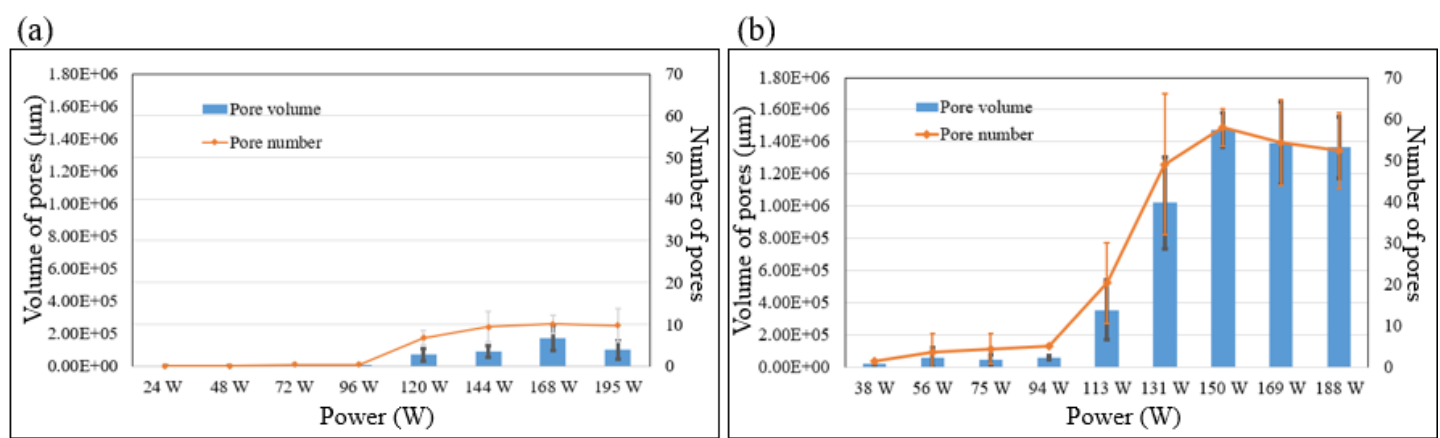

Figure 8.11 Pore volume and pore number from different power and speed when (a) LED $=0.48 \mathrm{~J} / \mathrm{mm}$ and (b) LED $=0.75 \mathrm{~J} / \mathrm{mm}$.

The surface morphology and the respective transverse melt profile of the single tracks formed with different levels of power and scan speed from LED of $0.48 \mathrm{~J} / \mathrm{mm}$ are shown in Figure 8.12. At low power and low speed ( $24 \mathrm{~W}$ and $50 \mathrm{~mm} / \mathrm{s})$, the discontinuous track is formed. The discontinuity corresponds to the insufficient melting, which is usually the case with very low LEDs. In this case, even if the LED is high, the power is very low. Hence, the low power resulted in the lower melt pool temperature, and consequently, the laser penetration was insufficient to form a continuous single track. The increase in power and speed led to the formation of continuous tracks. The melt pool depth slowly increased within the conduction modes from $48 \mathrm{~W}$ and $100 \mathrm{~mm} / \mathrm{s}$ to $96 \mathrm{~W}$ and $200 \mathrm{~mm} / \mathrm{s}$. However, there is a sudden increase in the depth when the laser power increased to $120 \mathrm{~W}$, suggesting the keyhole mode melting. Figure 8.12 shows a clear transition from the incomplete melting to the conduction melting and finally to the keyhole melting with an increase in the laser power and scan speed at the same energy density. Although the depth of the melt pool increased with increasing power and speed, the average volume and number of pores were not maximum for the maximum speed and power used. This shows that the porosity is not directly related to the depth of the 
keyhole, rather its occurrence depends on the stability of the keyhole formed, during the melting process. Besides the depth of the melt pool, the width of the track also increased with an increase in the power and speed from incomplete melting to the keyhole melting. However, the track width did not show an obvious increase with the increase in power within the keyhole mode, which is consistent with the result obtained in the LED effect study discussed in the previous section.

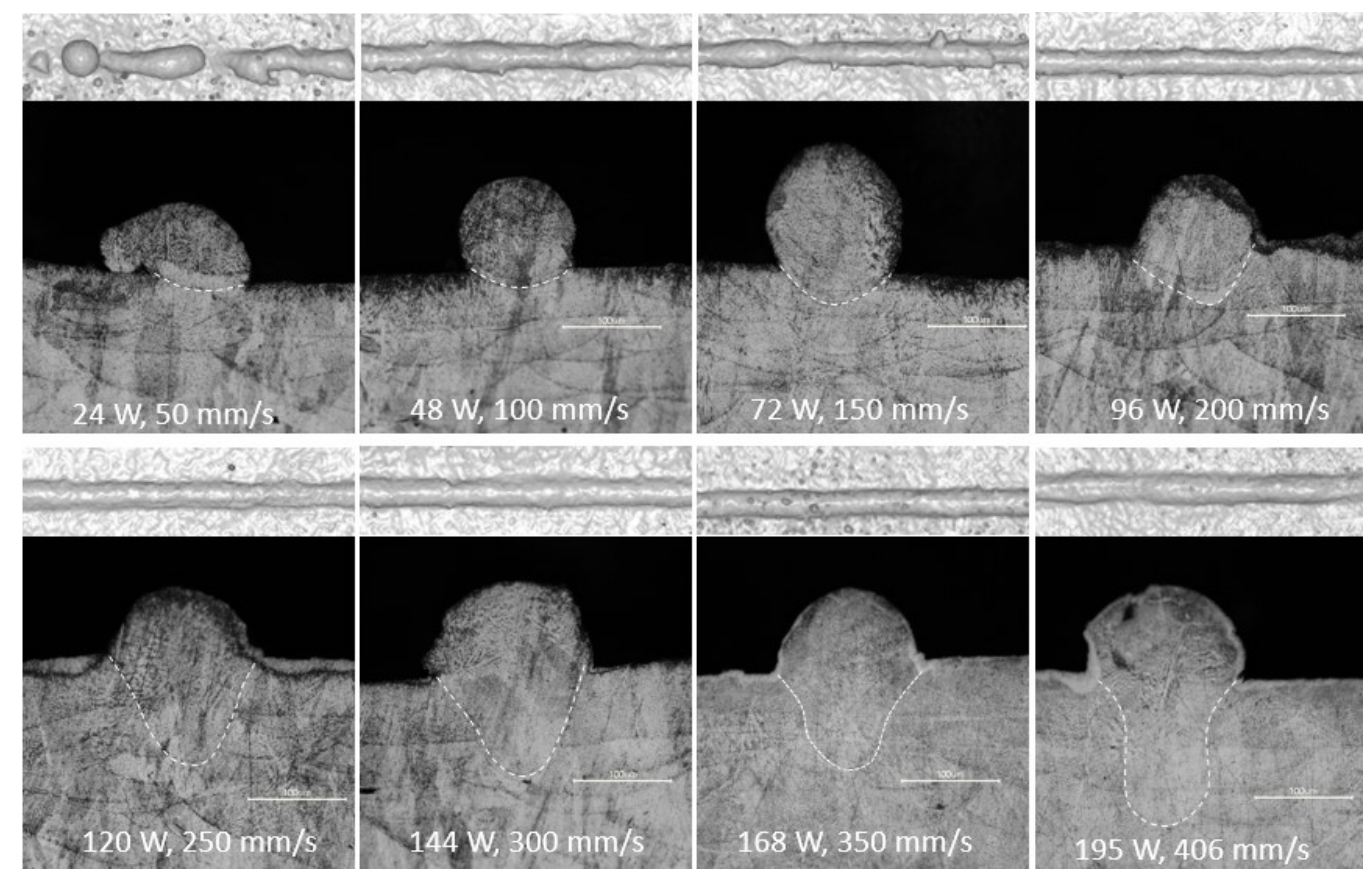

Figure 8.12 Transverse melt profile obtained from $\mathrm{LED}=0.48 \mathrm{~J} / \mathrm{mm}$ single tracks.

Figure 8.12 clearly shows that the melt pool characteristic is not the function of the linear energy density. As the melt pool depth depends on the power, speed, and the material properties, the normalized enthalpy is used for better representation of the data [89]. The calibrated normalized enthalpy shown in equation 1 is used [36],

$$
\frac{\Delta H}{h_{s}}=\frac{A P}{\pi h_{s} \sqrt{D u \sigma^{3}}}
$$


where, $\Delta \mathrm{H}$ is the specific enthalpy, $\mathrm{h}_{\mathrm{s}}$ is the enthalpy at melting, $\mathrm{A}$ is the absorptivity, $\mathrm{P}$ is the laser power, $\mathrm{D}$ is the thermal diffusivity, $\mathrm{u}$ is the laser speed and $\sigma$ is the Gaussian beam size. The melt pool depth is the function of normalized enthalpy; therefore, normalized depth may be written as,

$$
\text { Normalized depth }=f n\left(\frac{A P}{\pi h_{s} \sqrt{D u \sigma^{3}}}\right) \text {. }
$$

The dimensionless depth normalized to the Gaussian beam size is plotted against the normalized enthalpy as shown in Figure 8.13. As the depth to half-width ratio is used to distinguish the keyhole melting and the conduction melting, it is also included in Figure 8.13. Generally, the normalized depth and the depth to half-width ratio increased with increasing enthalpy. However, there are certain cases where higher enthalpy resulted in lower depths.

The transverse depth is used to assess the effect of enthalpy on the depth. However, it is important to note that the depth would change along the longitudinal direction and the melt pool profile taken at a single transverse direction may sometimes lead to dubious comparisons. Hence, a longitudinal melt profile is obtained through metallography. Figure 8.14 presents the two examples of the longitudinal melt pool boundaries obtained with $0.48 \mathrm{~J} / \mathrm{mm}(120 \mathrm{~W}, 250 \mathrm{~mm} / \mathrm{s}$ and $144 \mathrm{~W}, 300 \mathrm{~mm} / \mathrm{s})$, and two with $0.75 \mathrm{~J} / \mathrm{mm}(131 \mathrm{~W}, 175 \mathrm{~mm} / \mathrm{s}$ and $150 \mathrm{~W}, 200 \mathrm{~mm} / \mathrm{s})$. The variation in the depth along the scan direction is evident from the longitudinal melt pool boundary. Moreover, the depth of the melt pool for $144 \mathrm{~W}$ is higher than that of $120 \mathrm{~W}$. The depth measured from the transverse profile is $108 \mu \mathrm{m}$, and the range of the depth measured from the longitudinal profile is $88 \mu \mathrm{m}$ to $175 \mu \mathrm{m}$. The depth of the transverse melt pool would, 
therefore, change according to the location. The range of the depths obtained for different cases is included as an error bar in Figure 8.13. The longitudinal profiles of $0.75 \mathrm{~J} / \mathrm{mm}$ show that the keyhole pores are usually formed at the bottom of the melt pool.

Based on the depth to width ratio, the conduction mode melting occurred up to 96 $\mathrm{W}$ and $200 \mathrm{~mm} / \mathrm{s}$, which corresponded to the normalized enthalpy of 5.5. As the power and speed increased to $120 \mathrm{~W}$ and $250 \mathrm{~mm} / \mathrm{s}$, the depth to half-width ratio is greater than 1 , suggesting a formation of a keyhole, and the corresponding enthalpy is around 6 . In this case, the transition from the conduction to the keyhole mode melting is observed to be around $\Delta \mathrm{H} / \mathrm{h}_{\mathrm{s}}=6$. Two lines are drawn to distinguish the keyhole modes based on the normalized depth and the depth to width ratios. The horizontal line is drawn for a depth ratio of 1 , which would separate the keyhole parameters based on the depth to half-width ratio. The vertical line separates the keyhole parameters based on the normalized enthalpy. The use of enthalpy as a design parameter is, therefore, a simple approach to identify the process parameters as the keyhole or the conduction parameters. The frequency of the pore formation is lower for $131 \mathrm{~W}$, hence resulted in the fewer pore number compared to the $150 \mathrm{~W}$ power.
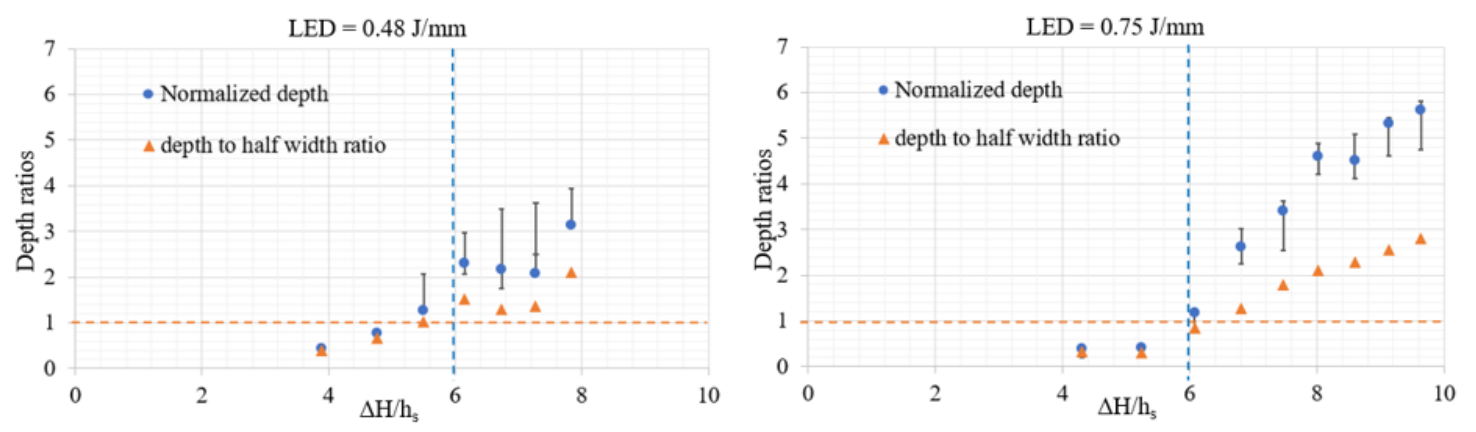

Figure 8.13 The effect of normalized enthalpy on the non-dimensional depth and depth to width ratio. 

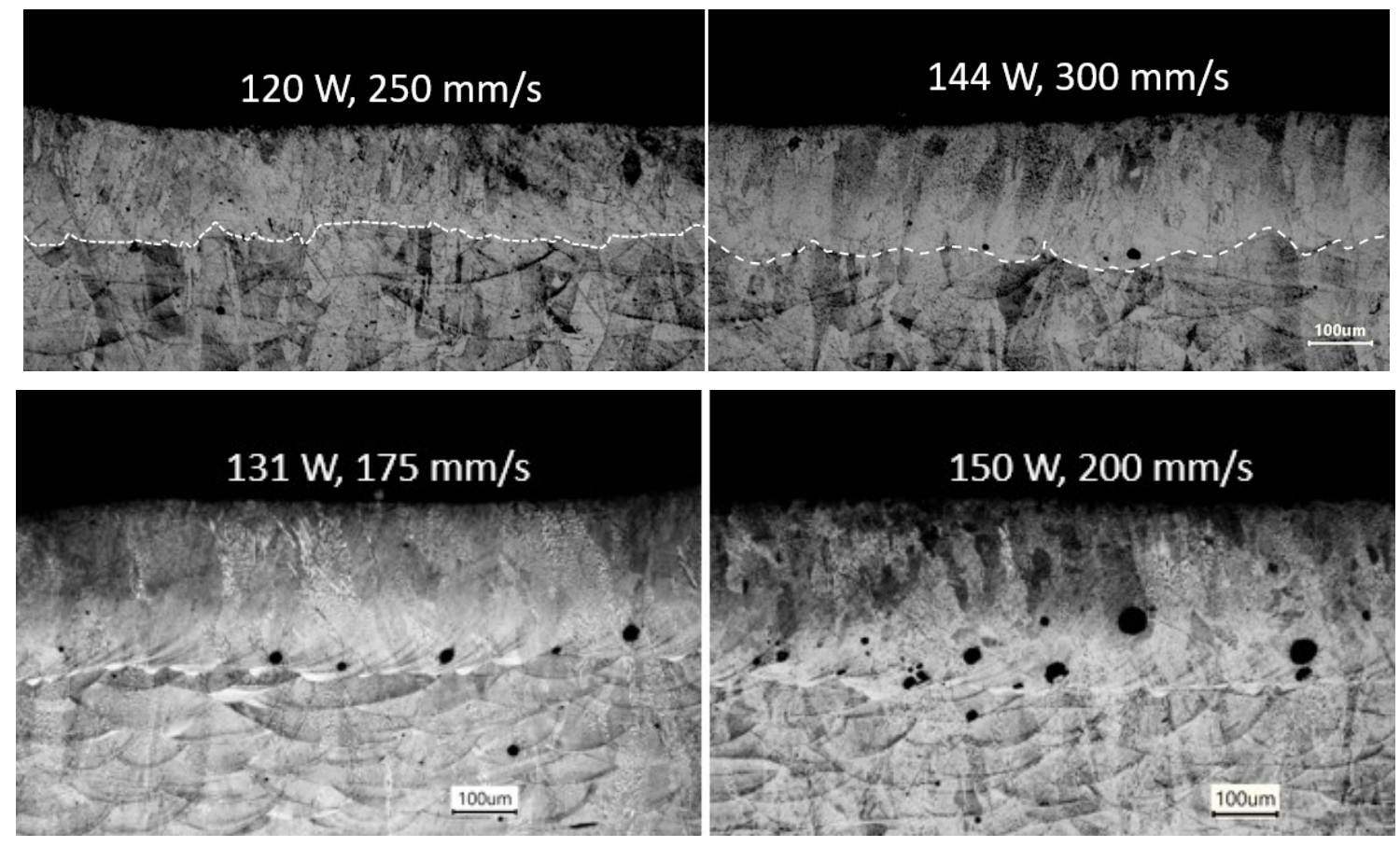

Figure 8.14 Longitudinal melt profile obtained from $\mathrm{LED}=0.48 \mathrm{~J} / \mathrm{mm}$ and $0.75 \mathrm{~J} / \mathrm{mm}$ single tracks.

As the power has more effect on the depth than the speed, it is expected to have differences in the depth with the change in the level of power and speed, even if the LED is kept constant. Besides, laser power has more effect on the peak temperature of the melt pool during the SLM process, and the temperature increases with an increase in power [106]. Numerical models may be used to predict the real-time temperature and flow of the melt pool, which determines the shape of the melt pool boundary. Hence, a microscale numerical model is developed to predict the melt pool formation with different levels of power and speed.

Figure 8.15 shows the temperature distribution along the longitudinal direction when the laser has traveled $100 \mu \mathrm{m}, 150 \mu \mathrm{m}, 200 \mu \mathrm{m}$, and $250 \mu \mathrm{m}$. At low power and speed (48 W and $100 \mathrm{~mm} / \mathrm{s}$ and $72 \mathrm{~W}$ and $150 \mathrm{~mm} / \mathrm{s}$ ), the melting occurs purely by 
conduction. The heat absorbed by the free surface exposed to the laser is diffused to the substrate, which melts the material beneath, forming a shallow melt pool. The temperature profile shows that some region exceeds the evaporation temperature, however, the material evaporation is not enough to generate the recoil pressure to form a depression. The melt pool formed by the pure conduction is very shallow, with the depth to width ratio being well below 1 . For the laser power of $92 \mathrm{~W}$, a depression is formed, leading to a deeper melt pool. However, it is not regarded as the keyhole mode melting in this study as the depth to half-width ratio about 1 . The vapor opening has a larger diameter and remains open all the time, hence the possibility of the pore formation due to the collapse of the vapor opening is minimal. When the power is increased to $120 \mathrm{~W}$, the region with a temperature greater than the evaporation temperature is very high, and the material evaporation exerts the recoil pressure forming a keyhole. Hence, the shape of the transverse profile is determined by the shape of the keyhole. As the temperature exceeds the evaporation temperature, there is a significant increase in the recoil pressure, due to the exponential relationship [86]:

$$
P_{\text {recoil }}=0.54 P_{0} \exp \left(\frac{\Delta H_{v}\left(T-T_{v}\right)}{R T T_{v}}\right)
$$

where $P_{0}$ is the atmospheric pressure, $\Delta H_{v}$ is the specific enthalpy of metal vapor, $T_{v}$ is the boiling temperature, $\mathrm{T}$ is the surface temperature and $\mathrm{R}$ is the universal gas constant.

The vapor opening is smaller in $144 \mathrm{~W}$ and $300 \mathrm{~mm} / \mathrm{s}$ compared to $120 \mathrm{~W}$ and $250 \mathrm{~m} / \mathrm{s}$. This may lead to the instabilities within the keyhole, inducing the pore formation. The simulation predicted depths are close to the experimental depths. Figure 8.16 compares the depth to the half-width ratio predicted by the simulation with the 
experiment. The simulation depth to the half-width ratio increased linearly with the increase in the normalized enthalpy. The study shows that the small-scale simulation may be used to predict different modes of melting, which is also able to hint at the possible instabilities in the keyhole.

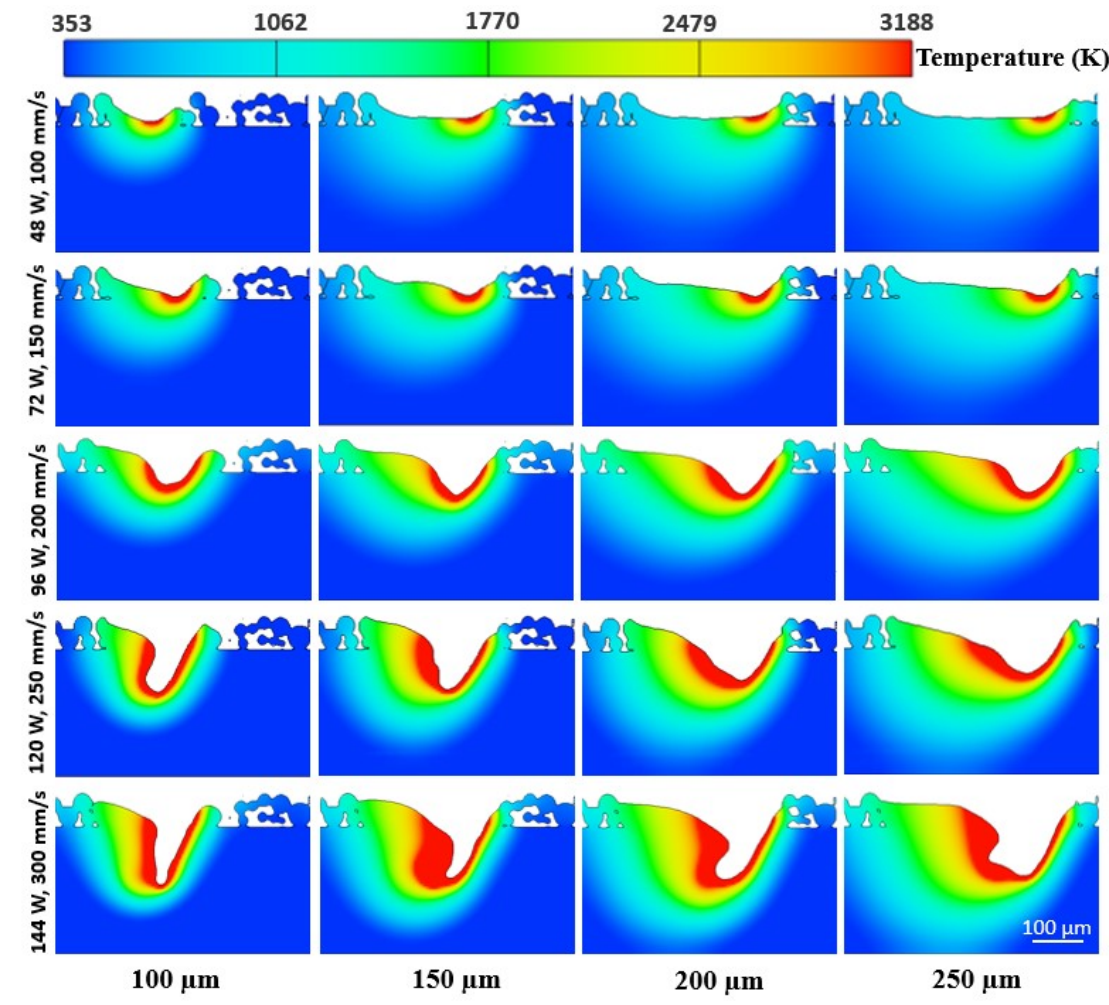

Figure 8.15 Temperature profile and the melt pool shape when laser has travelled 100 $\mu \mathrm{m}, 150 \mu \mathrm{m}, 200 \mu \mathrm{m}$ and $250 \mu \mathrm{m}(\mathrm{LED}=0.48 \mathrm{~J} / \mathrm{mm})$.

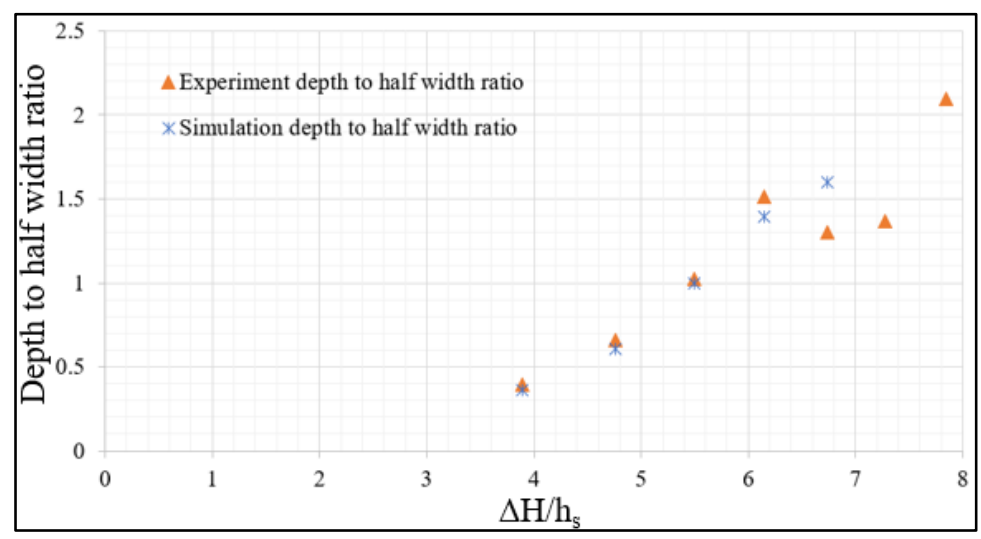

Figure 8.16 Figure 16. Depth to half width ratio comparison between simulation and experiment with LED $=0.48 \mathrm{~J} / \mathrm{mm}$. 


\subsection{Conclusions}

The melting modes in the SLM process depend on the process parameters. Hence, the effect of LED on the melting modes and keyhole porosity is investigated in this study. EOS M270 is used to fabricate Inconel 625 single tracks with different levels of LEDs, ranging from $0.16 \mathrm{~J} / \mathrm{mm}$ to $0.98 \mathrm{~J} / \mathrm{mm}$. The pores formed inside the single tracks were measured with a micro-CT scanner, and metallography was conducted to obtain the transverse melt profile. Besides, the effect of different levels of power and speed under the two LEDs, $0.48 \mathrm{~J} / \mathrm{mm}$, and $0.75 \mathrm{~J} / \mathrm{mm}$, is investigated. The results and analyses lead to the following conclusions:

- $\quad$ The mode of melting progressed from conduction to keyhole with increasing LED. The depth of the keyhole and the keyhole porosity generally increased with increasing LED. However, the maximum porosity was obtained at $0.90 \mathrm{~J} / \mathrm{mm}(180 \mathrm{~W}$, $200 \mathrm{~mm} / \mathrm{s})$ instead of the highest LED investigated, i.e., $0.98 \mathrm{~J} / \mathrm{mm}(195 \mathrm{~W}, 200 \mathrm{~mm} / \mathrm{s})$.

- The mode of melting progressed from incomplete melting to conduction melting, and finally to keyhole melting with an increase in the laser power and scan speed at the same LED. Besides, the depth of the melt pool increased consistently with the increase in laser power. However, the keyhole porosity did not increase with an increase in the melt pool depth, instead, the keyhole porosity increased to a certain level and then decreased with further increase in laser power.

- The numerical results show that the increase in the melt pool depth is due to the increase in the melt pool temperature and the vapor pressure. Besides, there is a significant increase in penetration with a small increase in laser power due to the 
exponential relationship between the recoil pressure and the surface temperature. The vapor opening in the conduction mode is stable, and the instability in the keyhole becomes evident with an increase in the depth of the keyhole.

- $\quad$ LED may not be used as a sole parameter to distinguish the mode of melting as the laser power has a greater effect on melt pool depth than the scan speed. Normalized enthalpy, which includes process parameters as well as material properties, may be more appropriate to explain the shifting from conduction to keyhole mode melting. 
CHAPTER 9 A STUDY OF TRANSIENT AND STEADY-STATE REGIONS FROM SINGLE-TRACK DEPOSITION IN LASER POWDER BED FUSION

\subsection{Introduction}

Laser powder bed fusion (L-PBF) is a powder bed additive manufacturing (AM) process that uses a laser to melt the powder layers selectively. The laser moves back and forth to form a layer, and the process is continued layer upon layer until a desired 3D part is produced. As a line scan is the basis of the SLM process, the effect of process parameters on its characteristics should be thoroughly investigated. In this regard, singletrack experiments have been performed with different materials like stainless steel grade 316L [82], IN625 [62], Ti-6Al-4V [87, 107], AlSi10Mg [70], etc.

The single-track experiments can help reduce the number of multiple layer experiments required to obtain the optimum process parameters. Knowing about the formation process of the single tracks, and their geometrical dimensions depending on the available powder would help obtain higher energy and powder efficiency [112]. Yadroitsev et al. [82] emphasized the significance of single-track experiments in the LPBF process. The single tracks from metal powders showed the stability and instability zones. The instabilities may appear at low scanning speed in the form of distortions or irregularities and at high-speed in the form of balling. Li et al. [61] characterized the 
balling formation in stainless steel and pure nickel powder and divided the balling into two types: ellipsoidal balls and spherical balls. Mathews et al. observed the denudation of metal powders near the laser scan path, which depend on laser parameters and ambient gas pressure [69]. Such a phenomenon would deplete the metal powders in the zone immediately surrounding the solidified track. Gunenthiram et. al [124] captured a highspeed video to analyze the formation of dynamic instabilities of the melt pool. The video and postmortem of the bead and powder bed explained the microparticles and macro spatters formation, and powder denudation effects. The authors concluded that the vaporization effects located near the laser-melt-pool interaction are the main driving force for most of these phenomena. Bidare et al. [125] used high-speed imaging and schlieren imaging to study the effects of interaction between the laser beam and the powder bed. The change in laser power and scan speed shifted the laser plume inclination, which resulted in different denudation regimes. It is also important to understand the formation of vapor depression during the laser-metal interaction. Hence, Cunningham et. al [95] used ultrahigh-speed synchrotron x-ray imaging to observe and quantify the vapor depression formed during the LPBF process. A clear transition from the conduction mode melting to keyhole mode melting was observed with an increase in the energy density. These studies show the complexity of the laser-material interaction during the L-PBF process. Nonetheless, the single track formed can be sub-divided into three regions: initial transient region during laser turn on, quasi-steady-state, and final transient region during laser turn off [84].

Numerical modeling is an important tool to understand the heat and mass transfer during laser-material interaction. For the single-track study, it is important to incorporate 
the powder particle distribution as it would affect the laser absorption and fluid flow [64]. Besides, different process physics such as thermo-capillary effect, recoil pressure, laser reflections, and absorption, etc. are important to accurately predict the melt pool. Khairallah et. al [64] developed a multi-physics model to predict heat transfer, fluid flow, and pore formation during single track formation. They emphasized the importance of including the Marangoni effect, recoil pressure to predict the surface formation. Panwisawas et. al [63] developed a numerical model to observe the changes in the thermal-fluid flow at different layers, and Shrestha and Chou [107] developed a numerical model to understand the physics behind the keyhole pore formation. These studies helped understand the process in detail which was difficult through experimentation alone. However, such high-fidelity models are computationally intensive and are limited to short-length simulation. It is observed that there is a huge variation in track width and bead height due to the transient nature at the beginning and end of the laser scan [84].

Bertoli et. al [84] explained that the transverse track geometry depends greatly on the location of the cross-section along the track. Besides, the height profile shows the bump in the initial transient region, and at the laser turn off point the height profile slopes downward with the increasing position. Moreover, the average height of the single track may be significantly higher than the layer thickness [103]. Such characteristics of the single tracks may increase the surface roughness and lead to mechanical interaction between the part and recoater blade during the powder spreading process, which is undesirable. The objective of this study is to observe and understand the formation of the transient and steady state region during the single-track scanning. For this purpose, $1 \mathrm{~mm}$ 
and $2 \mathrm{~mm}$ single tracks are fabricated using IN625, and the surface profiles of the single tracks are measured with the white light interferometer. The transient and steady-state zones are separated based on the track width and height. Besides, longitudinal metallography is performed to determine the relationship between different zones and the melt pool depth. In addition, a powder scale numerical model is developed to understand the physics that leads to the formation of the transient and steady-state regions during the single-track fabrication.

\subsection{Experiment Approach}

In this study, EOS M270 is used to fabricate $1 \mathrm{~mm}, 1.5 \mathrm{~mm}$, and $2 \mathrm{~mm}$ long IN625 single tracks with $195 \mathrm{~W}$ laser power and scan speeds of $400 \mathrm{~mm} / \mathrm{s}$, and 800 $\mathrm{mm} / \mathrm{s}$. The layer thickness of $40 \mu \mathrm{m}$ is used, and the single tracks are formed on top of the semi-cylinder base. The single tracks are formed $5.6 \mathrm{~mm}$ above the build plate, which include $3.1 \mathrm{~mm}$ support and $2.5 \mathrm{~mm}$ semi-cylinder radius. Figure 9.1 shows the top view of the single tracks. The single tracks are fabricated with the user parameters, while the semi-cylinder base is fabricated using the default EOS parameters. Besides, the skywriting setting is enabled to ensure that the acceleration and deceleration of the laser occur outside of the scan track, and constant speed is maintained during the single-track formation.

The track profiles are measured using a white light interferometer and the transient and steady regions are separated based on the track width and height. Besides, the metallography is performed to obtain the longitudinal melt pool boundary at the 
center of the track and observe the variation in the depth at the transient and steady region.

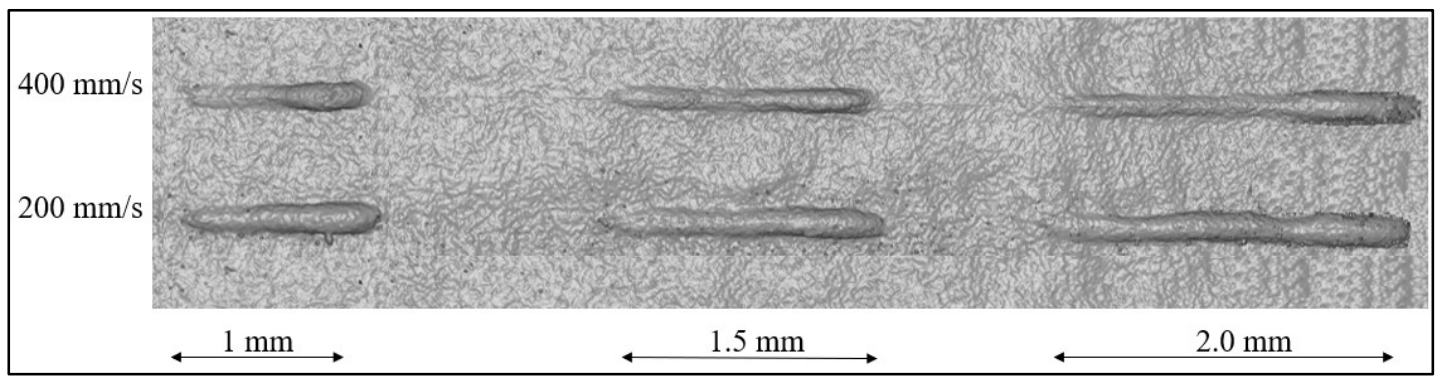

Figure 9.1 Single tracks fabricated on top of the semi-cylinder base.

\subsection{Numerical Approach}

\subsubsection{Discrete element method}

An opensource discrete element method (DEM) code called LIGGGHTS is used to simulate the powder spreading process and obtain a layer of powder [107]. During the powder spreading process, it is important to include the cohesion energy density of the particles [126]. However, the IN625 powder cohesion energy has not been studied before. Hence, a flowmeter setup is designed based on the literature [126] to calibrate the cohesion energy density for IN625. The design of the miniature flowmeter and the experimental setup is shown in Fig. 2. The setup consists of three major components: the flowmeter, a manual precision linear translation stage, and a $2.5 \mathrm{~mm}$ diameter base. The flowmeter is attached to the linear stage and is initially kept in contact with the base. As the contact between the flowmeter and base prevents the outward flow of the powder, IN625 powder particles are poured into the flowmeter. After the flowmeter is filled with the IN625 powder, the linear stage is moved in a positive Z-direction, that is away from 
the base. The powder flows out the flowmeter and collects at the stationary base as shown in Figure 9.2. The process is continued until the powder overflows. Finally, the angle of repose is measured from the powder settled on top of the cylinder base. Figure 9.3(a) shows the measurement of the angle of repose from the experiment. The experiment is repeated for 5 times to measure the repeatability of the angle of repose. The angle of repose is within $47 \pm 3^{\circ}$ based on all the measurements and accounting for the measurement uncertainty.

The powder size distribution used in the experiment is used in the simulation to calibrate the DEM model. Discrete powder size distribution between $18 \mu \mathrm{m}$ and $48 \mu \mathrm{m}$ is considered in the simulation. The angle of response during the flowmeter experiment is used as the calibration parameter. The flowmeter design with the same dimension is modeled to perform the DEM simulation. A modified simplified Johnson-KendallRoberts (SJKR2) cohesion contact model is used to include the cohesion effect. The SJKR2 model adds the normal force contribution when the particles are in contact.

$$
\mathrm{F}=\mathrm{kA}
$$

where, $\mathrm{k}$ is the cohesion energy density in $\mathrm{J} / \mathrm{m}^{3}$, and A is the particle-particle contact area. The cohesion energy density of $10^{6} \mathrm{~J} / \mathrm{m}^{3}$ resulted in approximately $47^{\circ}$ angle of repose in the simulation as shown in Figure 9.3(b). Besides the cohesion energy density, other properties used in the simulation are listed in Table 9.1. 

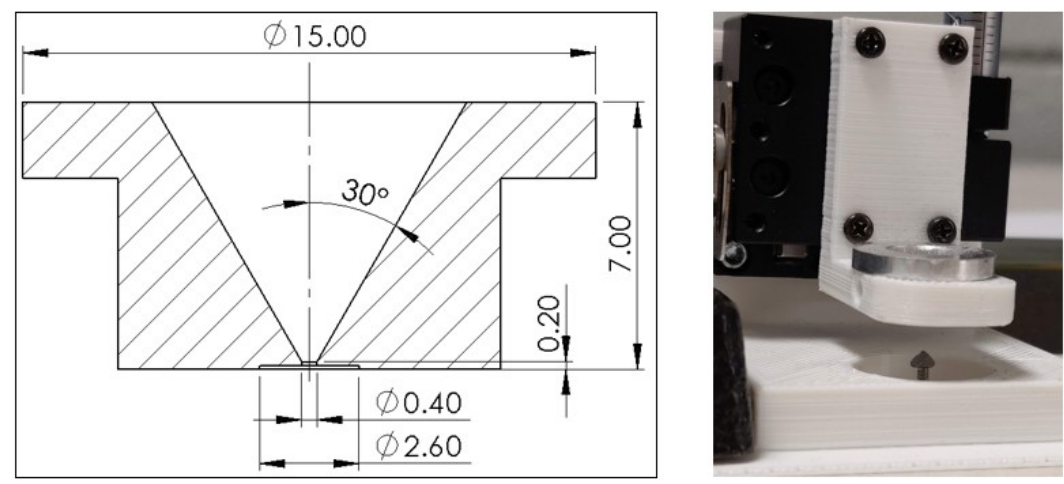

Figure 9.2 Design and dimensions of the miniature flowmeter and the setup used to obtain the angle of repose.

(a)

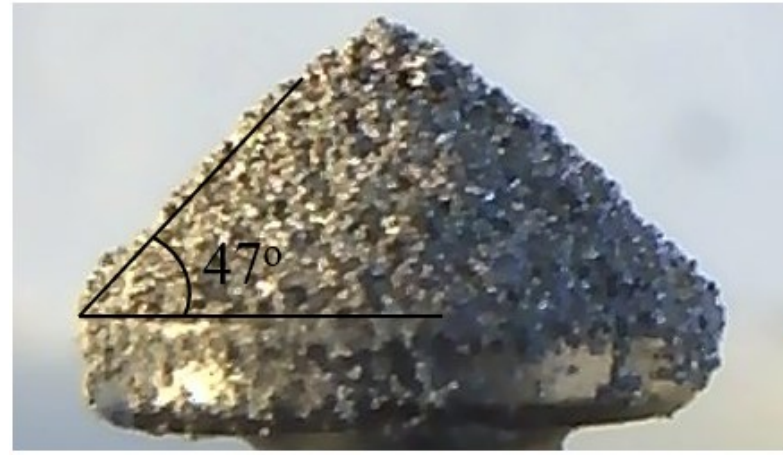

(b)

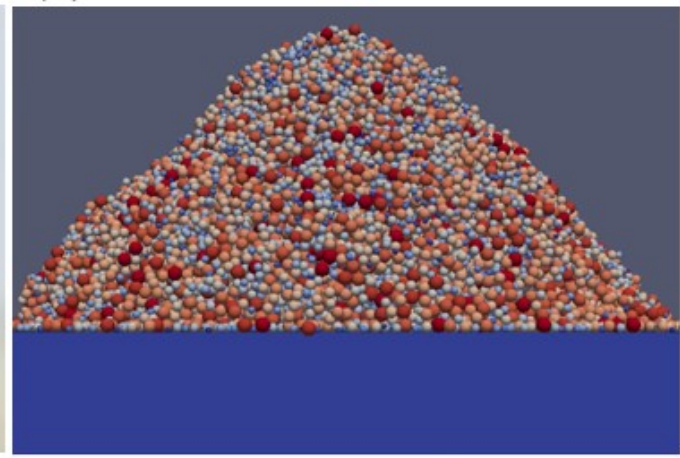

Figure 9.3 (a) Experiment and of repose used to calibrate cohesion energy density in (b) DEM simulation.

Table 9.1 . Inconel 625 properties for DEM simulation.

\begin{tabular}{ll}
\hline Parameters & Values \\
\hline Density $\left(\mathrm{Kg} / \mathrm{m}^{3}\right)$ & 8440 \\
Modulus of Elasticity $(\mathrm{GPa})$ & 200 \\
Poisson's ratio & 0.3 \\
Coefficient of restitution & 0.5 \\
Sliding friction coefficient & 0.5 \\
Rolling friction coefficient & 0.1 \\
\hline
\end{tabular}

Figure 9.4 shows the simulation domain used to simulate the powder spreading process. The powder size distribution used in the calibration of cohesion energy density 
are generated based on the volume ratio and dropped into the dispenser platform. After the desired volume of particles is generated, the particles are let to settle on the dispenser container. Once settled, the dispenser platform is moved upwards towards the recoater blade. Finally, the recoater is moved to spread a layer of powder over the build platform as shown in Figure 9.4. In this study, the gap distance of $80 \mu \mathrm{m}$ is used considering the increase in the actual powder layer thickness after certain layers during the L-PBF process [127].

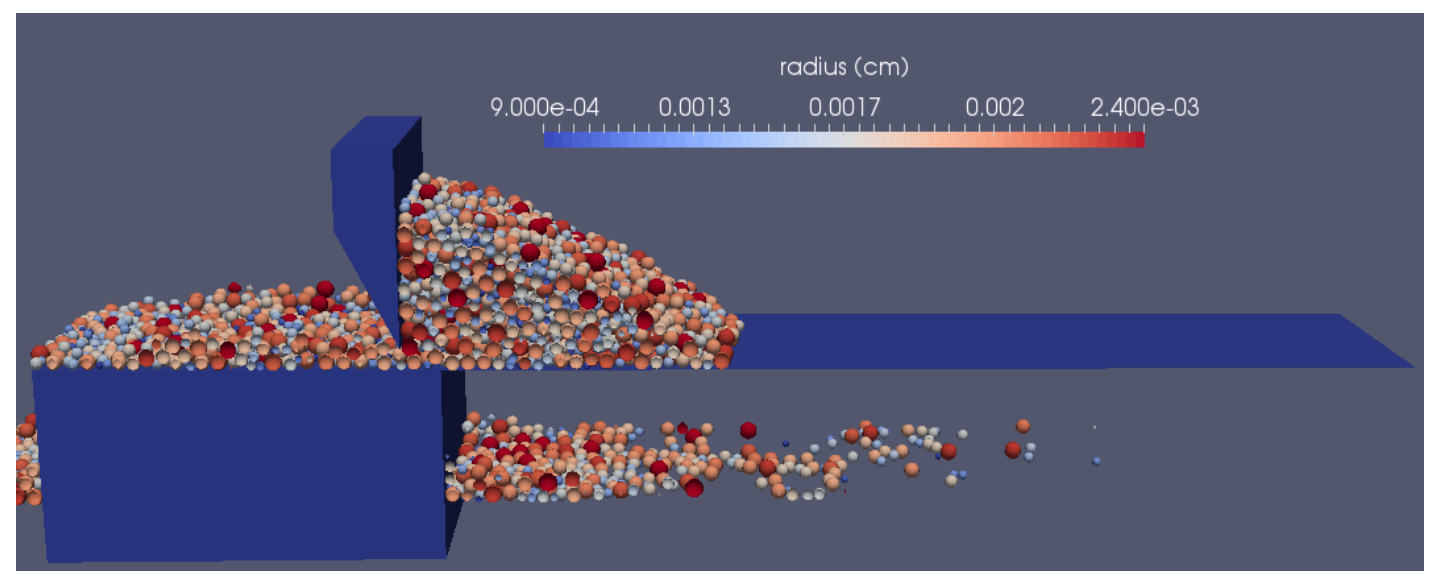

Figure 9.4 DEM setup to spread a layer of powder.

\subsubsection{Powder scale thermo-fluid model}

FLOW-3D commercial simulation software is used to develop a high-fidelity numerical model. Figure 9.5 shows the simulation domain used in this study. The powder layer obtained from the DEM simulation is defined over the solid substrate. The volume of fluid (VOF) method is used to track the free surface, and the materials are distinguished based on the fluid fraction. The solid substrate and powder have a fluid fraction of 1 , and the inert gas region over the powder has a fluid fraction of 0 . Material properties of IN625 are assigned to the regions with a fluid fraction of 1, which is powder 
and solid substrate. The temperature-dependent material properties of IN625 used in the simulation are shown in Figure 9.6, and other properties are listed in Table 2. The boundary conditions such as the laser profile at the free surface, pressure boundary conditions at the fluid interface, etc. have been discussed in the previous study [4].

Single tracks with scan lengths of $1 \mathrm{~mm}$ and $2 \mathrm{~mm}$ are simulated using laser power of $195 \mathrm{~W}$ and scan speeds of $400 \mathrm{~mm} / \mathrm{s}$ and $800 \mathrm{~mm} / \mathrm{s}$. The laser scan is started from $0.25 \mathrm{~mm}$ in X-direction, which continues up to $1.25 \mathrm{~mm}$ for a $1 \mathrm{~mm}$ track and up to $2.25 \mathrm{~mm}$ for a $2 \mathrm{~mm}$ track. The depth of the substrate is increased from $0.2 \mathrm{~mm}$ to 0.3 $\mathrm{mm}$ for $195,400 \mathrm{~mm} / \mathrm{s}$ due to an increase in the energy density. Hence, the domain size used for $195,400 \mathrm{~mm} / \mathrm{s}$ is $2.5 \mathrm{~mm} \times 0.4 \mathrm{~mm} \times 0.42 \mathrm{~mm}$.

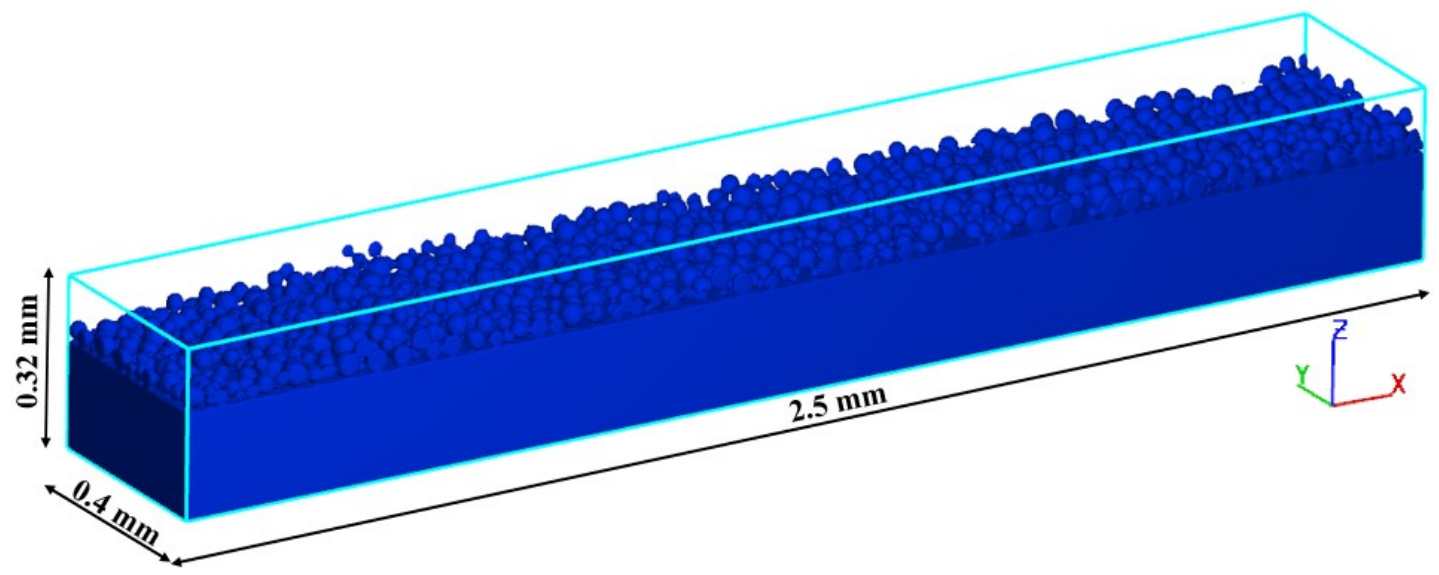

Figure 9.5 Simulation domain used for $195 \mathrm{~W}$ and $800 \mathrm{~mm} / \mathrm{s}$.
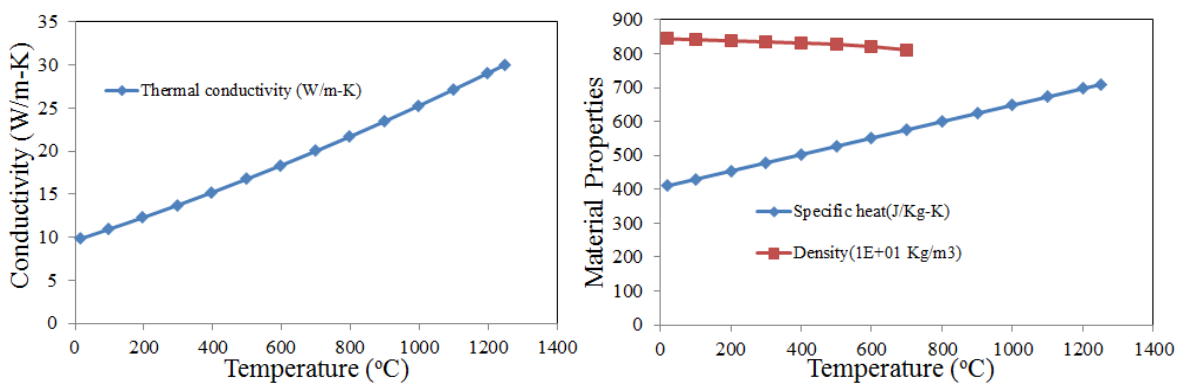

Figure 9.6 Temperature dependent material properties of Inconel 625[18]. 
Table 9.2 Properties of In625 used in a thermo-fluid simulation.

\begin{tabular}{ll}
\hline Parameters & Values \\
\hline Solidus temperature, $T_{S}(\mathrm{~K})$ & 1563 \\
Liquidus temperature, $T_{L}(\mathrm{~K})$ & 1623 \\
Boiling temperature, $\mathrm{T}_{\mathrm{v}}(\mathrm{K})$ & 3188 \\
Viscosity $(\mathrm{kg} / \mathrm{m}-\mathrm{s})$ & 0.007 \\
Surface tension $(\mathrm{N} / \mathrm{m})$ & 1.8 \\
Surface tension gradient $(\mathrm{N} / \mathrm{m}-\mathrm{K})$ & $-2 \mathrm{E}-05$ \\
\hline
\end{tabular}

9.4 Results and Discussion

9.4.1 Experimental results

White light interferometer

Figure 9.7(a) presents the surface morphology of the single-track formed with 195 $\mathrm{W}$ and $800 \mathrm{~mm} / \mathrm{s}$. At the start of the scan, a wider track with bump is noticed. The longitudinal (X) profile in Figure 9.7(b) shows that the height of the track decreased along the scan direction. The higher track at the start may be due to the strong backflow of the melt pool. Besides, the maximum height of the bead is around $100 \mu \mathrm{m}$ which is significantly higher compared to the layer thickness of $40 \mu \mathrm{m}$. Such an increase in height may be due to the movement of the neighbor powder particles into the melt pool [69]. Moreover, the actual layer thickness is 4-5 times the defined layer thickness due to the inherent shrinkage of the previous layers and the powder dynamics [127]. Besides, the end of the track has powder particles attached to it, which increases the surface height in the longitudinal profile shown in Figure 9.7(b).

The transverse surface (Y) profile, which is shown in Figure 9.7(b), can be used to measure the width of the track. The width of the track is around $150 \mu \mathrm{m}$ from the 
obtained profile. However, there is a huge variation in the width along the track. Hence, the formation of the steady region is not evident with the $1 \mathrm{~mm}$ long track, which suggested that the longer scan lengths need to be analyzed. Therefore, the surface profile of a $2 \mathrm{~mm}$ track is obtained to observe the transition from a transient zone to a steady zone.
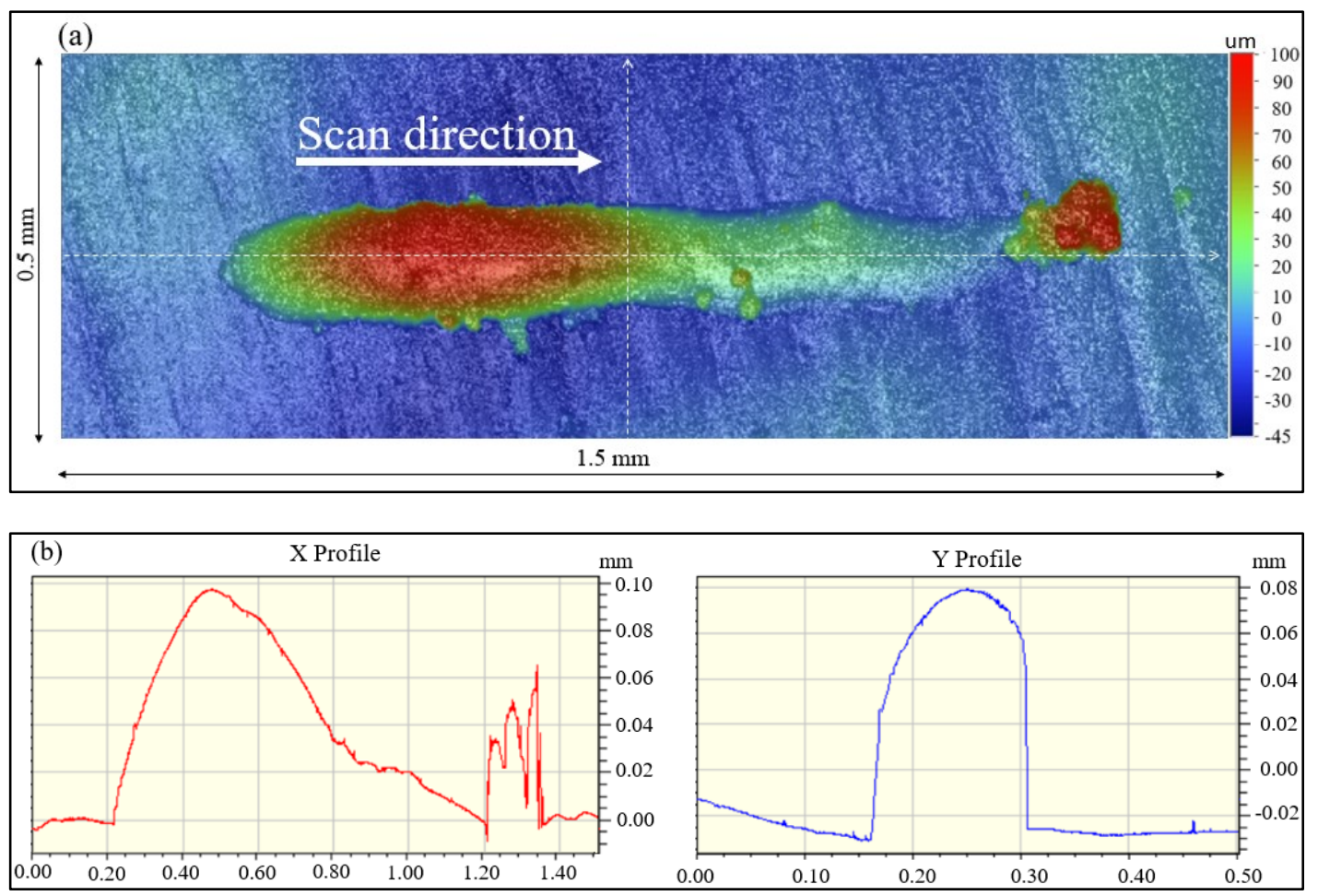

Figure 9.7 (a) Surface profile, and (b) longitudinal and transverse 2D profile of $1 \mathrm{~mm}$ single track formed with $195 \mathrm{~W}$ and $800 \mathrm{~mm} / \mathrm{s}$.

Figure 9.8 presents the surface morphology of a $2 \mathrm{~mm}$ track formed with $195 \mathrm{~W}$, $800 \mathrm{~mm} / \mathrm{s}$, and the 2D longitudinal profile. Three zones may be identified based on the width of the track. The initial transient region is wider and has a higher variation in track width, the steady-state region has almost constant track width, while the final transient region has a decreasing track width. Although the regions are identified based on the 
track width, the $2 \mathrm{D}$ profile shows that the regions may not be separated based on the surface height. The height of the track decreased gradually along the scan direction.

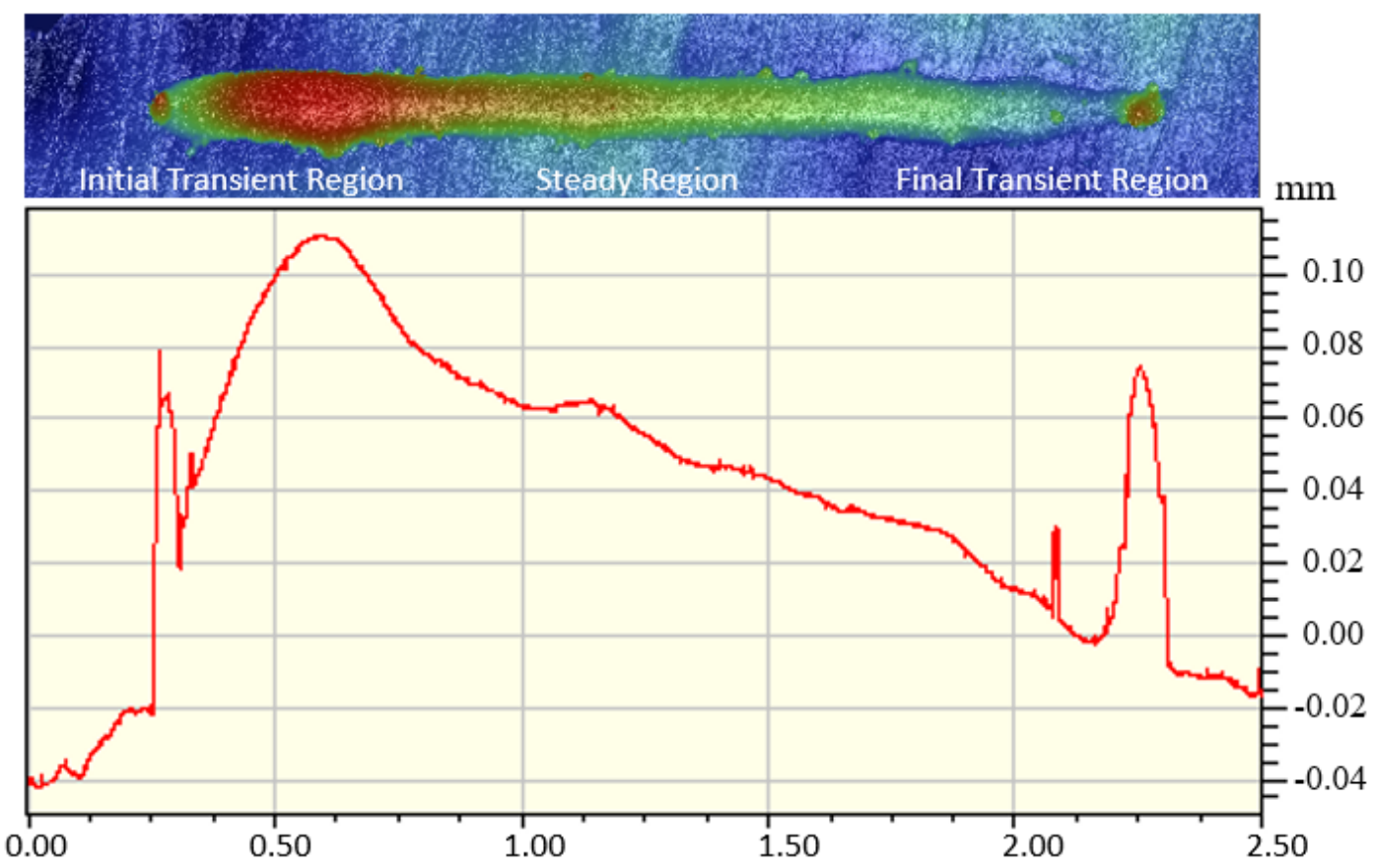

Figure $9.82 \mathrm{~mm}$ single track surface profile and longitudinal 2D profile formed with 195 $\mathrm{W}$ and $800 \mathrm{~mm} / \mathrm{s}$ showing three zones.

Figure 9.9(a) shows the surface morphology of a $1 \mathrm{~mm}$ long single-track formed with $195 \mathrm{~W}$ and $400 \mathrm{~mm} / \mathrm{s}$. The track width gradually decreased from the beginning of the scan to the end as observed for the single track formed with $195 \mathrm{~W}$ and $800 \mathrm{~mm} / \mathrm{s}$. Figure 9.9(b) presents the longitudinal (X profile) and transverse (Y profile) taken at the line shown in Figure 9.9(a), which again showed the similarity to the $195 \mathrm{~W}, 800 \mathrm{~mm} / \mathrm{s}$ case too. However, the track width is higher in this case $(180 \mu \mathrm{m})$ due to the increase in energy density. Again, it is apparent that the melt pool did not reach the steady state during a $1 \mathrm{~mm}$ scan. 

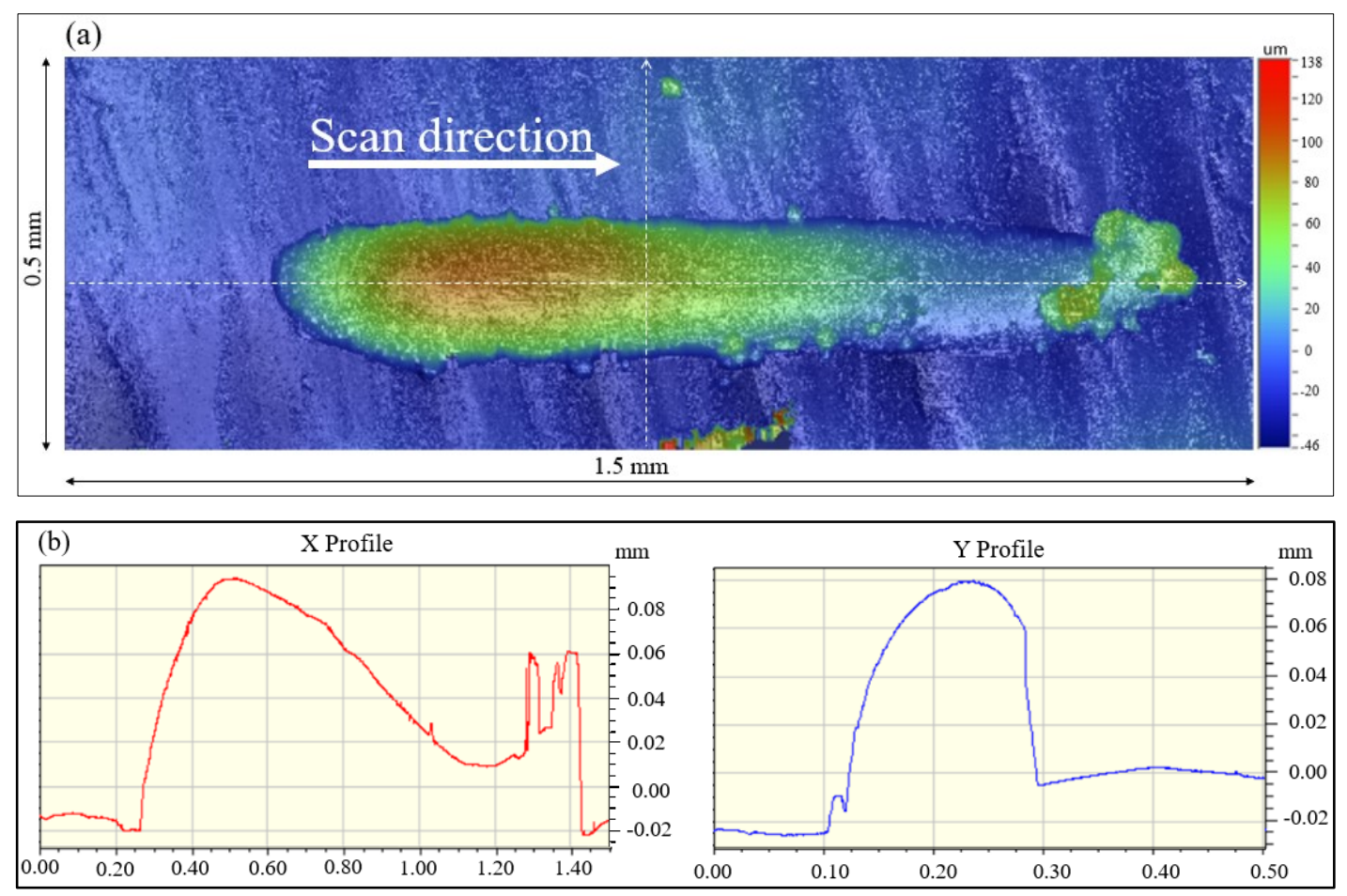

Figure 9.9 (a) Surface profile, and (b) longitudinal and transverse 2D profile of $1 \mathrm{~mm}$ single track formed with $195 \mathrm{~W}$ and $400 \mathrm{~mm} / \mathrm{s}$.

Figure 9.10 exhibits the surface morphology of a $2 \mathrm{~mm}$ long single track along with the $2 \mathrm{D}$ longitudinal profile. In this case, a steady region is observed, and two parallel white dash lines are drawn on the top view based on the central steady region. These lines help to identify the transient zones, and the single track is divided into three regions based on the track width. The initial transient state is defined where a bump is formed, and the track width is higher compared to other regions. A middle steady-state is the region where the height and width are more consistent. Finally, at the end of the track, where the laser turns off, the bead height is very small along with a tapered melt profile. It is difficult to establish distinctive criteria to separate the three zones, although the existence of transient and steady-state zones is obvious. Hence, an approximation is made 
to separate the three zones, and the approximate transient length at the beginning is approximately $400 \mu \mathrm{m}$.

As in all other cases, the powder particles are observed at the end of the track. During the laser melting, the induced flow of the ambient gas entrains the powder particles towards the melt pool[125]. During the laser turn off, the consolidated powder particles may get adhered to the solidifying melt pool. Besides the end of the track, powder particles are also observed in the other regions.

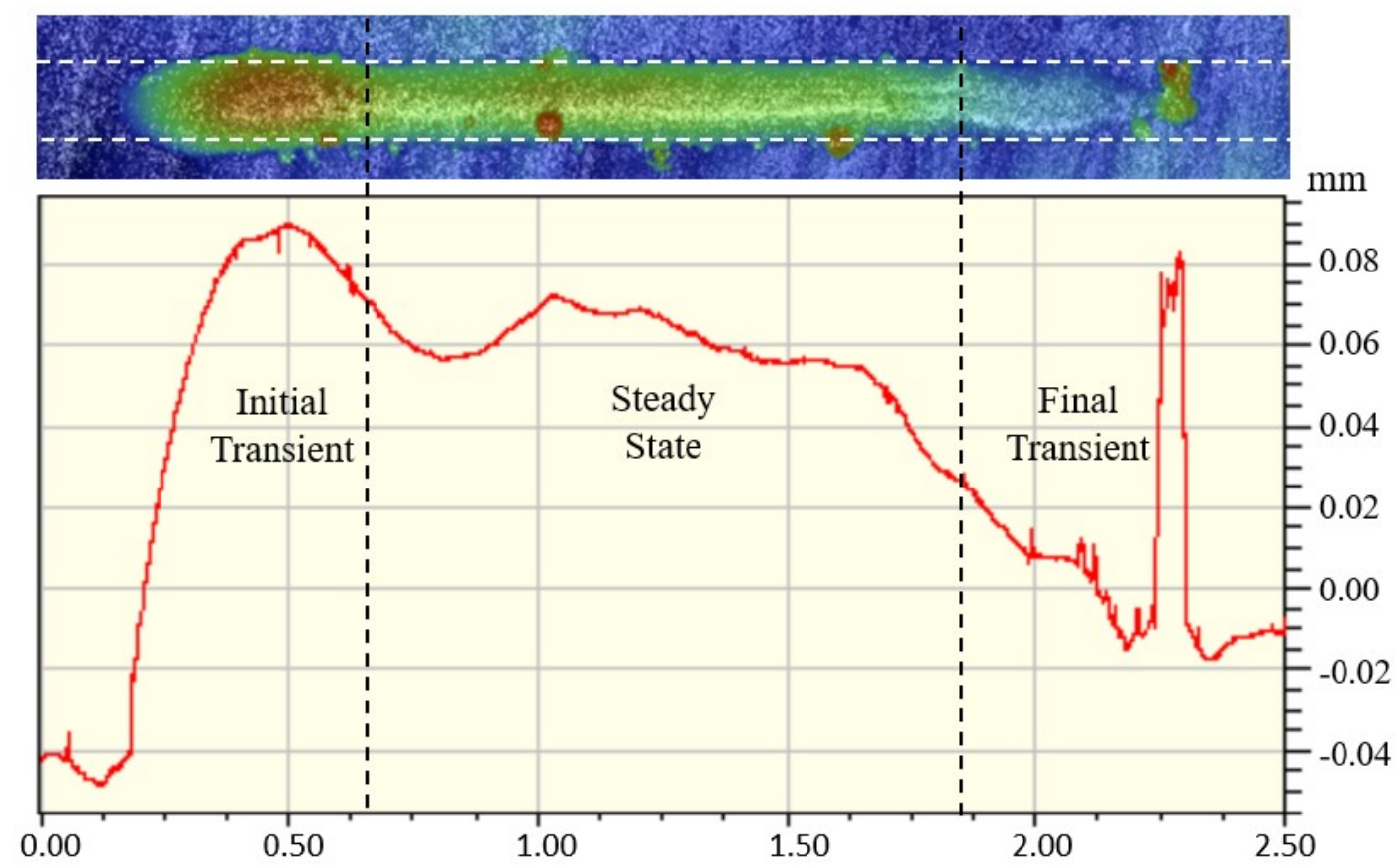

Figure $9.102 \mathrm{~mm}$ single track surface profile formed with $195 \mathrm{~W}$ and $400 \mathrm{~mm} / \mathrm{s}$ showing three zones.

\section{Metallography}

WLI results showed the presence of transient regions at the beginning and end of the tracks. It is desired to know the effect of transient behavior on the melt pool depth. Hence, the metallography is performed to obtain the longitudinal melt pool boundary. 
The longitudinal micrograph of a $1 \mathrm{~mm}$ single track formed with $195 \mathrm{~W}$ and $800 \mathrm{~mm} / \mathrm{s}$ is shown in Figure 9.11. The depth varied from $60 \mu \mathrm{m}$ to $105 \mu \mathrm{m}$, with the maximum depth being at the initial region. The single tracks are formed over the LPBF fabricated base. Hence, the variation in the surface, over which the single track is fabricated, may amplify the variation in the depth of the melt pool. The longitudinal section showed that the initial region has higher bead height and deeper melt pool in this case. However, there is no distinct effect of the transient region on the melt pool depth.

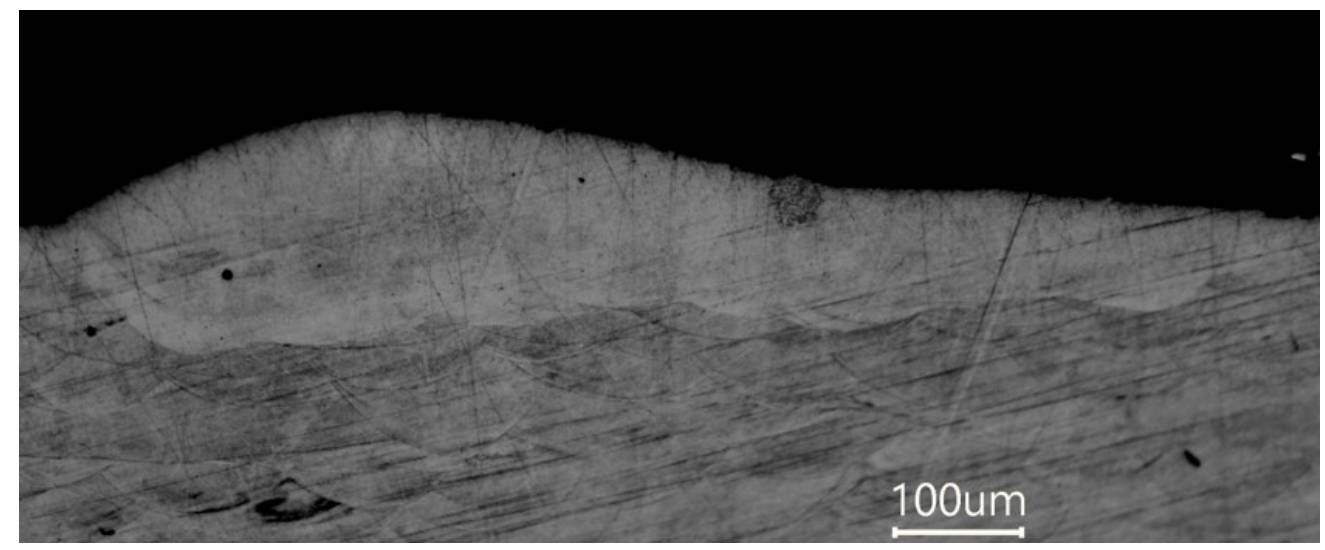

Figure 9.11 Longitudinal melt profile obtained with $195 \mathrm{~W}$ and $800 \mathrm{~mm} / \mathrm{s}$.

Figure 9.12 shows the longitudinal melt pool boundary of a $1 \mathrm{~mm}$ single track formed with $195 \mathrm{~W}$ and $400 \mathrm{~mm} / \mathrm{s}$. The variation in the depth is not significant compared to the depth in this case even though there is a huge variation in the surface profile. Besides, the initial region did not have a higher melt pool depth compared to other regions. Again, the single tracks are formed on top of the base formed with the L-PBF process as well, and the surface variation would affect the laser penetration during a single-track formation. In addition, a pore is formed at the beginning of the scan for both 
tracks. The metallography results obtained from $400 \mathrm{~mm} / \mathrm{s}$ and $800 \mathrm{~mm} / \mathrm{s}$ scan speeds indicated that the transient behavior of the melt pool did not affect the melt pool depth.

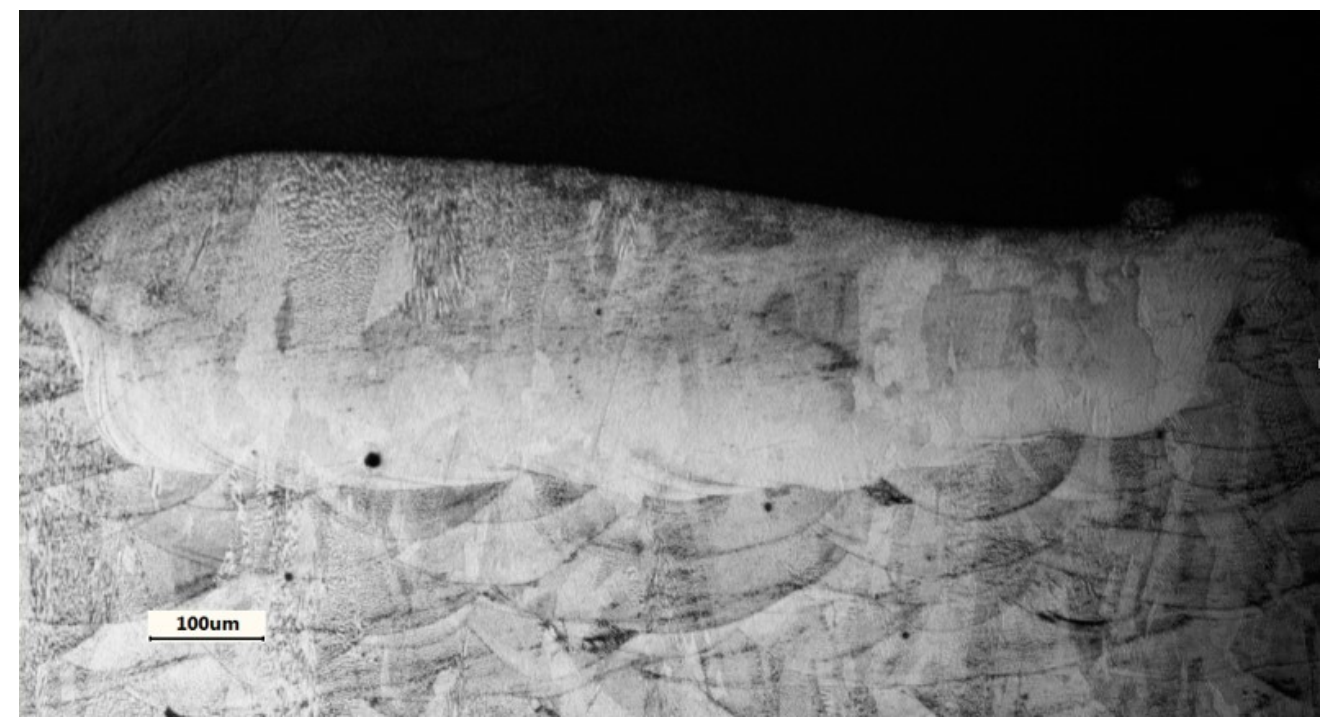

Figure 9.12 Longitudinal melt profile obtained with $195 \mathrm{~W}$ and $400 \mathrm{~mm} / \mathrm{s}$.

\subsubsection{Numerical Results}

The experimental results showed the presence of transient zones at the beginning and end of the scan and the steady-state zone in between the two transient zones. However, the underlying physics leading to such behavior is not understood with postexperimental analysis. The heat and mass transfer during the single-track scanning would drive the formation of transient regions. Hence, a high-fidelity material-based model is developed to understand the formation of transient and steady-state regions. The laser power of $195 \mathrm{~W}$ with scan speeds of $400 \mathrm{~mm} / \mathrm{s}$ and $800 \mathrm{~mm} / \mathrm{s}$ are used to predict the single-track formation during the L-PBF process and understand the physics behind the formation of transient and steady regions. 


\section{Conduction mode}

During the L-PBF process, the powder bed is scanned with laser. The powder particles and the substrate beneath the powder layer absorb the energy from the laser and immediately melts forming a melt pool. Figure 9.13 shows the development of the melt pool as the laser moves in a straight line. Before the laser scan, the powder bed is at the preheat temperature of $353 \mathrm{~K}$. As the laser turns on and starts melting the powder particles and the substrate, the temperature gradient around the melt pool is maximum due to the lower surrounding temperature. The melt flow occurs outward and backward because of the coupled effect of Marangoni flow and the recoil pressure. The recoil pressure pushes the melt pool out of the laser scan region, forming a depression, and the melt pool flows from higher temperature to lower temperature due to the negative surface tension gradient. In addition, the thermal conductivity of the powder and solid is lower when the laser first strikes the powder bed, which may lead to a wider melt pool at the beginning of the scan. As the laser travels, the thermal gradient becomes steady and the outward flow is lower compared to the beginning of the scan. This difference in flow behavior leads to the differences in the track width. 


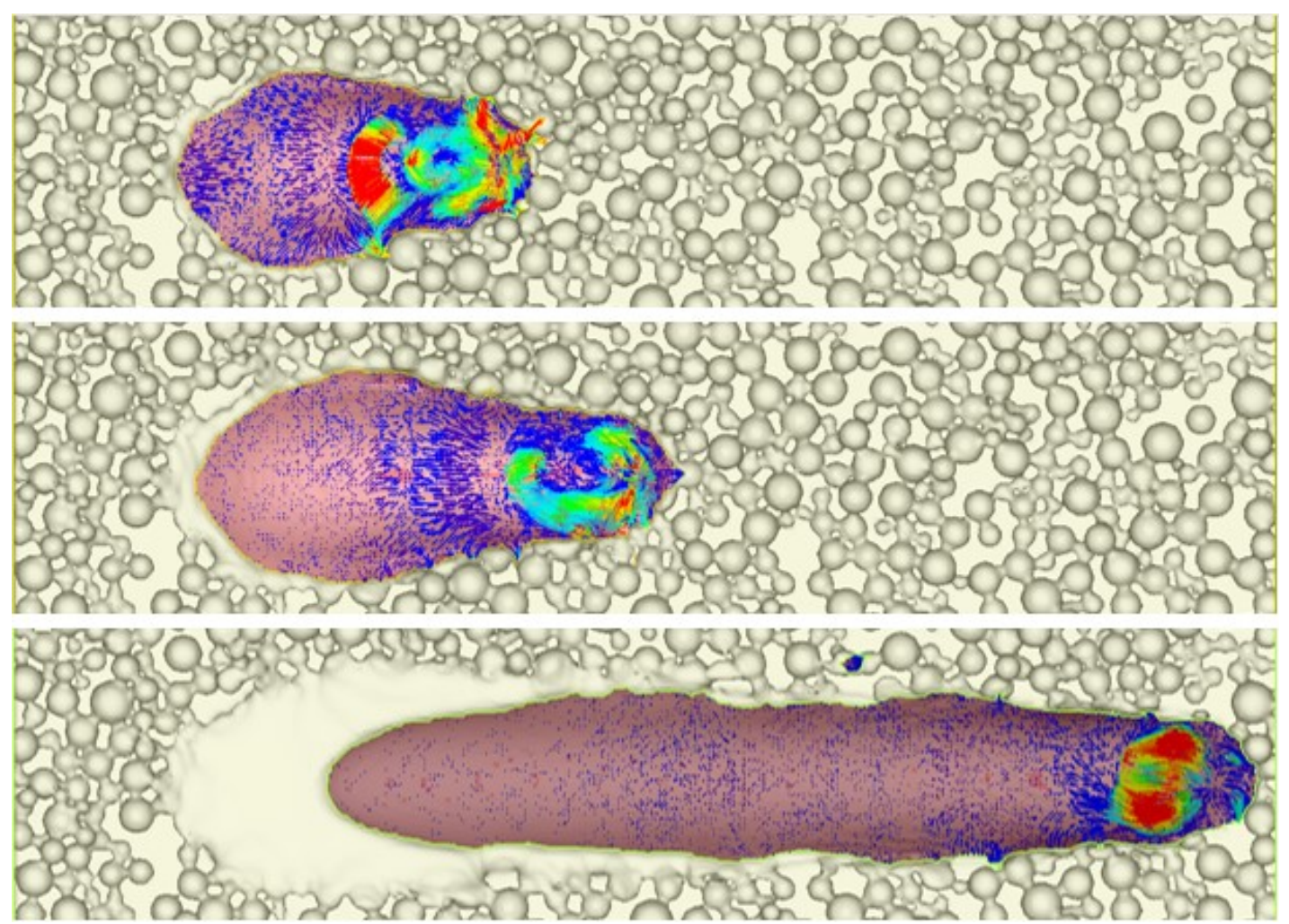

Figure 9.13 Melt flow during the single-track scanning process.

Figure 9.14 (a) presents the top view of the melt region, which shows a gradual decrease in the track width along the scan direction. As the melt pool length is in the range of $1 \mathrm{~mm}$, a steady-state region is not identified from the $1 \mathrm{~mm}$ scan length simulation. The top profile at the substrate level is extracted from the simulation and the melt profile is compared with the experiment in Figure 9.14(b). The simulation can predict the melt profile trend during a $1 \mathrm{~mm}$ simulation. As the shape of the melt pool has a narrow region in the laser application area, the end of the scan, where the laser turns off, has a tapered profile. The transient behavior of the single-track at the beginning and end of the scan track is due to the inherent heat and mass transfer characteristics during the L-PBF process. 
It is important to note the limitations of the simulation to predict the formation of the bead. The L-PBF process involves powder dynamics that highly affects the formation of the surface profile, which is not included in the simulation. Rather, the powder particles are fixed and do not flow due to the pressure difference generated in the melt pool and surrounding during the melting process.
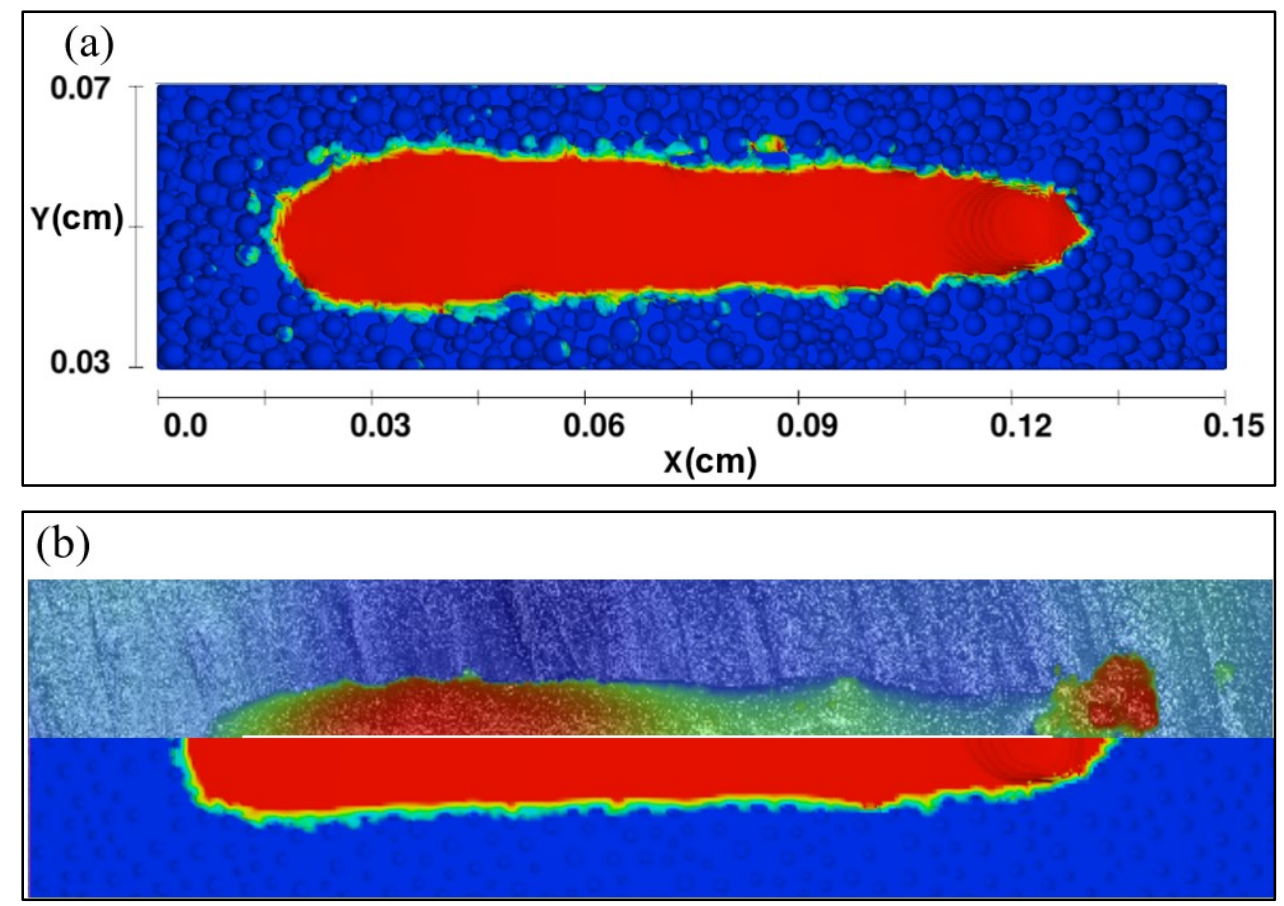

Figure 9.14 (a) Top view of the melt profile formed with $195 \mathrm{~W}$ and $800 \mathrm{~mm} / \mathrm{s}$ and (b) Track profile compared at the free surface between experimental and simulation.

Figure 9.15 shows the top view of the melt region obtained for a $2 \mathrm{~mm}$ scan length with $195 \mathrm{~W}$ and $800 \mathrm{~mm} / \mathrm{s}$. In this case, three regions are distinctly identified. The transient region during the laser turn-on shows higher widths compared to the steadystate region and the laser turn off region has the smaller melt pool width. Two parallel lines are drawn based on the steady region, which shows that the transient length at the beginning of the scan is approximately $400 \mu \mathrm{m}$. 


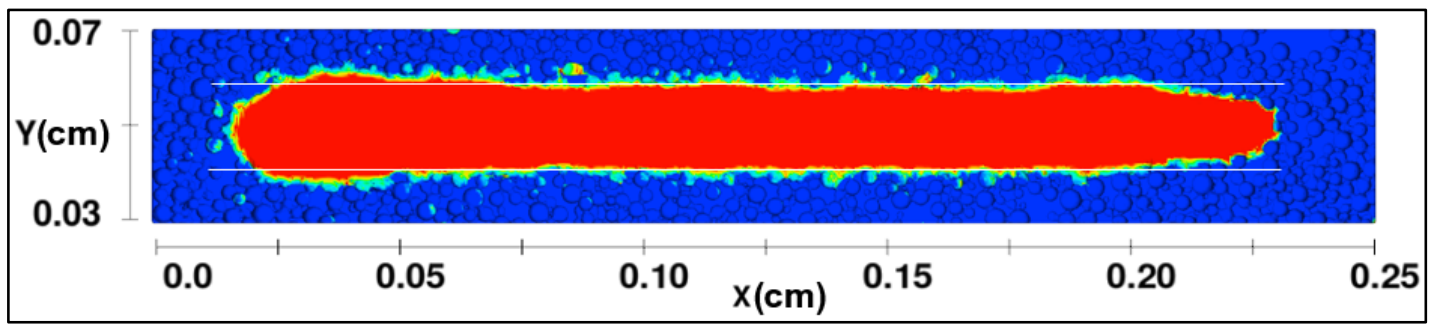

Figure 9.15 Top melt profile obtained for $2 \mathrm{~mm}$ simulation with $195 \mathrm{~W}$ and $800 \mathrm{~mm} / \mathrm{s}$.

Figure 9.16 illustrates the formation of the single track shown in Fig. 15 by presenting the temperature contour and melt pool formed at the interface between the substrate and powder. The powder and the substrate are maintained at $353 \mathrm{~K}$ initially. As the powder absorbs the laser energy, the temperature of the particles increases. The heat is then transferred to the surrounding particles and substrate through heat diffusion. A bigger melt region may form at the laser turn-on region due to the higher thermal gradient at the beginning and lower thermal conductivity of the surrounding. As the melting progresses, the recoil pressure created a depression zone within the melt pool, with most of the materials being pushed backward. This transport of material from the laser applied zone to the back of the melt pool would form an elevation region.

Figure 9.16 shows seven isotherms with temperatures ranging from melting temperature to the evaporation temperature. At the start of the scan, the distance between the isotherms are very similar, and the isotherms are elliptical. The distance between the successive isotherms' changes as the laser travels further. After some time, the isotherms have more pointed end at the solidifying region, which signals the formation of fully developed melt pool. The melt pool shape of the melt pool and the isotherms are consistent after the melt pool is fully developed. As the melt pool fully develops, the 
variation in the width of the melt pool is minimized. The melt pool stabilizes as the temperature gradient becomes steady. This region is defined as the steady-state zone. The shallow region is obtained at the end of the track when the laser turns off, since the melt flow is backward. Hence, the numerical simulation showed that the transient length at the beginning of the track may depend upon the material property, laser parameters, and the bed temperature. Moreover, the melt pool is narrower at the laser application area, and top view shows that the laser application area has semi-lemniscate shape. Hence, the track width decreases at the end transient region.

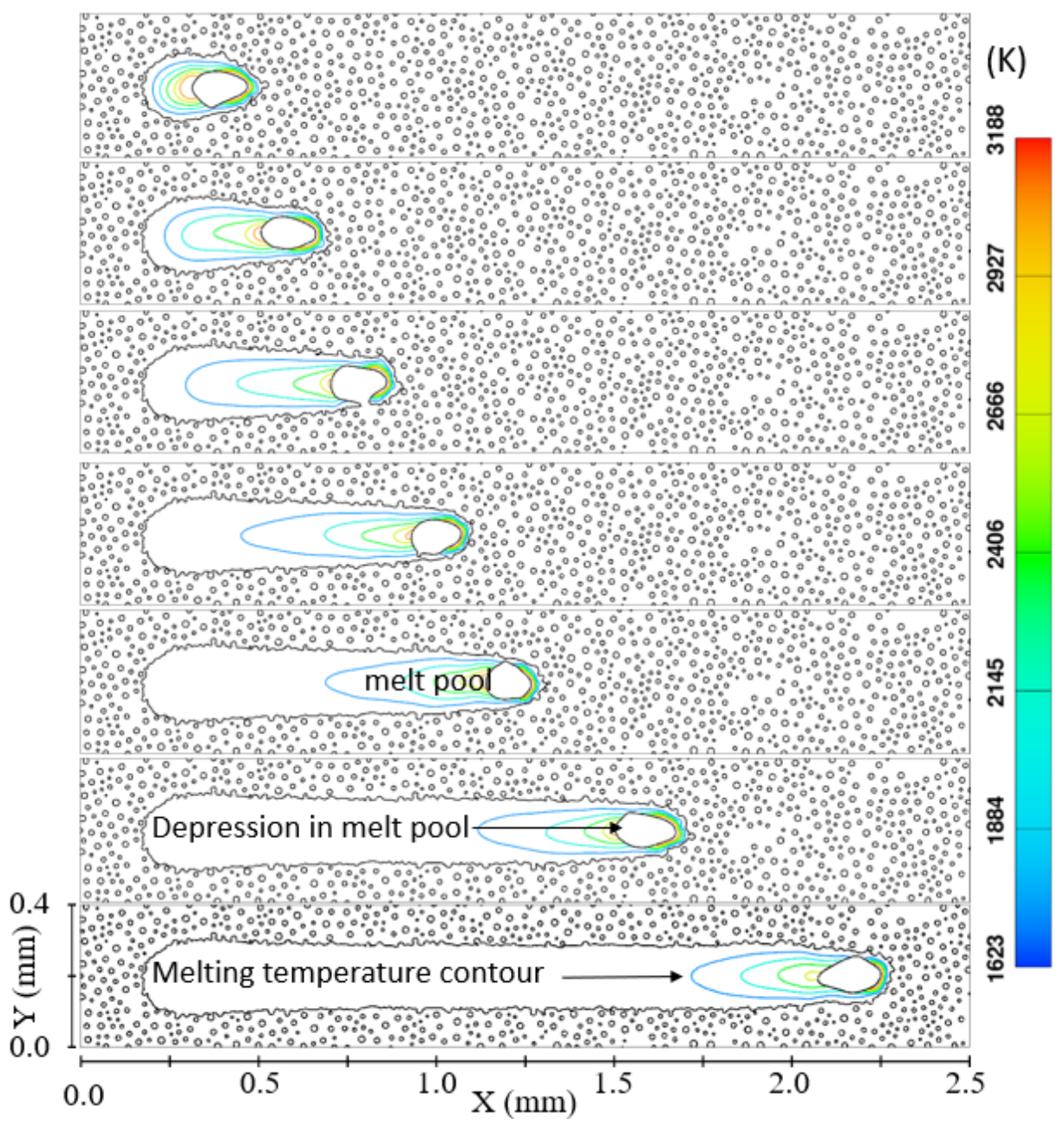

Figure 9.16 Development of melt pool: 2D temperature contours obtained at the interface between substrate and powder. 
Besides the top view, the transverse profile is compared. Figure 9.17 shows the experimental result and the simulation result. The transverse melt pool boundary is a function of the location as the longitudinal melt pool boundary showed huge variation in depth for $195 \mathrm{~W}$ and $800 \mathrm{~mm} / \mathrm{s}$. The specific 2D metallographic imaged used for the comparison shows the track width of $134 \mu \mathrm{m}$ and depth of $70.5 \mu \mathrm{m}$ while the simulation predicted the track width and depth of $140 \mu \mathrm{m}$ and $65 \mu \mathrm{m}$. The numerical model was not able to predict the depth variation along the longitudinal direction. One of the reasons may be the surface on which the powder is spread is flat in simulation. In the experiment, as the single track is formed on top of the previously deposition layer, there may be some variation in the surface and the powder which may incur inconsistency in the track depth. In addition, the single-track bead height is larger than the layer thickness used due to the added mass as a result of the powder influx to the melt pool from the neighbor, which is not captured by the simulation. In contrast to the experiment, the bead height in the simulation is lower than the layer thickness due to the shrinkage.
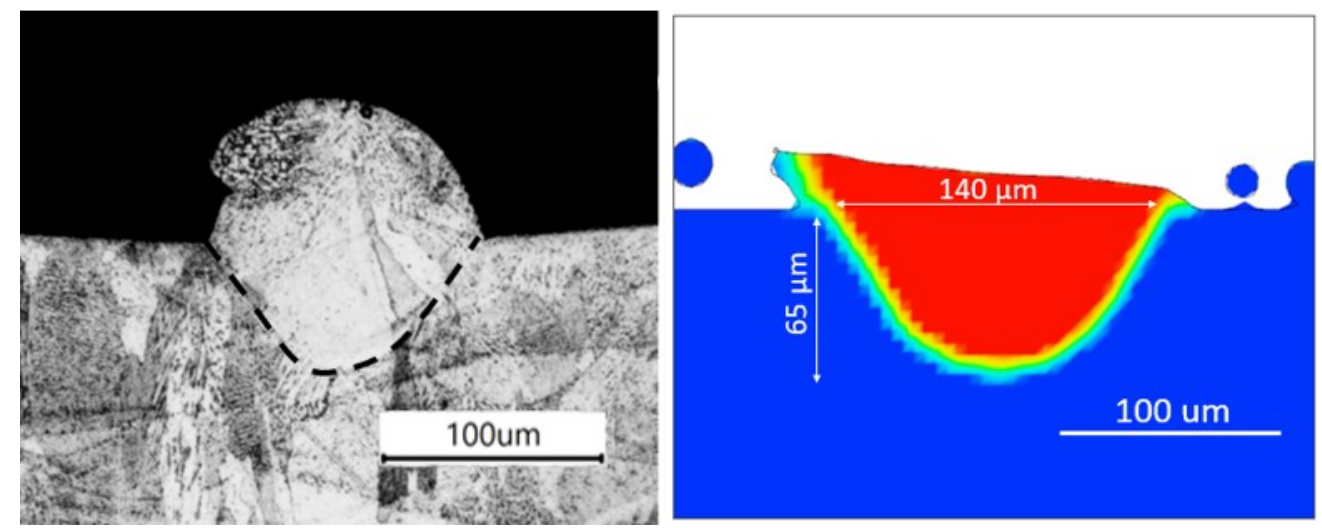

Figure 9.17 Transverse melt profile obtained for experiment and simulation with $195 \mathrm{~W}$ and $800 \mathrm{~mm} / \mathrm{s}$. 


\section{$\underline{\text { Keyhole mode }}$}

The single-track experiment results with $195 \mathrm{~W}$ and $400 \mathrm{~mm} / \mathrm{s}$ showed that keyhole is formed with this parameter. The simulation is carried out to predict the formation of $1 \mathrm{~mm}$ and $2 \mathrm{~mm}$ scan length. Figure 9.18 shows a comparison between the top profile between experiment and simulation. With the same power of $195 \mathrm{~W}$ and lower speed, the top profile showed less variation from the beginning of the track to the end, although the track width decreased along the laser travel direction.

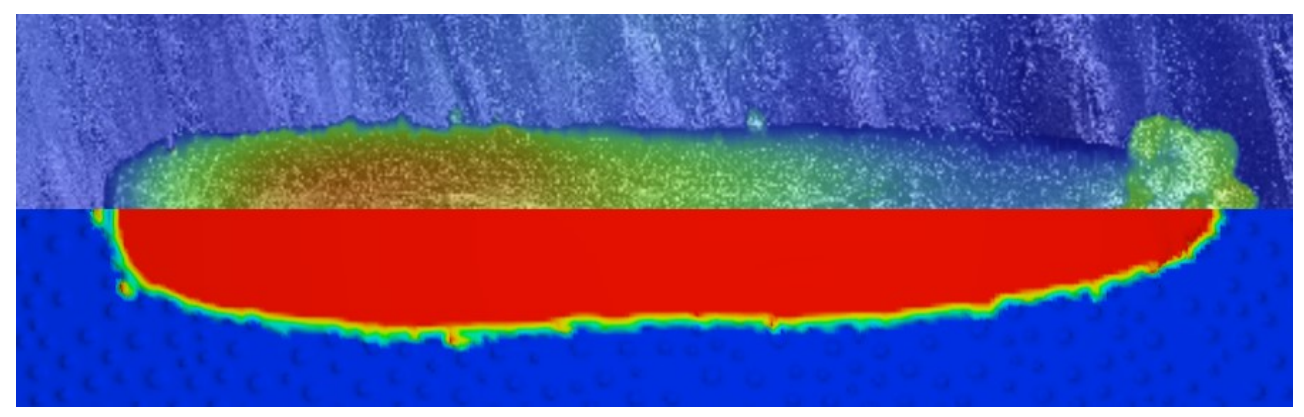

Figure 9.18 Track profile compared at the free surface between experimental and simulation single track formed with $195 \mathrm{~W}$ and $400 \mathrm{~mm} / \mathrm{s}$.

The development of the melt pool is analyzed for the $2 \mathrm{~mm}$ simulation case, and is very similar to $195 \mathrm{~W}, 800 \mathrm{~mm} / \mathrm{s}$ case. Fig. 9.19 shows the shape of the melt pool at different laser locations. The isotherms at the start of the scan are very close to each other, which leads to the formation of the transient region. As the laser moves, the size of the isotherms increases and becomes stable after the laser has traveled a certain distance. The melt pool fully develops only after traveling almost $1 \mathrm{~mm}$ as that in $800 \mathrm{~mm} / \mathrm{s}$ case. However, the radius of the depression opening, in this case, is small initially, which grows as the laser travels. 


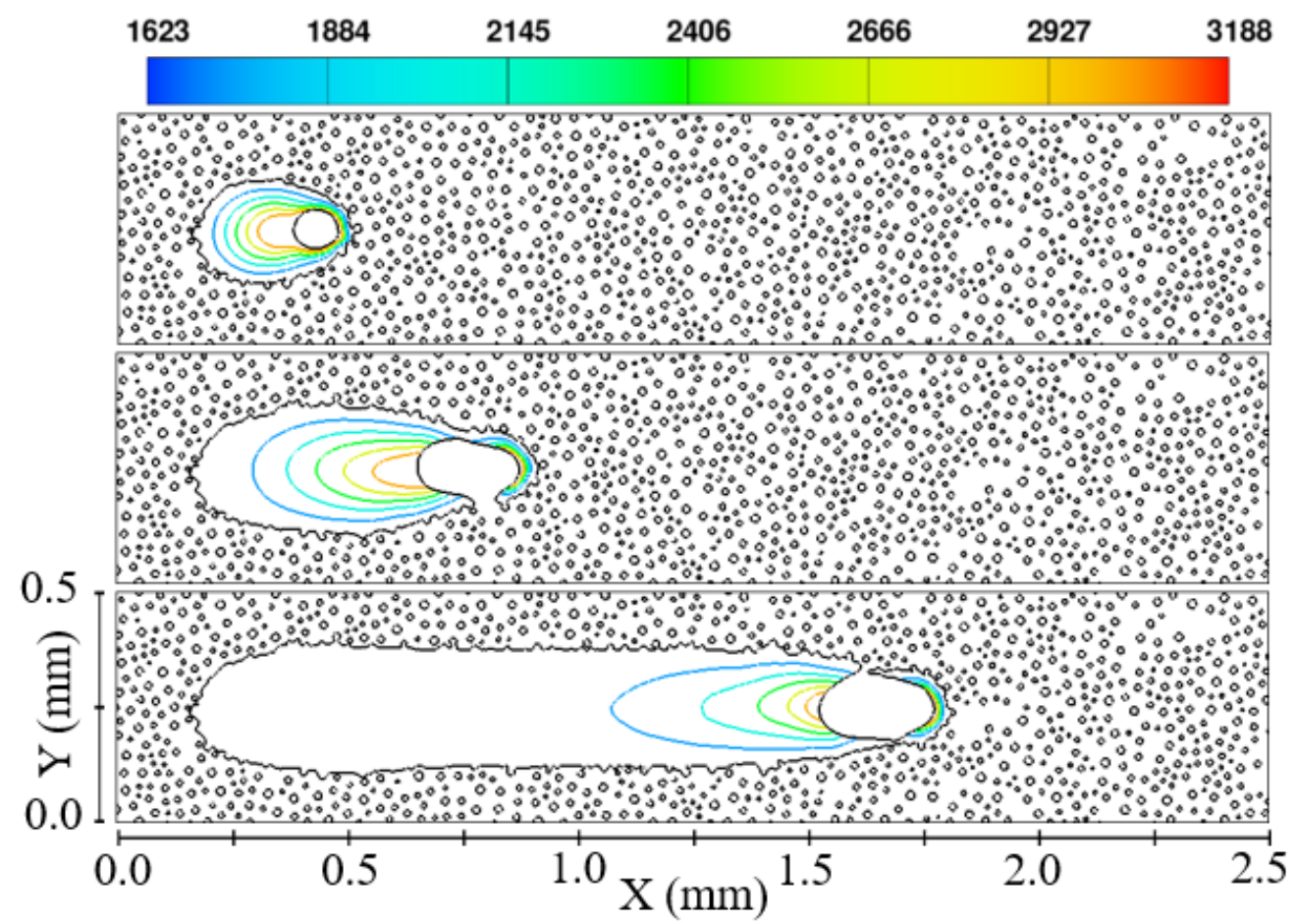

Figure 9.19 Development of melt pool: 2D temperature contours obtained at the interface between substrate and powder for $195 \mathrm{~W}$ and $400 \mathrm{~mm} / \mathrm{s}$.

Figure 9.20 shows the top view of the single track predicted by the simulation. Two parallel lines are drawn considering the central steady-state region. The initial increase in the track width is less compared to $195 \mathrm{~W}$ and $800 \mathrm{~mm} / \mathrm{s}$. The end of the track is tapered with a sharp decrease in the width of the track. The behavior at the end is similar in both conduction and keyhole mode. The transverse melt profile is compared, and the results show the formation of the keyhole. 
(a)

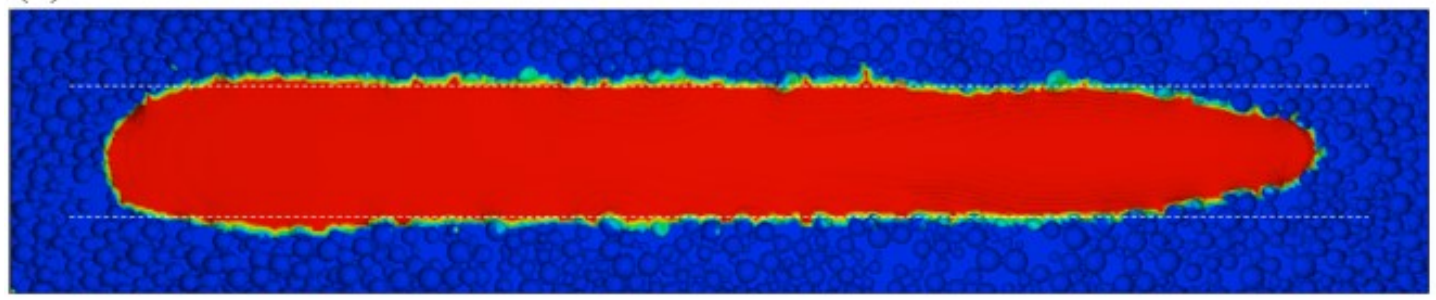

(b)
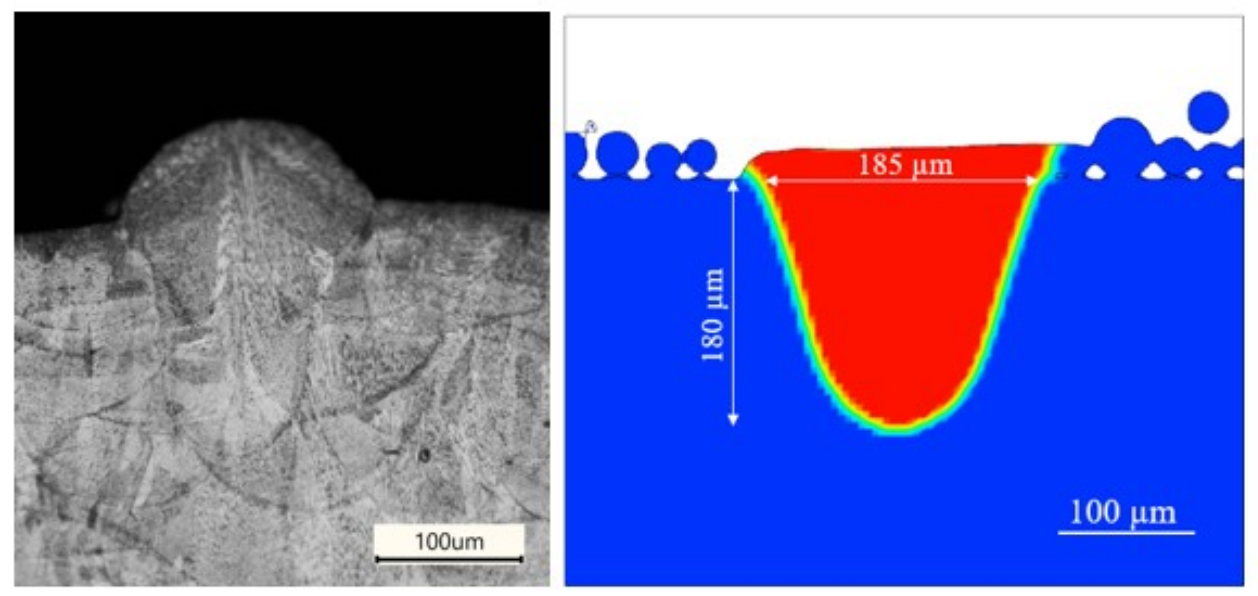

Figure 9.20 (a) Top view of the $2 \mathrm{~mm}$ single track formed with $195 \mathrm{~W}$ and $400 \mathrm{~mm} / \mathrm{s}$, and (b) Transverse melt pool obtained from the experiment and simulation.

\subsection{Conclusion}

A combined experimental and numerical study is performed to observe and understand the formation of transient and steady-state regions during the single-track scanning in the L-PBF process. The single tracks with scam lengths of $1 \mathrm{~mm}$ and $2 \mathrm{~mm}$ are fabricated using two scan speeds: $400 \mathrm{~mm} / \mathrm{s}$ and $800 \mathrm{~mm} / \mathrm{s}$. A white light interferometer is used to measure the surface morphology. Besides, metallography is performed to obtain the longitudinal melt profile and observe the variation in the depth of the melt pool along the laser scan direction. Finally, a powder scale numerical model is utilized to understand the formation of transient and steady-state regions. The results and analyses lead to the following conclusions. 
(1) The transient and steady-state regions are identified based on the track width. The track is wider at the initial transient region and has decreasing width at the final transient region. The steady region forms between the two transient regions and has a uniform width.

(2) The transient behavior did not have an obvious effect on the depth of the melt pool. However, there is a variation in the melt pool depth along the scan direction. Hence, the size of the melt pool measured from the transverse profile would depend on the location.

(3) The variation in the melt pool size is related to the difference in the thermal gradient in different regions. The numerical study showed that the thermal gradient is higher at the beginning of the scan. Hence initial bump with the wider track is formed as a result of the strong backflow of the melt pool and pronounced side flow.

(4) The width of the track becomes constant after the melt pool reaches a steady state. Besides, the melt pool width is lower at the laser application area; hence the end of the track has the tapered shape. 


\section{CHAPTER 10 RESIDUAL HEAT EFFECT ON THE MELT POOL GEOMETRY DURING THE LASER POWDER BED FUSION PROCESS}

\subsection{Introduction}

Laser powder bed fusion (LPBF) is an additive manufacturing (AM) process that uses a laser to melt the metallic powder particles layer by layer. Layer-wise manufacturing enables the fabrication of very complex geometries, which include lattice structures [128], topology optimized structures [129], etc. Such parts often consist of thin features formed by using few scan tracks. Besides, these thin features may be formed with very short scan lengths.

The parts are scanned with vectors parallel to each other in the LPBF process [58]. The smaller areas have shorter scan lengths, which result in high temperatures due to a little cooldown time. Yadroitsev and Smurov [130] studied the effect of hatch spacing on the surface morphology. The analysis of the cross-sections of the tracks fabricated on the build substrate shows the difference in the track height during the multitrack formation. The track at the beginning of the sequence is higher than the successive tracks. Besides, Pupo et. al [131] concluded that the surface morphology in a multitrack experiment is strongly affected by the hatch spacing due to heat accumulation at the melted zone. Moreover, heat accumulation during the multitrack scan affects the subsequent melt pool size [111]. Hu et. al [132] investigated the evolution of contact 
angle during multitrack scanning. The cross-sectional model showed that the contact angle decreased first and then became stable with increasing track number. The authors concluded that the trend is not affected by the changes in scan speed or hatch spacing, rather it is determined by the fluid flow driving forces and the lifetime of the melt pool. In this regard, numerical models are used to predict the fluid flow and melt pool size.

Lee and Zhang [25] utilized the powder scale numerical model to understand the effect of the first scan track on the melt pool of the second track. The melt pool shape for the first track is symmetric, while an asymmetric molten pool is obtained for the second track. This is because of the residual heat contained in the first track while depositing the second track. The preheating from the first track leads to the expansion of the second track towards the first track. The more symmetric melt pool can be obtained by increasing the hatch spacing, which results in the subsequent melt pool forming away from the heat-affected zone due to the melting of the previous track [133]. Criales et. al [134] performed the multi-track simulation for the LPBF process and observed the increase in the melt pool size in the subsequent tracks due to the heat-affected zones from the previous tracks. Bayat et. al [135] developed a numerical model to investigate the formation of the lack of fusion voids in Inconel 625 during multi-track/multi-layer LPBF. The study suggested that the lack of fusion pores, which are mostly elongated in the direction parallel to the scanning tracks, are mainly found in the lower layers de to the lower thermal energy compared to the higher levels. Gu et. al [136] studied the melt pool formation in multi-track, multi-layer, and multi-material during the LPBF process.

The difference in the surface height between the successive track is apparent in the LPBF process, and such variations in the surface morphology may lead to the internal 
porous structure during layer-wise fabrication [130]. Besides, the tracks formed have transient regions that would also affect the part quality, especially with the shorter scan vectors [84]. On the other hand, increasing the scan length may increase the residual heat which is also undesirable [137]. In this study, the effect of the residual heat on the quality of the melt pool during the small area fabrication is investigated. EOS M270 is used to fabricate the multi-track and multi-layer samples using Inconel 625. Three back and forth scan tracks using different levels of scan speed, hatch spacing, and scan lengths are formed. Besides, two layers are used to fabricate the multi-layer sample. The surface profile of the multi-track and multi-layer samples are obtained using the white light interferometer. The variation in the surface profile due to the back and forth scanning is analyzed. Further, the transverse metallography is performed at the laser turn around regions and the center of the tracks to observe the variation in the melt pool depth. In addition, a powder scale numerical model is developed to understand the effect of residual heat in the melt pool at the scan lengths of $0.5 \mathrm{~mm}$ and $1 \mathrm{~mm}$.

\subsection{Experimental Approach.}

Table 10.1 shows the experimental design used to form the multi-track and multilayer samples. Three levels of scan speed, two levels of hatch spacing, and three levels of scan lengths are used. Hence, multi-track and multi-layer samples with the combination of 18 parameters are fabricated for each. Three tracks are formed with back and forth scanning to investigate the effect of residual heat on the melt pool, while two layers are formed for the multi-layer study. 
Table 10.1 Experimental design used to fabricate multi-track samples.

\begin{tabular}{|l|l|}
\hline Laser Power $(\mathrm{W})$ & 195 \\
\hline Scan speed $(\mathrm{mm} / \mathrm{s})$ & $375,750,1500$ \\
\hline Hatch spacing $(\mu \mathrm{m})$ & 80,120 \\
\hline Scan length $(\mathrm{mm})$ & $0.5,1,1.5$ \\
\hline
\end{tabular}

Figure 10.1 illustrates the scanning pattern utilized in this study. Martin et. al [121] investigated the pore formation mechanism at the laser turn point during the LPBF process using in situ X-ray imaging and multi-physics simulation. The change in the laser scan velocity at the turn points leads to the formation of a deeper keyhole, and the pores are formed due to the collapse of the keyhole depressions. In this study, the acceleration and deceleration of the laser occur outside of the hatch lines, thus maintaining the constant energy input inside the scan lines. The laser jumps the distance of hatch spacing to form successive tracks, and the same pattern is used for the second layer, that is the first track in the second layer is formed on top of track 1 of the first layer.

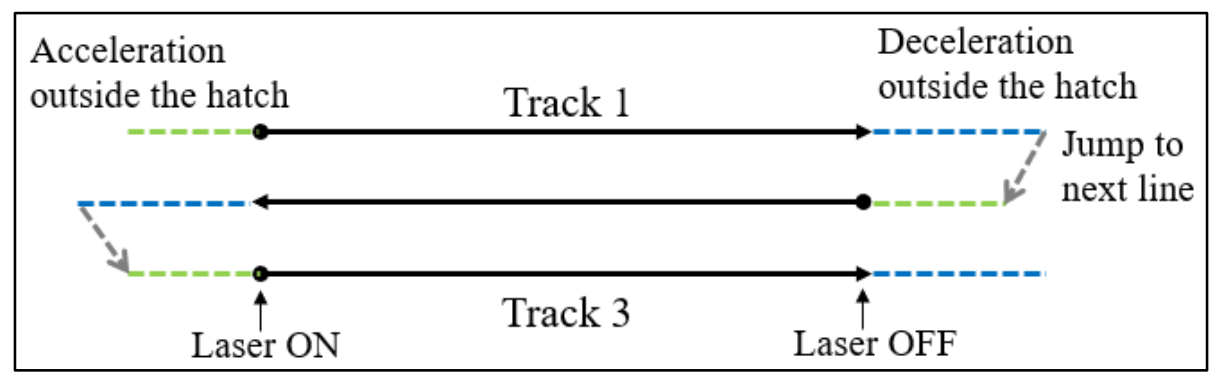

Figure 10.1 Schematic of the constant energy input in the scan lines during raster scanning. 
Figure 10.2(a) shows the sample design. EOS M270 is used to fabricate the multitrack and multi-layer samples with $40 \mu \mathrm{m}$ layer thickness, and the fabricated samples are shown in Figure 10.2(b). The semi-cylinder base is fabricated with EOS parameters, while the multi-track samples are fabricated on top of the base using user parameters listed in Table 10.1. The samples are detached from the build platform after the fabrication, and the support is removed by polishing.

(a)

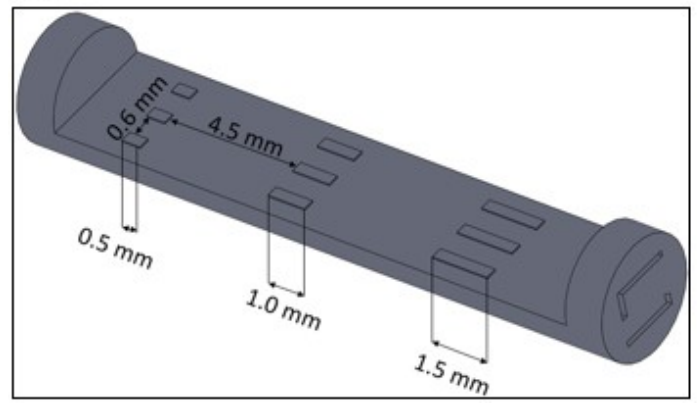

(b)

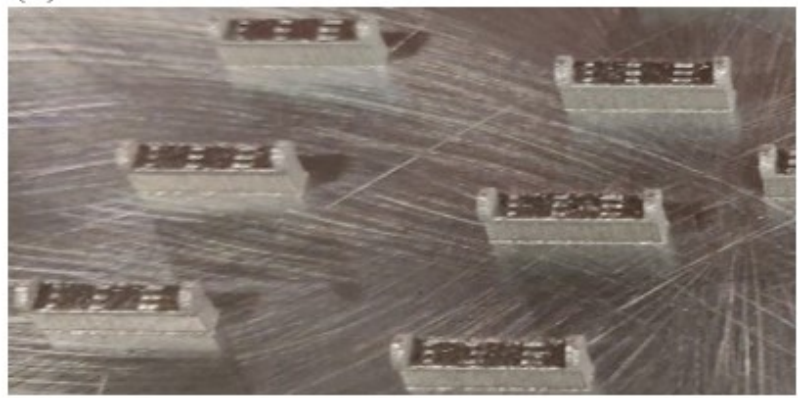

Figure 10.2 (a) Single layer designed on top of the semi-cylindrical base, and (b) Single layer samples fabricated using EOS M270.

A micro-CT scanner is utilized to observe the surface morphology and the internal pores. The pores may form due to keyhole melting or lack of fusion melting. However, the resolution of the micro-CT limited the measurement of the surface profile for detailed analysis. Hence, the surface morphologies of the test area are obtained using a white light interferometer. Besides, metallography is performed to determine the mode of melting and observe the variations in the transverse melt pool boundaries at different regions along the scan direction. 


\subsection{Numerical Approach}

\subsubsection{Discrete Element Method}

During the LPBF process, the build platform lowers by a layer thickness, and a layer of powder is spread using a roller or a recoater blade depending on the LPBF system. EOS M270 utilizes a recoater blade to spread the powder, and Figure 10.3 shows the schematic of major components involved in the layering process. It is important to model the powder spreading process to obtain a representative powder bed distribution. Hence, an opensource DEM code called LIGGGHTS is used to simulate the powder spreading process [16]. The powder particle generated in the dispenser is spread over the build platform by the recoater blade, as shown in Figure 4. The gap between the recoater and the build platform determines the powder distribution. However, the actual layer thickness of the powder may vary according to the build height, due to the inherent shrinkage during the building process. Some studies mention that the theoretical steadystate powder layer height is equivalent to the layer thickness divided by the packing density [17]. Assuming the powder packing density of $50 \%, 80 \mu \mathrm{m}$ powder is required to form a $40 \mu \mathrm{m}$ solid layer. As the single tracks are formed after reaching a steady state in the experiment, the gap distance of $80 \mu \mathrm{m}$ is used between the build platform and the recoater blade in the DEM simulation. However, the surface roughness of the substrate during the experiment is not considered in the simulation.

For the second layer DEM simulation, the surface formed after the first layer scanning is utilized. The powder bed from the first layer is lowered by $80 \mu \mathrm{m}$, which is the actual layer thickness of the first layer. However, the gap between the recoater blade and the base is reduced to $40 \mu \mathrm{m}$ for the second layer as the shrinkage is accounted for 
during the first layer scanning simulation. This method enables to investigate the variation in the powder distribution and layer thickness across the second layer due to the inherent surface roughness in the first layer. Figure 10.4 elaborates on the variation in the gap between the first layer and second layer powder spreading process.

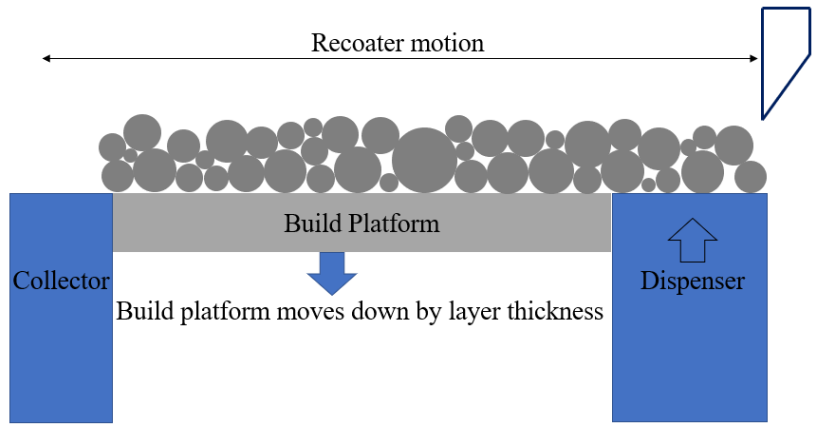

Figure 10.3 The working of the powder spreading process during LPBF process.
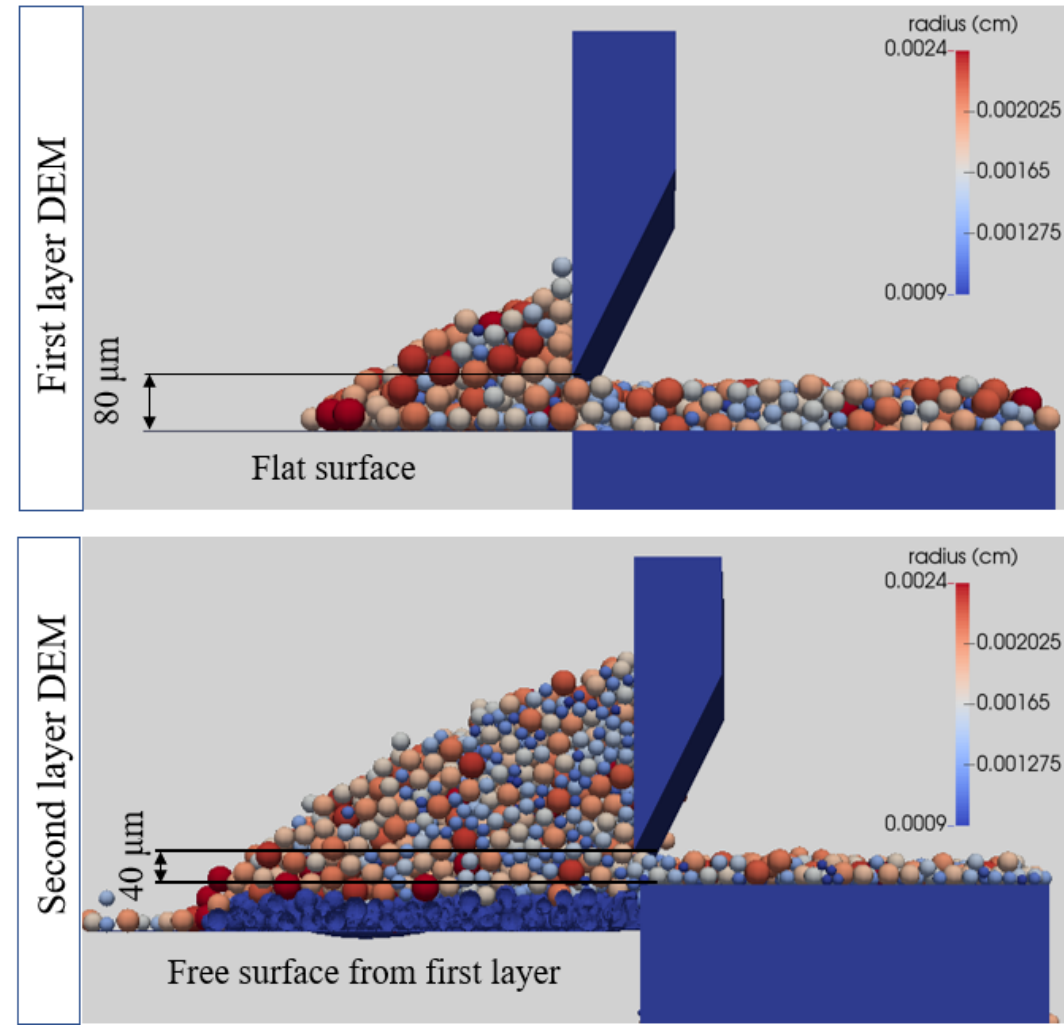

Figure 10.4 Consideration of actual powder height for first layer and second layer DEM simulation. 


\subsubsection{Thermo-fluid Simulation}

The layer of powder particle on top of the build platform is obtained from the DEM simulation, which is then imported to the FLOW-3D commercial software to perform the thermo-fluid simulation. Figure 10.5 shows the simulation domain used for a

$0.5 \mathrm{~mm}$ scan length. Two scan lengths are simulated, $0.5 \mathrm{~mm}$ and $1 \mathrm{~mm}$, and the length of the simulation domain is modified based on the scan length. Further, the simulation domain is divided into two zones: the inner zone has fine hexahedral mesh $(5 \mu \mathrm{m})$ where laser scanning is performed, and the outer zone has coarse mesh where the thermal gradient is low.

The temperature dependent IN625 material properties are assigned to the powder layer and solid substrate. Figure 10.6 shows the temperature dependent conductivity, specific heat capacity, and density of IN625. Besides, other material properties used in the simulation are listed in Table 10.2 [18]. A gaussian laser is modeled to perform the back and forth scanning and investigate the effect of scan length on the melt pool. After the completion of first layer scanning, the free surface is used to simulate the second layer powder spreading in LIGGGHTS. The obtained second layer powder is again imported to the thermo-fluid model, and finally back and forth scanning is performed.

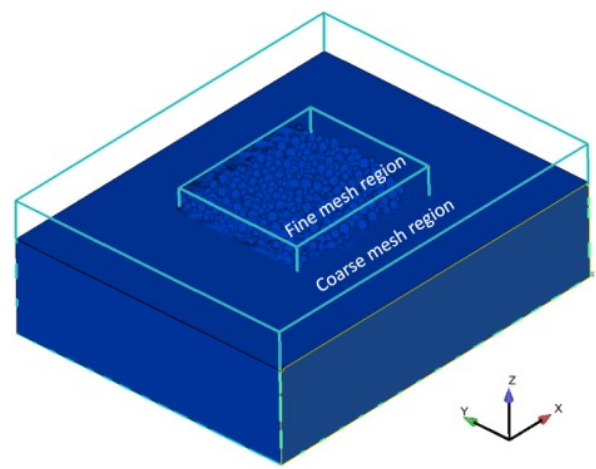

Figure 10.5 . Simulation domain used for $0.5 \mathrm{~mm}$ scan length simulation. 

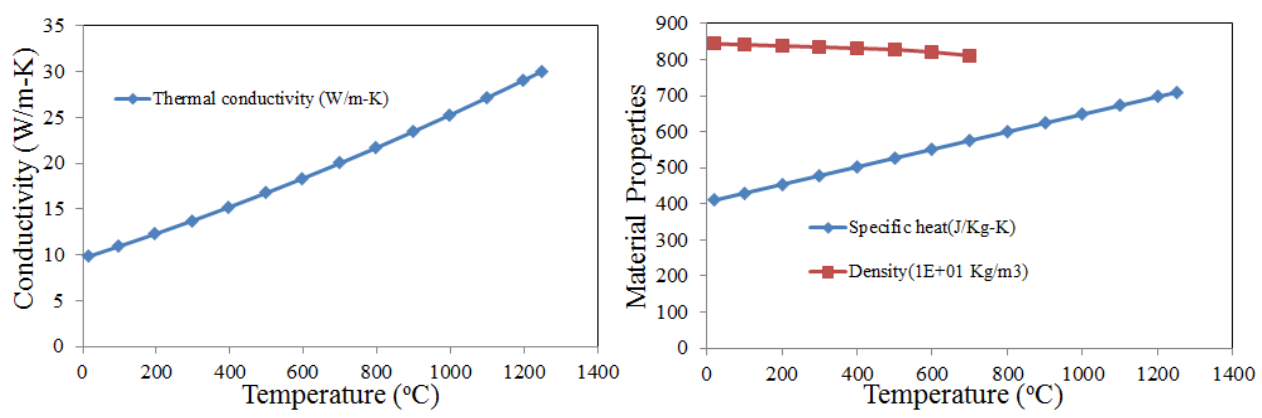

Figure 10.6 Temperature dependent material properties of Inconel 625 [19].

Table 10.2 Properties of In625 in a thermo-fluid simulation.

\begin{tabular}{ll}
\hline Parameters & Values \\
\hline Solidus temperature, $T_{S}(\mathrm{~K})$ & 1563 \\
Liquidus temperature, $T_{L}(\mathrm{~K})$ & 1623 \\
Boiling temperature, $\mathrm{T}_{\mathrm{v}}(\mathrm{K})$ & 3188 \\
Viscosity $(\mathrm{kg} / \mathrm{m}-\mathrm{s})$ & 0.007 \\
Surface tension $(\mathrm{N} / \mathrm{m})$ & 1.8 \\
Surface tension gradient $(\mathrm{N} / \mathrm{m}-\mathrm{K})$ & $-2 \mathrm{E}-05$ \\
\hline
\end{tabular}

10.4 Results and Discussion

\subsubsection{Experimental Results}

\section{Micro-CT Scanning}

The multi-track and multi-layer samples are scanned using Bruker micro-CT to observe the pores formed beneath the surface. Figure 10.7 shows the surface morphology of the multi-track and multi-layer samples fabricated with $195 \mathrm{~W}$ laser power, $120 \mu \mathrm{m}$ hatch spacing. Besides the detailed view of a $1 \mathrm{~mm}$ scan length is presented. The pores formed due to the parameter settings along with the free surface of the multi-track and multi-layer samples is observed. The keyhole pore formation with $195 \mathrm{~W}$ and $375 \mathrm{~mm} / \mathrm{s}$ is evident, while $750 \mathrm{~mm} / \mathrm{s}$ and $1500 \mathrm{~mm} / \mathrm{s}$ did not result in the keyhole porosity. The 
surface morphology is also affected by the process parameters. Although the scan speeds of $375 \mathrm{~mm} / \mathrm{s}$ and $750 \mathrm{~mm} / \mathrm{s}$ resulted in similar surface morphology, $1500 \mathrm{~mm} / \mathrm{s}$ resulted in very different surfaces. The higher speed resulted in lower track width, and the hatch spacing of $120 \mu \mathrm{m}$ forbade the proper overlap between the two tracks. Hence, the individual scan tracks are identified.

The pore number is higher for two-layer sample compared to the single layer as the possibility of pore formation increases with each scan track due to the formation of the keyhole. The surface morphology between the multi-track and multi-layer is very similar for the $375 \mathrm{~mm} / \mathrm{s}$ and $750 \mathrm{~mm} / \mathrm{s}$. This may be due to the use of the same scanning pattern for all layers. However, the surface worsened for $1500 \mathrm{~mm} / \mathrm{s}$ with increasing layer number. The higher hatch spacing at the lower energy density increased the surface roughness.

(a)

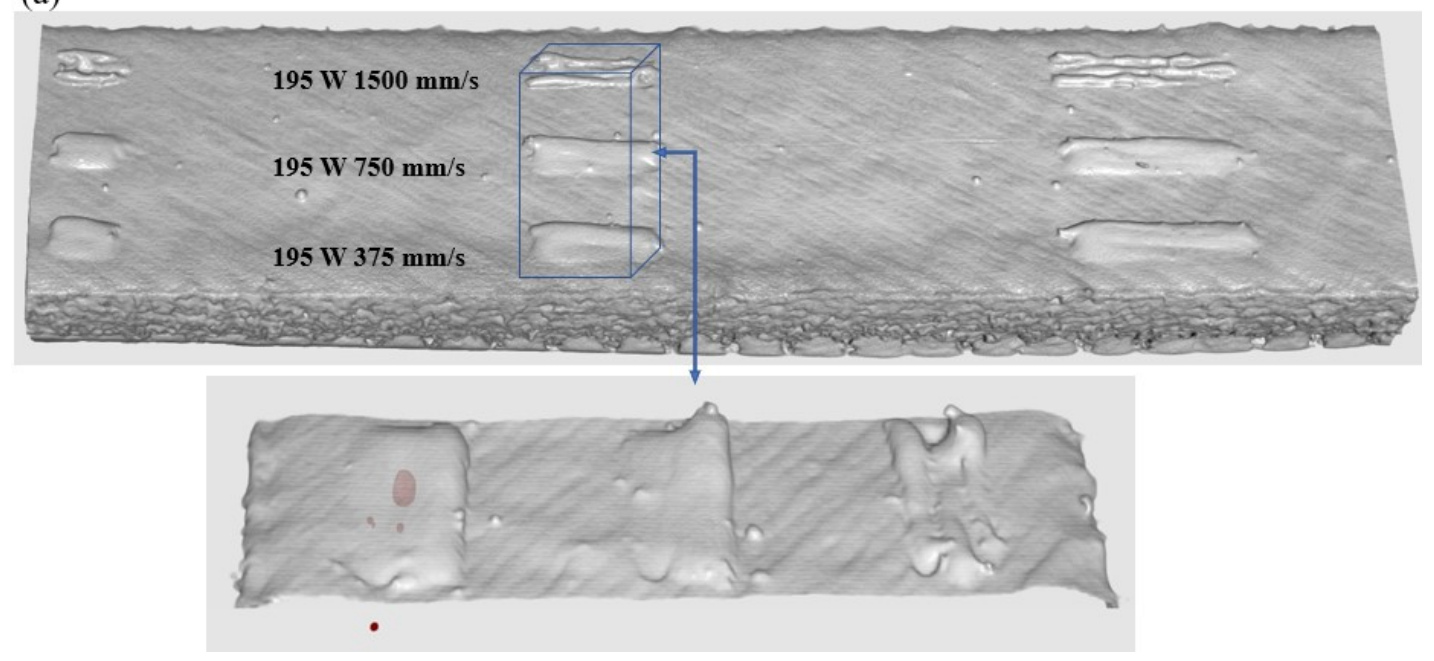


(b)

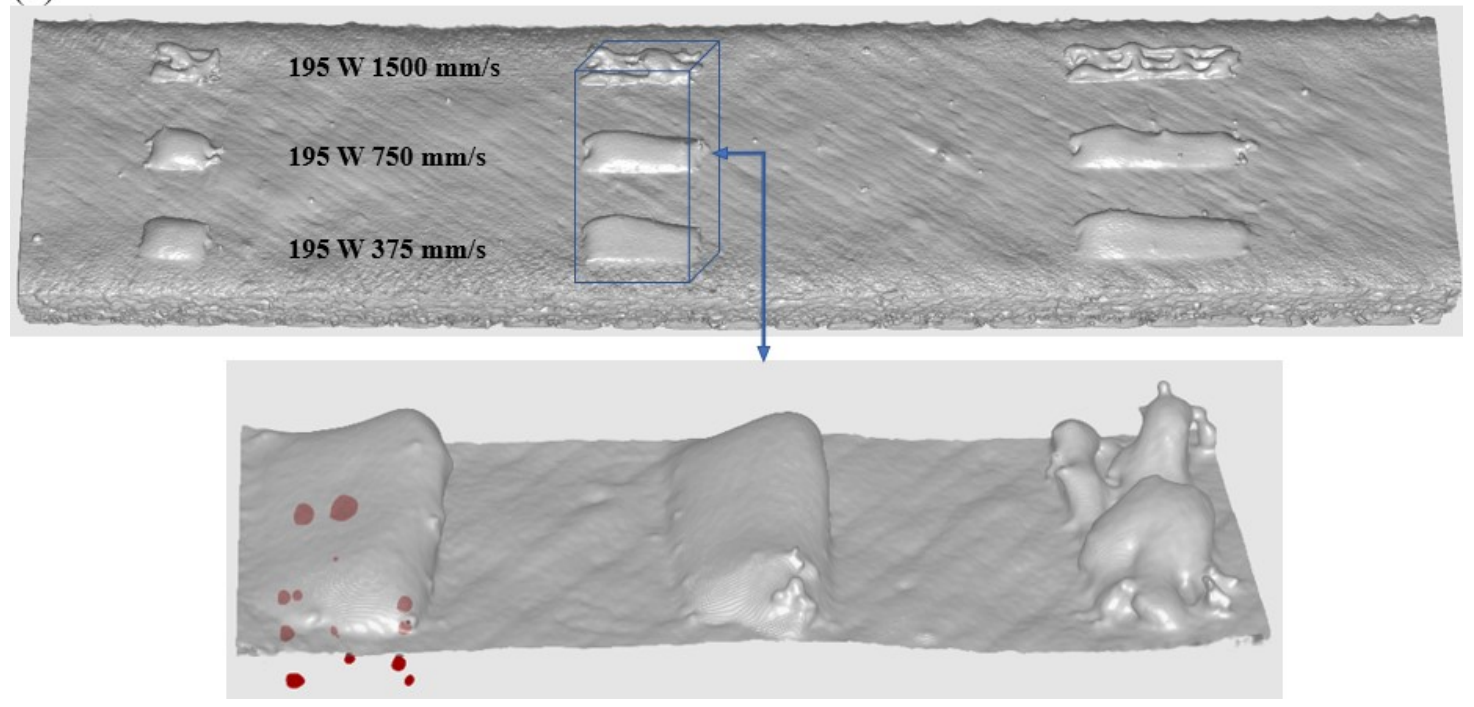

Figure 10.7 (a)Multi-tracks, and (b) Multi-layers formed with $120 \mu \mathrm{m}$ hatch spacing.

\section{$\underline{\text { Surface measurement }}$}

Figure 10.8 presents the surface morphologies of multi-track samples fabricated with $195 \mathrm{~W}$ laser power, $750 \mathrm{~mm} / \mathrm{s}$ scan speed and $120 \mu \mathrm{m}$ hatch spacing at different scan lengths. The effect of the residual heat is higher for $0.5 \mathrm{~mm}$ scan length as the three tracks resulted in a single bump. As the length was increased, the tracks became distinct, and the transient and steady region was more obvious with $1.5 \mathrm{~mm}$. Although the difference in the profile is evident, the maximum height difference between the track surface and the base is comparable, which is around $120 \mu \mathrm{m}$ for all scan lengths. Besides, the maximum track height is seen at the beginning of the track due to the formation of a hump. The hump is formed due to the backward surge of the melt pool at the laser turnon region [20]. Besides, the minimum surface height mostly lies at the end of the track. This is due to the formation of depression at the laser turn off region. At the laser turn regions, the melt pool formed during the scanning of the previous track may not fully 
solidify while another track is being scanned resulting to merge the two tracks. Hence, it is also noticed that the laser turning regions have continuity as if the laser itself turned to form an arc although the laser jumped between the tracks.

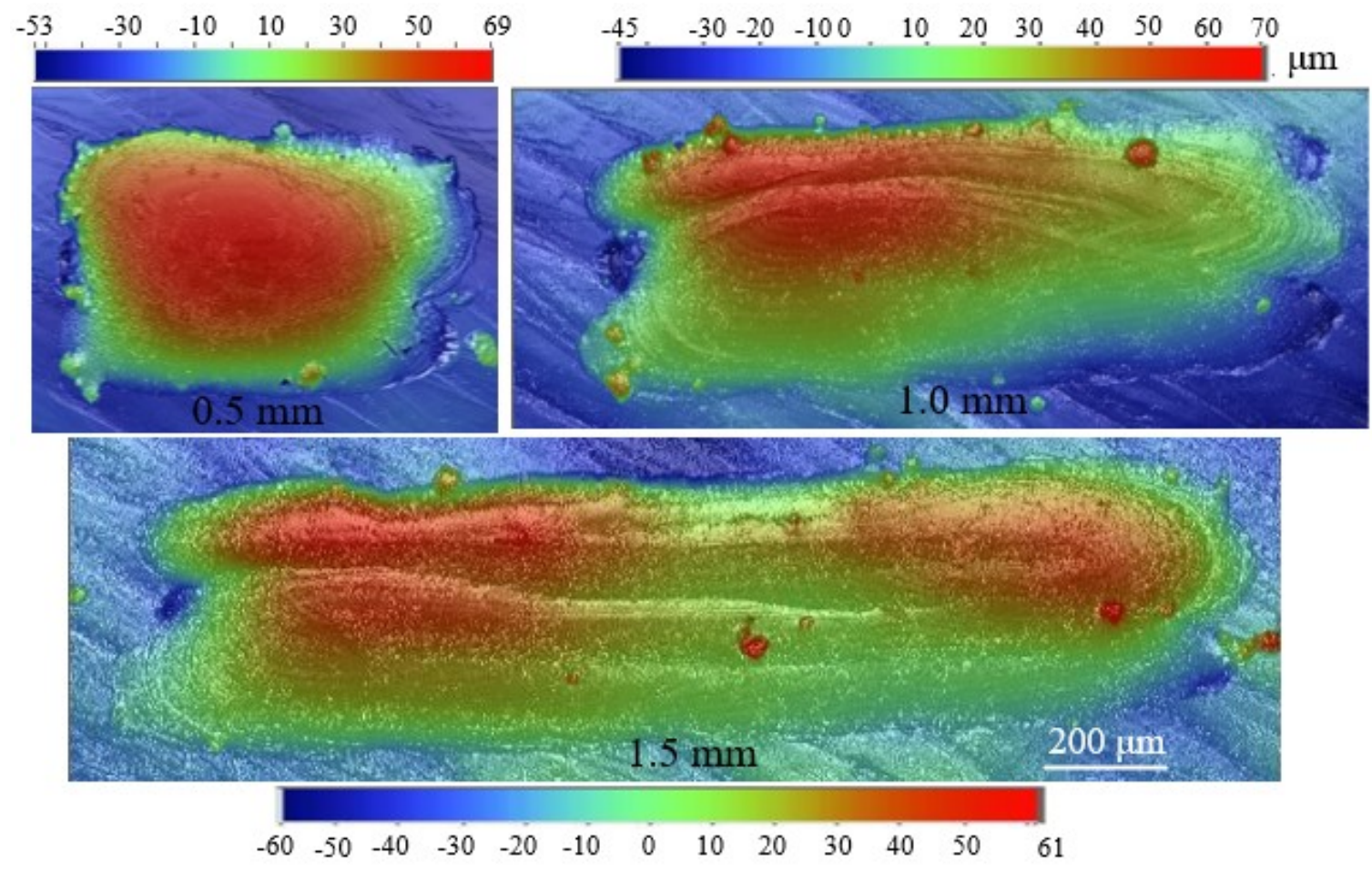

Figure 10.8 Multi-track profiles formed with $195 \mathrm{~W}, 750 \mathrm{~mm} / \mathrm{s}$ and $120 \mu \mathrm{m}$ at different scan lengths.

The surface formed with the same parameters is compared between multi-track and multi-layer, which is presented in Figure 10.9. The transverse profile is obtained to include the region with the maximum surface height as indicated in Figure 10.9. The surface height gradually decreased with the increasing number of tracks. The $2 \mathrm{D}$ profile shows the maximum surface height for the single-layer is around $75 \mu \mathrm{m}$, while the maximum height of the two-layer sample is $150 \mu \mathrm{m}$. The single-track experiments showed that the surrounding particles are depleted as the particles are entrained of 
powder particles in a shear flow of gas driven by a metal vapor jet at the melt track [21]. This denudation phenomenon increases the surface height of the first track significantly. On the other hand, the depletion of the powder particles in the surrounding would result in lower powder mass for the next track. Hence, the track height decreased for the second track.
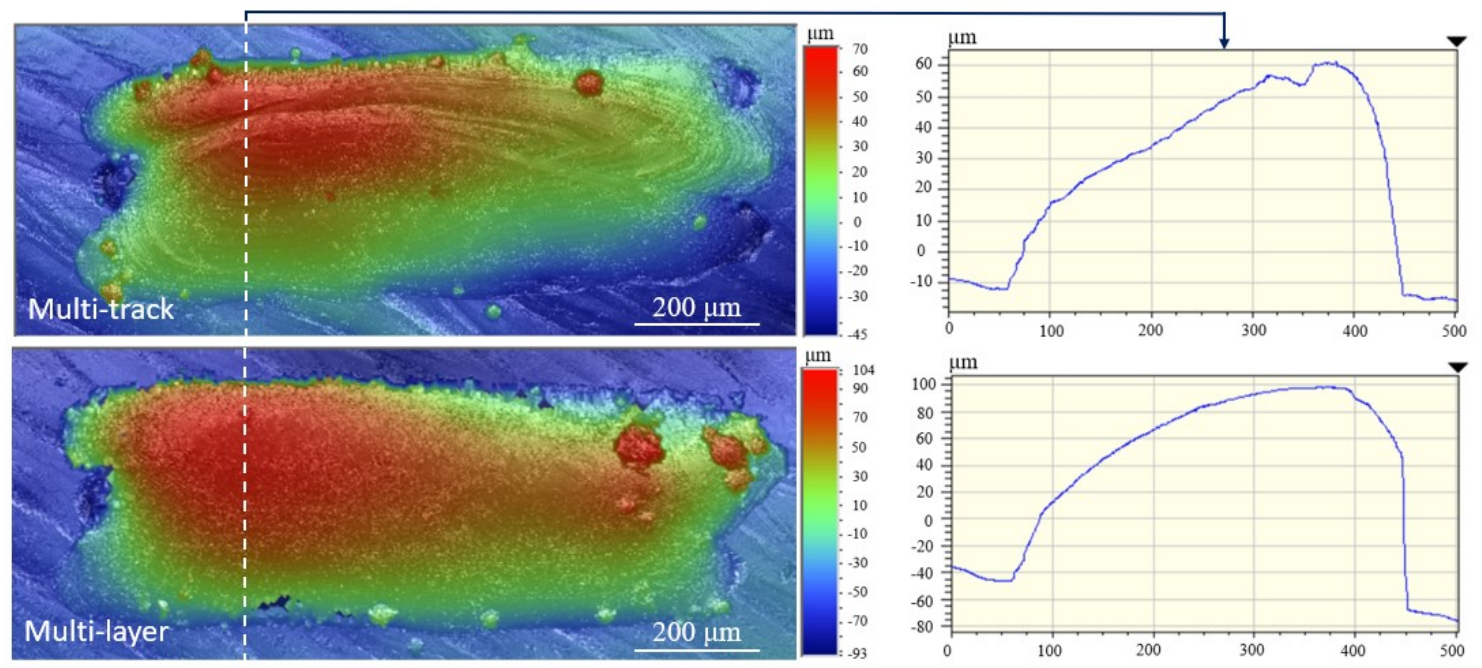

Figure 10.9 Surface morphology obtained with multi-track and multi-layer formed with $195 \mathrm{~W}, 750 \mathrm{~mm} / \mathrm{s}$ and $120 \mu \mathrm{m}$.

\section{Metallography}

The metallography is performed on the $0.5 \mathrm{~mm}$ and $1 \mathrm{~mm}$ long multi-track samples formed with parameters: $195 \mathrm{~W}$ and $120 \mu \mathrm{m}$ at different scan speeds. The melt pool boundaries at the start, middle, and end of the tracks as indicated in Figure 10.10(a) are obtained. Figure 10.10(b-d) shows the melt pool boundary obtained at three regions. The effect of the residual heat on the melt pool is apparent in this case. There is a significant overlap between the tracks for conduction mode $(750 \mathrm{~mm} / \mathrm{s})$ and keyhole mode $(375 \mathrm{~mm} / \mathrm{s})$ melting. However, there is no effect on the higher speed $(1500 \mathrm{~mm} / \mathrm{s})$ 
as the tracks do not overlap due to the mismatch between the track width and the hatch spacing. The overlap between track 1 and track 2 is maximum at slice 3 , where the laser turns from track 1 to track 2. Similarly, the overlap between track 2 and track 3 is maximum at slice 1 . The difference in the overlap between different regions is very small in this case.
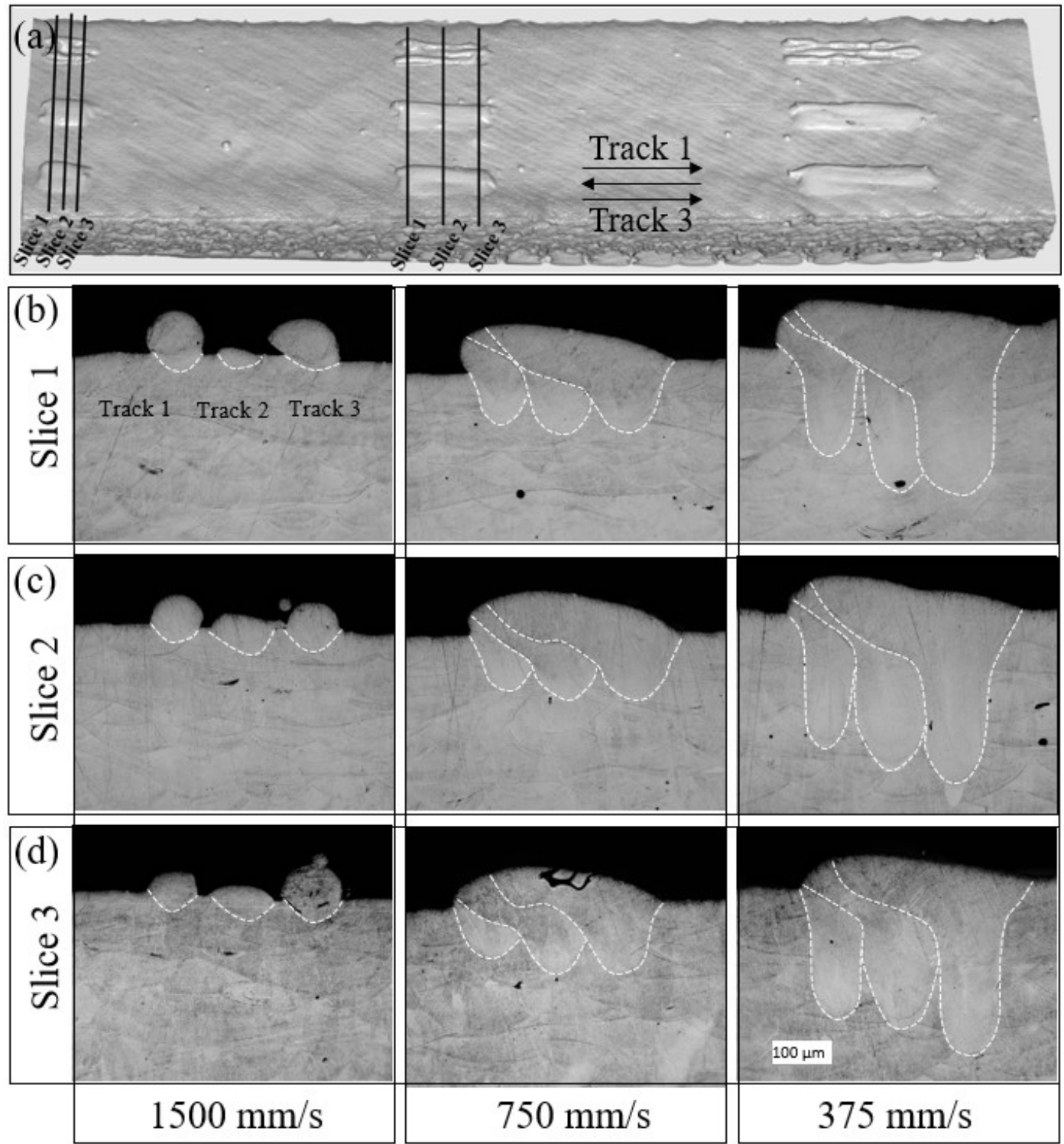

Figure 10.10 (a) Location of the three sections, and transverse melt pool boundary from (b) slice 1 , (c) slice 2, and (d) slice 3 of $0.5 \mathrm{~mm}$ scan length formed with $195 \mathrm{~W}$ laser power, $120 \mu \mathrm{m}$ hatch spacing and scanning speeds of $1500 \mathrm{~mm} / \mathrm{s}$ (left), $750 \mathrm{~mm} / \mathrm{s}$ (middle), an 
The melt pool boundary obtained from three different regions as indicated in Figure 10.10(a) is achieved for $1 \mathrm{~mm}$ scan length, which is shown in Figure 10.11. There is a noticeable difference between the melt pool boundary obtained with $0.5 \mathrm{~mm}$ and 1.0 $\mathrm{mm}$ scan lengths. The melt pool boundary obtained from slice 1 shows the overlap between track 2 and track 3, where the laser turns. At the laser turn region, the melt pool of the second track may not solidify completely when the laser scans the third track. Hence the melt pool from the third track would merge with the melt pool from the second track. However, there is no such overlap between track 1 and track 2, as the region includes the beginning of the first track and would solidify when the melt pool from the second track reaches the beginning of the first track. The melt profile obtained in the middle of the track (slice 2) shows a more consistent overlap between the tracks. This is different from overlaps noticed at slice 1, and the merging of the melt pool from two tracks is not evident in this region. The merging of the melt pools would depend on the lifetime of the melt pool. The melt profile is inclined towards the previous melt pool, which may be due to the residual heat from the previous track. The melt profile obtained at slice 3 shows the higher overlap between track 1 and track 2. Again, the higher overlap at the laser turn is due to the residual heat and unsolidified melt pool. The laser turns from laser 1 to laser 2 in this region which resulted in a larger overlap. Besides, the melt pol obtained from $1500 \mathrm{~mm} / \mathrm{s}$ shows three distinct tracks, with no or minor overlap between the tracks. This is due to the use of higher hatch spacing in relation to the track width resulted from using $1500 \mathrm{~mm} / \mathrm{s}$ at $195 \mathrm{~W}$ laser power. 


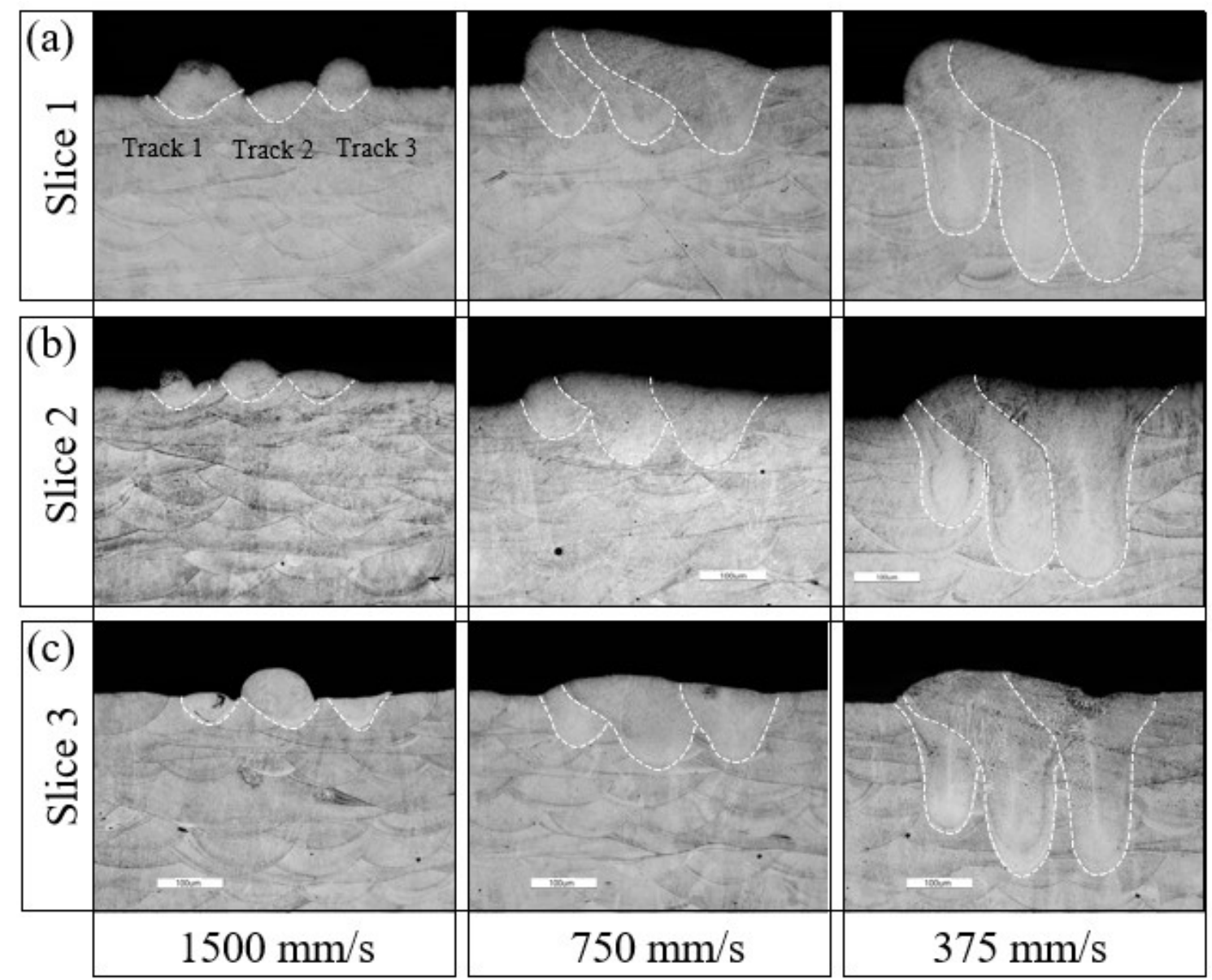

Figure 10.11 Transverse melt pool boundary of $1.0 \mathrm{~mm}$ scan length from (a) slice 1, (b) slice 2, and (c) slice 3 formed with $195 \mathrm{~W}$ laser power, $120 \mu \mathrm{m}$ hatch spacing and scanning speeds of $1500 \mathrm{~mm} / \mathrm{s}$ (left), $750 \mathrm{~mm} / \mathrm{s}$ (center), and $375 \mathrm{~mm} / \mathrm{s}$ (right).

The transverse melt pool boundary is also obtained from the two-layer sample. The multi-track experiment exhibited the existence of a huge variation in surface height along the longitudinal and transverse direction. This would cause the variation in the effective powder layer thickness for the second layer scanning, which in turn affects the laser penetration and the melt pool formation. Hence, the metallography is performed to detect the differences in the melt profile between the first layer and the second layer. Figure 10.12 shows the transverse melt pool boundary obtained from the $1 \mathrm{~mm}$ sample. 
The images correspond to the slice 1 location discussed in the multi-track metallography. The melt pool boundaries formed with the two layers are distinct, which shows the similarity in the melt pool between two layers due to the use of same scanning strategy. However, the surface profile of the first layer affected the melt pool depth, which is significant in the conduction mode melting (Figure (10.11(b)). Besides, lack of fusion and keyhole pores are observed for the incomplete melting parameter (Figure 10.11(a)), and keyhole parameter (Figure 10.11(c)) respectively. The lack of fusion pores is formed between the track, while the keyhole pore is formed inside the track.
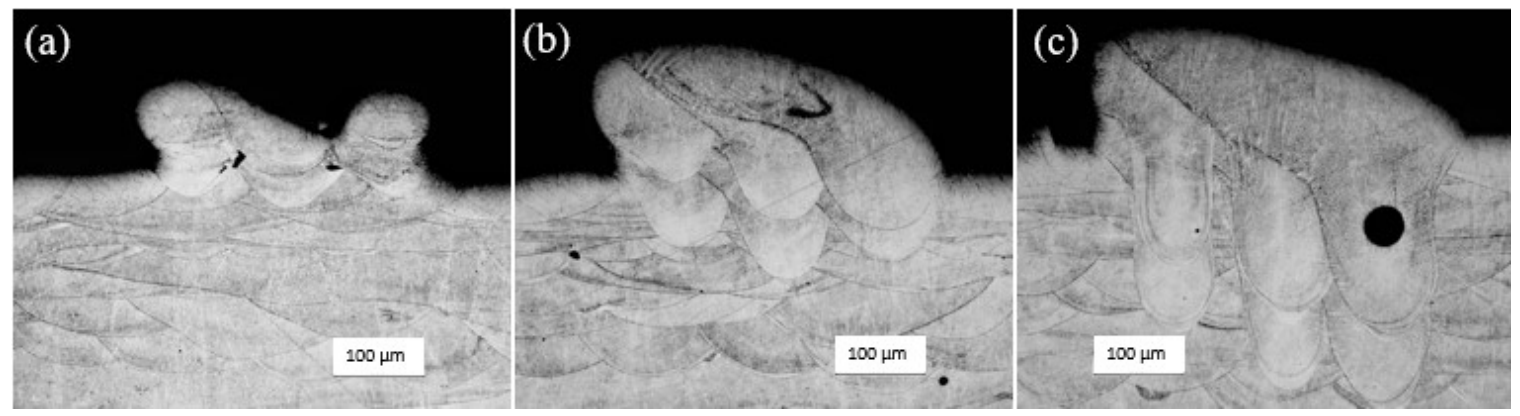

Figure 10.12 Micrographs of two-layers formed with $195 \mathrm{~W}$ laser power, $120 \mu \mathrm{m}$ hatch spacing, (a) $1500 \mathrm{~mm} / \mathrm{s}$ (b) $750 \mathrm{~mm} / \mathrm{s}$, and (c) $375 \mathrm{~mm} / \mathrm{s}$.

\subsubsection{Numerical Results}

The experimental result showed that the residual heat has considerable effect on the melt pool boundary and the surface formation. The residual heat may depend on the material property which affects the life of the melt pool. Hence, numerical simulations are performed to understand the effect of residual heat on the development of the melt pool. Besides, the variation in the actual powder layer thickness due to the variation in the surface height of the first layer is investigated. 


\section{$\underline{\text { Multi-track results }}$}

Figure 10.13 presents the temperature contour of the multi-track simulations performed with $0.5 \mathrm{~mm}$ and $1 \mathrm{~mm}$ scan lengths. The laser power, scan speed and hatch spacing used in the simulation are $195 \mathrm{~W}, 750 \mathrm{~mm} / \mathrm{s}$, and $120 \mu \mathrm{m}$, respectively. The length of the melt pool is around $1 \mathrm{~mm}$ with the used laser parameters. Hence, the effect of residual heat is significant in the case of $0.5 \mathrm{~mm}$. As the laser begins to scan the third track, the melt pool from the previous two tracks did not solidify in case of $0.5 \mathrm{~mm}$. However, the melt pool formed at the first track solidified when the laser started to scan the third track in case of $1 \mathrm{~mm}$ scan length. The lifetime of the melt pool would highly affect the microstructure formed during the L-PBF process.

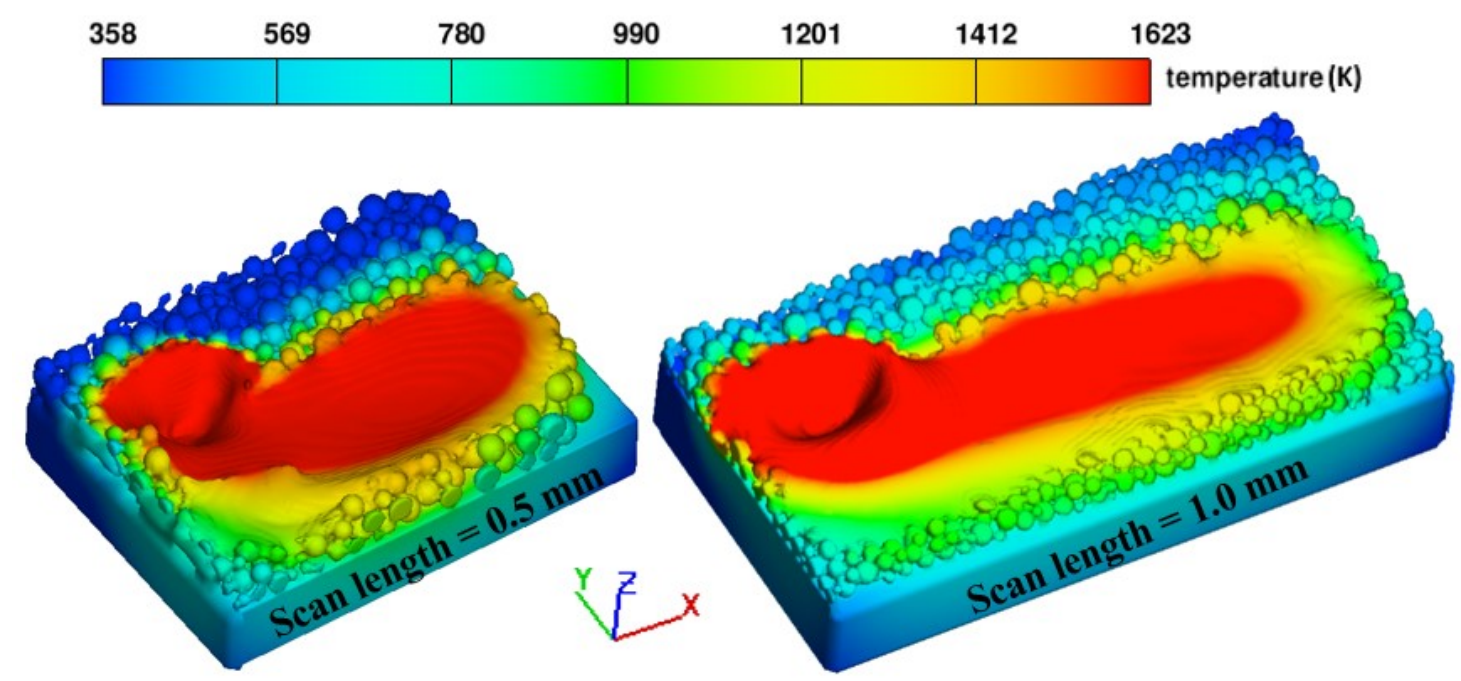

Figure 10.13 Temperature contour of the multi-track formed with $195 \mathrm{~W}$ laser power, $750 \mathrm{~mm} / \mathrm{s}$ scan speed, $120 \mu \mathrm{m}$ hatch spacing, and different scan lengths.

Figure 10.14 presents the transverse melt pool at different locations. Three slices discussed in the experiment (Figure 10.10) are analyzed for the simulation too. The melt pool obtained from the first track is indifferent at different locations, and the melt pool is 
mostly symmetrical. At the first location (slice 1), the melt pool formed with the second track is not affected by the first track as the temperature distribution shows that the melt pool has already solidified. However, the laser turns from track 2 to track 3 near location 1. Hence, the melt pool formed in track 3 scanning merged with the unsolidified melt pool from track 2. Similarly, the change in the shape of the melt pool between the successive tracks at the center of the tracks is obtained. As the laser reaches the center of the second and third tracks, the melt pool at the center of the previous tracks has already solidified. Hence, the melt pools do not overlap as that in the laser turn regions. Moreover, the shape of the melt pool is similar for the second and third track due to the similar effect of residual heat. Hence, it may be said that the melt pool is steadier along the transverse direction at the center of the $1 \mathrm{~mm}$ long back and forth scanning. The melt profile obtained at location 3 with laser turnaround from track 1 to track 2 is also presented. Again, the overlap between the tracks shows similar behavior at that of melt profile obtained at location 1 . As the laser scans the second track, the melt pool formed merges with the unsolidified melt pool from the first track. However, the third track is not affected by the residual heat. 


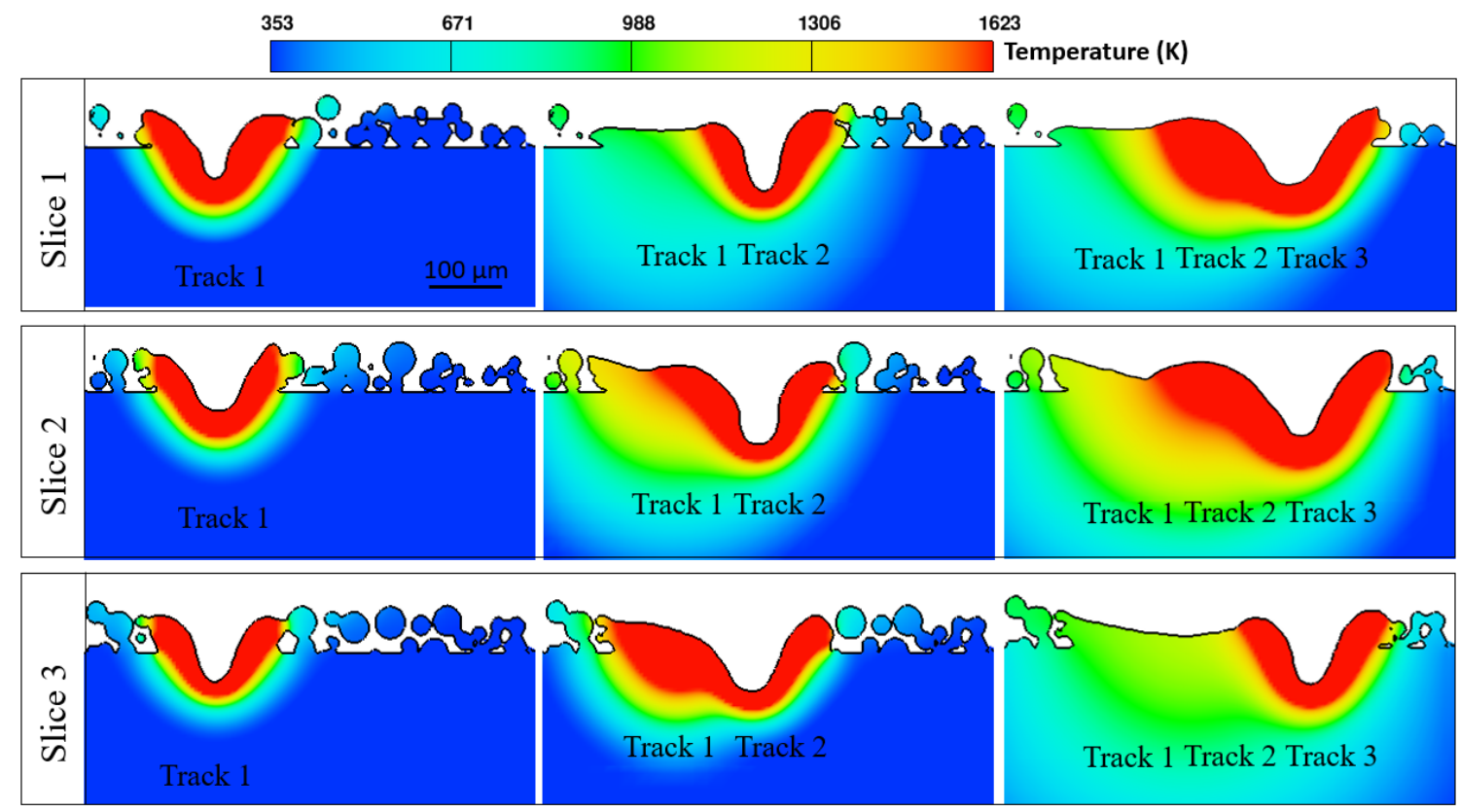

Figure 10.14 Temperature contour to observe the melt pool at different locations.

Figure 10.15 shows the top view of the three-tracks and the melt profile obtained from three locations. The transverse melt region from slice 1 and slice 3 show the difference in the melt pool boundary at the laser turning area, which is observed from the temperature profile shown in Figure 10.10. The melt pool overlap area is higher when there is laser turning as seen in the experiment. The melt pool taken at the center of the tracks shows the gradual increase in the melt pool depth from track 1 to track 3. 


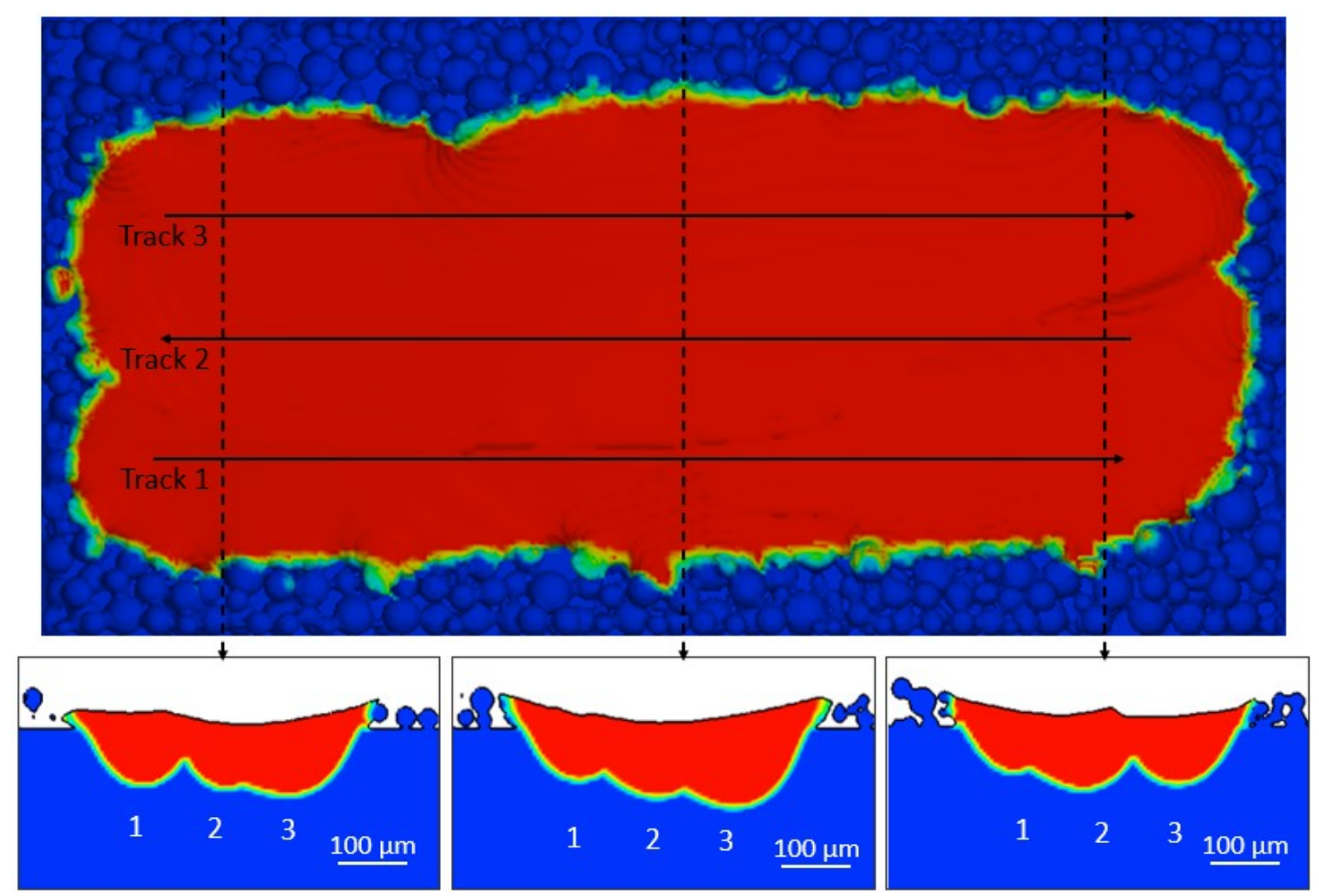

Figure 10.15 Melt profile formed at different region along the scan direction.

The analysis of the transverse melt pool revealed the effect of residual heat and the unsolidified melt pool on the melt pool boundary. Figure 10.16 presents the longitudinal view of the simulation performed with $195 \mathrm{~W}, 750 \mathrm{~mm} / \mathrm{s}$, and $80 \mu \mathrm{m}$ hatch spacing. The effect of scan length on the development of the melt pool and the melt pool depression is analyzed. There is a significant increase in the depression at the beginning of the second track due to the residual heat. Although the constant energy input is maintained, the residual heat from the previous track led to the formation of the deeper melt pool at the beginning of the second track. As the laser traveled to the middle of the track, the effect of residual heat lessened. However, the effect of residual heat is still present, and the depth of the melt pool increased. Again, the depth of the melt pool 
increased significantly as the laser changed direction for track 3 scanning. Besides, the transverse melt region taken at different regions from $0.5 \mathrm{~mm}$ and $1.0 \mathrm{~mm}$ cases show that the effect of residual heat at the laser turn region is similar. The major difference between the two cases is seen for the melt pool boundary at the center of the scan track, which is $0.25 \mathrm{~mm}$ for $0.5 \mathrm{~mm}$ scan length and $0.5 \mathrm{~mm}$ for $1.0 \mathrm{~mm}$ scan length. The depth of the melt pool increased significantly with an increasing number of tracks for $0.5 \mathrm{~mm}$, due to the residual heat. In contrast, the melt pool depth increased by a small measure for $1.0 \mathrm{~mm}$. In this regard, the effect of residual heat has a significant effect on the smaller tracks.

The effect of hatch spacing can be compared from Figure 10.15 and 10.16, which shows the transverse melt regions formed with $120 \mu \mathrm{m}$ and $80 \mu \mathrm{m}$ hatch spacings respectively for scan length of $1 \mathrm{~mm}$. The melt profiles obtained from three region shows that hatch spacing has a significant effect on the laser turn regions. There two melt pool tend to merge completely with lower hatch spacing.

(a)

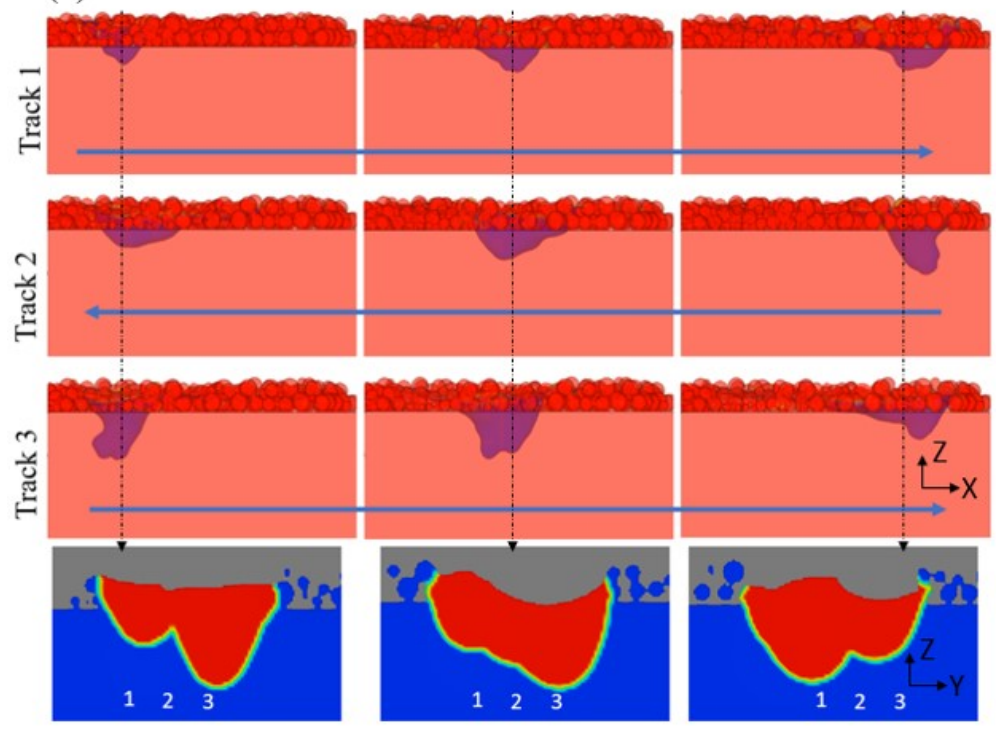


(b)

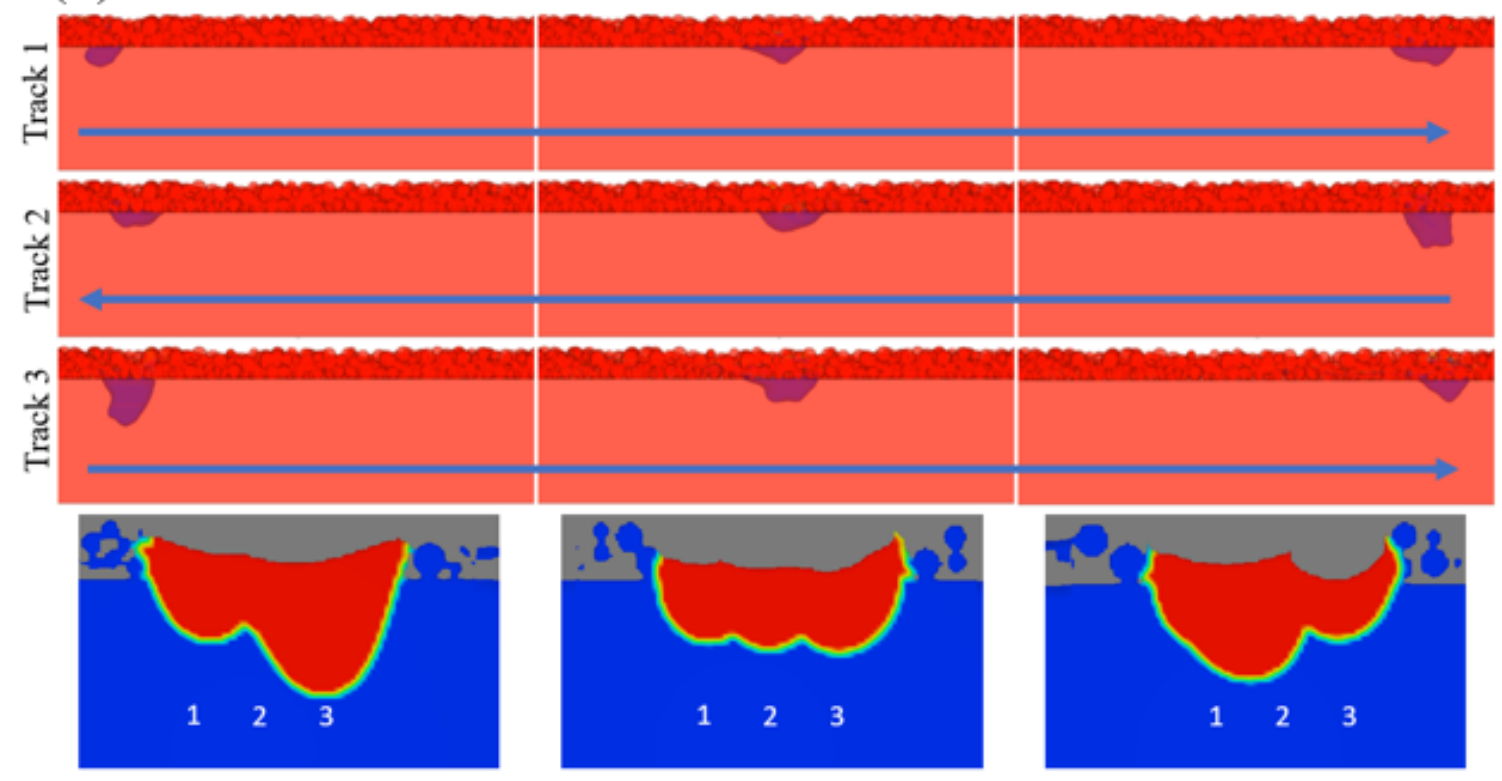

Figure 10.16 Evolution of melt pool and vapor depression with $195 \mathrm{~W}$ laser power, 750 $\mathrm{mm} / \mathrm{s}$ scan speed, $80 \mu \mathrm{m}$ hatch spacing, and scan lengths of (a) $0.5 \mathrm{~mm}$ and (b) $1.0 \mathrm{~mm}$.

\section{$\underline{\text { Multi-layer results }}$}

The multi-track simulation performed over the solid substrate showed the limitation of the mesh-based numerical model. The powder dynamics and its effect on the single-track morphology are not captured. However, the variation in the track surface due to the shrinkage is evident. The height of the track decreased from the start of the scan to the end, and from the first track to the third track. Consequently, Figure 10.17 shows the variation in the actual powder layer thickness obtained from the second layer DEM simulation. The green region is the mesh obtained from the first layer simulation, and the blue region is the powder particles being deposited over the first layer surface during the second layer DEM simulation. The resulting differences in the powder distribution would affect the subsequent melt pool formation. 


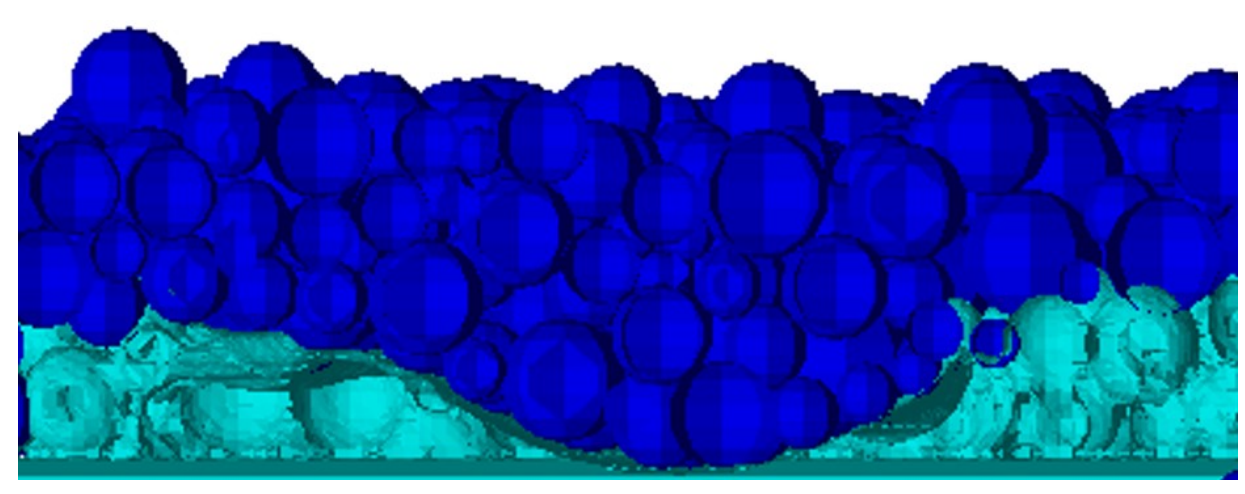

Figure 10.17 Variation in the actual powder layer thickness due to the variation in the surface height of the substrate layer.

Figure 10.18 presents the transverse melt profile obtained at three different locations from the two-layer simulation. The melt pool boundary of the first layer, powder distribution after the second layer DEM simulation, and the second layer melt pool boundary are compared at different locations. The actual powder layer thickness varies across the second layer as seen from the three slices taken at different locations. The powder distribution affected the second layer melt pool boundary. However, the trend of the melt pool boundary is like the first layer. There is a huge overlap between the tracks at the laser turn region. Besides, the second layer prediction would depend on the first layer surface morphology and the resultant powder distribution. As the simulation model predicted surface profile is contrasting to the experimental result, the resultant melt pool boundary is not comparable to the experimental melt pool boundary. 

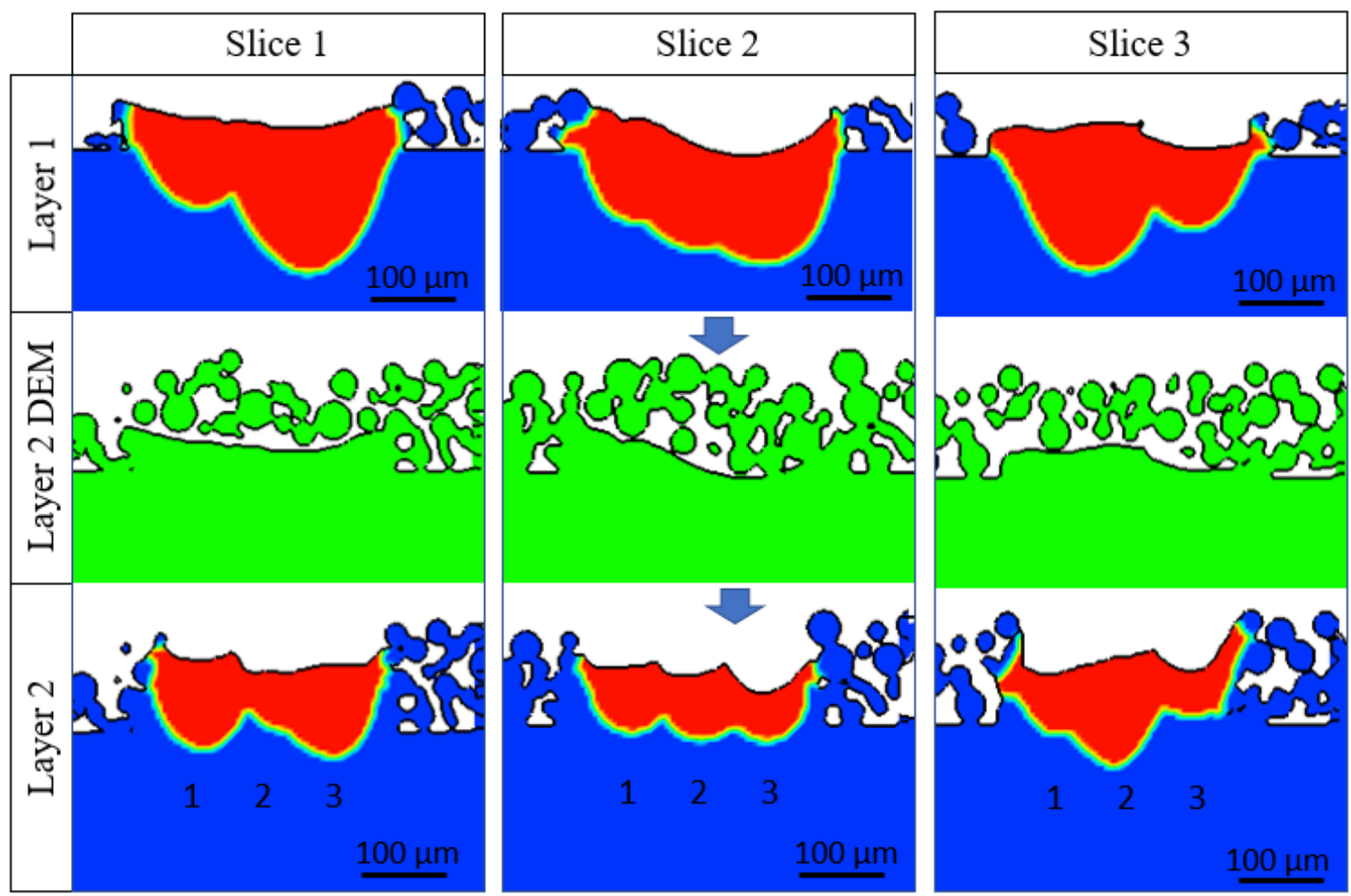

Figure 10.18 Second layer melt pool boundary obtained with $195 \mathrm{~W}$ laser power, 750 $\mathrm{mm} / \mathrm{s}$ scan speed, $80 \mu \mathrm{m}$ hatch spacing and $0.5 \mathrm{~mm}$ scan length.

The second layer simulation is also performed for low energy density case. Again, the transverse melt profile from three different regions is presented in Figure 10.19. The use of lower energy density shows the formation of the lack of fusion pores. The formation of a lack of fusion pore is apparent in the first layer simulation. In addition, the surface morphology worsened in the second layer. Such behavior is also observed in the experiment, as the surface became very distorted in the second layer scanning. 

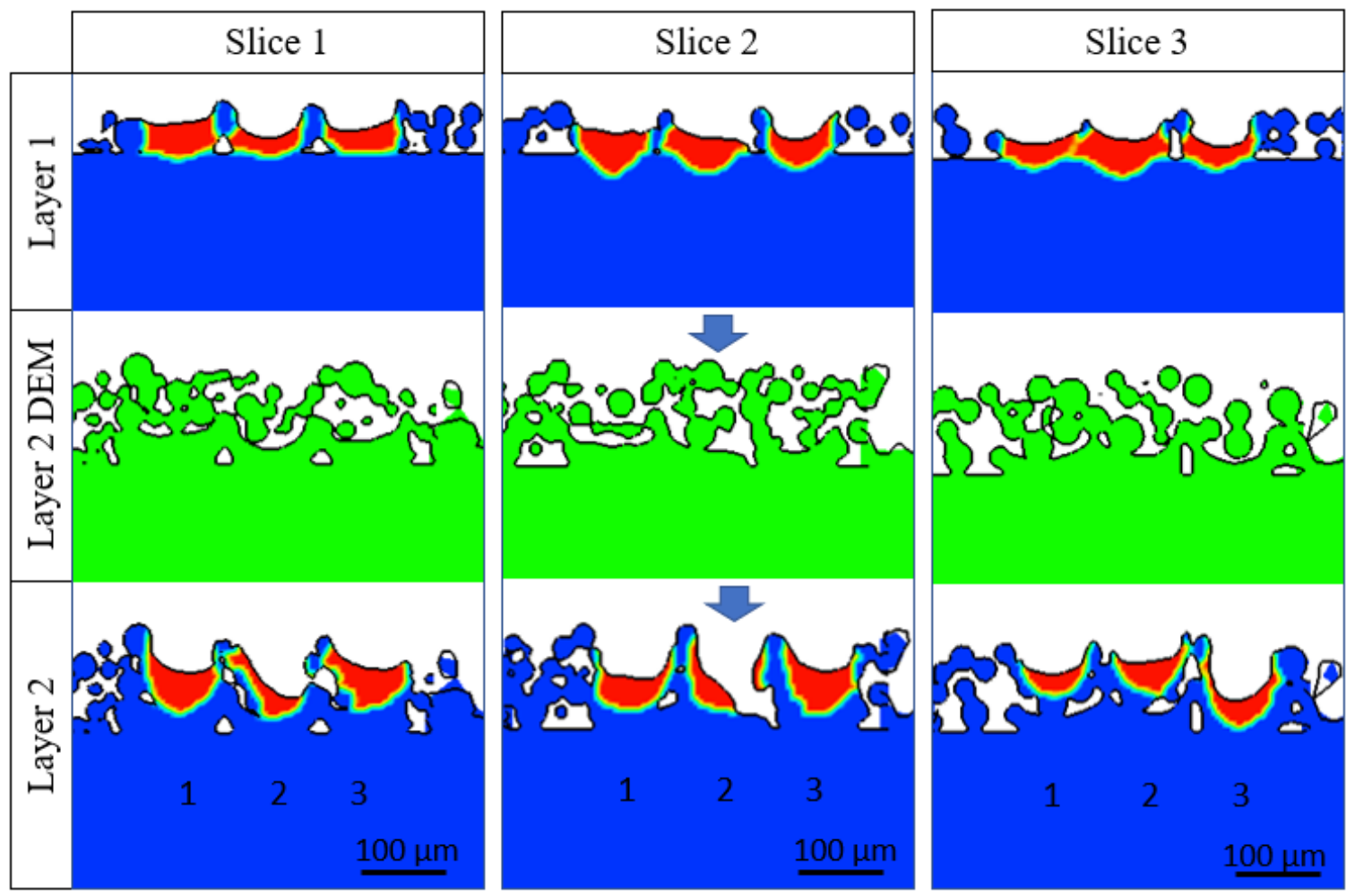

Figure 10.19 Second layer melt pool boundary obtained with $195 \mathrm{~W}$ laser power, 1500 $\mathrm{mm} / \mathrm{s}$ scan speed, $120 \mu \mathrm{m}$ hatch spacing and $0.5 \mathrm{~mm}$ scan length.

\subsection{Conclusions}

In this paper, a combined experimental and numerical study is performed to understand the effect of scan length on the residual heat and melt pool behavior during the multi-track and multi-layer fabrication. EOS M270 is used to fabricate IN625 multitrack samples with scan lengths of $0.5 \mathrm{~mm}, 1 \mathrm{~mm}$, and $1.5 \mathrm{~mm}$. A white light interferometer is used to measure the surface profile at first. Thereafter, the metallography is performed to reveal the transverse melt profile and observe the melt pool shape variations along the laser travel direction. A thermo-fluid numerical model is developed to understand the physics behind the discrepancies in the melt pool at different regions of the sample. The analyses of the results led to the following conclusions. 
- The residual heat effect is significant for shorter scan lengths, and the size of the melt pool increased significantly with increasing track number for a scan length of $0.5 \mathrm{~mm}$. Besides, most of the region remained in a molten state during the threetrack fabrication. Hence, the surface morphology of the $0.5 \mathrm{~mm}$ multi-track showed a single bump due to the surface tension.

- The transverse melt pool boundary obtained from the laser turn region showed a significant overlap between the tracks, both in experiment and simulation. The numerical analysis showed that the overlap is due to the merging of the melt pools from the two successive tracks. Besides, the overlap region is significantly higher for a $0.5 \mathrm{~mm}$ scan length compared to $1 \mathrm{~mm}$.

- The hatch spacing also has a significant effect on the formation of the subsequent melt pool boundary due to the presence of residual heat. Apart from the increase in the overlap, the depth of the melt pool increased significantly with lower hatch spacing.

- The surface profile of the first layer affected the actual powder layer thickness in the second layer, which in turn affected the second track formation in the second layer. Besides, the micrograph showed that the gap between the two-layer boundaries is higher for the region with a higher first layer surface height. 


\section{CHAPTER 11 BUILD LOCATION EFFECT ON THE SINGLE-TRACK PORE CHARACTERISTICS FORMED WITH SELECTIVE LASER MELTING PROCESS}

\subsection{Introduction}

The laser powder bed fusion (LPBF) process is a powder bed additive manufacturing (AM) process that utilizes a laser to melt the powder particles and solid substrate beneath. Hence, the laser parameters such as laser beam profile [138], laser power, and scan speed [106]would determine the mode of melting and quality of the scan track. Besides, the laser parameters, the powder size distributions [139], layer thickness [140], inert environment [141], etc. also introduce variability during the LPBF process. In addition, the layer-wise fabrication process leads to anisotropy, location-specific properties, residual stresses, distortion, etc. [142].

Wang [143] investigated the effect of spatial location on the tensile property during the LPBF of Hastelloy X alloy. The yield stress, ultimate stress, and elongation are found to be almost identical for the specimens build at the center and corners. Similarly, Zhang et. al [144] performed the analysis of variance and the results showed that the build location has less effect on the tensile property. Besides, Fitzgerald and Everhart studied the effect of the location on the structure and mechanical properties during the LPBF of 316L stainless steel [145]. The data showed that spacing between the 
parts in the build influenced the tensile properties if the spacing were large enough, while the build height did not have much effect on the tensile properties.

Ferrar et. al [141] studied the repeatability of the LPBF process within a build and between different builds and concluded that the build location affected the porosity and the compression strength. The location-dependent properties within and between the builds in the LPBF process is due to the differences in the microstructure, residual stress, defects, etc. [146] Besides, Edwards and Ramulu [32] noted the importance of porosity characterization with respect to the build location and orientation. The presence of location-specific property in the LPBF process impedes the part qualification desired for different applications. Hence, the parameters which introduce or enhance such effect need to be investigated to obtain better control of the parts over the build volume. In this study, the single tracks are fabricated at the extreme locations to investigate the effect of the spatial placement of the tracks on the porosity. Four single tracks are formed with 195 W laser power and four scan speeds $(200 \mathrm{~mm} / \mathrm{s}, 400 \mathrm{~mm} / \mathrm{s}, 600 \mathrm{~mm} / \mathrm{s}$, and $800 \mathrm{~mm} / \mathrm{s})$ inside the hollow cylinder samples, which is fabricated at the center and four corners of the build platform. Among the four single tracks, $200 \mathrm{~mm} / \mathrm{s}$ and $400 \mathrm{~mm} / \mathrm{s}$ results in keyhole melting while $600 \mathrm{~mm} / \mathrm{s}$ and $800 \mathrm{~mm} / \mathrm{s}$ results in conduction melting. The pores formed due to the laser parameters are investigated using a micro-CT scanner.

\subsection{Experiment Design and Sample Fabrication}

In this study, four scanning speeds $(200 \mathrm{~mm} / \mathrm{s}, 400 \mathrm{~mm} / \mathrm{s}, 600 \mathrm{~mm} / \mathrm{s}$, and 800 $\mathrm{mm} / \mathrm{s}$ ) are used with constant laser power of $195 \mathrm{~W}$ to investigate the effect of build 
height and location on the porosity. The hollow cylinder design [106] is utilized (Figure 11.1(a)), and four tracks are accommodated in one sample ( Figure 11.1(b)). The single tracks are formed with the user parameters, while the cylinder is formed with default Ti6Al-4V EOS parameters $(170 \mathrm{~W}$ laser power, $1250 \mathrm{~mm} / \mathrm{s}$ scan speed, and $100 \mu \mathrm{m}$ hatch spacing). The sample is built in the Z-direction, and the hollow region of the cylinder is filled with the powder particles after the build due to the nature of the process.

The spatial distribution of the samples is shown in Figure 11.1(c). The cylinder samples are rotated by 45 degrees to minimize the interaction between the part and the recoater blade. One sample is fabricated at the center (C) and four samples are fabricated at the corners (C1-C4) of the $250 \mathrm{~mm} \times 250 \mathrm{~mm}$ build plate. Besides, three samples are fabricated at different build heights. Although the build height is different, the single tracks are formed at the same focal distance. The distance in height between the successive single track is $49.98 \mathrm{~mm}$, which corresponds to 1666 layers since $30 \mu \mathrm{m}$ layer thickness is used. Figure 11.1(d) shows the samples fabricated at different locations and different heights. The samples are fabricated using the EOS M270 system, which utilizes $200 \mathrm{~W}$ Ytterbium $(\mathrm{Yb})$ fiber laser. The laser motion is controlled by the galvo mirrors and focused on the build platform using the F-theta lens. The system is capable of variable focal diameter from $100-500 \mu \mathrm{m}$, while the constant beam diameter of $100 \mu \mathrm{m}$ is used during the fabrication of Ti-6Al-4V. The powder size distribution of $0-45 \mu \mathrm{m}$ is used. 

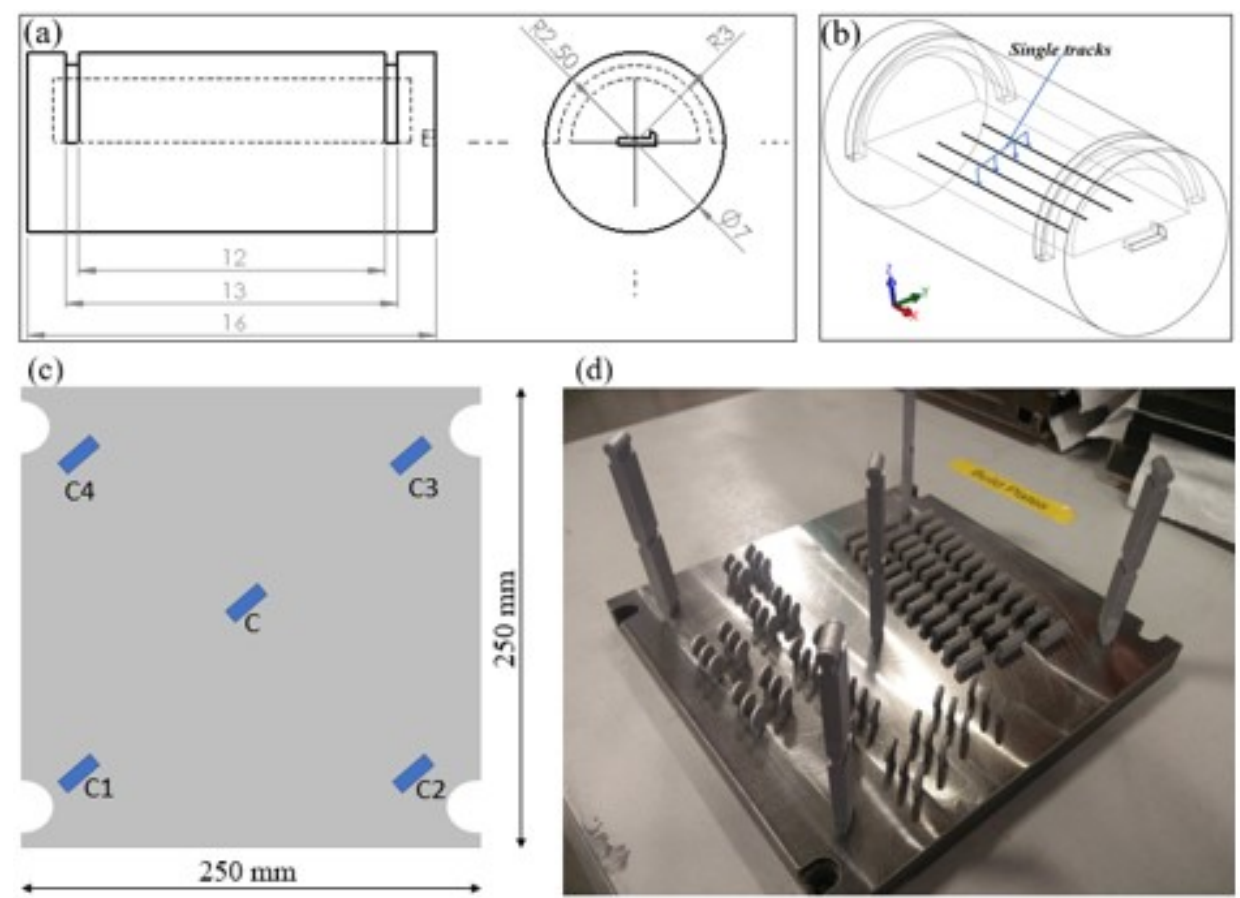

(d)

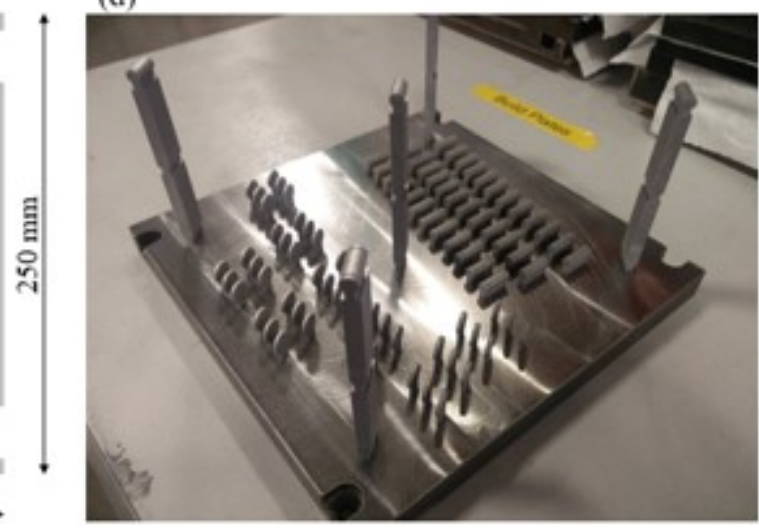

Figure 11.1 (a) CAD model of the specimen (unit in $\mathrm{mm}$ ), (b) Single tracks designed on top of previously deposited semi-cylinder, (c) Spatial distribution of the samples, and (d) Samples fabricated at different locations and different build heights.

\subsection{Results and Discussion}

The CT results obtained after the image reconstruction is analyzed. Figure 2 shows the longitudinal cross-section of the single tracks formed with $195 \mathrm{~W}$ and 200 $\mathrm{mm} / \mathrm{s}$ at different locations and the same build height of $106.56 \mathrm{~mm}$. The distribution of the pores along the laser travel direction is compared. The density of the pores is lower for locations $\mathrm{C}$ and $\mathrm{C} 1$, while location $\mathrm{C} 3$ had visibly more pores than other locations. The effect of the location on the pore number and distribution is evident from Figure 11.2. However, there may be other parameters that may induce variability in the keyhole porosity besides the location. The instabilities of the keyhole that leads to the pore formation is a complex interaction and would have variation for the same material and 
same process conditions. Besides, the EOS M270 system has an undirected inert gas flow, which may also contribute to the variability in the keyhole pores [147].

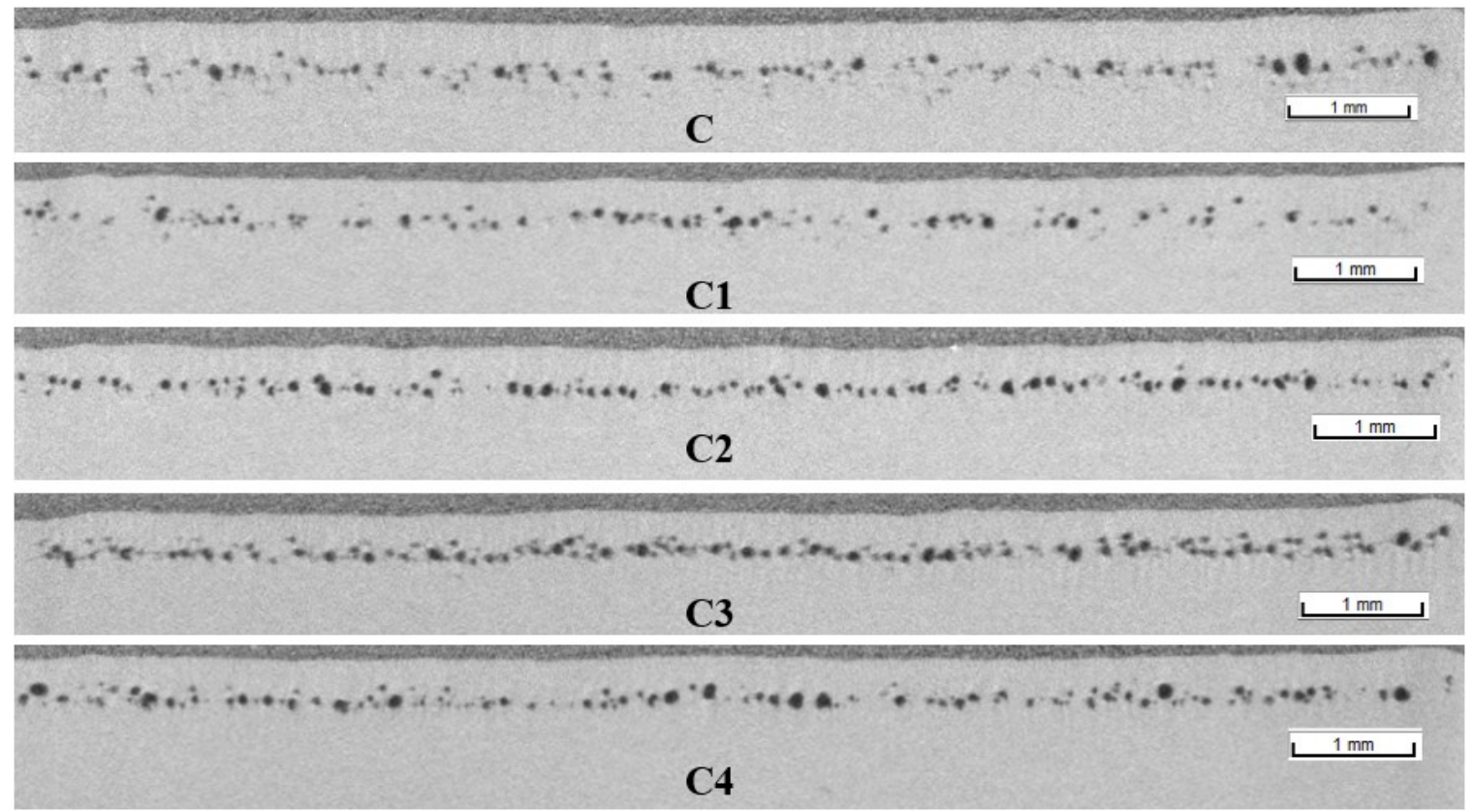

Figure 11.2 2D slices of $200 \mathrm{~mm} / \mathrm{s}$ formed at different locations (build height $=106.56$ $\mathrm{mm})$.

The average pore number and volume obtained from the keyhole parameters, that is $195 \mathrm{~W}, 200 \mathrm{~mm} / \mathrm{s}$ and $195 \mathrm{~W}, 400 \mathrm{~mm} / \mathrm{s}$, are summarized in Figure 11.3(a, b). The results show that the location has a higher effect on porosity compared to the build height. Besides, the porosity is highest at a corner located away from the build chamber window and towards the dispenser platform (C3). The average diameter and depth of the pores are also affected by the location. The average pore diameter is maximum for the single tracks $(195 \mathrm{~W}, 200 \mathrm{~mm} / \mathrm{s}$, and $195 \mathrm{~W}, 400 \mathrm{~mm} / \mathrm{s})$ formed at location C3. The higher average pore diameter at this location resulted in a significant increase in the pore volume, while there is no significant increase in the pore number. In contrast to the pore 
number and diameter, the average depth of the pores is minimum at location $\mathrm{C} 3$. Besides, the trend of the diameter and depth follows for both keyhole melting cases, with the pore depth decreasing when there is an increase in pore diameter. It is also observed that the variability due to the build height is lower compared to the variability due to the location in the case of $195 \mathrm{~W}$ and $200 \mathrm{~mm} / \mathrm{s}$ case. Ferrar et. al [141] showed that the variation in the porosity over the build platform is higher in the absence of the directed gas flow inside the build chamber. Hence, the variation in the porosity at a different location may be due to the undirected inert gas flow inside the build chamber in EOS M270 [141, 148].

(a)

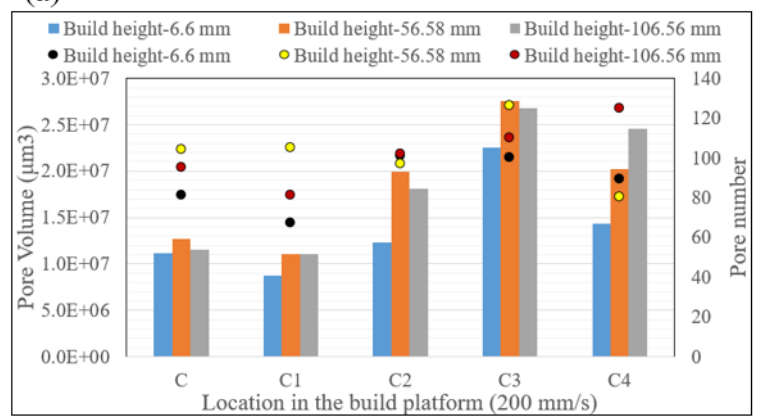

(c)

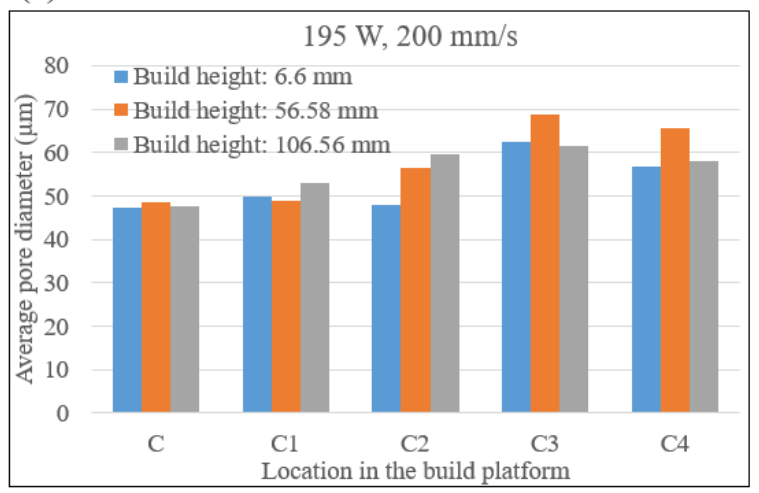

(b)

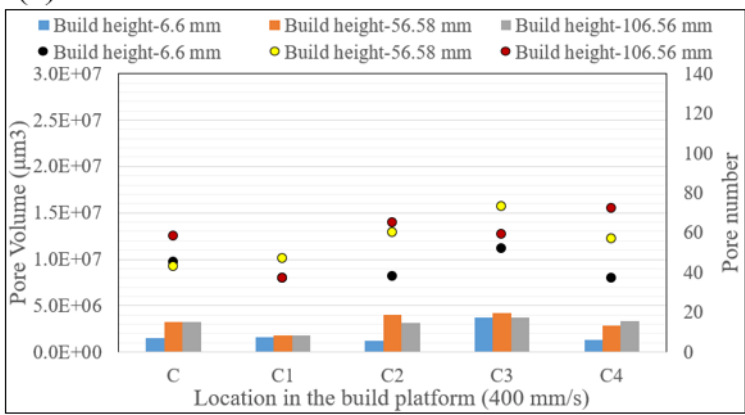

(d)

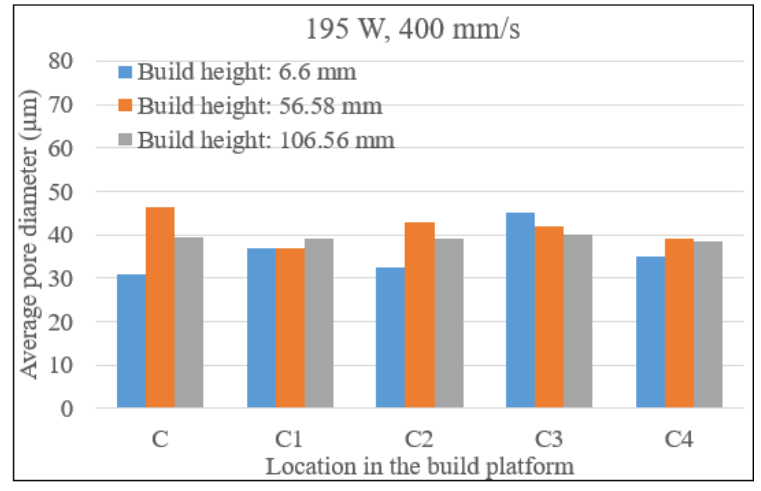


(e)

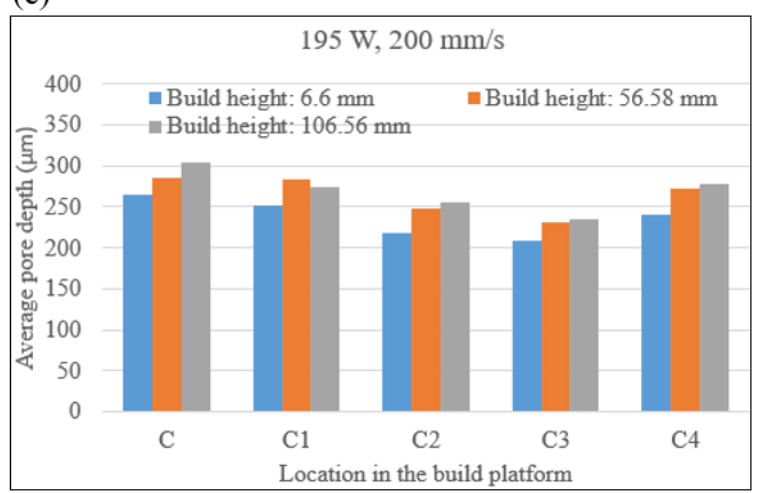

(f)

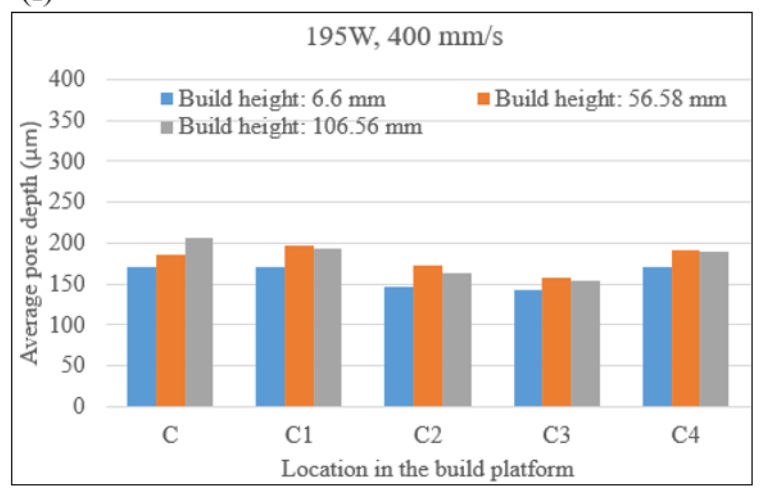

Figure 11.3 Characteristics of pores formed with $195 \mathrm{~W}, 200 \mathrm{~mm} / \mathrm{s}$ and $195 \mathrm{~W}, 400 \mathrm{~mm} / \mathrm{s}$ (a), (b) pore number and volume, (c), (d) Average pore diameter, and (e), (f) average pore depth.

The $2 \mathrm{D}$ pores from micrographs are compared with the $2 \mathrm{D}$ pores obtained with micro-CT in Figure 11.4. The series of pores are comparable; however, the size of the pores appear smaller for micro-CT compared the metallography. Both micro-CT and micrographs measurements are influenced by several factors. CT results are influenced by image artifacts such as misalignment, X-ray beam hardening, ring artifacts, etc. On the other hand, micrographs can be influenced by light and focus settings and stitching operations [110]. Besides, pores with sizes lower than the resolution of the micro-CT cannot be detected. Hence, the small pores observed with the metallography are not visible from the $\mathrm{CT}$ results. 
(a) $200 \mathrm{~mm} / \mathrm{s}$
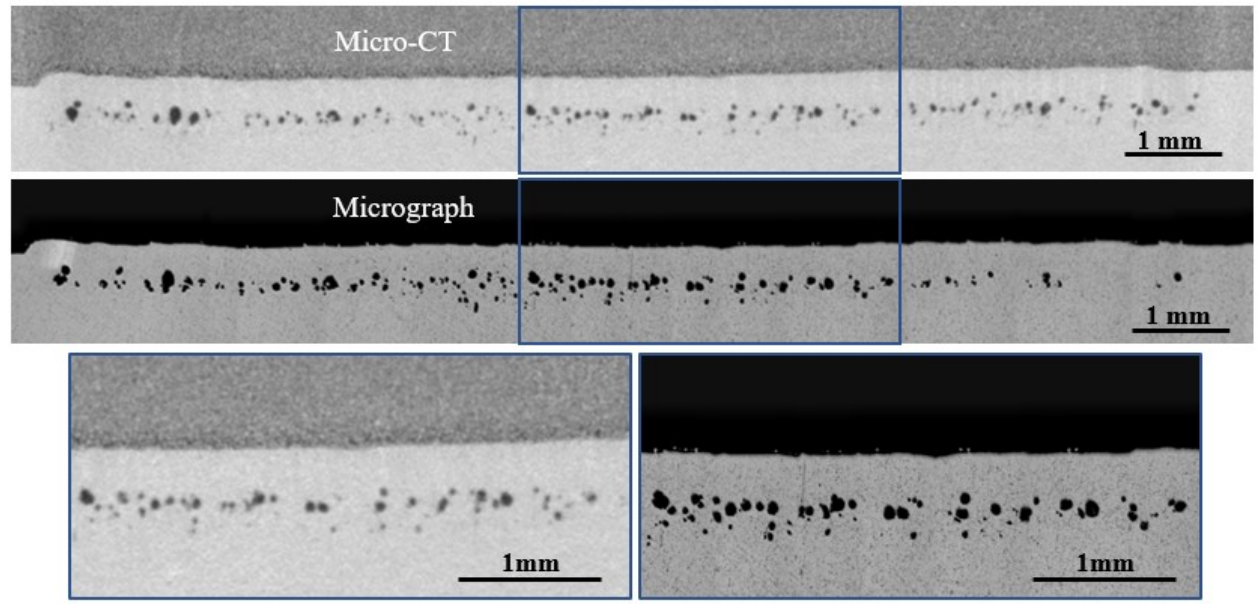

(b)

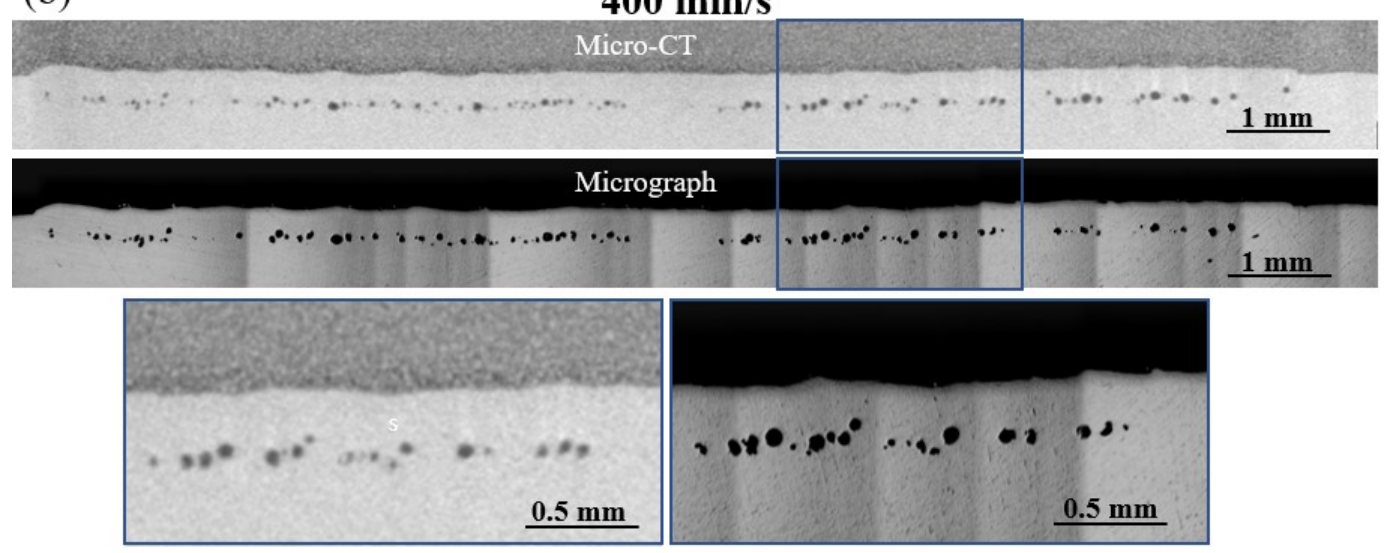

Figure 11.4 2D porosity comparison between micro-CT and micrograph.

\subsection{Conclusions}

A micro-CT scanner is used to measure the keyhole pores formed inside the single tracks fabricated at different locations of the build platform. The results showed that there is a huge variation in the porosity at different locations, which may be due to the undirected flow of inert gas inside the build chamber. Besides, the variation in porosity at different build height is evident but not as significant as the build location. The tracks are formed at the same focal distance, although the build heights are different, 
which may be the reason for the small variation. Moreover, the CT scan measured small pores compared to the micrographs, while missing small pores due to the limitation in the resolution. 


\section{CHAPTER 12 CONCLUSIONS AND FUTURE WORK}

\subsection{Conclusion}

This dissertation elaborates the effect of process parameters on the quality of the parts fabricated with PB-AM processes. The conclusions from this dissertation are:

- The speed of the electron beam affects the temperature gradient, which in turn affects the fluid flow. The differences in the fluid flow, melting and solidification lead to the variation in the surface roughness. In general, the average roughness. Besides, the surface roughness in the transverse direction is higher compared to the scanning direction. Semi-periodic waves are observed in the transverse direction, which significantly contributed to the surface roughness. The numerical model developed using the volume of fluid method captured the surface formation due to the melting and solidification. The temperature melt pool size and roughness predicted by free surface simulation were in good agreement with the experiment.

- The powder scale numerical model showed that the outward flow is observed during the melting process, while the inward flow is observed during the solidifying stage. Besides, the energy density affects the size of the melt pool, and the track width decreases with decreasing laser energy density. The single-track bead height is significantly lower in the simulation compared to the experiment, due to the limited physics and powder height considered. However, the multi-layer simulation showed 
that the proper interlayer bonding is present with $195 \mathrm{~W}$ laser power and $1000 \mathrm{~mm} / \mathrm{s}$ scan speed.

- Keyhole porosity generally increases with increasing LED. Besides, the average porosity and average depth of the pores also increased with increasing LED. However, the limitation of using the LED as a porosity parameter is evident. At the same LED, increasing the laser power has a higher effect. Considering $0.48 \mathrm{~J} / \mathrm{mm}$, at a low power level ( $24 \mathrm{~W}$ and $50 \mathrm{~mm} / \mathrm{s})$, balling formation due to incomplete melting is observed. On the other hand, as the power increase from $96 \mathrm{~W}$ to $195 \mathrm{~W}$, keyhole pores are observed, and the depth of the pores increases with increasing the laser power.

- The laser energy affects the mode of the melting. In the conduction mode melting, the applied energy can only form small depression, which prevents the significant interaction between the surface tension pressure and the evaporation pressure. Besides, in keyhole mode melting, the magnitude of the evaporation pressure is very high which leads to a huge depression. The radius of the keyhole is also a strong function of laser power, and the instability caused by the interaction between the surface tension pressure and the evaporation pressure leads to the formation of the pore. At the same LED, the keyhole depth increased with an increase in the laser power from $100 \mathrm{~W}$ to $300 \mathrm{~W}$, but then slightly decreased when the power was further increased from $300 \mathrm{~W}$ to $400 \mathrm{~W}$, possibly due to the saturation of the laser energy absorption within the keyhole.

- Multilayer porosity results show that the pore number depends on hatch spacing. The number of pores reduced with increasing hatch spacing up to from $60 \%$ to $90 \%$. But 
the increase in hatch spacing from $90 \%$ to $120 \%$ introduced a lack of fusion pores which significantly increased the pore number. Besides, the hatch spacing of $60 \%$ to $90 \%$ did not affect the pore size, while the hatch spacing of $120 \%$ introduced a lack of fusion pores which increased the average pore diameter.

- The mode of melting progressed from incomplete melting to conduction melting, and finally to keyhole melting with an increase in the laser power and scan speed at the same LED. Besides, the depth of the melt pool increased consistently with the increase in laser power. However, the keyhole porosity did not increase with an increase in the melt pool depth, instead, the keyhole porosity increased to a certain level and then decreased with further increase in laser power. The numerical results show that the increase in the melt pool depth is due to the increase in the melt pool temperature and the vapor pressure. Besides, there is a significant increase in penetration with a small increase in laser power due to the exponential relationship between the recoil pressure and the surface temperature. The vapor opening in the conduction mode is stable, and the instability in the keyhole becomes evident with an increase in the depth of the keyhole. The experimental and numerical analysis showed that the LED may not be used as a sole parameter to distinguish the mode of melting as the laser power has a greater effect on melt pool depth than the scan speed. Normalized enthalpy, which includes process parameters as well as material properties, may be more appropriate to explain the shifting from conduction to keyhole mode melting.

- The transient and steady-state regions are identified based on the track width. The track is wider at the initial transient region and has decreasing width at the final 
transient region. The steady region forms between the two transient regions and has a uniform width. However, transient behavior did not have an obvious effect on the depth of the melt pool. The variation in the melt pool size is related to the difference in the thermal gradient in different regions. The numerical study showed that the thermal gradient is higher at the beginning of the scan. Hence initial bump with the wider track is formed because of the strong backflow of the melt pool and pronounced side flow. The tapered shape at the end of the track is due to the semilemniscate shape of the melt pool.

- The effect of residual heat on the melt pool boundary is evident from the scan length effect study. The size of the melt pool increased significantly for a scan length of 0.5 $\mathrm{mm}$. Besides, the length of the melt pool for $195 \mathrm{~W}, 750 \mathrm{~mm} / \mathrm{s}$ is greater than 0.5 $\mathrm{mm}$. Hence, the surface morphology of the $0.5 \mathrm{~mm}$ multi-track showed a single bump. The transverse metallography shows the variation in the melt pool boundary at different locations. The laser turn region showed a significant overlap between the tracks. The overlap is due to the merging of the melt pools from the two successive tracks.

- A single-track porosity measured at different build locations showed that there is a huge variation in the porosity at different locations, which may be due to the undirected flow of inert gas inside the build chamber. Besides, the variation in porosity at different build height is evident but not as significant as the horizontal build location. The tracks are formed at the same focal distance, although the build heights are different, which may be the reason for the small variation. Moreover, the 
CT scan measured small pores compared to the micrographs, while missing small pores due to the limitation in the resolution.

\subsection{Future work}

The new research opportunities are identified based on the work performed for the dissertation. The future works include:

- The inclusion of material properties to obtain the parameter-property chart for different materials is very important. The thermal properties of the materials vary, which affect their response during the laser interaction. Hence, material properties strongly affect melting behavior during the LPBF process. Future studies can be undertaken to develop a property-parameter-performance relationship for different materials.

- During the LPBF process, a negative pressure is developed around the melt pool, which draws the freely moving neighbor particles towards it. This increases the effective layer thickness for the track while eroding the mass for the following track. Such a phenomenon exacerbates the surface roughness and even leads to the mechanical interaction between the part and the recoater blade. The physical parameters that contribute to the negative pressure during the LPBF process may be investigated using a high-fidelity model. The optimization of the build chamber as well as parameters may be performed to minimize the denudation during the LPBF process. 
- The recoil pressure exerted on the melt pool due to evaporation also depends on the chamber pressure. Hence, the numerical investigation may be performed to understand the effect of chamber pressure on the recoil pressure and the subsequent melt pool depression during the melting process. Such a study may help control the formation of the keyhole during the LPBF process.

- The transient zones are formed during the LPBF process. The transient length depends on the initial condition of the powder bed and the melt pool lifetime, which is a function of material property and the laser parameters. The transient lengths may be measured to quantify the effect of laser parameters. Besides, the parameters can be optimized to obtain the small features with higher dimensional accuracy and lower failure rate. 


\section{REFERENCES}

1. Frazier, W.E., Metal additive manufacturing: a review. Journal of Materials Engineering and Performance, 2014. 23(6): p. 1917-1928.

2. $\quad$ Kruth, J.-P., M.-C. Leu, and T. Nakagawa, Progress in additive manufacturing and rapid prototyping. Cirp Annals, 1998. 47(2): p. 525-540.

3. Hu, D. and R. Kovacevic, Sensing, modeling and control for laser-based additive manufacturing. International Journal of Machine Tools and Manufacture, 2003. 43(1): p. 51-60.

4. Rafi, H., et al., Microstructures and mechanical properties of Ti6Al4V parts fabricated by selective laser melting and electron beam melting. Journal of materials engineering and performance, 2013. 22(12): p. 3872-3883.

5. Kasperovich, G., et al., Correlation between porosity and processing parameters in TiAl6V4 produced by selective laser melting. Materials \& Design, 2016. 105: p. 160-170.

6. Ponnusamy, P., et al. Statistical analysis of porosity of 17-4PH alloy processed by selective laser melting. in IOP Conference Series: Materials Science and Engineering. 2017. IOP Publishing.

7. Slotwinski, J.A., E.J. Garboczi, and K.M. Hebenstreit, Porosity measurements and analysis for metal additive manufacturing process control. Journal of research of the National Institute of Standards and Technology, 2014. 119: p. 494.

8. Qi, H., et al., Scanning method of filling lines in electron beam selective melting. Proceedings of the Institution of Mechanical Engineers, Part B: Journal of Engineering Manufacture, 2007. 221(12): p. 1685-1694.

9. Zäh, M.F. and S. Lutzmann, Modelling and simulation of electron beam melting. Production Engineering, 2010. 4(1): p. 15-23.

10. Shen, N. and K. Chou. Thermal modeling of electron beam additive manufacturing process: Powder sintering effects. in ASME 2012 International Manufacturing Science and Engineering Conference collocated with the 40th North American Manufacturing Research Conference and in participation with the International Conference on Tribology Materials and Processing. 2012. American Society of Mechanical Engineers.

11. Cheng, B., et al., On Process Temperature in Powder-Bed Electron Beam Additive Manufacturing: Model Development and Validation. Journal of Manufacturing Science and Engineering, 2014. 136(6): p. 061018.

12. Price, S., et al., On Process Temperature in Powder-Bed Electron Beam Additive Manufacturing: Process Parameter Effects. Journal of Manufacturing Science and Engineering, 2014. 136(6): p. 061019.

13. Galati, M., et al., Modelling energy source and powder properties for the development of a thermal FE model of the EBM additive manufacturing process. Additive Manufacturing, 2017. 14: p. 49-59. 
14. Jamshidinia, M., F. Kong, and R. Kovacevic, Numerical Modeling of Heat Distribution in the Electron Beam Melting ${ }^{\circledR}$ of Ti-6Al-4V. Journal of Manufacturing Science and Engineering, 2013. 135(6): p. 061010.

15. Körner, C., E. Attar, and P. Heinl, Mesoscopic simulation of selective beam melting processes. Journal of Materials Processing Technology, 2011. 211(6): p. 978-987.

16. Scharowsky, T., et al. Observation and numerical simulation of melt pool dynamic and beam powder interaction during selective electron beam melting. in Proceedings of the Solid Freeform Fabrication Symposium. 2012.

17. Ammer, R., et al., Simulating fast electron beam melting with a parallel thermal free surface lattice Boltzmann method. Computers \& Mathematics with Applications, 2014. 67(2): p. 318-330.

18. Yan, W., et al., Multi-physics modeling of single/multiple-track defect mechanisms in electron beam selective melting. Acta Materialia, 2017. 134: p. 324-333.

19. Tran, H.-C. and Y.-L. Lo, Heat transfer simulations of selective laser melting process based on volumetric heat source with powder size consideration. Journal of Materials Processing Technology, 2018. 255: p. 411-425.

20. $\mathrm{Li}, \mathrm{Y}$. and D. Gu, Parametric analysis of thermal behavior during selective laser melting additive manufacturing of aluminum alloy powder. Materials \& Design, 2014. 63: p. 856-867.

21. Foroozmehr, A., M. Badrossamay, and E. Foroozmehr, Finite element simulation of selective laser melting process considering optical penetration depth of laser in powder bed. Materials \& Design, 2016. 89: p. 255-263.

22. Dai, D. and D. Gu, Tailoring surface quality through mass and momentum transfer modeling using a volume of fluid method in selective laser melting of TiC/AlSilOMg powder. International Journal of Machine Tools and Manufacture, 2015. 88: p. 95-107.

23. Xia, M., et al., Influence of hatch spacing on heat and mass transfer, thermodynamics and laser processability during additive manufacturing of Inconel 718 alloy. International Journal of Machine Tools and Manufacture, 2016. 109: p. 147-157.

24. Khairallah, S.A. and A. Anderson, Mesoscopic simulation model of selective laser melting of stainless steel powder. Journal of Materials Processing Technology, 2014. 214(11): p. 2627-2636.

25. Lee, Y. and W. Zhang, Modeling of heat transfer, fluid flow and solidification microstructure of nickel-base superalloy fabricated by laser powder bed fusion. Additive Manufacturing, 2016. 12: p. 178-188.

26. Wu, Y.-C., et al., Numerical modeling of melt-pool behavior in selective laser melting with random powder distribution and experimental validation. Journal of Materials Processing Technology, 2018. 254: p. 72-78.

27. Leitz, K.-H., et al., Multi-physical simulation of selective laser melting. 2017. 72(5): p. 331-338.

28. Russell, M., A. Souto-Iglesias, and T. Zohdi, Numerical simulation of Laser Fusion Additive Manufacturing processes using the SPH method. Computer Methods in Applied Mechanics and Engineering, 2018. 341: p. 163-187. 
29. Weirather, J., et al., A Smoothed Particle Hydrodynamics Model for Laser Beam Melting of Ni-based Alloy 718. Computers \& Mathematics with Applications, 2018.

30. Aboulkhair, N.T., et al., Reducing porosity in AlSil0Mg parts processed by selective laser melting. Additive Manufacturing, 2014. 1: p. 77-86.

31. Cherry, J., et al., Investigation into the effect of process parameters on microstructural and physical properties of $316 \mathrm{~L}$ stainless steel parts by selective laser melting. The International Journal of Advanced Manufacturing Technology, 2015. 76(5-8): p. 869-879.

32. Edwards, P. and M. Ramulu, Fatigue performance evaluation of selective laser melted Ti-6Al-4V. Materials Science and Engineering: A, 2014. 598: p. 327-337.

33. Leuders, S., et al., On the mechanical behaviour of titanium alloy TiAl6V4 manufactured by selective laser melting: Fatigue resistance and crack growth performance. International Journal of Fatigue, 2013. 48: p. 300-307.

34. Siddique, S., et al., Computed tomography for characterization of fatigue performance of selective laser melted parts. Materials \& Design, 2015. 83: p. 661-669.

35. Gong, H., et al., Analysis of defect generation in Ti-6Al-4V parts made using powder bed fusion additive manufacturing processes. Additive Manufacturing, 2014. 1: p. 87-98.

36. King, W.E., et al., Observation of keyhole-mode laser melting in laser powderbed fusion additive manufacturing. Journal of Materials Processing Technology, 2014. 214(12): p. 2915-2925.

37. Zhou, X., et al., 3D-imaging of selective laser melting defects in a $\mathrm{Co}-\mathrm{Cr}-\mathrm{Mo}$ alloy by synchrotron radiation micro-CT. Acta Materialia, 2015. 98: p. 1-16.

38. Ziółkowski, G., et al., Application of X-ray CT method for discontinuity and porosity detection in $316 \mathrm{~L}$ stainless steel parts produced with SLM technology. Archives of Civil and Mechanical Engineering, 2014. 14(4): p. 608-614.

39. Kim, T.B., et al., Additive manufactured porous titanium structures: Throughprocess quantification of pore and strut networks. Journal of Materials Processing Technology, 2014. 214(11): p. 2706-2715.

40. Van Bael, S., et al., Micro-CT-based improvement of geometrical and mechanical controllability of selective laser melted Ti6Al4V porous structures. Materials Science and Engineering: A, 2011. 528(24): p. 7423-7431.

41. Mahale, T.R., Electron beam melting of advanced materials and structures. 2009.

42. Oksman, P., et al., The Effective Thermal Conductivity Method in Continuous Casting of Steel. Acta Polytechnica Hungarica, 2014. 11(9): p. 6-22.

43. Chan, K.S., et al., Fatigue life of titanium alloys fabricated by additive layer manufacturing techniques for dental implants. Metallurgical and Materials Transactions A, 2013. 44(2): p. 1010-1022.

44. Koike, M., et al., Evaluation of titanium alloy fabricated using electron beam melting system for dental applications. Journal of Materials Processing Technology, 2011. 211(8): p. 1400-1408.

45. Gong, X., T. Anderson, and K. Chou. Review on powder-based electron beam additive manufacturing technology. in ASME/ISCIE 2012 International 
Symposium on Flexible Automation. 2012. American Society of Mechanical Engineers.

46. Safdar, A., et al., Effect of process parameters settings and thickness on surface roughness of EBM produced Ti-6Al-4V. Rapid Prototyping Journal, 2012. 18(5): p. 401-408.

47. Guo, C., W. Ge, and F. Lin, Effects of scanning parameters on material deposition during Electron Beam Selective Melting of Ti-6Al-4V powder. Journal of Materials Processing Technology, 2015. 217: p. 148-157.

48. Strano, G., et al., Surface roughness analysis, modelling and prediction in selective laser melting. Journal of Materials Processing Technology, 2013. 213(4): p. 589-597.

49. Körner, C., A. Bauereiß, and E. Attar, Fundamental consolidation mechanisms during selective beam melting of powders. Modelling and Simulation in Materials Science and Engineering, 2013. 21(8): p. 085011.

50. Bauereiß, A., T. Scharowsky, and C. Körner, Defect generation and propagation mechanism during additive manufacturing by selective beam melting. Journal of Materials Processing Technology, 2014. 214(11): p. 2522-2528.

51. Welsch, G., R. Boyer, and E. Collings, Materials properties handbook: titanium alloys. 1993: ASM international.

52. Rouquette, S., J. Guo, and P. Le Masson, Estimation of the parameters of a Gaussian heat source by the Levenberg-Marquardt method: application to the electron beam welding. International Journal of Thermal Sciences, 2007. 46(2): p. $128-138$.

53. Gaytan, S., et al., Advanced metal powder based manufacturing of complex components by electron beam melting. Materials Technology, 2009. 24(3): p. 180190.

54. Yang, J., et al., Experimental investigation and $3 D$ finite element prediction of the heat affected zone during laser assisted machining of Ti6Al4V alloy. Journal of Materials Processing Technology, 2010. 210(15): p. 2215-2222.

55. Elmer, J., et al., Phase transformation dynamics during welding of Ti-6Al-4V. Journal of applied physics, 2004. 95(12): p. 8327-8339.

56. Yang, M., Z. Yang, and B. Qi, Effect of fluid in molten pool on the welds with Ti6 Al- $4 \mathrm{~V}$ during pulsed arc welding. The International Journal of Advanced Manufacturing Technology, 2015. 81(5-8): p. 1007-1016.

57. Price, S., et al. Temperature measurements in powder-bed electron beam additive manufacturing. in ASME 2014 International Mechanical Engineering Congress and Exposition. 2014. American Society of Mechanical Engineers.

58. Kruth, J.-P., et al., Selective laser melting of iron-based powder. Journal of Materials Processing Technology, 2004. 149(1): p. 616-622.

59. Yadroitsev, I., P. Bertrand, and I. Smurov, Parametric analysis of the selective laser melting process. Applied surface science, 2007. 253(19): p. 8064-8069.

60. Childs, T., C. Hauser, and M. Badrossamay, Mapping and modelling single scan track formation in direct metal selective laser melting. CIRP AnnalsManufacturing Technology, 2004. 53(1): p. 191-194. 
61. Li, R., et al., Balling behavior of stainless steel and nickel powder during selective laser melting process. The International Journal of Advanced Manufacturing Technology, 2012. 59(9-12): p. 1025-1035.

62. Dilip, J., et al. A short study on the fabrication of single track deposits in SLM and characterization. in Solid freeform fabrication symposium. 2016.

63. Panwisawas, C., et al., Mesoscale modelling of selective laser melting: Thermal fluid dynamics and microstructural evolution. Computational Materials Science, 2017. 126: p. 479-490.

64. Khairallah, S.A., et al., Laser powder-bed fusion additive manufacturing: physics of complex melt flow and formation mechanisms of pores, spatter, and denudation zones. Acta Materialia, 2016. 108: p. 36-45.

65. Panwisawas, C., et al., On the role of thermal fluid dynamics into the evolution of porosity during selective laser melting. Scripta Materialia, 2015. 105: p. 14-17.

66. Shrestha, S. and K. Chou, A Build Surface Study of Powder-Bed Electron Beam Additive Manufacturing by 3D Thermo-fluid Simulation and White-light Interferometry. International Journal of Machine Tools and Manufacture, 2017.

67. Zhou, J., Y. Zhang, and J. Chen, Numerical simulation of random packing of spherical particles for powder-based additive manufacturing. Journal of Manufacturing Science and Engineering, 2009. 131(3): p. 031004.

68. Kobayashi, M., et al., System for measuring the spectral distribution of normal emissivity of metals with direct current heating. International journal of thermophysics, 1999. 20(1): p. 289-298.

69. Matthews, M.J., et al., Denudation of metal powder layers in laser powder bed fusion processes. Acta Materialia, 2016. 114: p. 33-42.

70. Aboulkhair, N.T., et al., On the formation of AlSi10Mg single tracks and layers in selective laser melting: Microstructure and nano-mechanical properties. Journal of Materials Processing Technology, 2016. 230: p. 88-98.

71. Brandl, E., et al., Additive manufactured AlSi10Mg samples using Selective Laser Melting (SLM): Microstructure, high cycle fatigue, and fracture behavior. Materials \& Design, 2012. 34: p. 159-169.

72. Amato, K., et al., Microstructures and mechanical behavior of Inconel 718 fabricated by selective laser melting. Acta Materialia, 2012. 60(5): p. 2229-2239.

73. Jia, Q. and D. Gu, Selective laser melting additive manufactured Inconel 718 superalloy parts: High-temperature oxidation property and its mechanisms. Optics \& Laser Technology, 2014. 62: p. 161-171.

74. Song, B., et al., Effects of processing parameters on microstructure and mechanical property of selective laser melted Ti6Al4V. Materials \& Design, 2012. 35: p. 120-125.

75. Guan, K., et al., Effects of processing parameters on tensile properties of selective laser melted 304 stainless steel. Materials \& Design, 2013. 50: p. 581-586.

76. Vandenbroucke, B. and J.-P. Kruth, Selective laser melting of biocompatible metals for rapid manufacturing of medical parts. Rapid Prototyping Journal, 2007. 13(4): p. 196-203.

77. Murr, L., et al., Microstructure and mechanical behavior of Ti-6Al-4V produced by rapid-layer manufacturing, for biomedical applications. Journal of the mechanical behavior of biomedical materials, 2009. 2(1): p. 20-32. 
78. Yadroitsev, I., P. Krakhmalev, and I. Yadroitsava, Selective laser melting of Ti6Al4V alloy for biomedical applications: Temperature monitoring and microstructural evolution. Journal of Alloys and Compounds, 2014. 583: p. 404409.

79. Wycisk, E., et al. High cycle fatigue (HCF) performance of Ti-6Al-4V alloy processed by selective laser melting. in Advanced materials research. 2013. Trans Tech Publ.

80. Mercelis, P. and J.-P. Kruth, Residual stresses in selective laser sintering and selective laser melting. Rapid Prototyping Journal, 2006. 12(5): p. 254-265.

81. Cheng, B., S. Shrestha, and Y.K. Chou. Stress and deformation evaluations of scanning strategy effect in selective laser melting. in ASME 2016 11th International Manufacturing Science and Engineering Conference. 2016. American Society of Mechanical Engineers.

82. Yadroitsev, I., et al., Single track formation in selective laser melting of metal powders. Journal of Materials Processing Technology, 2010. 210(12): p. 16241631.

83. Read, N., et al., Selective laser melting of AlSi10Mg alloy: Process optimisation and mechanical properties development. Materials \& Design (1980-2015), 2015. 65: p. 417-424.

84. Bertoli, U.S., et al., On the limitations of volumetric energy density as a design parameter for selective laser melting. Materials \& Design, 2017. 113: p. 331-340.

85. Pang, S., W. Chen, and W. Wang, A quantitative model of keyhole instability induced porosity in laser welding of titanium alloy. Metallurgical and Materials Transactions A, 2014. 45(6): p. 2808-2818.

86. Cho, J.-H. and S.-J. Na, Implementation of real-time multiple reflection and Fresnel absorption of laser beam in keyhole. Journal of Physics D: Applied Physics, 2006. 39(24): p. 5372.

87. Gong, H., et al. Melt pool characterization for selective laser melting of Ti-6Al-4V pre-alloyed powder. in Solid freeform fabrication symposium. 2014.

88. Trapp, J., et al., In situ absorptivity measurements of metallic powders during laser powder-bed fusion additive manufacturing. Applied Materials Today, 2017. 9: p. 341-349.

89. Hann, D., J. Iammi, and J. Folkes, A simple methodology for predicting laserweld properties from material and laser parameters. Journal of Physics D: Applied Physics, 2011. 44(44): p. 445401.

90. Kroos, J., U. Gratzke, and G. Simon, Towards a self-consistent model of the keyhole in penetration laser beam welding. Journal of physics D: Applied physics, 1993. 26(3): p. 474.

91. Wang, Y., et al., A processing diagram for high-density Ti-6Al-4V by selective laser melting. Rapid Prototyping Journal, 2018.

92. Tan, J., C. Tang, and C. Wong, A Computational Study on Porosity Evolution in Parts Produced by Selective Laser Melting. Metallurgical and Materials Transactions A, 2018: p. 1-11.

93. Zhao, C., et al., Real-time monitoring of laser powder bed fusion process using high-speed X-ray imaging and diffraction. Scientific reports, 2017. 7(1): p. 3602. 
94. Parab, N.D., et al., Ultrafast X-ray imaging of laser-metal additive manufacturing processes. Journal of synchrotron radiation, 2018. 25(5).

95. Cunningham, R., et al., Keyhole threshold and morphology in laser melting revealed by ultrahigh-speed $x$-ray imaging. Science, 2019. 363(6429): p. 849-852.

96. Shrestha, S. and K. Chou. Individual and Coupled Contributions of Laser Power and Scanning Speed towards Process-Induced Porosity in Selective Laser Melting. in Solid freeform fabrication symposium. 2018.

97. Ye, J., et al. Laser Absorption and Scaling Behavior in Powder Bed Fusion Additive Manufacturing of Metals. in CLEO: Science and Innovations. 2018. Optical Society of America.

98. Mishra, B. and R.K. Rajamani, The discrete element method for the simulation of ball mills. Applied Mathematical Modelling, 1992. 16(11): p. 598-604.

99. Yan, W., et al., Meso-scale modeling of multiple-layer fabrication process in selective electron beam melting: inter-layer/track voids formation. Materials \& Design, 2018. 141: p. 210-219.

100. Kloss, C., et al., Models, algorithms and validation for opensource DEM and $C F D-D E M$. Progress in Computational Fluid Dynamics, an International Journal, 2012. 12(2-3): p. 140-152.

101. Escano, L.I., et al., Revealing particle-scale powder spreading dynamics in powder-bed-based additive manufacturing process by high-speed $x$-ray imaging. Scientific reports, 2018. 8(1): p. 15079.

102. Dilip, J., et al., Influence of processing parameters on the evolution of melt pool, porosity, and microstructures in Ti-6Al-4V alloy parts fabricated by selective laser melting. Progress in Additive Manufacturing, 2017. 2(3): p. 157-167.

103. Shrestha, S. and K. Chou, Single track scanning experiment in laser powder bed fusion process. Procedia Manufacturing, 2018. 26: p. 857-864.

104. Martin, A., et al., Ultrafast dynamics of laser-metal interactions in additive manufacturing alloys captured by in situ X-ray imaging. Materials Today Advances, 2019. 1: p. 100002.

105. Shrestha, S. and K. Chou. Porosity Analysis in Metal Additive Manufacturing by Micro-CT. in ASME 2018 International Mechanical Engineering Congress and Exposition. 2018. American Society of Mechanical Engineers.

106. Shrestha, S., T. Starr, and K. Chou, A Study of Keyhole Porosity in Selective Laser Melting: Single Track Scanning with Micro-CT Analysis. Journal of Manufacturing Science and Engineering, 2019: p. 1-23.

107. Shrestha, S. and K. Chou, A Numerical Study on the Keyhole Formation During Laser Powder Bed Fusion Process. Journal of Manufacturing Science and Engineering, 2019.

108. Galarraga, H., et al., Effects of the microstructure and porosity on properties of Ti-6Al-4V ELI alloy fabricated by electron beam melting (EBM). Additive Manufacturing, 2016. 10: p. 47-57.

109. Maskery, I., et al., Quantification and characterisation of porosity in selectively laser melted Al-Si10-Mg using X-ray computed tomography. Materials Characterization, 2016. 111: p. 193-204.

110. Wits, W.W., et al., Porosity testing methods for the quality assessment of selective laser melted parts. CIRP annals, 2016. 65(1): p. 201-204. 
111. Dong, Z., et al., Effect of hatch spacing on melt Pool and as-built quality during selective laser melting of stainless steel: modeling and experimental approaches. Materials, 2019. 12(1): p. 50.

112. Volpp, J., F. Brueckner, and A.F. Kaplan, Track geometry variations in selective laser melting processes. Journal of Laser Applications, 2019. 31(2): p. 022310.

113. Thijs, L., et al., A study of the microstructural evolution during selective laser melting of Ti-6Al-4V. Acta Materialia, 2010. 58(9): p. 3303-3312.

114. Gu, D., et al., Densification behavior, microstructure evolution, and wear performance of selective laser melting processed commercially pure titanium. Acta Materialia, 2012. 60(9): p. 3849-3860.

115. Ma, M., et al., Layer thickness dependence of performance in high-power selective laser melting of $1 \mathrm{Cr} 18 \mathrm{Ni} 9 \mathrm{Ti}$ stainless steel. Journal of Materials Processing Technology, 2015. 215: p. 142-150.

116. Wang, Z., et al., The microstructure and mechanical properties of depositedIN718 by selective laser melting. Journal of Alloys and Compounds, 2012. 513: p. 518-523.

117. Lu, Y., et al., Study on the microstructure, mechanical property and residual stress of SLM Inconel-718 alloy manufactured by differing island scanning strategy. Optics \& Laser Technology, 2015. 75: p. 197-206.

118. Monzón, M., et al., Standardization in additive manufacturing: activities carried out by international organizations and projects. The international journal of advanced manufacturing technology, 2015. 76(5-8): p. 1111-1121.

119. Zhang, B., L. Dembinski, and C. Coddet, The study of the laser parameters and environment variables effect on mechanical properties of high compact parts elaborated by selective laser melting $316 \mathrm{~L}$ powder. Materials Science and Engineering: A, 2013. 584: p. 21-31.

120. Lee, S., et al., Data analytics approach for melt-pool geometries in metal additive manufacturing. Science and Technology of Advanced Materials, 2019(justaccepted).

121. Martin, A.A., et al., Dynamics of pore formation during laser powder bed fusion additive manufacturing. Nature communications, 2019. 10(1): p. 1-10.

122. Thanki, A., et al., Study of keyhole-porosities in selective laser melting using Xray computed tomography. Proceedings of iCT 2019, 2019: p. 1-7.

123. Hann, D., J. Iammi, and J. Folkes. Keyholing or conduction-Prediction of laser penetration depth. in Proceedings of the 36th International MATADOR Conference. 2010. Springer.

124. Gunenthiram, V., et al., Analysis of laser-melt pool-powder bed interaction during the selective laser melting of a stainless steel. 2017.

125. Bidare, P., et al., Fluid and particle dynamics in laser powder bed fusion. Acta Materialia, 2018. 142: p. 107-120.

126. Meier, C., et al., Modeling and characterization of cohesion in fine metal powders with a focus on additive manufacturing process simulations. Powder technology, 2019. 343: p. 855-866.

127. Wischeropp, T.M., et al., Measurement of actual powder layer height and packing density in a single layer in selective laser melting. Additive Manufacturing, 2019. 
128. Yan, C., et al., Evaluations of cellular lattice structures manufactured using selective laser melting. International Journal of Machine Tools and Manufacture, 2012. 62: p. 32-38.

129. Xiao, Z., et al., Evaluation of topology-optimized lattice structures manufactured via selective laser melting. Materials \& Design, 2018. 143: p. 27-37.

130. Yadroitsev, I. and I. Smurov, Surface morphology in selective laser melting of metal powders. Physics Procedia, 2011. 12: p. 264-270.

131. Pupo, Y., et al., Scanning space analysis in selective laser melting for CoCrMo powder. Procedia Engineering, 2013. 63: p. 370-378.

132. $\mathrm{Hu}, \mathrm{Z}$., et al., Contact angle evolution during selective laser melting. Materials \& Design, 2018. 139: p. 304-313.

133. Criales, L.E., et al., Laser powder bed fusion of nickel alloy 625: Experimental investigations of effects of process parameters on melt pool size and shape with spatter analysis. International Journal of Machine Tools and Manufacture, 2017.

134. Criales, L.E., et al., Predictive modeling and optimization of multi-track processing for laser powder bed fusion of nickel alloy 625. Additive Manufacturing, 2017. 13: p. 14-36.

135. Bayat, M., S. Mohanty, and J.H. Hattel, Multiphysics modelling of lack-of-fusion voids formation and evolution in IN718 made by multi-track/multi-layer L-PBF. International Journal of Heat and Mass Transfer, 2019. 139: p. 95-114.

136. Gu, H., et al., Multi-physics modelling of molten pool development and track formation in multi-track, multi-layer and multi-material selective laser melting. International Journal of Heat and Mass Transfer, 2020. 151: p. 119458.

137. Parry, L., I. Ashcroft, and R.D. Wildman, Understanding the effect of laser scan strategy on residual stress in selective laser melting through thermo-mechanical simulation. Additive Manufacturing, 2016. 12: p. 1-15.

138. Francis, Z.R., The effects of laser and electron beam spot size in additive manufacturing processes. 2017, Carnegie Mellon University.

139. Liu, B., et al., Investigation the effect of particle size distribution on processing parameters optimisation in selective laser melting process. Additive manufacturing research group, Loughborough University, 2011: p. 227-238.

140. Sufiiarov, V.S., et al., The effect of layer thickness at selective laser melting. Procedia engineering, 2017. 174: p. 126-134.

141. Ferrar, B., et al., Gas flow effects on selective laser melting (SLM) manufacturing performance. Journal of Materials Processing Technology, 2012. 212(2): p. 355364.

142. Seifi, M., et al., Overview of materials qualification needs for metal additive manufacturing. Jom, 2016. 68(3): p. 747-764.

143. Wang, F., Mechanical property study on rapid additive layer manufacture Hastelloy ${ }^{\circledR} X$ alloy by selective laser melting technology. The International Journal of Advanced Manufacturing Technology, 2012. 58(5-8): p. 545-551.

144. Zhang, S., et al., An experimental study of tensile property variability in selective laser melting. Journal of Manufacturing Processes, 2019. 43: p. 26-35.

145. Fitzgerald, E. and W. Everhart. The effect of location on the structure and mechanical properties of selective laser melted $316 \mathrm{~L}$ stainless steel. in 
Proceedings of the 27th Annual International Solid Freeform Fabrication Symposium. 2016.

146. Lewandowski, J.J. and M. Seifi, Metal additive manufacturing: a review of mechanical properties. Annual Review of Materials Research, 2016. 46: p. 151186.

147. Ladewig, A., et al., Influence of the shielding gas flow on the removal of process by-products in the selective laser melting process. Additive Manufacturing, 2016. 10: p. 1-9.

148. Kong, C.-J., et al. High density Ti6Al4V via SLM processing: microstructure and mechanical properties. in International Solid Freeform Fabrication Symposium. 2011. 


\section{APPENDIX A BUILD LOCATION EFFECT ON THE SINGLE-TRACK PORE CHARACTERISTICS FORMED WITH SELECTIVE LAESR MELTING PROCESS}

The LPBF process utilizes galvanometer laser beam scanning system to control the location of the laser in the build platform. Figure A.1 shows the schematic of scanning system with major components. The laser source is incident on the galvanometer-controlled mirrors, which moves the laser beam in the desired pattern, and F-theta lens is used to focus the lens onto the build platform. F-theta lens provides a plane focusing surface in contrast to the standard lenses which focus the laser beam on the spherical surface. Although, theoretically the combination of galvo mirrors and F-theta lens can result in almost constant spot size and scanning speed across the scan field, there may be some variation which still needs to be investigated to ensure the quality of the part is uniform throughout the build platform in the LPBF process. 


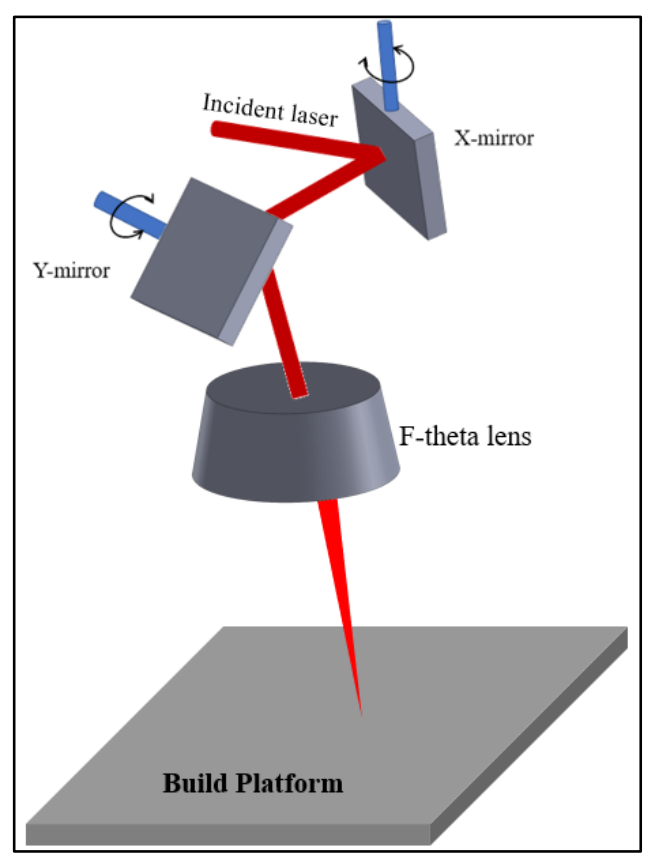

Figure A.1 Schematic of laser control in EOS M270.

Figure A.2(a) shows a typical reconstructed cylinder sample fabricated at the build height of $6.6 \mathrm{~mm}$. Hence, the upper and lower surfaces have support structures attached to it, which was removed by polishing. The four single tracks formed on top of the semi-cylinder base is shown in Figure A.2(b). The difference in the characteristics between the tracks is evident as the width of the track varied with the process parameters. Besides, the 2D slice taken at the center of $195 \mathrm{~W}$ and $200 \mathrm{~mm} / \mathrm{s}$ track is also presented. Three different regions: solid, powder and pore are distinguished based on the grayscale image. The light gray color represents the solid Ti-6Al-4V, the dark gray color represents the powder contained within the cylinder and the black region represents the void. The significant number of pores are formed with $195 \mathrm{~W}$ and $200 \mathrm{~mm} / \mathrm{s}$. 


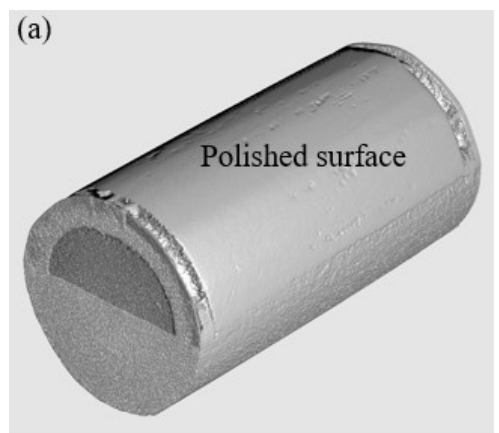

(b)
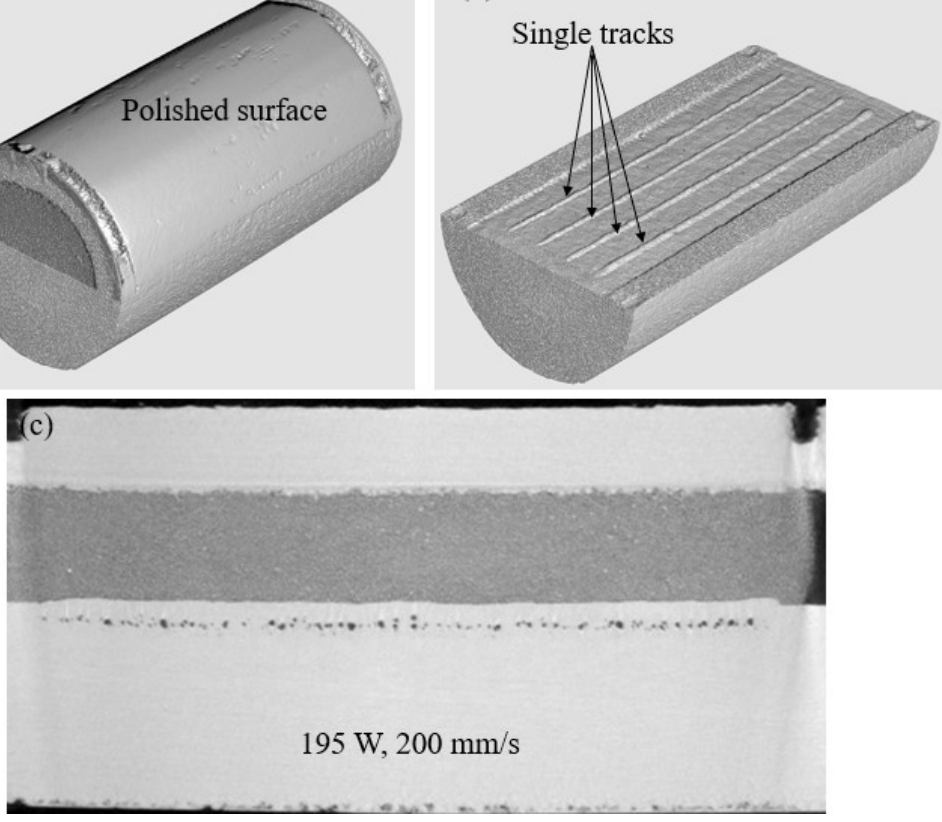

Figure A.2 CT scan results of (a) Cylinder sample (b) Single track morphology, and (c) 2D slice image showing the pores formed with $195 \mathrm{~W}$ and $200 \mathrm{~mm} / \mathrm{s}$.

The track width and depth are a function of laser parameters, that is laser power, scan speed, and beam spot size. Besides, the depth of the pores may also be related to the laser power and scan speed. The depth of the pores can be estimated using the transverse profile as shown in Figure A.3. Besides, the 3D pores formed beneath the single-track surface is also presented through Figure A.3(c). The formation of the keyhole pores would depend on the instabilities in the keyhole wall, which is triggered by the recoil pressure and surface tension pressure. The recoil pressure, which is a function of melt pool temperature is highly affected by the laser parameters. The keyhole pores formed are mostly spherical. 

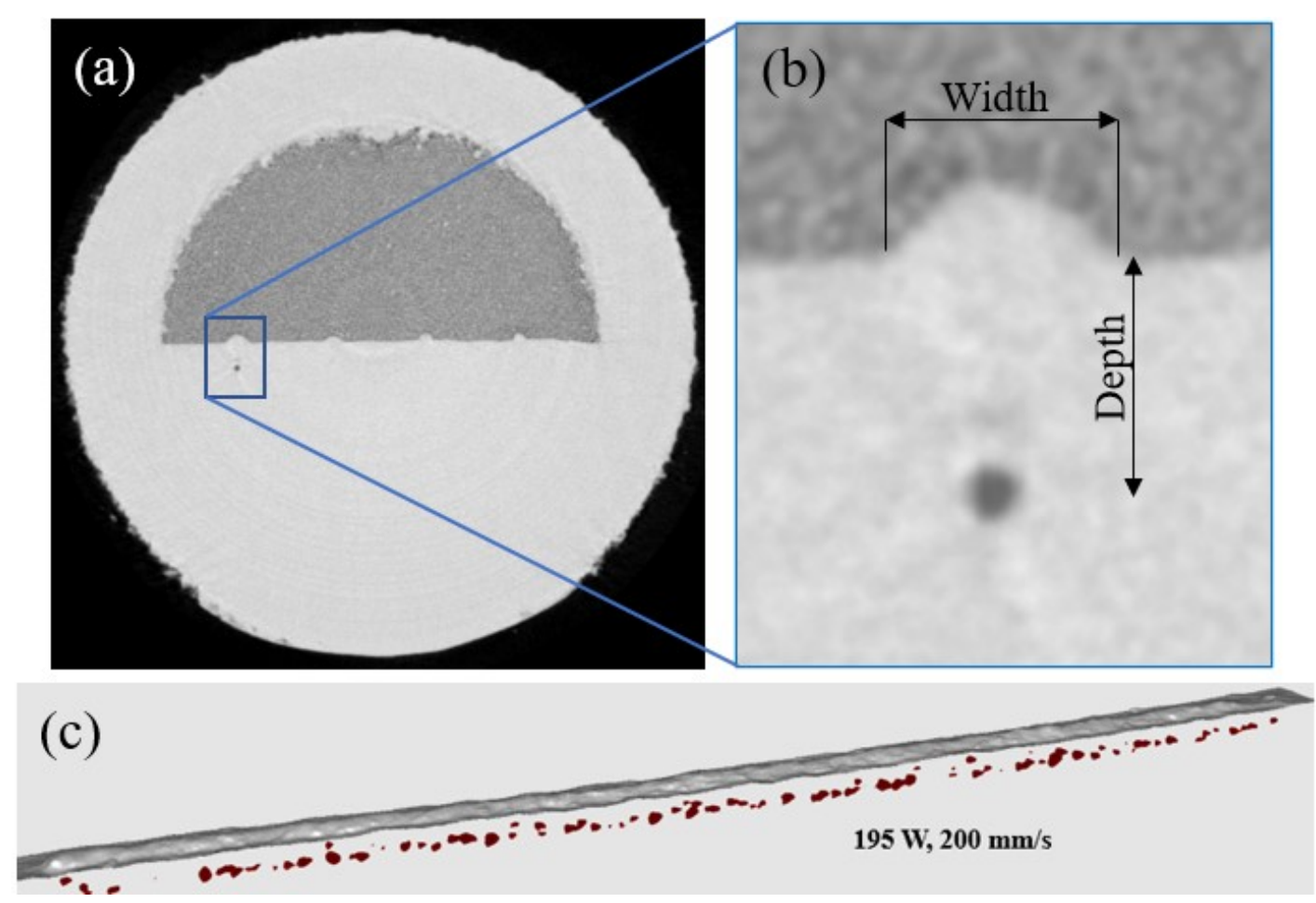

Figure A.3 (a) Transverse section, (b) zoom in view of the track bump and pore, and (c) $3 \mathrm{D}$ pores formed beneath the track surface.

The pore distribution in the longitudinal direction at the center of the melt pool is obtained by polishing. The methodology followed to obtain the micrographs is explained through Figure A.3. The sample is first sectioned to remove all the powder contained inside the cylinder and reveal the single tracks formed within. The sample is then cut close to the desired track, mounted and polished to observe the pores formed along the scan track. The longitudinal profile showing the pores formed due to $195 \mathrm{~W}$ and 200 $\mathrm{mm} / \mathrm{s}$ is shown. 


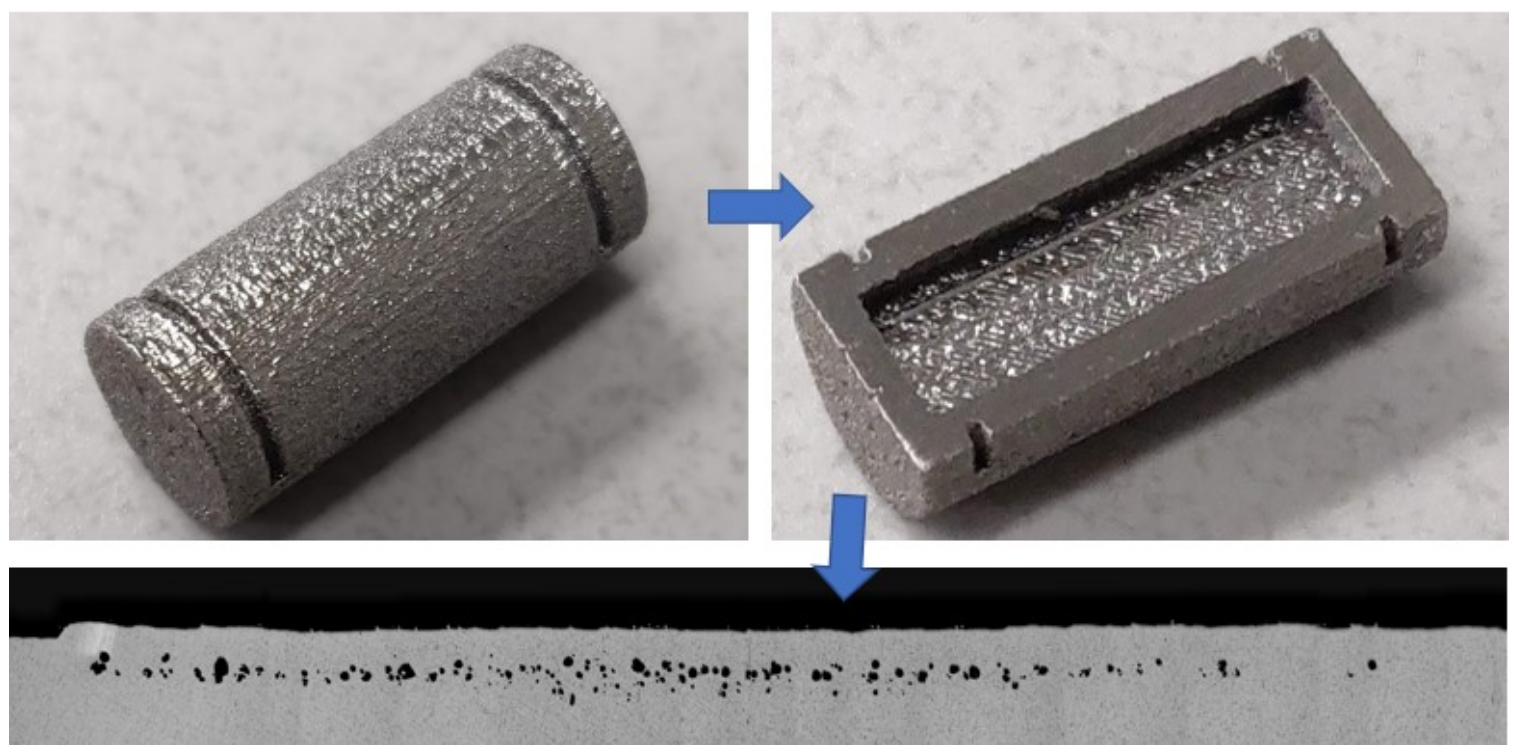

Figure A.4 Steps followed to obtain micrographs of the pore distribution. 


\title{
CURRICULUM VITAE
}

\author{
Subin Shrestha
}

Department of Mechanical Engineering

J.B. Speed School of Engineering

University of Louisville

Louisville, KY 40292

\section{EDUCATION}

PhD, Department of Mechanical Engineering, University of Louisville, Louisville, KY,

Dissertation Title: Experimental and Numerical Study of Process Parameters Effects Towards Part Quality and Porosity During Powder Bed Additive Manufacturing Processes.

Adviser: Dr. Kevin Chou, Professor, Industrial Engineering Department, University of Louisville, KY, USA

Master's in Engineering, Department of Mechanical Engineering, University of Louisville, Louisville, KY

PhD student, Department of Mechanical Engineering, University of Alabama, Tuscaloosa, AL, USA

Bachelor's in Mechanical Engineering, Institute of Engineering, Pulchowk Campus, Lalitpur, Nepal

\section{RESEARCH EXPERIENCE}

01/2017-08/2020 Research Assistant, University of Louisville

- Fabricated specimens for research on laser powder bed fusion process (LPBF) using EOS M270.

- Used Bruker micro-CT to characterize pores formed due to user parameter settings during LPBF process. 
- Developed a discrete element model (DEM) to simulate the powder spreading process.

- Developed a high-fidelity thermo-fluid model to understand the flow dynamics during the laser melting.

- Performed metallography of single-track and multiple track samples to observe the melt pool boundary along the scan direction and transverse direction.

08/2015 - 12/2016 Research Assistant, University of Alabama

- Used Hot Disk TPS $2500 \mathrm{~S}$ to estimate the metal powder thermal conductivity from $20^{\circ} \mathrm{C}$ to $900^{\circ} \mathrm{C}$.

- Developed a thermo-fluid model to investigate the surface formation during raster scanning in an electron beam additive manufacturing process.

- Used the finite element model to predict the residual heat and distortion during the LPBF process.

12/2013 - 07/2015 3D CAD and System Engineer, E\&T Nepal Pvt. Ltd.

- Used moving particle semi-implicit method to predict the fluid flow around the car.

- Worked in a team as a developer to add lift and drag force calculations to an in-house code.

- Worked as a team leader to add fluid-structure interaction module to an in-house code.

TEACHING EXPERIENCE

08/2014-10/2014 Teaching Assistant, Finite Element Method, Institute of Engineering, Pulchowk Campus, Tribhuvan University, Nepal

\section{REFERRED JOURNAL ARTICLES}

1. Shrestha, S. and Kevin Chou, Y., 2019. A Numerical Study on the Keyhole Formation During Laser Powder Bed Fusion Process. Journal of Manufacturing Science and Engineering, 141(10).

2. Zhang, S., Rauniyar, S., Shrestha, S., Ward, A. and Chou, K., 2019. An experimental study of tensile property variability in selective laser melting. Journal of Manufacturing Processes, 43, pp.26-35.

3. Shrestha, S., Starr, T. and Chou, K., 2019. A Study of Keyhole Porosity in Selective Laser Melting: Single-Track Scanning with Micro-CT Analysis. Journal of Manufacturing Science and Engineering, 141(7), p.071004. 
4. Shrestha, S., Rauniyar, S. and Chou, K., 2019. Thermo-Fluid Modeling of Selective Laser Melting: Single-Track Formation Incorporating Metallic Powder. Journal of Materials Engineering and Performance, 28(2), pp.611-619.

5. Shrestha, S. and Chou, K., 2017. A build surface study of Powder-Bed Electron Beam Additive Manufacturing by 3D thermo-fluid simulation and white-light interferometry. International Journal of Machine Tools and Manufacture, 121, pp.3749.

6. Shrestha, S. and Chou, K., 2018. Single track scanning experiment in laser powder bed fusion process. Procedia Manufacturing, 26, pp.857-864.

7. Cheng, B., Shrestha, S. and Chou, K., 2016. Stress and deformation evaluations of scanning strategy effect in selective laser melting. Additive Manufacturing, 12, pp.240-251.

\section{REFERRED CONFERENCE PROCEEDINGS}

1. Shrestha, S., Starr, T. and Chou, K., 2018, November. Porosity Analysis in Metal Additive Manufacturing by Micro-CT. In ASME 2018 International Mechanical Engineering Congress and Exposition (Vol. 52019, p. V002T02A059). American Society of Mechanical Engineers.

2. Shrestha, S., Starr, T. and Chou, K., 2018, November. Individual and Coupled Contributions of Laser Power and Scanning Speed towards Process-Induced Porosity in Selective Laser Melting. In Proceedings of 29th Solid Freeform Fabrication Symposium, Austin, TX, Aug (pp. 13-15).

3. Shrestha, S. and Chou, K., 2018, June. Mesoscopic Simulation Model to Predict Temperature Distribution and Melt Pool Size During Selective Laser Scanning. In ASME 2018 13th International Manufacturing Science and Engineering Conference (Vol. 51357, p. V001T01A043). American Society of Mechanical Engineers.

4. Shrestha, S. and Chou, K., 2018, March. Computational analysis of thermo-fluid dynamics with metallic powder in SLM. In TMS Annual Meeting \& Exhibition (pp. 85-95). Springer, Cham.

5. Shrestha, S. and Chou, Y.K., 2017, June. Process Effect on Part Surface Roughness in Powder-Bed Electron Beam Additive Manufacturing. In ASME 2017 12th International Manufacturing Science and Engineering Conference (Vol. 50732, p. V002T01A017). American Society of Mechanical Engineers.

6. Shrestha, S. and Chou, K., 2017. Mesoscopic multilayer simulation of selective laser melting process. In 28th Annual International Solid Freeform Fabrication (SFF) Symposium - an additive manufacturing conference, Austin August (pp. 7-9).

7. Cheng, B., Shrestha, S. and Chou, Y.K., 2016, June. Stress and deformation evaluations of scanning strategy effect in selective laser melting. In ASME 2016 11th International Manufacturing Science and Engineering Conference. American Society of Mechanical Engineers.

8. Shrestha, S., Cheng, B. and Chou, K., 2016. An investigation into melt pool effective thermal conductivity for thermal modeling of powder-bed electron beam additive 
manufacturing. In International Solid Freeform Fabrication Symposium-An Additive Manufacturing Conference (pp. 208-218).

\section{AWARDS AND HONORS}

- The Manufacturing Engineering Division's Third Place Best Paper Award to "A Numerical Study on the Keyhole Formation During Laser Powder Bed Fusion Process" presented at 2019 Manufacturing Science and Engineering Conference.

- National Science Foundation Travel award to attend Solid Freeform Fabrication conference in 2017, 2018 and 2019

- National Science Foundation Travel award to attend Manufacturing Science and Engineering Conference in 2018.

- Full Scholarship to study Bachelor's in Mechanical Engineering at Pulchowk Campus, Lalitpur, Nepal. 